\title{
GENETIC STUDIES ON A CAVY SPECIES CROSS
}

\author{
BY J. A. DETLEFSEN \\ Assistant Professor of Genetics, University of Illinois
}

WITH A PREFATORY NOTE BY W. E. CASTLE

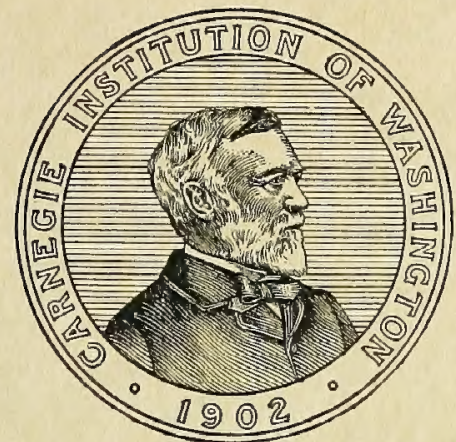

WASHINGTON, D. C.

Published by the Carnggie Institution of Washington 1914 


\section{QH 425.D5 Cornell University Llbrary}

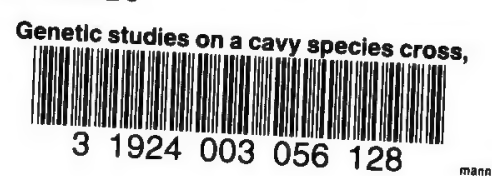




\section{Cornell University Library}

The original of this book is in the Cornell University Library.

There are no known copyright restrictions in the United States on the use of the text.

http://www.archive.org/details/cu31924003056128 
Carnegie Institution of Washington, Publication No. 205

Paper No, 23 of the Station for Experimental Evoldtion at Cold Spring Harbor, New York

From the Laboratory of Genetics, of the Bussey

Institution, Forest Hili, Massachusetts

\author{
Copies of this Book \\ were first issued \\ DEC 311914
}

PRESS OF GIBSON BROTHERS, INC.

WASHINGTON, D. C. 


\section{PREFATORY NOTE BY W. E. CASTLE.}

In July 1903 I received from Mr. Adolph Hempel, of Campinas, Brazil, three wild cavies, a male and two females, of a species supposed at the time to be Cavia aperea, but now referred to Cavia rufescens. The male and one of the females bred in captivity and produced a considerable number of descendants, certain of which (together with the original male) were employed in crosses with ordinary guinea-pigs. The hybrids thus obtained proved completely sterile in the male sex, but the females were entirely fertile. Further propagation of the hybrid race was thus restricted to crossing the female hybrids with males of one of the parent species.

In December 1909 I turned over to my assistant, J. A. Detlefsen, for further study, the stock of hybrid animals, together with the pedigree records and notes of such observations as I had been able to make upon the hybrid race. The present paper will indicate how successful he has been in propagating the hybrid race and what conclusions may be drawn concerning the inheritance of various characters in these hybrids.

The long series of experiments upon which a partial report is here made was rendered possible by a grant from the Carnegie Institution of Washington and by the provision of special facilities on the part of Harvard University. Grateful acknowledgment is made of my obligation to both institutions. 



\section{CONTENTS.}

General Introduction.

Page.

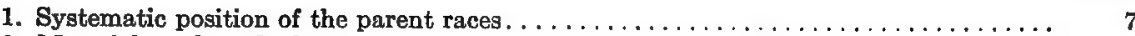

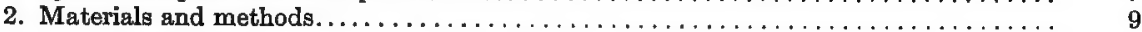

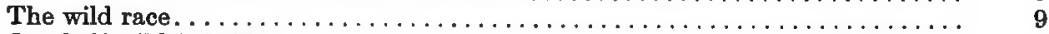

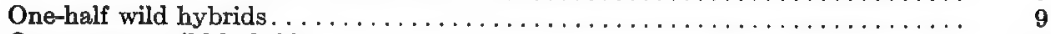

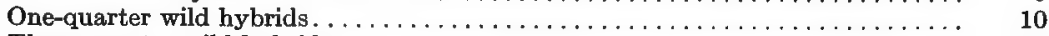

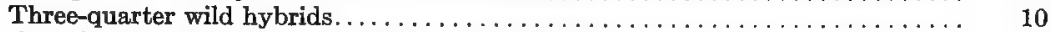

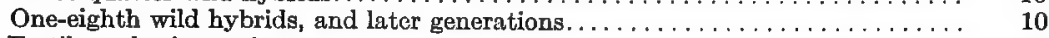

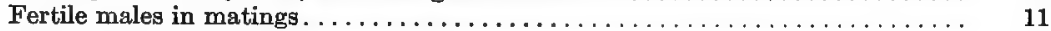

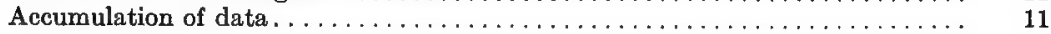

\section{Part I. Color and Coat Characters.}

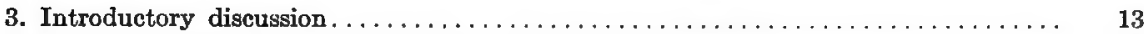

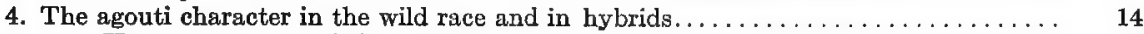

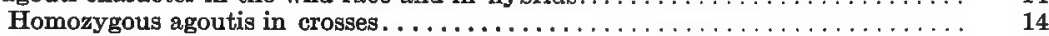

Heterozygous agoutis mated to non-agoutis.................. 17

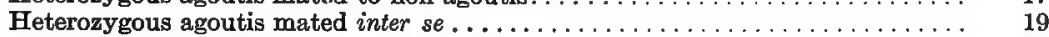

The wild agouti and tame agouti contrasted $\ldots \ldots \ldots \ldots \ldots \ldots \ldots \ldots \ldots \ldots \ldots$

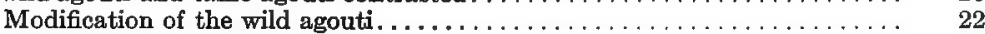

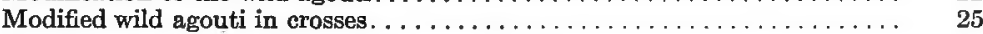

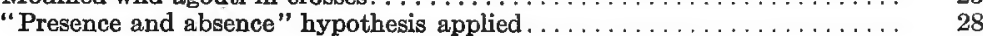

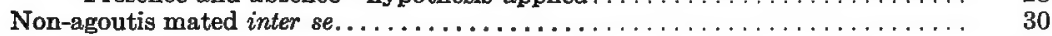

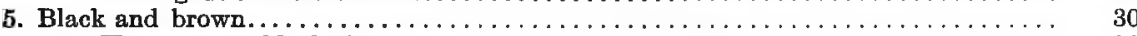

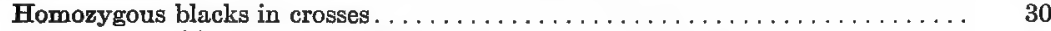

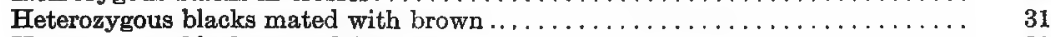

Heterozygous blacks mated inter se........................ 32

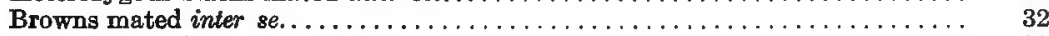

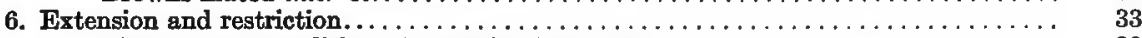

Homozygous condition of extension in crosses . . . . . . . . . . . . . .

Heterozygous condition of extension crossed with restriction . . . . . . . . . . 34

Heterozygotes for extension mated inter se. . . . . . . . . . . . . . . . 35

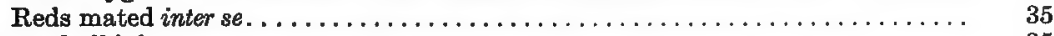

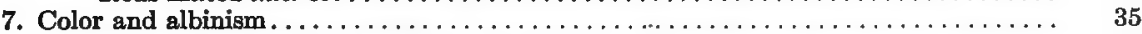

Homozygous condition of the color factor in crosses.............. 35

Heterozygous colored animals in crosses with albinos . . . . . . . . . . . 36

Heterozygous colored animals mated inter se................. 37

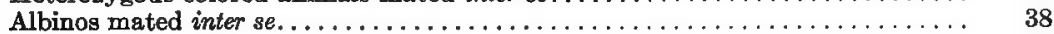

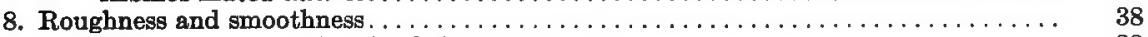

Homozygous rough animals in crosses . . . . . . . . . . . . . 38

Heterozygous rough animals crossed with smooth animals............ 39

Smooth animals mated inter $s e \ldots \ldots \ldots \ldots \ldots \ldots \ldots \ldots \ldots \ldots \ldots \ldots \ldots \ldots \ldots$

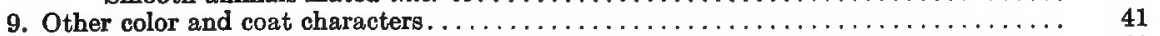

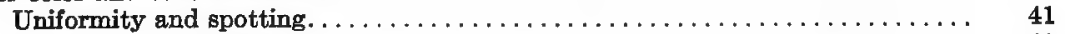

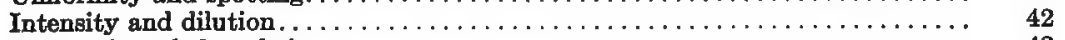

Long hair and short hair........................ 43

10. The fertile hybrid males in color crosses........................ 43

11. General conclusions as to color and coat character. . . . . . . . . . . . 45

Part II. Growth and Morphological Characters.

12. Introductory discussion $\ldots \ldots \ldots \ldots \ldots \ldots \ldots \ldots \ldots \ldots \ldots \ldots \ldots \ldots \ldots \ldots \ldots \ldots \ldots \ldots$

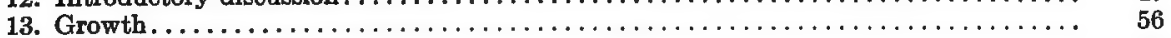

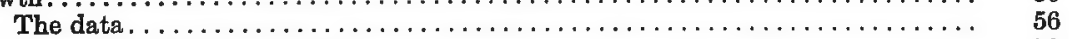

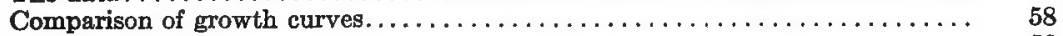

The averages. . . . . . . . . . . . .

The coefficients of variability $\ldots \ldots \ldots \ldots \ldots \ldots \ldots \ldots \ldots \ldots \ldots \ldots \ldots$ 


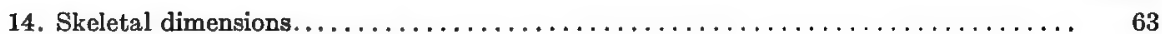

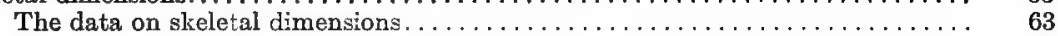

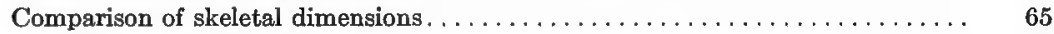

The average dimensions. . . . . . . . . . . . . . . . . . . . 65

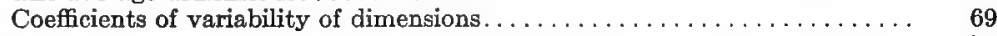

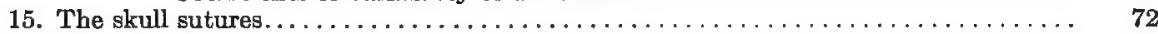

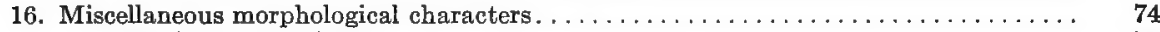

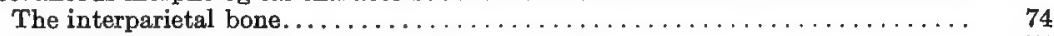

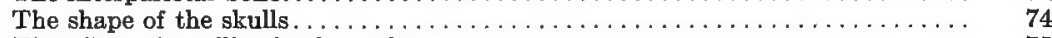

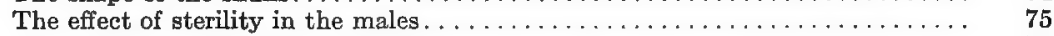

Anomalies occurring in the hybrids. . . . . . . . . . . . . 76

17. General conclusions as to growth and morphological characters $\ldots \ldots \ldots \ldots \ldots \ldots \ldots, 77$

Part III. The Fertility of the Parent Spectes and Hybrids.

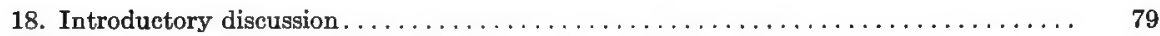

19. The fertility of the male hybrids $\ldots \ldots \ldots \ldots \ldots \ldots \ldots \ldots \ldots \ldots \ldots \ldots \ldots \ldots \ldots, 8 \ldots \ldots$

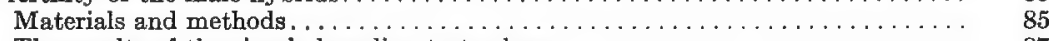

The results of the simple breeding tests alone $\ldots \ldots \ldots \ldots \ldots \ldots \ldots \ldots \ldots$

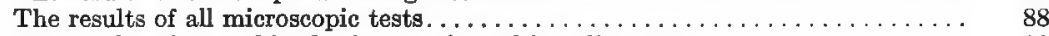

The results of a combined microscopic and breeding test. . . . . . . . . 90

The inheritance of sterility . . . . . . . . . . . . . . . . . . . . . . 92

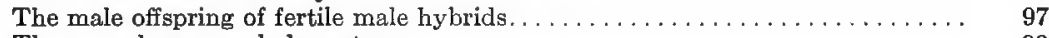

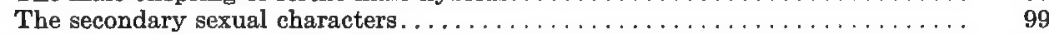

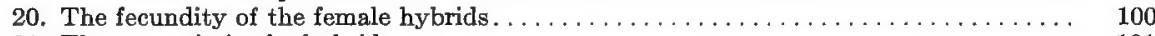

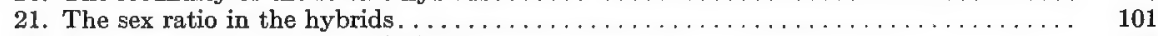

22. Summary and general conclusions. . . . . . . . . . . . . . . . . 103

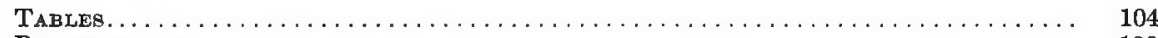

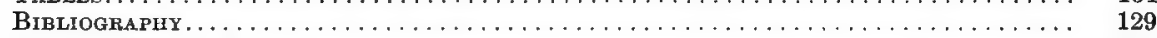

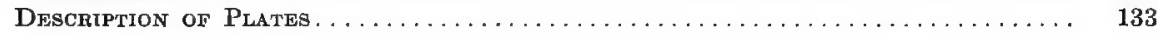




\section{GENETIC STUDIES 0N A CAVY SPECIES CROSS.}

\section{GENERAL INTRODUCTION.}

The genetic studies herewith presented were made possible for the author, by the reception of the foundation stock, in December 1909, from Dr. W. E. Castle. The first crosses had been made in 1903, and about 200 of the wild and intense wild-blooded hybrid animals had been born when the stock was received. The birth records, the weights, and such skeletons as had been saved, as well as the living hybrids, were made available to the author, who here expresses his gratitude for the privilege of using this material and for generous assistance, which was never withheld. He also wishes to acknowledge the valuable aid of Mr. Elmer Roberts, in the preparation of the manuscript.

Most of the manuscript was written and most of the data were analyzed at the College of Agriculture of the University of Illinois, to which the author is deeply indebted for liberal use of time and facilities.

\section{THE SYSTEMATIC POSITION OF THE PARENT RACES.}

This paper is based on a study of the wild Brazilian guinea-pig, (Cavia rufescens Lund), the common domestic guinea-pig (Cavia porcellus Linn.), hybrids between these, and subsequent progeny obtained in the next eight generations by various matings. About 1;800 animals, wild or hybrid, enter in one way or another into experiments on color, growth, size, and fertility. Besides these, approximately 600 guineapigs, living under the same conditions in collateral experiments, serve as a basis for necessary comparisons.

That the hybrids are the result of a species cross rather than a variety cross can hardly be doubted, since the $\frac{1}{2}$ wild and $\frac{1}{4}$ wild males are entirely sterile. In order to meet any doubt or criticism at the outset, I may briefly give my reasons for assigning the parent stocks to such diverse and distantly related species. In the summer of 1903 Dr. W. E. Castle received one wild male and two wild females from Mr. Adolph Hempel, Campinas, São Paulo, Brazil. These and their progeny were kept for some time at the Harvard Zoological Laboratory, and were removed later to the Laboratory of Genetics, Bussey Institution, Harvard University. In the summer of 1911, three years after the last animal of pure wild pedigree had died, we again received from Mr. Hempel one wild male and one wild female. At first it was thought 
that these wild cavies belonged to the commonly described Cavia aperea Erxleben, but a more careful investigation showed later that they belonged to the less well-known Cavia rufescens Lund (Lund 1841, Waterhouse 1848, Thomas 1901). This cavy is considerably smaller than Cavia aperea or Cavia porcellus, both in total size and in the individual bone measurements. Thomas asserts that Cavia rufescens never reaches the size of Cavia aperea. The color is agouti or "ticked," as in most wild rodents, but somewhat darker than the agouti of Cavia porcellus, because more black shows in the individual hairs and less yellow on their subapical bands. The belly varies from a light yellow to a slightly ticked condition. The systematists lay great stress on the formation of the last upper molar, in which a deep, narrow indentation on the outer surface almost separates the small third lobe from the body of the tooth. Lund describes his specimen from Minas Geraes, Brazil. In all essential points the wild animals in this experiment agree with the descriptions, plates, and general locality given by the above-mentioned authors.

A report of the experimental work does not necessitate an argument on the number of differential characters which would infallibly place two types in those more or less arbitrary categories- "species." It is sufficient for the purposes of this problem to find that the wild cavies used belong to a species more distantly related to the tame guinea-pig than are Cavia aperea or Cavia cutleri, according to the methods of most taxonomists. The taxonomists differ much among themselves. For instance, Waterhouse held that Cavia porcellus, Cavia aperea, and Cavia cutleri might all be placed in the same species. He found forms bridging typical differences. Darwin (1876) held that Cavia aperea was not the ancestor of the guinea-pig, basing his views on the fact that a distinct genus of lice infested each form. As far as his evidence goes, it might be considered decisive, for entomologists have reported that closely related mammals are infested by closely related lice (Osborn 1908). Giebel (1855) placed a number of cavy forms in the species aperea, and held that Cavia rufescens was only a variety of the larger Cavia aperea. Nehring (1889) considered Cavia cutleri to be the direct ancestor of our tame guinea-pig, being inclined to such a view on both historical and morphological grounds. He later showed (Nehring 1893, 1894) that Cavia aperea may be reciprocally crossed with the guineapig and give perfectly fertile offspring-fertile inter se or when mated back to either parent. Thomas (1901) is in doubt as to which of the two wild forms, Cavia aperea or Cavia rufescens, is the real ancestor of the guinea-pig. It would appear, from a comparison of Nehring's experiments and the experiments described in this paper, that Cavia aperea must be more nearly related to the guinea-pig than Cavia rufescens is, for the latter gives sterile male offspring in a cross with the tame guinea-pig, whereas Cavia aperea does not. 
It would be equally difficult to formulate any rule by which we could determine how great must be the difference in color, shape, size, tooth formation, and the like, between genuine "species," but for our present purposes this, fortunately, is unnecessary.

The reasons for considering the wild stock used in these experiments to be specifically distinct from the guinea-pig are as follows:

(1) The skull characters, size, and color of our wild stock undoubtedly place it in the species rufescens. I am indebted to Dr. G. M. Allen for a corroboration of this classification.

(2) Hybrids between our wild stock and the guinea-pig are sterile in the male sex, regularly through two blood dilutions and in many cases through more blood dilutions.

The other parent species, the common domestic guinea-pig, Cavia porcellus (also called Cavia cobaya), is too well known to require identification or description. The peculiarities of the stock used in these experiments, if there be such, are described in the detailed discussion of their inheritance. The ancestors of the guinea-pigs, in these experiments, were obtained by purchase from dealers and fanciers, but the animals which were used were of known zygotic color formulæ, size variability, and fertility.

\section{MATERIALS AND METHODS.}

THE WILD RACE.

The original wild $\sigma^{\prime} 1$ was mated to wild $q \% 2$ and 3 , to increase the stock. (See fig. 1.) He was likewise mated to his daughters, as were his sons, $\sigma^{2} 24$ and $0^{7} 33$, and his grandson or55. The young of $\$ 2$ died prematurely, and so do not figure in any of the later crosses; hence all the wild stock came from two original parents, $\sigma^{r} 1$ and $q 3$. The pure wild line eventually died out, for, even with the greatest care and experience in handling domestic cavies, it was not possible to carry the wild stock more than 5 years in captivity. The animals were prone to fight. Only one female could be penned with one male at the same time. The total number born in captivity was 46 , but of these only 4 females and 3 males reached sexual maturity. Our experience does not agree with that of Nehring (1894), who realized little difficulty with Cavia aperea in captivity. This fact again distinguishes the two stocks and experiments. The two wild cavies received in 1911 have not bred up to the time of writing.

\section{ONE-HALF WILD HYBRIDS.}

The original wild male, $\sigma^{7} 1$, and his sons, $\sigma^{7} 24$ and $\sigma^{7} 33$, and his grandson, $\sigma^{7} 55$, were used to obtain hybrids between the pure wild stock and the tame guinea-pig. (See fig. 2.) The reciprocal cross (tame male $X$ wild female) was not obtained or even attempted, for it was feared that such small females might die in pregnancy when 
impregnated by the larger-sized guinea-pig male. The matings were obtained with much difficulty, for the wild sire at first harassed and bit the tame females almost beyond recognition; but, by keeping him in solitary confinement for some time, and then placing him with a female which had just given birth to young, copulation was successfully brought about. The young appeared in due time (63 to 67 days) and in the usual guinea-pig number, showing that such wild males, producing an abundance of sperm, are wholly fertile with tame females. Our stock of tame females used as the mothers of the hybrids consisted of large healthy animals of known color varieties (except the dams in two cases of young not used in further experiments). The offspring were all agouti-colored like the wild father; 39 such $\frac{1}{2}$ wild offspring were obtained, but of these only 10 females were successfully used for breeding purposes. The males were all sterile.

I have used the terms $\frac{1}{2}$ wild, $\frac{1}{4}$ wild, $\frac{1}{8}$ wild, $\frac{1}{16}$ wild, etc., but wish to state here that these terms are used only for convenience, without implying blending inheritance. They simply denote the generation to which a hybrid belongs.

\section{ONE-QUARTER WILD HYBRIDS.}

Since the $\frac{1}{2}$ wild males were sterile, the $\frac{1}{2}$ wild females were mated to both parent stocks. When mated to the guinea-pig they produced $\frac{1}{4}$ wild rufescens hybrids; but when mated to the wild Cavia rufescens they produced $\frac{3}{4}$ wild rufescens hybrids. Of the $\frac{1}{4}$ wild young 83 were obtained, sired by pedigreed male guinea-pigs. In this blood the males were again sterile; therefore the females were mated back to guineapig males. The numbers of sexually mature females increased with each generation; hence there was no difficulty in procuring sufficiently large numbers of the more dilute-blooded hybrid animals.

THREE-QUARTERS WILD HYBRIDS.

Only one wild male (o $0^{74}$ ) and one $\frac{1}{2}$ wild female ( 950 ) were used for this part of the experiment, and they produced four young, of which two, a male and a female, reached maturity but proved to be sterile. (See fig. 3.) The wild males died out soon after this, and effectually put an end to this class of matings.

\section{ONE-EIGHTH WILD HYBRIDS, AND LATER GENERATIONS.}

Proceeding in the same manner used to obtain the previous generations, the females of one blood were continually mated back to guineapig males to produce animals of the next blood-dilution. Thus, from our $\frac{1}{4}$ wild females we obtained $\frac{1}{8}$ wild, and from the $\frac{1}{8}$ wild females we obtained $\frac{1}{16}$ wild. Up to the time of writing, the blood has been reduced to $\frac{1}{25}$ wild, with $\frac{1}{512}$ wild young in utero; $i$. e., the $\mathrm{F}_{9}$ generation. Naturally most of the animals now living are not so far removed 
as this from the original stock. At present most of our pens contain $\frac{1}{32}$ wild hybrids.

The numbers of hybrids obtained up to October 1911 were as follows: $\frac{1}{2}$ wild, $39 ; \frac{3}{4}$ wild, $4 ; \frac{1}{4}$ wild, $83 ; \frac{1}{8}$ wild, $217 ; \frac{1}{16}$ wild, $312 ; \frac{1}{32}$ wild, 344 ; $\frac{1}{64}$ wild, $122 ; \frac{1}{128}$ wild, 37 ; $\frac{1}{256}$ wild, 2 ; total, 1,160 . Since that time 600 more hybrids have been born.

Unfortunately, for comparisons, mammalian species crosses are not common. When they have been made the number of offspring has been small, thus affording small basis for generalization. The most reliable data are drawn from species crosses among the ungulates, but ungulates are not adapted to laboratory experiments in large numbers. Species crosses are unknown among the Monotremata, Edentata, Insectivora, Chiroptera, Sirenia, Proboscidea, and Hyracoidea (Przibram 1910). The species crosses among ungulates, like horse and ass, or cow and bison, involve the question of sterility and fertility. The similar sterility in the cross of the wild and tame guinea-pig affords excellent material for comparison with these larger economic forms.

FERTILE MALES IN MATINGS.

The most interesting part of the whole problem is the origin of fertile hybrid males and crosses of such males with females of the different blood-dilutions and with guinea-pig females. Seven fertile males appeared among our $\frac{1}{8}$ wild hybrids. The number increased rapidly in the $\frac{1}{16}$ wild, $\frac{1}{32}$ wild, and later generations. The importance of these males is apparent; for it gives opportunity to study sterility and fertility, and to test whether any segregation of characters in the direction of Cavia rufescens is possible. Previously, any segregation possible was in the direction of the guinea-pig, Cavia porcellus. The fact is realized that a large number of characters is involved, and it will therefore require the observation of many individuals before we can reasonably expect to observe complete segregation of either the guinea-pig or the rufescens characters as a group. Fortunately many of the characters are so unmistakable and definite as to allow of no doubt or uncertainty in their case. The detailed result of the matings of the fertile male hybrids is given in Part III. The young from such matings have not reached maturity and consequently their bone measurements and growth curves can not be given at this time.

\section{ACCUMULATION OF DATA.}

It has been stated that a number of differential characters mark the wild guinea-pig in distinction from the tame. Records of the expression of these characters and new characters which appeared have been made.

Color.-Cavia rufescens is not known to occur in any color, except agouti of a rather distinct and specific type. Color records of each hybrid were made at birth. 
Growth.-The wild species was observed to grow more slowly than the tame guinea-pig. Hence weights of the wild, the hybrids, and the tame were taken at frequent intervals and recorded. The animals were weighed at birth, or shortly after, and then each week until the nature of the individual growth curve was established. After that they were weighed at less frequent intervals until they died or were killed. This method also afforded an opportunity to keep strict watch on the health of each animal, for a sharp, unexpected drop in weight indicated sickness, fighting, or some other disturbing cause. Sexually mature females were weighed immediately after parturition, in order to eliminate the error due to a varying number of fetuses.

Skeletal dimensions. - Just as the wild animal is smaller in total size, so its individual bones were observed to be shorter and more slender than those of the tame. The skull, lower jaw, right scapula, right foreleg, and right hind-leg of such adults as died were saved for further observations. Whenever a hybrid reached maturity and could no longer be used for other purposes, it was killed and the bones similarly saved. A careful examination of the growth curve and the bone sutures showed that guinea-pigs and hybrids are of full adult size when 15 months old. Measurements, of which a detailed account is given later, were made and tabulated.

Fertility. - The fertility of the wild, hybrid, and tame females was not uniform. Records of the size of each litter were kept, from which averages could be calculated. The wild males were fertile in captivity, but their $\frac{1}{2}$ wild hybrid sons and their $\frac{1}{4}$ wild grandsons were sterile. The problem immediately suggested itself: how great must be the blood dilution, or for how many generations must the hybrid females be crossed back to the guinea-pig, before producing fertile males? The numbers of males to be tested increased to such an extent that facilities were lacking to test their fertility by mating them to females. Furthermore, it is well known that a male may be potentially fertile, but fail to show it because of some physiological state, such as extreme emaciation from sickness, or through the sluggishness of obesity. Another method was devised. It was observed from many cases that the breeding test was negative whenever a male lacked spermatozoa in the epididymis or when these spermatozoa were few, degenerate, or nonmotile. On the other hand it was found that fertile males invariably have many motile spermatozoa in the epididymis. Examination of the sperm content of the epididymis therefore affords a clear index of fertility. The examination is readily accomplished by placing a drop of the contents of the epididymis in normal salt solution at a bodily temperature and examining it under the microscope. An operation of this sort performed on one side of the body only does not preclude subsequent breeding of the animal operated upon. 


\section{PART I. COLOR AND COAT CHARACTERS.}

\section{INTRODUCTORY DISCUSSION.}

Instances of alternative or Mendelian inheritance have been rapidly accumulating since the rediscovery of Mendel's law in 1900, but most of the cases known among mammals are based on relatively simple and easily executed crosses, namely, crossing varieties of a species. Hence the criticism has been offered that this form of inheritance does not occur in species crosses or in nature. It has been maintained that Mendelian phenomena are the result of laboratory methods, in which we deal with man's domestic varieties. No contention is offered that this or any other wild cavy mates with the guinea-pig in nature. We have no evidence for or against such an hypothesis. In fact, it is more probable that such crosses do not occur, for the repulsion which one species of mammal usually shows to mating with another was evident even in this experiment. When, however, a species cross is actually made, whether it is in the laboratory or elsewhere, the data accruing from the experiment may be legitimately offered to bear on the mode of color inheritance in a species cross.

The papers of Castle $(1905,1905 a, 1907,1907 a, 1908,1909)$ and Sollas (1909) deal with the subject of color inheritance in guinea-pigs in a summary manner, and so much has been written upon this subject in other forms that I should feel most apologetic in offering more data upon alternative inheritance of color in plants or animals were it not for the fact that my observations cover a very definite category of cases which have received little attention up to the present time, and which may be of some general interest to students of heredity because of the nature of the cross which gave rise to them.

The symbols used to designate the color and coat factors are, briefly, as follows:

C, a factor necessary to the production of color in animals. Albinos lack this factor; the allelomorphic condition is represented by c.

A, a factor restricting black or brown in the individual hairs, producing the ticked or agouti type of coloration. This factor may restrict differently in different parts of the coat. Black and brown are restricted in the yellow subapical band on the dorsal surface. They may be completely restricted on the belly, giving yellow belly, as in the domestic guinea-pig; or they may be partially restricted, and so allow a ticked or barred appearance on the belly as well as on the back. The latter is the condition in some wild Cavia rufescens and some hybrids. The allelomorphic condition is designated by a. 
$\mathrm{B}$, a factor for black. Black is usually considered as the most complete oxidation product of the yellow-brown-black series. Animals lacking this factor to produce black are brown, or can transmit only brown. The latter condition is indicated by $b$.

$\mathrm{E}$, a factor for the extended condition of black and brown pigmentation, in distinction from the restricted condition. This factor produces self-colored black or brown animals, whereas its absence, designated by e, is characteristic of the black-eyed or brown-eyed red or yellow coat.

$\mathrm{Rf}$, a factor for the rough or rosetted coat character. Smooth-coated animals lack this factor, and the condition is represented by $\mathrm{rf}$.

Each color table deals with a single allelomorphic pair, thus keeping the ratios as simple as possible. A number of years ago it was necessary to explain the various kinds of chance ratios, but such discussion may now be advantageously omitted. Likewise it has been shown that the ratios obtained by dealing with two, three, or more pairs of allelomorphs without coupling are the squares, cubes, or higher powers of the simple $3: 1$ ratio. Hence, it is obvious that the more complex ratios may be obtained from the simple and we need not deal with all the color characters of each animal at one time, but just deal with a single character and its allelomorph in each case. The tables deal with zygotic constitution rather than somatic appearance; for instance, an albino may transmit agouti, and therefore be entered in a table in which all the animals entered transmit this factor, irrespective of the somatic colors, or lack of color.

\section{THE AGOUTI CHARACTER IN THE WILD RACE AND IN HYBRIDS.}

HOMOZYGOUS AGOUTIS IN CROSSES.

Agouti, the factor which restricts black or brown from the sub-apical portion of the hair and gives a barred appearance, is characteristic of Cavia rufescens. The character is common to all wild rodents. A number of investigations on rats, mice, and rabbits (Cuénot 1903, 1904, 1911; Castle 1905, 1905a, 1907, 1907a, 1908, 1909; Hurst 1905; Sollas 1909; Morgan 1911) give sufficient evidence that it acts as a unit character, dominant to the non-agouti condition, and segregating in the $\mathrm{F}_{2}$ generation, according to Mendel's law.

The agouti of Cavia rufescens is of somewhat different appearance from that of Cavia porcellus or Cavia cutleri. It is darker than either, showing a narrower yellow subapical band and more black. There is some variation in this character in the wild rufescens, which accounts for slight differences in systematic descriptions. The belly-hairs of Cavia rufescens vary from yellow to slightly ticked, but in Cavia porcellus the variation is from complete yellow to yellow with a small amount of black at the base. In both species there is a constant relation 
between dorsal and ventral pigmentation; for the darker the dorsal surface, the darker is the ventral surface, and in any one animal of either species the dorsal surface is always darker than the ventral.

Two facts may here be emphasized:

(1) The agouti of the wild rufescens has less power to exclude black and brown from the hair than has the agouti of the tame.

(2) Agouti, from whatever source derived, produces a more striking effect on the belly than on the back, restricting black or brown more completely in the former region. It is one and the same agouti factor, but it causes a different reaction in these two parts of the coat. Breeding many agoutis has shown that there are not two factors, one for restriction of black on the ventral side and one for the restriction of black on the back. If this were true, the two factors could be dissociated and transmitted independently, but this has never been accomplished. It may be objected that, even with this evidence, we can not be sure that two or more factors do not exist in complete coupling. The objection, in a measure, answers the argument, for if the coupling of factors is complete, we can only deal with them as one unit character.

The surmise that the wild race would be found homozygous in agouti proved true (see table 1 ). The original wild male was father of 27 young, all agouti, like himself, while his sons and grandson sired 19 other agoutis. Had either one of the original parents, $\sigma^{\top} 1$ or $q 3$, been heterozygous, it would have been possible to extract recessive nonagouti individuals from the matings of their offspring inter se, for half of their offspring would likewise have been heterozygous. The conviction that the wild race must be homozygous in agouti is furthermore strengthened beyond a reasonable doubt by the matings of 4 wild males with 10 different non-agouti guinea-pig females (table 2); 37 agouti young were thus produced. If the wild parent in any of these matings had been heterozygous, these matings must surely have produced some non-agouti offspring, but such was not the case. Our point is therefore well established by these 83 offspring. A second, point, in a measure dependent on the first, may be stated thus: the wild agouti character dominates its absence, even though the absent condition is presented by the tame female parent. ${ }^{1}$ In both particulars the results agree with similar matings among guinea-pigs. Such was Nehring's experience, also, with Cavia aperea; for, though he did not understand alternative inheritance at the time of his experiments in 1893, he nevertheless gives data which show conclusively that Cavia aperea, a different wild Brazilian species, is likewise homozygous in agouti and dominant to its absence in a mating with Cavia porcellus. It is rather surprising that no one has studied Nehring's data and referred to them or pre-

${ }^{1}$ Castle (1905) has reported on the dominance of the wild agouti when mated to non-agouti. The wild stock at that time was supposed to be the common Brazilian Cavia aperea. The fact of dominance reported was correct; the error of classification is corrected in this paper. 
sented them as evidence of Mendelism in a species cross, when the criticism on alternative inheritance in species crosses was first made. Possibly it was the lack of numbers in his experiments, but surely, as far as they go, the results are quite conclusive on this, as well as some other points.

All $\frac{1}{2}$ wild hybrids recorded in table 2 were heterozygous in agouti, for they were the result of matings between wild agouti males and non-agouti females. The agouti which they bore came from one definite source, the wild strain. Tables 1 to 12 deal with both tame and wild agouti as one. This method of procedure is followed because both wild and tame agouti have many common characteristics. The discussion of their differences is reserved for tables 13 to 15 .

It has been proven that agouti obtained from the wild is dominant over the non-agouti condition in the tame. Therefore a number of matings were made to investigate the reciprocal cross, in which tame agouti guinea-pig males were mated to wild hybrid females. Two homozygous agouti males ( $\sigma^{\top} 1961$ and $\sigma^{7} 2157$ ) were mated to 10 different $\frac{1}{8}$ wild females (table 3 ); 3 of these females were heterozygous in wild agouti, and the rest were non-agouti animals; the 27 young obtained were all agouti, like the father. These young should be of two zygotic classes; those produced by the 3 agouti females should half of them be homozygous and the remainder heterozygous agouti animals, whereas all the young from the 7 non-agouti females should be heterozygous. Both zygotic classes were produced; for in testing the offspring of the 3 heterozygous females, one female (580) was found to be heterozygous, and one female and one fertile male ( 9485 and $\sigma^{7} 506$ ) were found to be homozygous. But the offspring of the 7 non-agouti females used were invariably heterozygous. The result of these matings shows that agouti obtained from either wild or tame is dominant to non-agouti, whether this latter condition is derived from tame females (table 2) or from hybrids (table 3 ).

The matings indicated in table 4 corroborate this view. In this experiment 5 different $\frac{1}{16}$ wild hybrids were used. The hybrids were the result of matings calculated to produce homozygous agouti by crossing females of the wild agouti type with males of the tame agouti type. These 5 agouti hybrids showed their homozygous character by producing 21 offspring, all agouti. Their gametes evidently carry agouti in all cases, although this agouti was derived from two very different sources, the wild and the tame. When such gametes are formed they are presumably of two types, one bearing wild agouti and one bearing tame agouti; and when they meet gametes without agouti, the zygote formed produces an agouti animal, the agouti being theoretically in one case like the wild and in the other like the tame. The numbers are small, but quite conclusive; for not only were all the offspring agouti, but among them occurred agouti individuals of two 
different sorts, one sort resembling the agouti of $C$. rufescens, the other that of the guinea-pig. If we designate the tame agouti as $\mathrm{A}$ and the wild agouti as $\mathrm{A}^{\prime}$, then these five parents had a zygotic formula of $\mathrm{AA}^{\prime}$. It is evident, then, that they must have produced certain gametes which bore $\mathrm{A}$, the powerful tame agouti factor, and others which bore $\mathrm{A}^{\prime}$, the weak wild agouti factor. The young accordingly were of two sorts, wild and tame agouti. This subject will receive consideration in a later part of this paper. For the present, all kinds of agouti will be considered as one, irrespective of their source.

Summary.-The wild Cavia rufescens is homozygous in agouti. This condition is epistatic to the non-agouti condition of the tame guineapig. The agouti of the tame is likewise epistatic to the non-agouti condition of the hybrids. Hybrids may be produced which are homozygous in agouti. In table 5 the summary of tables 2,3 , and 4 shows that 85 agouti offspring were produced from matings of pure agouti animals. Therefore the agouti factor is epistatic, whether found in the wild, the tame, or the hybrid. This agrees with Nehring's results on Cavia aperea, though his interpretation was different. To make data plain and not suppress any facts, it should be stated that a few albinos enter into some of the tables. Such albinos, we know, carry all color factors in the same proportions as their colored brothers and sisters, with the exception of the basic color factor itself. It may therefore be understood that albinos have been omitted from the tables, unless a thorough breeding test has demonstrated to what color class each albino belongs, in which case it has been included in the corresponding colored class.

\section{HETEROZYGOUS AGOUTIS MATED TO NON-AGOUTIS.}

All the $\frac{1}{2}$ wild hybrids derived from the cross (table 2) of a Cavia rufescens male with female guinea-pigs were supposedly heterozygous in agouti; 9 female $\frac{1}{2}$ wild hybrids were mated with male guinea-pigs. The sterility of the male hybrids prevented a breeding test in their case. The female $\frac{1}{2}$ wild all bore the agouti coat and had received the agouti factor from the wild parent. A priori, they should have been heterozygous in this factor, having received it from one parent only. Such they proved themselves to be in their matings with the non-agouti guinea-pig males. They gave offspring of two sorts, agouti and nonagouti (in this case all were black) in approximately equal numbers; 83 such offspring (table 6) were obtained, of which 47 were agouti (also heterozygous) and 36 were non-agouti. To strengthen the case, it may be pointed out that each female $\frac{1}{2}$ wild should prove her heterozygous condition by giving both sorts of young, provided the numbers are large enough; 7 of the 9 females gave both sorts of young. One female ( 975 ) gave 4 agoutis only, and another female ( $\$ 72$ ) gave 4 nonagoutis. Presumably these last two females would have yielded both 
classes of young had they been more prolific. The law of probable error would account for the occasional occurrence of these ratios of $0: 4$ and $4: 0$, where we expect equality as an average result.

It is, therefore, clear that when a wild species of cavy known to be homozygous in agouti is mated with a tame race lacking agouti, and hybrid females are thus produced, these are heterozygous in the agouti factor.

Let us follow the $\frac{1}{4}$ wild agouti offspring of the heterozygous $\frac{1}{2}$ wild females. Since they were produced by matings in which only one parent (the $\frac{1}{2}$ wild) carried agouti, they too should be heterozygous; 20 females of the 47 agouti $\frac{1}{4}$ wild individuals were mated to non-agouti guinea-pig males (table 7). Just as in the matings of table 6, each female should in this case produce both agouti and non-agouti young. Females 95, 97, and 98 produced young of only one kind as far as we know, but since the total young of these 3 females is only 4, we may legitimately neglect them. The total number of offspring of all the females in this experiment (table 7) was 55 agouti and 59 non-agouti, a close approximation to the expected equality.

It is interesting to note that, whereas the $\frac{1}{2}$ wild females gave a slight preponderance of agouti young, the $\frac{1}{4}$ wild agouti females gave the reverse. Adding the matings of tables 6 and 7 , we see that our intense wild-blooded hybrids acted just as the guinea-pig does in matings of this description, and produced an approximate equality of agouti and non-agouti young, in this case 102 agouti to 95 non-agouti. The most probable expectation is either 98 or 99 of either sort.

We have traced (in tables $8,9,10$ ) the matings of all the rest of our heterozygous agouti females with non-agouti males. Since, in the intense wild-blooded hybrids, the color inheritance for agouti has been shown to be the same as that described by Castle (1905) and Sollas (1909) in the guinea-pig, we had no reason to expect our dilute-blooded hybrids to behave differently, for they surely are still more like guineapigs than the earlier generations of hybrids. In table 8 are summarized the matings of heterozygous $\frac{1}{8}$ wild females with recessive guinea-pig males and in the lower division of the table matings reciprocal to those just described. Since the reciprocal matings gave like results they may be combined. The offspring, all told, are 50 agouti and 37 non-agouti. In tables 9 and 10 .are summarized matings in which the females alone bore agouti. They evidently produced gametes of two sorts in equal numbers, those bearing agouti and those without it.

Tables 6 to 10 deal with similar matings, namely, the heterozygous agouti mated to non-agouti, in the different blood dilutions. The summary of these experiments constitutes table 11. It is noteworthy that some of these agouti hybrids received their agouti character from the original wild parent and some others (after the $\frac{1}{8}$ wild of table 8) received the agouti from the tame. The two are distinguishable. 
The tables show that the wild agouti has been kept in a heterozygous condition up to the $\frac{1}{82}$ wild females. Matings made since these tables were constructed prove the same up through the $\frac{1}{1 \frac{1}{28}}$ wild, $i$. e., for seven generations. In other words, one dose of agouti was received from a wild race, and this one dose was handed on for seven generations; and each female that received it passed it on to one-half of her offspring in the next more dilute generation. Also, one dose of agouti derived from tame guinea-pigs was given to some $\frac{1}{16}$ wild hybrids, and this was similarly inherited for three generations. In all these cases, agouti may be said to act as a unit character, just as in the well-known tame crosses.

Summarizing all the matings of all generations of hybrids (table 11), in which one parent is heterozygous in agouti and the other is recessive, such matings have produced 226 agoutis and 214 non-agoutis. The most probable expectation is 220 of each sort. A departure of 6 individuals is explicable by the law of chance. ${ }^{1}$

\section{HETEROZYGOUS AGOUTIS MATED INTER SE.}

The matings of female hybrids, heterozygous in agouti, to male guinea-pigs, likewise heterozygous in agouti, are of very limited number, but more are in progress at the present time. Eight female hybrids, known to be heterozygous, were mated to 5 different male guinea-pigs, also heterozygous. The results of these 8 matings (table 12) are 36 offspring, of which 32 are agouti and 4 non-agouti. The most probable expectation is 27 agoutis to 9 non-agoutis. In these matings, $\sigma^{7} 1436$, $\sigma^{7} 2196$, and $\sigma^{7} 2002$ did not produce any recessives, yet table 8 shows that $o^{7} 2196$ and o'2002 were heterozygous. Male 1436 is known to be heterozygous from pedigree, so that his 4 agouti young (table 12) do not indicate any error. Male 1917 (table 12) produced 9 agoutis and only 1 non-agouti. The ratio $32: 4$ shows a considerable excess of agoutis over the usual $3: 1$. Such deviations are usually explained by the Law of Error, according to which any ratio might be obtained in place of a $3: 1$; but the wide departures from such a ratio must occur with minimum frequency. Possibly the deviations observed in this case are due to chance.

In mating heterozygotes inter se we expect two visible classes, but three actual zygotic classes. One-third of the agouti individuals should breed true; two-thirds should be heterozygous; the recessives should breed true. To test the validity of the ratio, the breeding records of the agouti animals produced by the experiment of table 12 have been studied. It was possible to mate 12 agouti females and 1 fertile agouti male to non-agouti guinea-pigs. The rest of the 32 agouti animals

\footnotetext{
ISince these records were made, 103 young have been born in crosses similar to those above. of these young, 46 were agoutis and 57 were non-agoutis. Adding these to those previously obtained, we have a ratio of 272 agoutis to 271 non-agoutis-actually the most probable expectation.
} 
either died or were sterile males; 8 of the 13 animals tested have proved to be heterozygous, 3 homozygous, and 2 are questionable, for the last produced only agouti young, but in such small numbers that no conclusions can be drawn in regard to their zygotic formula. It is apparent that both the expected classes of agouti individuals were produced, and that the heterozygotes (8) occur approximately twice as frequently as the homozygotes ( 3 to 5 ). These homozygous animals are interesting particularly because the agouti came from two sources, the wild and the tame, and they produced agouti young of two sorts. It seems paradoxical to assert that a homozygous agouti animal produces two sorts of agouti, yet, as we have already observed, the agouti of $C$. rufescens is distinguishable from that of $C$. porcellus.

There is a sharp distinction between a factor and its allelomorph. No matter how much variation there may be in the tame agouti pattern, it always segregates clearly from its absence. The same has been shown for the wild agouti in tables 6 to 10 . There is a certain amount of variability to all unit characters. This is especially true of the wild agouti pattern in a heterozygous condition in hybrid animals. Where the wild agouti pattern has been so modified in the hybrid animals that it can be distinctly discriminated from the tame, it offers splendid material for a cross with tame agouti. Although the wild has been described as somewhat darker than the tame agouti, hybrids arose which were nearly black, so weak was the wild agouti factor (see figs. 4 to 9 ). Without further preliminaries, the variability of the wild agouti and its action in crosses with the tame may be appropriately discussed.

\section{THE WILD AGOUTI AND TAME AGOUTI CONTRASTED.}

In the preceding discussion all agouti individuals have been classed together, irrespective of the differences which have been indicated as distinguishing wild from tame animals. Such is the usual method of procedure in genetic studies. For instance, in crosses of English-patterned rabbits, bearing a dominant restricting factor, with self-colored rabbits, the English pattern is held to act as a unit. The differences between various animals, possessing the same unit character, are explained by postulating either variability in this one unit character or a number of similar or dissimilar genes for this one character, or other modifying unit characters, such as intensity, dilution, and the like.

Black, in crosses, is dealt with in much the same way, and differences, easily discernible or seen with difficulty in different individuals, are similarly explained. A clearer example of this is shown in the crosses of hooded and self-patterned rats. The hooded pattern shows a very wide range of variability, yet any hooded pattern acts as a unit in crosses with self. Pure genotypical races as regards color in animals have not been isolated. Even agouti itself, in variety crosses, has been 
treated as a unit; yet, in the guinea-pig, differences in the agouti factor can be seen. Recently Morgan (1911) has reported on a cross between gray-bellied agouti mice and light-bellied agouti mice, and although the numbers given are small, it is quite clear that each form acts as a unit, and that the gray-bellied agouti is recessive. Although Morgan does not state it, it would appear that the difference between gray-bellied agoutis and light-bellied agoutis is not a difference in separable bellyticking factors. The difference is probably a difference between two kinds of agouti, in which the peculiarity of one agouti is a weakened restricting power and the consequent appearance of black on belly hairs, whereas the other agouti is a more powerful restrictor and therefore gives yellow or light belly, without the usual black in the belly hair.

It has been found expedient to treat all kinds of agouti as one, whether found in the wild, tame, or hybrids. This treatment of the ticking factor has been adhered to, because all forms of agouti have some qualities in common, and whatever the agents may be that cause the exclusion of black or brown from a part of the hair, the qualitative effect of the agents appears the same, but the quantitative effect varies. To be concrete, all the agouti animals have a factor which restricts black or brown in the subapical band of the ticked hair, but the amount of this restriction differs, particularly when a wild agouti or a hybridbearing wild agouti is contrasted with the tame. The common qualities of all agoutis are as follows:

(1) All restrict black or brown on the individual hairs in the subapical band, giving each dorsal hair a barred appearance.

(2) Any agouti expresses itself more powerfully on the belly than on the back, restricting black more in this region.

(3) Any agouti is epistatic to the non-agouti condition, and allelomorphic to the absence of agouti.

But to class all agoutis together, without a thorough consideration of their differences, would be a superficial method of treatment. From an examination of many tame agoutis the conclusion is reached that these never show the condition which the wild agouti presents in some pure wild animals and in some hybrids. These differences are briefly as follows:

(a) The very weak restricting power, which some wild individuals and some hybrids show, is unknown in tame guinea-pigs. This difference in the restricting power may be readily seen from measurements of the yellow subapical band, for the greater the power to restrict black or brown, the broader the yellow band from which these pigments are excluded. The narrowest yellow band on a mid-dorsal hair of a tame agouti animal measures about $2 \mathrm{~mm}$. The yellow band of a hybrid or wild agouti may measure as small as $1 \mathrm{~mm}$. In a number of cases the wild agouti was so powerless to restrict black in young hybrids that yellow was not visible at all in the dorsal hairs, and only very slightly 
visible on the belly. Such animals show an extremely slight sprinkling of agouti hairs when they become adult (figs. 6 to 9).

(b) No tame agouti guinea-pig, to my knowledge, has ever shown a ticked belly, by which term I understand a condition in which the individual hairs are barred with yellow and have black tips and bases (figs. 4 and 5). I do not mean that all wild C. rufescens individuals and all wild hybrids are a very black agouti with ticked bellies. Such is not the case. The agouti pattern in the wild, and in hybrids receiving agouti from the wild, varies from a form very closely comparable to the tame to forms almost indistinguishable from black, the latter occurring only in the hybrids.

\section{Modification of the Wild Agoutr.}

To leave comparisons and return to the wild agouti pattern, it may be said at the outset that we do not know how the different shades of wild agouti are inherited when the wild $C$. rufescens individuals are mated inter se. The wild were animals that would not bear much handling, and so our records simply state that they were of the agouti pattern, with some additional data such as "dark" or "light." They could not be classified as so many distinct forms, for their range was great. However, it would have been desirable to know if the darker forms were hypostatic and whether any forms could have been gotten which breed true to one shade as far as could be detected by our crude methods of classifying by visual inspection.

The apparent confusion and contradictions were only increased when the wild were mated to non-agoutis to produce $\frac{1}{2}$ wild hybrids, heterozygous in agouti. Although these animals were heterozygous in the agouti factor (each one having received its share of agouti from one gamete, coming from the wild sire), they produced both dark and light agoutis of various shades in addition to recessive non-agouti offspring. All of the female wild hybrids were mated to non-agouti males up through the matings of the $\frac{1}{4}$ wild; hence we are sure of the source of the agouti in every case, and no admixture of tame agouti could have occurred. The $\frac{1}{4}$ wild females also produced both dark and light agoutis, irrespective of whether the mother was dark or light. As the wild agouti was being passed from one generation of hybrids to the next more dilute generation of hybrids, one fact stood out very clearly. Weaker agoutis gradually made their appearance; in fact, so weak was the agouti becoming that it failed to restrict black altogether dorsally and only very slightly on the belly in some cases (see figs. 6 to 9 ). This weakening of the power of the agouti factor can not be attributed to the fact that the wild agouti is always less potent to restrict black, which comes wholly or partly from the guinea-pig source; for, as has been stated, some hybrid females with strong agouti produced young with weak agouti, and vice versa. 
To put this matter in concrete form, table 13 has been drawn up. In this table all mothers and young are classified as ticked-bellied, dark-bellied, or light-bellied animals. The correlation existing between the ventral and the dorsal sides allows the inference that the shade of agouti on the back of animals classified as ticked is the darkest, whereas the back of animals marked light is the lightest, and an intermediate category, dark, falls in between these two. The animals which had the hair on the belly barred with yellow, but with hair-tip and base dark, were called ticked-bellied, and these animals were the darkest hybrids, both dorsally and ventrally. A few animals were called darkbellied which had hair on the belly that was yellow at the tip but had much black at the base. Those animals in which the hair on the belly was entirely yellow or yellow with little black at the base were classified as light-bellied. These last were the lightest animals dorsally and ventrally and resemble the domestic guinea-pig closely. All the mothers were heterozygous in agouti, having received their agouti factor from one parent, the wild, or the wild hybrid. They were mated to nonagoutis and produced equal numbers of agouti and non-agouti offspring, and have been discussed in this light under tables 6 to 11 . Now, table 13 shows that these same agouti offspring were of variable character. The recessive non-agouti offspring are here disregarded.

The Cavia rufescens had been mated with guinea-pig females, and yielded all agouti offspring. The records show that 11 were very dark with ticked bellies, 1 dark with dark belly, and 2 light with light bellies. Just what the rest were can not be told, for they died young or were aborted. The $\frac{1}{2}$ wild used as mothers of the $\frac{1}{4}$ wild had ticked bellies, and are entered on the first line of table 13 . In spite of their dark color they produced only 18 like themselves ( 43 per cent), 5 intermediates, and 19 light agoutis. The $\frac{1}{4}$ wild with ticked belly transmitted their character to a large proportion (90 per cent) of their offspring, producing 19 ticked bellies and 2 light. The $\frac{1}{8}$ wild with ticked bellies, and all hybrids thereafter, produced only ticked-belly offspring (100 per cent). Since the construction of the table, new experiments with fertile hybrid $\frac{1}{64}$ and $\frac{1}{128}$ wild males show that these also transmit the very dark agouti with ticked belly to their offspring, irrespective of whether they are mated to $\frac{1}{4}$ wild non-agoutis or to guinea-pig non-agouti or whether they are fathers of $\frac{17}{128}$ wild, or $\frac{1}{128}$ wild, or $\frac{1}{256}$ wild.

The dark-bellied females used were only two in number, both $\frac{1}{4}$ wild animals; one produced a dark-bellied young one and the other a lightbellied one. They evidently do not always transmit agouti just like their own, but nothing can be said further than that.

The light-bellied females also fail to transmit in all cases agouti which acts just as their own; for the $\frac{1}{4}$ wild mothers with light bellies gave 7 ticked-bellied young (41 per cent) and 17 light bellied young. The 
$\frac{1}{8}$ wild, a single individual ( $\$ 140$ ), though light-bellied, produced only young with ticked belly.

Thus it is seen that light-bellied may produce the darkest shade and vice versa. It may be objected that the difference between the lightest and darkest is a small one, and renders close analysis and tabulation impossible. Such an objection is hardly valid when one considers that the darkest forms are often almost indistinguishable from black, whereas the lightest form is almost as yellow as an ordinary golden-agouti guinea-pig (figs. 4 and 5). Whether or not light agouti females would gradually or quickly be replaced by dark ones upon continued crossing with the guinea-pig can not be said, for further crossing of the lightbellied females was omitted at the time and no light-bellied females occurred after the $\frac{1}{8}$ wild generation, but a few light-bellied $\frac{1}{4}$ wild are still alive and, with these, it is hoped to investigate the question further.

It is most perplexing to assign reasons for these various expressions of the agouti factor. One can hardly suppose that the very darkest agouti, which is almost black, possesses precisely the same thing which was contributed by the wild. In some cases ( 075 ) the $\frac{1}{2}$ wild was very dark. In others (as through the series, $\frac{1}{4}$ ㅇ $91, \frac{1}{8}$ ㅇ $723, \frac{1}{16} \sigma^{7} 1082$ ) the change was carefully watched and the transition was noted, but it did not take place in one generation. It might be supposed that the C. rufescens agouti factor has inherently less restricting power in the hybrids than in its own species, but this explanation obviously will not apply to those hybrids which are light-bellied, nor to those cases in which a gradual loss of restricting power was observed to occur in a series of generations. Furthermore, it does not explain why light forms gave both light and dark, just as the dark forms gave dark and light progeny. No matings of any description among tame guinea-pigs have yet made it necessary to postulate a number of similar agouti factors which are coupled. If wild agouti is held to be made up of $A_{1}$, $A_{2}, A_{3}$, . . . $A_{n}$, then it could be supposed that one or a number of these factors dropped out and gave a weaker and darker agouti. This would explain how $q 63$, $q 68, \% 69$, and other $\frac{1}{2}$ wild animals happened to be very dark, because of a weak agouti with less genes; but it would never explain how some of the $\mathrm{F}_{2}$ offspring and all of the $\mathrm{F}_{3}$ offspring of particular females could possibly acquire these lost genes again and become light yellow agoutis with an agouti factor that is more powerful to restrict black. No admixture of tame agouti can be considered a causal agency in the change, since tame agouti hybrids were not produced until the $\mathrm{F}_{4}$ generation.

In analyzing the case, it must be remembered that the Cavia rufescens agouti factor has been acting on Cavia rufescens black for centuries. Whatever agouti is, it is something which determines physiologically a rhythmical deposition of pigments in the growing hair. It is not surprising that such an activator, or whatever it is that is contributed 
by the sperm of the wild male, upon entering the egg of a tame female, should show many strange and unaccustomed reactions, disturbances, and possibly modifications. No one was surprised that Hertwig (1910) could cause crippled embryos to appear by treating frogs' eggs with radium rays, and no one need postulate that such treatment eliminated some of the genes necessary to the normal development of certain organs. And so, the series of reactions which take place in a fairly stereotyped manner, when wild agouti develops in the pure wild race, may well be upset when one or several materials, necessary for this series of reactions, are carried by the wild sperm to such an unaccustomed environment as the egg of another species. This modification of agouti does not vitiate the Mendelizing inheritance shown in tables 6 to 11 , for the material body which carries the agouti factor originally contributed by the wild sperm separates from its homologue, contributed by the egg. The material bodies or carriers (possibly chromosomes) separate. The activator of the rhythmic deposition of pigment in the hair, the agouti factor, residing in one of these carriers may have been modified or unmodified; yet, modified or unmodified, it separated from its allelomorph.

Summarizing the facts observed:

(1) Each $\frac{1}{2}$ wild hybrid received a single dose of agouti from a wild male; 11 of the $14 \frac{1}{2}$ wild were dark with ticked bellies, and varied from forms much darker than the wild to forms like the darkest wild.

(2) This modification shown by some $\frac{1}{2}$ wild females was present in their offspring for the next successive six generations. In some cases the agouti gradually became darker, but in others the change took place more quickly.

(3) The modification shown by other $\frac{1}{2}$ wild females did not persist in all cases, for they produced light individuals as well as very dark ones. When light $\frac{1}{4}$ or $\frac{1}{8}$ wild forms were thus produced, these gave rise either to very dark forms again or to light forms. When dark $\frac{1}{4}$ wild were produced they also gave dark and light offspring.

Disturbanees which quite baffle the cut and dried Mendelian interpretation are not unknown in wide crosses. Not only do we find metabolic disturbances, as in the echinoderms and insects, but in cases where adults have been raised there often occur gynandromorphs, hermaphrodites, and the like (Standfuss, 1895). Up to the present time the mitoses of the hybrid germ-cells in these crosses have not been given the study which they deserve, and consequently an intimate acquaintance with internal mitotic phenomena of hybrids has not been formed.

\section{Modified Wild Agouti in Crosses.}

Irrespective of the uncertain manner in which the agouti character expressed itself in the first three hybrid generations, there were some families which consistently gave dark forms for a number of generations, 
and since these were easily distinguishable from the light tame agouti, several crosses were made, into which they both entered, and many more are in progress. Ten different $\frac{1}{8}$ wild females and one $\frac{1}{16}$ wild female were used in the following crosses:

Cross 1: $\subsetneq 247$, $~ 248$, and $\subsetneq 311$ were crossed with guinea-pig males homozygous in agouti.

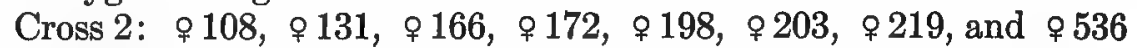
were crossed with male guinea-pigs heterozygous in agouti.

The result of the first cross was a complete dominance of the tame, light, and powerful agouti over the wild, dark, and weak agouti; hence all the young were light yellow agoutis with light bellies. If the wild heterozygous agouti is designated by $A^{\prime} a$, and the tame homozygous agouti by $\mathrm{AA}$, then the gametes formed and zygotes resulting from their union in this cross were:

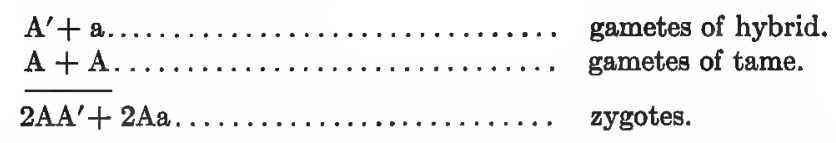

It is evident that in half the zygotes produced are found both kinds of agouti, while in the other half only tame agouti occurs. Since the tame agouti is dominant, all zygotes look alike, but the heterozygous animals should give only tame agoutis and non-agoutis when they are bred to non-agouti animals. Their gametes should be $\mathrm{A}+\mathrm{a}$, and combined with those of a recessive, a $\mathrm{a}$, should give zygotes $2 \mathrm{Aa}+2 \mathrm{aa}$. On the other hand, the animals homozygous in agouti should produce gametes $\mathrm{A}$ and $\mathrm{A}^{\prime}$; and when such animals are mated to recessive nonagoutis, with gametes $a+a$, they can give only young of the two sorts expressed by the formula $A a+A^{\prime} a$; that is, all agouti, but with equal numbers having the dark wild coat and light tame coat. Since only three heterozygous wild agouti females were mated to the homozygous tame agouti males, the number of offspring produced in cross 1 was small. These wild females have already been entered in table 3 . Referring to that table, it will be seen that $q 248$ and $q 311$ produced 5 young, and that $\circ 247$ and $q 248$ appear as possible mothers in cases of doubtful motherhood; in all cases, it may be stated, the offspring were light agoutis with light bellies. When the young produced by cross 1 were tested individually by breeding them to non-agouti mates, they were found to be characterized as follows:

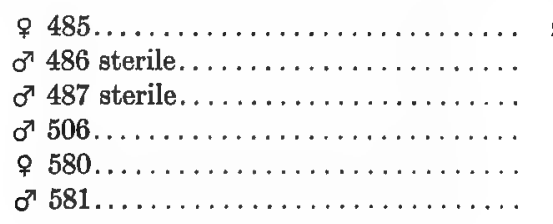

$\begin{array}{ccc}\text { zygotic formula } & \mathrm{AA}^{\prime} \\ " & \text { " } & ? \\ \text { " } & \text { " } & \mathrm{AA}^{\prime} \\ \text { " } & \text { " } & \mathrm{Aa} \\ \text { " } & \text { " } & \mathrm{Aa}\end{array}$


Both the expected zygotic classes are here represented and in the expected equality.

The results of cross 2 are given in detail in table 14. Using the same zygotic formula for the dark, wild, hybrid, ticked-bellied females as was used in cross 1 , their gametes should be $\mathrm{A}^{\prime}$ and a. The males were agouti guinea-pigs with light bellies, but heterozygous. Their zygotic formula would be Aa and they would produce gametes $\mathrm{A}$ and a. ${ }^{1}$

The gametic combinations expected to occur in cross 2 may be expressed as follows:

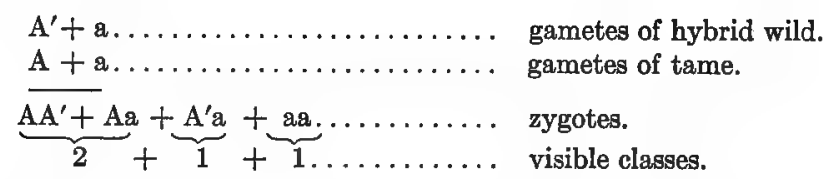

According to this scheme, three visible classes of offspring result. Four real zygotic classes are produced. The first two zygotic classes, $\mathrm{AA}^{\prime}$ and $\mathrm{Aa}$, look alike because the tame agouti is epistatic, as has been shown in table 3 and in the previous discussion. The real difference is shown by breeding these two classes to non-agouti animals. The class $A^{\prime} a$ is composed of animals of the dark wild agouti pattern with ticked belly. The class aa is a non-agouti class. The visible classes should occur in the ratio $2: 1: 1$. The numbers actually recorded are $19: 13: 4$ The most probable expectation would be $18: 9: 9$.

An examination of the breeding records of the visible classes gives the final proof of the actual existence of the expected zygotic classes:

Visible class, $A A^{\prime}+A a$.-These two zygotic classes are alike lightbellied light agoutis, because $A$ is dominant to $A^{\prime}$ and to $a$. The zygotic class, $\mathrm{AA}^{\prime}$, should produce gametes $\mathrm{A}+\mathrm{A}^{\prime}$, and when mated to non-agouti animals should give Aa+A'a; that is, tame and wild agoutis in equal numbers, but no animals without agouti. The zygotic class Aa, when mated to non-agoutis, should give equal numbers of tame light agoutis and non-agoutis. Of course, not all animals could be tested, and the numbers were cut down by the sterility of the males as well as by premature death of some females. The zygotic class, $\mathrm{AA}^{\prime}$, is represented by $q 399$, +448 , and $q 499$, which produced only agouti offspring, but of two sorts, dark wild and light tame, in approximately equal numbers (table 15). The segregation of the two sorts of agouti from each other was complete and definite. No trace of any tainting of one agouti by the other was observable. The dark, tickedbellied young of table 15 were of the darkest shade; the light-bellied young were like a normal agouti guinea-pig. There was no segre-

\footnotetext{
IIt is evident that I am using " $a$ " as the allelomorph of both $A$ " and A. This may need explanation, for it may be urged that the allelomorph of $A^{\prime}$ is $a^{\prime}$; but since $A^{\prime}$ and $A$ are allelomorphic to their absence (tables 6 to 11) and this "absence" is one and the same thing (visibly and in crosses), we may designate this absence by a single symbol.
} 
gation of a separable belly factor, for the usual correlation between the dorsal and ventral sides was observable. The zygotic class, Aa, occurs as expected about as frequently as class $\mathrm{AA}^{\prime}$. It is represented by o 356, $ᄋ 414$, $q 481$ and $\$ 2030$, which were mated to recessive nonagouti males and produced each two sorts of young, light-bellied tame agouti and non-agouti, in equal numbers. The total number of young which they produced was 10 agoutis and 10 non-agoutis, the most probable expectation. These females have already been entered in table 9, and it need only be added that their agouti young were in all cases of the tame agouti type.

Visible class $A^{\prime} a$. - This class is distinguished from the zygotic class, $\mathrm{Aa}$, by its very dark ticked-bellied agouti, which was received from the wild source. It should occur among the offspring of cross 2 as frequently as the class $\mathrm{AA}^{\prime}$ or the class Aa. The actual number produced was 13 , whereas the most probable expectation is 9 . Like the class $\mathrm{Aa}$, it is heterozygous in agouti; and, when mated to non-agoutis, produces equal numbers of agouti and non-agouti offspring; but the agouti offspring are all of the dark, ticked-belly type. Of the 13 animals assigned to this class on the basis of visible characters, it was possible to test 5 females and 1 male. Female 195, $q 421$, ㅇ 565, and ${ }^{7} 412$ were mated to non-agouti guinea-pigs, and produced 8 dark, tickedbellied agoutis and 14 non-agouti young. The most probable expectation is a ratio of $11: 11$. The females have already been entered in table 9 , and it need only be added that the agouti produced were of the modified wild agouti type. Female 357 and $\$ 484$ were tested with non-agouti males, but their young are so few that the test is inconclusive; they produced 3 tick-bellied young and 1 albino, but no non-agoutis, hence their supposed heterozygous character as regards agouti has not been demonstrated.

Visible class aa.-This non-agouti class consisted of 4 individuals, whereas the most probable expectation is 9 . With such relatively small numbers as must content the breeder of mammals, it is sufficient to know that the class occurs. The animals which made up this class were sterile males or died prematurely. There is no reason to believe that, if they had been tested, they would not have acted just as any other non-agouti recessives. Having raised over 400 young from nonagoutis bred to non-agoutis and observing no exception to the rule that these recessives breed true, whether they are guinea-pigs or hybrids, it is safe to assume that class aa is exactly what it appears to be, pure non-agouti.

\footnotetext{
"Presence and Absence" Hypotaesis Applied.
}

In the foregoing discussion, wild agouti and tame agouti are considered allelomorphic to each other. This hypothesis seems unavoidable, for, if the two sorts of agouti behaved as units wholly independent 
in heredity, then the hybrids containing both sorts of agouti, each in a single dose, should produce some non-agouti offspring when mated to non-agoutis, but they do not. These hybrids (whether received from Cross 1 or from Cross 2) would on that assumption have a formula $\mathrm{AA}^{\prime} \mathrm{aa}^{\prime}$ in place of $\mathrm{AA}^{\prime}$ and should produce four kinds of gametes in equal numbers, viz, $\mathrm{AA}^{\prime}, \mathrm{Aa}^{\prime}, \mathrm{A}^{\prime} \mathrm{a}$, and $\mathrm{aa}^{\prime}$. One-fourth of the gametes in that case should fail to transmit agouti; but the experimental evidence given in tables 4 and 15 shows clearly that such is not the case, and that, therefore, the hybrids produce only two kinds of gametes, one of which carries tame agouti, while the other carries wild agouti. Hence, the hypothesis that wild and tame agouti are not allelomorphic is untenable, at least in the simple form stated.

Nevertheless, it may be assumed that the dominant agouti of the tame guinea-pig really contains the same agouti as C. rufescens, but has an additional differential factor, D, firmly coupled with it. The tame agouti in that case might be designated by the inseparable combination $\widehat{A^{\prime} \mathrm{D}}$, which is the equivalent of $\mathrm{A}$ in the foregoing pages. The wild agouti would then be designated by $\widehat{A^{\prime}}$. The two would be allelomorphic to each other and each to its absence, ad. This explanation does no violence to the observed facts, but is untenable without the supplementary hypothesis of coupling. This line of explanation does not simplify the statement that wild agouti and tame agouti behave as allelomorphs to each other, although it allows one to account for the origin of "wild" agouti from "tame" by a break in the supposed coupling. It also has advantages from the standpoint of those who consider unit characters unchangeable, except by the addition or subtraction of distinct factors likewise unchangeable. This method of procedure, however, encounters difficulty in explaining how wild, lightbellied, light agouti of the early hybrids might give dark, ticked-bellied agouti and then these latter give the former again.

Fewer suppositions make the first alternative explanation simpler, for any sort of agouti is allelomorphic to any other sort of agouti in guinea-pigs. The segregation of the modified dark, ticked-bellied agouti from the light agouti is more apparent and striking on account of the differences. That they do segregate may be due to their being carried in homologous chromosomes.

Morgan (1911) and Cuénot (1911) have described light-bellied agouti mice which are dominant to the gray-bellied variety. The two forms segregate. Hurst (1905), on the other hand, mated a very yellow agouti rabbit to an albino, and got the darker wild gray type. $\mathrm{He}$ reports that no segregation occurred.

My experience with the transmission of the wild agouti factor to the hybrids and its inheritance is as follows:

(1) The wild agouti, when transmitted to the hybrids, may give a very dark ticked-bellied coat. This modification may persist, become 
accentuated, or be lost. In the early generations of hybrids it acted in no uniform manner, but seemed to vacillate. The majority of the hybrids tended toward the very dark type. This can not be held to be the ultimate course for all the progeny, because no light-bellied hybrids were bred after the $\frac{1}{3}$ wild. Had such occurred and been bred, it is possible that some progeny might have remained of the practically unchanged wild agouti pattern, to which some $\frac{1}{4}$ wild animals had reverted.

(2) When one crosses the modified, dark, ticked-bellied agouti with tame, light-bellied agouti, the latter is epistatic and both forms segregate from each other in the $F_{2}$ generation. Both sorts of agouti are allelomorphic to their absence, and also to each other. ${ }^{1}$

\section{NON-AGOUTIS MATED INTER SE.}

Extracted non-agouti hybrids appeared in the $\mathrm{F}_{2}$ generation (see table 6). Other similar, extracted recessives appeared in seven subsequent generations. They have all bred true when mated to recessive mates and have given, up to the time of tabulation, about 400 nonagouti offspring. This agrees with the experience of other observers, that extracted non-agoutis breed true to the non-agouti character. This applies to matings of hybrid females and hybrid males, and hybrid males with guinea-pigs, as well as hybrid females to guinea-pigs. The cumbersome tables for this class of matings are not given, inasmuch as the result is fairly obvious and any deviation would mean an unexpected reversal of dominance.

\section{BLACK AND BROWN. HOMOZYGOUS BLACKS IN CROSSES.}

Black, in guinea-pigs and mice, is epistatic to brown. Wild gray mice and ordinary agouti guinea-pigs are homozygous in black. Rabbits and rats are likewise homozygous in this factor, but we know of nobrown in the latter two. The wild Cavia rufescens as bred in the laboratory (table 1) acted just as a wild mouse or pure strain of agouti guinea-pig. All the offspring were black-pigmented and agouti-marked. Among the later generations of hybrids not all black-pigmented young were agouti-marked, but for our present purpose the two are included in a single classification, since both bore black pigmentation.

When a wild male was mated with female guinea-pigs of any color or of no color, the offspring were black pigmented. This result shows that the wild males are homozygous in black. Matings of this sort, summarized in table 2, produced 37 young, all of which were black agouti. These $\frac{1}{2}$ wild individuals produced 83 black-pigmented offspring when mated with guinea-pigs of various colors (see table 6).

\footnotetext{
${ }^{1}$ It should be added that the wild modified agouti could be recombined with brown, giving ticked-bellied cinnamon agoutis. The formula would be presumably $A^{\prime} A^{\prime} b b$ or $A^{\prime} a b b$.
} 
Among the guinea-pig males used in these matings were 3 brownpigmented individuals, viz, ơ $617, \sigma^{7} 9246$, and $\sigma^{\top} \mathrm{NW}$, which sired in all $24 \frac{1}{4}$ wild offspring. These, though black-pigmented like the $\frac{1}{2}$ wild mothers, were heterozygous for that character, and would therefore be expected to transmit black in only half of their gametes, the remainder transmitting, instead the recessive condition, brown. The sequel justified this expectation, as we shall see.

The experiments with homozygous black may be divided into two groups, both of which produce only black young. The groups are:

(1) Matings of hybrid females, homozygous, heterozygous, or lacking black, with guinea-pig males which were homozygous in this factor.

(2) The reciprocal crosses in which the guinea-pig males were heterozygous or lacking black, but the female hybrids were homozygous in this factor.

Tables 16 to 20 give all such matings from the $\frac{1}{4}$ wild up through the $\frac{1}{64}$ wild. The summary of all these matings is given in table 21 , showing conclusively that black is epistatic to brown, irrespective of whether the male guinea-pig produces brown gametes, as in matings recorded in tables 6,17 , and 18 , or whether the female hybrids produce such gametes as in the matings recorded in tables $16,17,18,19$, and 20. The total number of young, from such females of 6 different blooddilutions, is 680 (table 21). In not a single case was there any reversal of dominance, every cross giving black offspring when expected. For instance, in the matings recorded in table 16,5 females (o 90 , 우 91 , o 107, $\% 115$, and $\% 124$ ) gave only black young, but they proved their heterozygous nature in matings recorded in table 22 by producing some brown young when mated to a brown male. All heterozygous females and guinea-pig sires in this experiment were similarly tested.

The result of all these matings is precisely what would be expected of similar matings involving homozygous black in guinea-pigs, as far as the qualitative character of black is concerned; nevertheless, extremely dilute forms of black arose in matings of brindled males to a number of $\frac{1}{16}$ wild hybrid females. These males carried black in a homozygous condition; but, as far as was known, carried no dilution factor. Matings are to be made which will show whether the males really carry such a factor or whether the extreme dilution is but another case of unexpected disturbance or modification in a factor which has been held to be fairly uniform.

\section{HETEROZYGOUS BLACKS MATED WITH BROWN.}

Retrogressing for a moment, it will be recalled that brown males 617,9246 , and NW were mated with some homozygous black $\frac{1}{2}$ wild females, producing 24 black offspring, presumably heterozygous. To establish a race of brown hybrids, 14 of these $\frac{1}{4}$ wild offspring were mated to brown males (table 22). The total number of young pro- 
duced was 102 , of which 57 were black and 45 were brown; the most probable expectation would be $51: 51$, but a deviation of 6 individuals is not significant. The black offspring were heterozygous, as was to be expected. This is shown by matings recorded in table 23 . The brown offspring were recessive and produced only brown offspring when mated with brown individuals (see table 27 ).

Tables 23 and 24 give all the rest of the matings, similar to those of table 22, for the $\frac{1}{8}, \frac{1}{16}$, and $\frac{1}{32}$ wild females. Both classes of young, black and brown, are expected in about equal numbers from these matings. The actual ratios show both classes produced in proportions which do not deviate farther from equality than similar matings among guinea-pigs. The total numbers were 109 black and 85 brown (table 25 ). There was an excess in favor of the dominant factor, but not of significant size, I believe.

\section{HETEROZYGOUS BLACKS MATED INTER SE.}

Two matings of this sort were made, which produced 7 black young and 1 brown, the expected ratio being $3: 1$ (see table 26).

BROWNS MATED INTER SE.

It was stated that brown hybrid $\frac{1}{8}$ wild offspring were obtained by mating heterozygous blacks with brown males (table 22). Thus a brown race of hybrids was obtained in two generations, by the application of Mendelian principles, for first homozygous black $\frac{1}{2}$ wild females were mated to brown males, and then their offspring were again mated back to brown, producing brown as well as black offspring. A number of the brown hybrids were used in experiments already described, to prove the dominance of black over brown in these crosses. The rest of the brown hybrids were used in the experiments tabulated in tables 27 and $28 ; 14$ brown $\frac{1}{8}$ wild females were mated to brown males, producing 78 brown offspring; 13 of the $\frac{1}{16}$ wild, and 1 of the $\frac{1}{82}$ wild were similarly mated. The results are clear; a brown wild hybrid female produces gametes bearing only brown, $b$. We know that the guineapig males do the same. The zygotes, resulting, are bb in formula, i. e., homozygous brown. The summary given in table 29 shows that 111 brown offspring resulted from these matings. There was no reversal of dominance, for a wild hybrid breeds true to brown just the same as a guinea-pig. The interesting speculation immediately suggests itself: can we produce a brown race which shall be in all other respects identical with Cavia rufescens? To do so would undoubtedly require a long series of matings, since many independent character differences exist between $C$. rufescens and $C$. porcellus. If, however, they all conform with Mendel's law in heredity and the principle of gametic purity holds good in this case, the combination suggested should be capable of realization. 


\section{EXTENSION AND RESTRICTION.}

\section{HOMOZYGOUS CONDITION OF EXTENSION IN CROSSES.}

Guinea-pigs of the varieties known as red, yellow, and cream agree in having no black or brown pigment in their hair, but instead a yellow pigment of varying intensity. Such animals I shall for convenience call "red," whatever the intensity of their pigmentation. The eyes of red guinea-pigs are either black or brown pigmented. Black-eyed reds may transmit black coat-color in crosses, but brown-eyed reds can not do so, though they are capable of transmitting brown coat-color in crosses.

Since the black or brown pigment in a red animal is restricted to the eyes and skin and does not occur in the fur, we may speak of such an animal as restricted black or brown, and the gametes which transmit this condition as possessing a restriction factor. Or, looking at the matter from an equally justifiable point of view, a red animal is considered to lack the factor for extension which, in either a single or a double dose, gives rise to black or brown. They may also carry, unseen, that factor which acts only on black or brown, the so-called agouti factor. Indeed, a number of the animals which have been entered in tables dealing with the transmission of agouti were reds. Similarly albino animals may be legitimately classified as regards their power to transmit color characters, even though they themselves do not manifest those color characters.

No red individuals of wild Cavia rufescens are known. Just as in the case of agouti, and black, in which the wild is homozygous, so, in the case of extension, it was surmised the wild would prove to be homozygous. Two guinea-pig females, known to be heterozygous in extension, were mated to one of the wild males $\left(o^{x} 33\right.$ ) and produced 6 young (table 30), all of which had completely extended black pigmentation. None of the wild, mated inter se, ever gave young with the restricted (red) coat color. It is therefore safe to assume that wild individuals transmit the extension factor in all gametes.

None of the animals produced in these matings was used afterward except $\$ 72$. She proved to be homozygous in the extension factor. This is not at all surprising, for there was an even chance that she would be homozygous or heterozygous. All the other $\frac{1}{2}$ wild females were also homozygous for extension. Six of the $\frac{1}{2}$ wild females were mated (table 31) to guinea-pig males, carrying the extension factor in a heterozygous condition ( $\sigma^{\top} 4, \sigma^{\top} 9246$ ) or lacking it entirely ( $\sigma^{\top} 617$ ). These matings produced 29 offspring, all of which were of the extended pigmentation, thus proving that, in the hybrids, extension is epistatic to restriction, just the same as in guinea-pig matings. The $\frac{1}{4}$ wild, thus produced, would be of two classes, homozygous (EE) and heterozygous (Ee). 
Tables 31 to 36 record all the matings of wild hybrid females, from the $\frac{1}{2}$ wild up through the $\frac{1}{64}$ wild, in which at least one member of each cross was homozygous in the extension factor. Combined, all these matings produced 628 offspring with extended pigmentation (table 37). The conclusion is obvious: extension is epistatic to restriction in hybrids of various blood-dilutions, precisely as among guinea-pigs.

\section{HETEROZYGOUS CONDITION OF EXTENSION CROSSED WITH RESTRICTION.}

Regressing, it will be recalled that 3 guinea-pig males carrying restriction were mated to some $\frac{1}{2}$ wild females. The matings produced 29 offspring (table 31), of which some should be heterozygous. These $\frac{1}{4}$ wild offspring were the first that could be used to establish a red race of hybrids. When two of these were mated with recessive, red guineapig males, they produced red-coated as well as black-coated young, in the ratio $9: 10$ (table 38). This result fulfills the conditions of most probable expectation. It shows clearly that the $\frac{1}{4}$ wild individuals can form gametes of two kinds, one of which bears the maternal character extension, and the other the paternal character, restriction, received from the tame stock.

Two classes of matings (table 39) were made among the $\frac{1}{8}$ wild females, which should yield animals of extended pigmentation and restricted pigmentation in approximately equal numbers:

(1) Female hybrids, heterozygous in extension (produced in matings recorded in tables 32 and 38), were mated to red guinea-pig males.

(2) Red female hybrids, lacking entirely the extended coat (produced in matings recorded in table 38), were mated to guinea-pig males, heterozygous in extension. Similar matings were made among $\frac{1}{16}$ wild hybrids (table 40 ), $\frac{1}{3: 2}$ wild hybrids (table 41 ), and $\frac{1}{64}$ wild hybrids (table 41). The summary of all these matings is given in table 42 . The offspring fall into the two expected classes: (1) animals with an extended coat pigmentation, Ee, yet heterozygous in extension; (2) animals of red or restricted coat pigmentation, ee. The classes should occur in approximate equality. The ratio $47: 55$ is so close to the most probable expectation, $51: 51$, as scarcely to require comment. Segregation as regards the extension factor evidently occurs among the hybrids just as among guinea-pigs. The brown-eyed red guinea-pig represents a combination of three recessive color characters which segregate independently in crosses of one variety of guinea-pig with another. They behaved in the same way in crosses between C. rufescens (or its guinea-pig hybrids) and the guinea-pig. Yet segregation and recombination of these several color characters is without apparent influence on the fertility of the hybrids. One color variety of hybrid is no more fertile than another. 
HETEROZYGOTES FOR EXTENSION MATED INTER SE.

Only 14 matings were made in which both parents were known to be heterozygous in extension (table 43). The wild hybrid females used ranged from $\frac{1}{4}$ wild to $\frac{1}{32}$ wild. The male parent was in every case a guinea-pig. In these matings each parent is expected, on Mendelian principles, to produce, in equal numbers, gametes carrying extension and gametes without that factor. The chance combinations of such gametes should give two visible classes, in the ratio of $3: 1$. The actual results were 45 of extended pigmentation and 13 of restricted pigmentation, which is very close to the most probable expectation. The hybrid females therefore form two kinds of gametes, just as guinea-pigs do; and the usual $3: 1$ ratio results from mating a heterozygous hybrid with a heterozygous guinea-pig.

REDS MATED INTER SE.

The fact having been established that red is a recessive character among the hybrids as among guinea-pigs, it would seem to be scarcely necessary to show by breeding test that reds produce only red-colored offspring. Nevertheless, three matings have been made between red hybrid females (290, 291, and 292) and a red guinea-pig male (67). These matings produced 4 offspring, all red. ${ }^{1}$

\section{COLOR AND ALBINISM.}

HOMOZYGOUS CONDITION OF THE COLOR FACTOR IN CROSSES.

Albinism is common among domesticated rodents. It has been shown to be allelomorphic to color in mice, rabbits, rats, and guineapigs. Recently, Castle (1912) reported a case in which a wild albinic sport of Peromyscus was mated to normals, and by mating a normal $F_{1}$ female back to the albino father, normal and albino $F_{2}$ offspring were obtained. Albinos are not known among any wild cavies. The explanation for the albinic condition on a factorial basis suggested by Cuenot (1903) is now generally accepted. This explanation postulates a color factor, C, which is necessary for the development of color in the eye, hair, and skin; and the entire absence of this factor (designated by c) results in albinism. Among rabbits, two sorts of albinos are recognized, the ordinary and the Himalayan albino. The latter condition is distinct, for a small amount of pigment is present in the hair of the nose, ears, and other extremities; and this condition is epistatic to ordinary albinism. It may be necessary to assume a different factor, such as $\mathrm{C}^{\prime}$, for the Himalayan condition, in place of c, which is used for the ordinary albino. In this case, $\mathrm{C}^{\prime}$ would be allelomorphic to $\mathrm{C}$ or $\mathrm{c}$, just as $\mathrm{A}^{\prime}$ has been shown to be allelomorphic

'Since the foregoing was written a similar result has been obtained from additional matinga, in some of which the male parent was indeed a fertile hybrid. 
to $\mathrm{A}$ or a in the modified wild-agouti crosses. Albino individuals occur in nature from time to time in many species, but it is supposed that their conspicuousness in most cases renders them an easy prey to their enemies. Albino guinea-pigs are always Himalayan.

The old original wild male ( $\left.\sigma^{7} 1\right)$ was bred to three albino guinea-pig females; his son ( $\sigma^{7} 33$ ) was also bred to an albino. Altogether such matings produced 18 young (table 44 ), all of which were normally colored. It is probable from this, and from the records of the wild bred inter se, that all of the C. rufescens used in this experiment were homozygous in the color factor. Early deaths and sterility prevented the use of the offspring recorded in table 44, in further experiments; but there is little doubt that the animals thus produced were heterozygous in the color factor, with formula $\mathrm{Cc}$; for in mating other $\frac{1}{2} \mathrm{wi}^{\prime} \mathrm{d}$ semales to guinea-pig ma'es which lacked the color factor, young with a formula $\mathrm{Cc}$, were produced.

A number of $\frac{1}{2}$ wild females which were homozygous in color were mated to guinea-pig males which lacked color entirely or were heterozygous in it (table 45 ), producing 27 colored young. Just as the pure wild C. rufescens color factor is epistatic to its absence in the guineapig, so the $\frac{1}{2}$ wild which had received one dose of the color factor from C. rufescens and one dose from C. porcellus were dominant in crosses.

Table 46 shows the complete dominance of color over the albinic condition in all the remaining blood-dilutions. In these matings, one parent was homozygous in the color factor and the other was an albino or carried albinism. The matings produced 252 colored young; and if these are added to tables 44 and 45 , the grand total of 297 colored young shows quite conclusively that the color factor of the wild $C$. rufescens, the hybrids, and the tame guinea-pig is epistatic to its absence, irrespectively of the sort of animal which presents the "absence." It is also obvious that some hybrids, in addition to the $\frac{1}{2}$ wild, must carry the color factors of the wild and of the tame together, but no distinction is visible. Heterozygotes must also occur which received their single dose of the color factor in some cases from the wild, in others from the tame, if we are to believe that the two segregate and keep their identity in the same way that the dark modified agouti factor does. The same reasoning should hold true for black, brown, and extension, but no visible difference in the case of these factors can be detected any more than in the case of the color factor itself.

\section{HETEROZYGOUS COLORED ANIMALS IN CROSSES WITH ALBINOS.}

A number of matings were made in which female hybrids of various blood-dilutions, from the $\frac{1}{4}$ wild up through the $\frac{1}{3 *}$ wild, but heterozygous in the color factor, were mated with male albino guinea-pigs. Reciprocal crosses were also made, in which the female hybrids were albinos and the male guinea-pigs were heterozygotes. Matings of this 
description should produce about equal numbers of colored and albino young.

Since all the young in the matings of table 44 died prematurely no $\frac{1}{2}$ wild which were heterozygous in color could be used for experimentation; therefore albino guinea-pigs or guinea-pigs which were heterozygous in color were mated to a number of the available $\frac{1}{2}$ wild in order to eventually produce a race of albino hybrids. Such matings have been described in table 45 , and the heterozygous colored young from these matings enter as parents into tables 47 and 51 . Table 47 records the matings of two females, heterozygous in color, with albino guineapig males. Each female proved her zygotic formula to be Cc, because she produced both sorts of young. In all, 16 colored and 8 albino young were born, the most probable expectation being 12 of each kind.

Tables 48 and 49 record the remaining matings of the wild hybrid females, from the $\frac{1}{8}$ wild through the $\frac{1}{82}$ wild, in which one parent was heterozygous in the color factor and the other an albino. This class of matings should produce approximately equal numbers of colored and albino young. The summary of tables 47 to 49 is given in table 50 and shows that the total number of colored young (51) is only slightly greater than the number of albino young (43). Segregation and recombination of gametes evidently occur in accordance with the laws of chance as in matings among ordinary guinea-pigs.

\section{HETEROZYGOUS COLORED ANIMALS MATED INTER SE.}

We have already alluded to the fact that the heterozygous colored young born from $\frac{1}{2}$ wild females (table 45) enter into tables 47 and 51 . The former table has been discussed. The rest of the $\frac{1}{4}$ wild, which we know to have been heterozygous, were mated to guinea-pig males likewise heterozygous in the color factor (table 51). Both hybrids and guinea-pigs should produce in equal numbers gametes with and gametes without the color factor. The union of such gametes in these matings should give an average of 3 colored to 1 albino young. The actual results agree closely with theoretical expectation, for 10 colored animals and 3 albinos were produced, whereas the most probable expectation is a ratio of $9: 4$ or $10: 3$.

Since the more intense wild-blooded hybrids agree with the guineapig in this class as well as in most other classes of matings, the remaining more dilute-blooded hybrids may be considered in one group. The $\frac{1}{8}$ wild, $\frac{1}{16}$ wild, and $\frac{1}{32}$ wild females which were heterozygous in the color factor and which were mated to guinea-pigs of similar zygotic formula are recorded in tables 52 to 54 . The summary of tables 51 to 54 is given in table 55. The total number of young from the matings of hybrids, heterozygous in color, with guinea-pigs of the same character, was 119 , of which 80 were colored and 39 were albinos. There is here an excess of the recessive class, for the most probable expectation 
is a ratio of $89: 30$ or $90: 29$. The excess is between 9 and 10 individuals. Nevertheless, it is interesting to note that there was an excess of dominants in the ratios obtained by mating heterozygotes to albinos (table 50); hence the excess of recessives in one case offsets the excess of dominants observed in the other.

\section{ALBINOS MATED INTER SE.}

No matings were made of albinos with albinos. It is safe to assume that the albino hybrids would breed true and agree with the guineapigs in this class of matings, as they do in all other classes of matings. The very fact that a hybrid which is heterozygous in the color factor can form pure gametes of two kinds would be strong argument that albinos breed true. Extracted recessive albino hybrids in previous tables have given no evidence of producing gametes with the color factor when they were mated to guinea-pig males heterozygous in color. ${ }^{1}$

\section{ROUGHNESS AND SMOOTHNESS.}

\section{HOMOZYCOUS ROUGH ANIMALS IN CROSSES.}

It has been often stated that domestic varieties are commonly derived from the wild by the loss of one or more factors; hence the wild is the dominant form, since the presence of a factor is epistatic to its absence. The rough coat of the domestic guinea-pig seems to be an exception to this apparently rather general rule, for the rough character is not found in any wild cavies, yet it is a progressive dominant variation. The rough or rosetted condition of the coat in guineapigs is subject to much variation, but whenever a homozygous rough animal is mated to a smooth one all the offspring show the rough character, and by mating the $\mathrm{F}_{1}$ generation inter se the smooth form can be extracted in the $\mathrm{F}_{2}$ generation. The number of experiments on the wild hybrids which involve the rough coat character are few; nevertheless the numbers are large enough to be significant, particularly since the inheritance of this character in guinea-pigs has been shown to be Mendelian.

Two homozygous rough male guinea-pigs were mated to three female hybrids (table 56) and yielded 10 rough offspring. Two of the females used as dams were smooth, $\frac{1}{8}$ wild hybrids, and the other was a heterozygous, rough, $\frac{1}{82}$ wild hybrid. The total number of 10 rough young would be far too small to serve as a basis for any generalizations, if we did not have reason to suspect that the hybrid and guinea-pig transmit the same characters in a similar manner. Since we know this to be a fact for the other characters which we have already considered, the

\footnotetext{
${ }^{1}$ Since this statement was written fertile male albino hybrids have been mated to fermale albino hybrids and have produced only albino young.
} 
preponderance of probability would allow the same conclusion in this case; hence it is not an unreasonable assumption to conclude that the total of 10 rough young from these matings corroborates a fact which has been firmly established by 249 rough offspring in experiments on the guinea-pig (Castle, 1905). In this light it would have been surprising if the rough guinea-pig males had not shown themselves dominant.

\section{HETEROZYGOUS ROUGH ANIMALS CROSSED WITH SMOOTH ANIMALS.}

The method of procedure in the discussion of color characters has been to consider first the homozygous form of a character in crosses, and since the wild is homozygous in all characters except roughness, the chronological sequence of crosses has heretofore been fairly parallel with the order of discussion. In the case of roughness this is not so, for the wild form was not mated to any homozygous rough animals; hence the discussion began with dilute-blooded hybrids in table 56. Nevertheless the experiments with the rough character were the very first in order of time, for the two female guinea-pigs which were first mated to a wild male $\left(\sigma^{\top} 1\right)$ were heterozygous rough animals. If these two females, $ᄋ 1125$ and o 1625 , had been mated to a smooth guineapig male they would have produced about equal numbers of rough and smooth animals. When mated to the wild male they did precisely the same, for half their gametes carried the rough and half carried the smooth character, whereas all of the wild gametes produced by $\sigma^{7} 1$ carried only the smooth character, and the union of such gametes resulted in 4 rough and 7 smooth offspring (table 57). The departure from the most probable expectation is 1 or 2 individuals.

This result would indicate that mating a smooth wild $C$. rufescens with a rough tame guinea-pig gives the same result as similar matings among guinea-pigs. In a measure it is true. The wild do not carry roughness, and the tame guinea-pig has acquired a progressive dominant variation, but the dominance of this rough character is very incomplete. The $\frac{1}{2}$ wild offspring from the two matings showed a degree of roughness which would almost escape attention. Just a very slight ridge of reversed hair on the back or even only a few reversed hairs on the toes was all that would indicate the maternal contribution. Just exactly why the smooth wild thus inhibits the full expression of a dominant tame rough character must be a matter of conjecture. A similar behavior of the rough character in crosses with certain smooth guinea-pig individuals was noted by Castle (1905).

With the gradual reduction of wild blood in later hybrid generations, the rough character of the hybrids reached the full number of rosettes which is seen in the tame. It may be misleading to state it in that 
way, for the reduction of wild blood and the increase of tame blood may not have any causal relation to the subsequent change in the expression of the rough character. If we represent the factor for roughness by $\mathrm{Rf}$, and the factor for a tame smooth coat by rf, but the factor for a wild smooth coat by $\mathrm{rf}^{\prime}$, then the gametes and zygotes of the animals in table 57 are as follows:

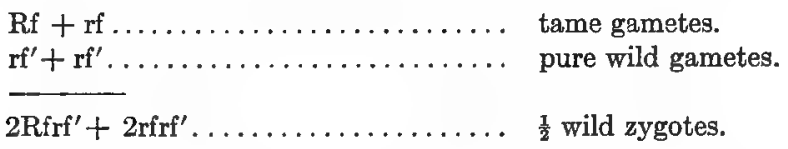

The smooth character of the wild may be due to something slightly different from that of the tame, hence the combination $\mathrm{Rfrf}^{\prime}$ is different from the tame heterozygous rough coat, Rfrf. Now, since the hybrids in these experiments are constantly mated back to smooth guinea-pigs, the great majority of hybrids must eventually carry the guinea-pig's peculiar factor or factors for smooth coat (rf); hence, when the few later dilute hybrids are used, the zygotic formula is probably Rfrf. This means that these later hybrids would be a combination of the rough character and smooth character, both derived from the tame source; and since both are derived from the tame source, the rough hybrids are just like the rough guinea-pigs. In other words, the almost complete inhibition of the rough coat, which the $\frac{1}{2}$ wild hybrids show, is due to the smooth wild parent; but in later generations the smooth character of the wild race is not likely to be present, and the hybrids have the smooth character of the tame. Nehring (1894) must have had a somewhat similar experience with the rough character when he mated a rough guinea-pig to $C$. aperea. His records would indicate a failure of complete dominance; but just what the degree of roughness was can not be stated, for he makes no detailed description of the hybrids as regards roughness.

Table 58 records the rest of the matings of hybrid females with guinea-pig males, in which one parent is heterozygous in roughness and the other parent is smooth. In either case an approximate equality of rough and smooth young is expected. In the first case, in which the guinea-pig male is heterozygous in roughness, 8 rough and 6 smooth were born. In the second case, in which the female wild hybrid was heterozygous in roughness, 19 rough and 20 smooth were born. The total, $27: 26$, is as close an approximation to equality as is possible in an odd number of offspring. If the results of table 57 are added to these, the grand total is 31 rough and 33 smooth wild hybrids. The most probable expectancy is $32: 32$. The hybrids therefore produce equal numbers of gametes which carry the rough factor and which 
lack it, just as a heterozygous rough guinea-pig has been demonstrated to do.

No further matings of rough animals were made. It maybe expected that heterozygotes mated inter se would produce 3 rough : 1 smooth.

\section{SMOOTH ANIMALS MATED INTER SE.}

Without giving tedious tables, it may be stated that at least 1,500 smooth-coated hybrids have been born from smooth animals mated inter se. These range from the $\frac{1}{2}$ wild through eight subsequent generations. All smooth recessives extracted from rough crosses have also bred true; there is no reversal of dominance, even though the rough guinea-pig is very incompletely dominant over the smooth wild C. rufescens.

\section{OTHER COLOR AND COAT CHARACTERS.}

\section{UNIFORMITY AND SPOTTING.}

In guinea-pigs, the dominance of the uniform or self-colored coat over a spotted coat is not so clear and well marked as the dominance of other epistatic characters over their allelomorphs, nor is the segregation of self-colored and spotted coats in the $\mathrm{F}_{2}$ generation perfectly evident.

Rabbits likewise do not show a complete dominance of self-color over Dutch markings; but Hurst (1905) reports that segregation takes place, giving a ratio of 1 self : 2 imperfect dominants : 1 Dutch marked. If, in rats, we consider the hooded pattern as a sort of spotting, then its allelomorph is dominant and segregation is clear, though not complete. In mice, the self-colored varieties are held to be dominant to spotted varieties and segregation takes place, but Miss Durham $(1908,1911)$ has recently reported a "piebald" type which is dominant over selfcolor. The whole question of spotting and its inheritance in guinea-pigs is more unsettled than in any of the other rodents.

Among guinea-pigs, two kinds of spotting are known. They are, (1) the brindled type, in which black, red, and sometimes white hairs are scattered over the body in a sprinkled fashion; (2) the ordinary spotted varieties, in which uniformly colored spots of considerable size occur on the head, shoulder, side, and rump. The spots in this latter type may occur on one or a number of these regions. Since the purpose of this paper is to compare the hybrids with tame guinea-pigs, I shall only attempt to show that similar varieties of spotted hybrids can be produced in both cases.

The wild C. rufescens were all self-colored. In mating the wild males to the tame female guinea-pigs three spotted dams were used. The matings resulted in 5 self-colored $\frac{1}{2}$ wild hybrids. Of these $\frac{1}{2}$ wild hybrids, only 2 were bred ( $\$ 75$ and $q 118$ ). One, $q 118$, was bred to 
an albino male guinea-pig which has spotted ancestry, and she gave 2 spotted and 2 self-colored young, and possibly a third spotted young in a case of doubtful motherhood. The other, $\$ 75$, bred to selfcolored males gave 4 self-colored young. Four other $\frac{1}{2}$ wild females ( +63 , $\circ 68$, $\circ 69$, $\subsetneq 253$ ) were bred to brindled or spotted male guineapigs, but their 40 offspring were self-colored. It would, therefore, appear that the self-pattern of the wild and the $\frac{1}{2}$ wild was dominant to spotting.

When the $\frac{1}{4}$ wild females, which we know had a spotted father, were mated to a pure race of spotted guinea-pigs, they produced 28 selfcolored and 18 spotted young. If dominant self-color and recessive spotting were clearly allelomorphic, then we should expect an approximate equality. There is an excess of self-colored young, nevertheless the spotted variety of hybrids was produced by the admixture of spotting from the guinea-pig source. The clear dominance of the wild self-pattern and that of the $\frac{1}{2}$ wild was lost in the later generations when the hybrids were continually mated back to the guinea-pig. In this and other respects these later hybrids resemble the guinea-pig itself, for dominance of self over spotting is incomplete in pure guineapig races. Both brindled and spotted varieties of hybrids were produced as early as in the $\frac{1}{4}$ wild, the $\mathrm{F}_{2}$ generation.

\section{INTENSITY AND DILUTION.}

In rabbits and mice, a dilute condition of pigmentation is known. This condition is hypostatic to the ordinary intense pigmentation. Black, brown, and red become "blue," light brown, and cream, respectively, when the dilute condition is present. This condition in guineapigs is a distinct recessive factor, for if a cream and a blue are mated, the offspring are blue; but if a red and a blue or a cream and black are mated, only black offspring result. Dilute-pigmented guinea-pigs, mated inter se, breed very true. Whether or not the intense and dilute conditions in guinea-pigs are allelomorphic to each other is a difficult question, but apparently they are.

In the different races of hybrids, dilute animals have appeared. No complete study of such hybrids has been made, for the number of reliable cases is small, yet the fact that such dilute hybrids can occur, just as in the guinea-pig, is certain. An apparent complication has arisen in the case of the hybrids. As has been stated in the discussion of the inheritance of black, there have occurred extremely dilute forms which were not expected. The same is true of brown and cream. No reason for the appearance of these very dilute hybrids can be assigned. They are as light as any which have been obtained in guinea-pigs by continued selection. Curiously enough, most dilute hybrids have appeared when a particular strain of guinea-pig sires was used. These 
sires belong to a brindled race, but are not known to carry dilution. This brindled race of guinea-pigs produced reds, but no creams, when mated inter se. It is not a matter of certainty that the brindled males are entirely or even partly responsible for this extreme dilution. No solution has yet been possible. It is possibly another of the unexpected disturbances which hybrids are prone to show, but for which we know no cause. Cavia rufescens itself was of intense pigmentation.

\section{LONG HAIR AND SHORT HAIR.}

The pure wild stock was short-haired. No experiments have been made to test whether this short-haired condition of the wild is dominant to the long-haired condition of the tame, just as is the case in guineapig matings.

One peculiar character may be recorded here. The wild Cavia rufescens has very straight, coarse, bristly hair, which tends to stand erect, particularly on the head and neck (fig. 1). The $\frac{1}{2}$ wild hybrids had hair intermediate in texture between that of the respective parents, but approaching the guinea-pig more nearly than the wild parent. The approximation to the guinea-pig increased in later generations, so that no clear distinction could be made between the hybrids and guinea-pigs in this particular.

\section{THE FERTILE HYBRID MALES IN COLOR CROSSES.}

All the data which have formed the basis for the study of color inheritance were accumulated from the matings of the wild males with guinea-pigs, or from the matings of hybrid females with guinea-pigs. The result has been to establish sets of allelomorphic pairs and dominance and segregation, comparable to that which occurs in ordinary guinea-pig matings. The conclusions are subject to one limitation, for the hybrid females were continually mated to guinea-pigs and no data were presented on hybrids mated inter se. This restriction is not a serious one, for it has been proven that the female hybrids are similar to the guinea-pig in color transmission. Now, it is well known that sex does not affect the gametic or zygotic color formulæ in guineapigs; hence we have assumed that the sex of the hybrids makes no difference, and that the results obtained from female hybrids would be duplicated by those from fertile male hybrids.

Recently, fertile males have been obtained by reduction of wild blood, $i$. e., by continually mating the female hybrids back to the guinea-pig. A fuller discussion of these results will follow; but at this point it is appropriate to discuss briefly the relation of fertile hybrid males to color inheritance. 
By mating fertile males to guinea-pigs and to hybrids of various blood dilutions, progeny of the following classes have been obtained:

\begin{tabular}{|c|c|c|c|c|}
\hline & Males. & Females. & Abortions. & Total. \\
\hline 174 wild... & 1 & 1 & 0 & 2 \\
\hline$\frac{17}{12 \frac{7}{6}}$ wild... & 2 & 1 & 0 & 3 \\
\hline$\frac{1}{8}$ wild... & 15 & 7 & 1 & 23 \\
\hline $\mathbf{3}^{3}$ wild... & 1 & 1 & 0 & 2 \\
\hline 18 wild... & 31 & 43 & 6 & 80 \\
\hline$\frac{3}{84}$ wild... & 7 & 9 & 0 & 16 \\
\hline$\frac{1}{32}$ wild... & 46 & 39 & 0 & 85 \\
\hline i $\frac{3}{2}$. wild... & 1 & 1 & 0 & 2 \\
\hline$\frac{1}{64}$ wild... & 10 & 6 & 6 & 22 \\
\hline 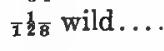 & 3 & 3 & 0 & 6 \\
\hline Totals. . & 117 & 111 & 13 & 241 \\
\hline
\end{tabular}

The number of young, 241, is large, but when these are divided into groups according to the color matings of their parents, there are but few in each class of mating. As far as they go they corroborate entirely the results procured with the female hybrids, previously given. The peculiar, dark agouti (with ticked belly) of the fertile male hybrids is similar also to that of the females in appearance and transmission.

The appearance of these fertile hybrid males is important because of the purely scientific interest in the study of fertility. A number of recent workers have tried to establish fertile male cattaloes. Boyd (1908) has reported on the dominance of the white face of the Hereford, and the polled or hornless condition of the Angus, when crossed with the American bison. The cattaloes lose the valuable coat when they are continually mated to the domestic bull; and the cross with the buffalo bull is frequently fatal to the female cattalo. The success in getting the best quality of bison coats would lie in breeding them together, according to Boyd. However, no half-blood bison are known to be fertile. Iwanoff (1911) and Boyd have reason to believe that the $\frac{1}{4}$ bloods and $\frac{3}{4}$ bloods are fertile. Boyd has successfully bred a $\frac{1}{4}$ blood hybrid bull. Iwanoff has examined the spermatozoa of a $\frac{3}{4}$ blood bison and reports that they are normally developed. He also alludes to a successful mating between such a male and a $\frac{1}{4}$ blood bison female, and supposes that the offspring from this mating should prove to be fertile. This may be the case in these hybrids, but if they are analogous to the wild guinea-pig hybrids, as they seem to be, it would not necessarily follow; for fertile hybrid males and females do not always produce fertile young when mated inter se. Nevertheless, if the cross between the wild and tame guinea-pig is at all comparable to the ungulate species crosses, it is important to know that the same laws of color inheritance obtain in crosses between the hybrids as in the crosses of hybrid females to the parent stock. This would apply to such 
hybrids as the mule, zebroids, zebrules, and the like, if fertile males of these classes of hybrids can be established. That fertile males can be produced among these hybrids, also, is not a matter of certainty, but since the female mule is reported to be occasionally fertile (Waldow von Wahl 1907, Przibram 1910), it may be possible to obtain fertile male mules (Detlefsen 1912).

The detailed discussion of fertility will be given later. The color inheritance of the fertile hybrid males in crosses is the same as that of the females.

\section{GENERAL CONCLUSIONS AS TO COLOR AND COAT CHARACTER.}

The ancestry of the tame guinea-pig (Cavia porcellus) is a matter of considerable doubt, but the prevalent opinion, based on historical and morphological studies, considers the Peruvian cavy (Cavia cutleri) as the immediate ancestor. The relationship between the wild Brazilian cavy (Cavia rufescens) used in the foregoing experiments and the tame guinea-pig is a matter of conjecture; but we may rest assured that these two parent species have had no common ancestry for many, many centuries at least. The relationship is distant, as shown by the many differentiating characters and by the sterility involved in the cross.

In the case of the tame-parent species, a number of unit characters are well known, but in the case of the wild-parent species nothing has previously been known with regard to unit characters or allelomorphic pairs, for it was simply recozgnized as a wild, agouti, cavy species. The tame species has many varieties, and in crossing these varieties inter se we see orderly mechanical separations and recombinations of allelomorphic pairs manifested in Mendelian ratios. We know of no varieties in the wild species, and, since it breeds true, the natural inference is that it is homozygous in most of its characters, if not all. Now, in spite of the fact that these two species have been separated by many centuries and thousands of miles, and by certain peculiar mental and physical structures, and in spite of the many difficulties of even obtaining a successful cross, finally two gametes join to form the hybrid zygote. One of these gametes bears, among other things, a certain number of known factors. The other gamete, coming from the wild, was an unknown quantity and one could only theorize from analogy as to its constitution. To be concrete, the ova, coming from the tame, carried in certain matings no agouti factor, but all the sperm from the wild carried it. The hybrid zygote, therefore, carried it in single dose. These contributions of the diverse parent species separated in the next gametogenesis as nicely as in the case of smooth and wrinkled peas, even though one sex in the first two hybrid generations was completely sterile. From time to time doubts are expressed as to whether Mendelian laws hold in the cases of wide crosses. One purpose of these experiments has been to study a wide mammalian 
species cross in the light of this criticism. It is hoped that the foregoing discussion will show that the law of alternative inheritance has obtained consistently through eight generations of hybrids ranging from the intensely wild to the dilute-blooded generations, and in many different kinds of matings.

The real significance of this alternative inheritance is that a character such as wild agouti, the allelomorph of which has been wild agouti for centuries undoubtedly, can without apparent disturbance take up the non-agouti character as its allelomorph, or the tame-agouti character. The same is true of the other coat characters. Whether this is due to the innate nature of the allelomorphic pairs or due to the material bodies which carry the factors can for the present only be a matter of speculation.

The general conclusions are:

1. Cavia rufescens is homozygous in agouti, black, brown, the extension factor, smooth coat, uniformity, intensity, and short hair.

2. Hybrids of any color variety can be produced by mating it to the guinea-pig. The color and coat characters of C. rufescens are dominant in every case, except as regards roughness and texture of coat and possibly the agouti factor.

3. The hybrids have the zygotic color formula which one would expect to obtain by mating a pure agouti strain of guinea-pigs to some other color variety of guinea-pigs.

4. The agouti of hybrids, though always epistatic to the nonagouti condition of the same, is subject to modification as a result of the cross.

5. This modified wild agouti is very distinct from the tame agouti, and is recessive to it. The two segregate clearly in the $F_{2}$ generation. Both are allelomorphic to each other and to their absence. Hybrids were produced homozygous in agouti, yet bearing the wild and the tame agouti.

6. Roughness derived from the tame guinea-pig is very imperfectly dominant over the smooth wild coat. This incomplete dominance is lost in later, more dilute, wild-blooded generations, and the rough coat becomes normally dominant.

7. The uniform coat of the wild is dominant to the spotted coat of the tame. In later generations the hybrids show the incomplete dominance of uniformity over spotting, which is characteristic of the guinea-pig.

8. Any color variety known in guinea-pigs can be produced in the hybrids. Combinations of tame and wild characters can be made, even bringing in such a morphological character as polydactylism from a tame race, together with the peculiar agouti of the wild race.

9. The inheritance of coat and color characters throughout this species cross is in accordance with Mendel's law. It is equally true of matings of hybrids inter se, and of matings of hybrids of either sex with guinea-pigs. 


\section{PART II. GROWTH AND MORPHOLOGICAL CHARACTERS.}

\section{INTRODUCTORY DISCUSSION.}

The success of Mendel's experiments, which led to the discovery of his "law of dominance and segregation," was due in a great measure to the fact that his materials and methods were well chosen. The characters dealt with were simple and well defined. Previous workers had tried to follow too many characters at one time, or characters with much fluctuation. The early work of those who first sought to corroborate Mendel's experiments dealt with relatively simple characters. The scope of work on inheritance broadened out in due time, and more complex cases were studied, solved, and interpreted in accordance with the theory of alternative inheritance. From time to time complete lists of the various Mendelizing characters have been published, showing the wide range of applicability of Mendel's law. Numerous experiments indicate that the factors for a pair of allelomorphic characters segregate from each other in gametogenesis and recombine in fertilization according to the laws of probability. This hypothesis is accepted on the evidence of the behavior of visible characters in crosses, for segregation of a dominant factor from its recessive mate would give in certain crosses a distribution of somatic characters in classes according to the formula $(3+1)^{n}$ (where $\mathrm{n}$ equals the number of allelomorphic pairs). Actual results agree with this theoretical interpretation. Many characters, however, do not lend themselves to such a simple solution. The inheritance of many size-characters is a matter of much contention. Some maintain that a cross between two individuals differing in size or in a particular size-character may result in a real blend. Others assert that the inheritance of size-characters is essentially Mendelian, or is susceptible of such an interpretation. It can, by no means, be considered that the question of size-inheritance is settled.

In the case of Mendel's peas, tallness and dwarfness were found to be an allelomorphic pair. Other similar cases in plants are well known. The abnormal shortness of bones and general stature in cases of brachydactylism (Farabee 1905, Drinkwater 1908) is inherited alternatively. In order to study size inheritance advantageously, it is quite necessary to have two parent races which breed true to their particular sizecharacter. The absolute difference between the parental characters should be large enough to admit of no confusion. The range of variability for each character should be such that they do not overlap. Environmental influences should not obliterate the difference between the races. The coefficient of variability for each parent race should be small. However, animals which are adapted to genetic experimentation have met in very few cases these essential conditions. Therefore 
the inheritance of their size characters or general body size must be interpreted cautiously. Most cases of size-inheritance in both plants and animals are complex and require a special interpretation, which is, naturally enough at this period, Mendelian in nature.

Throughout a number of recent papers on size-inheritance, there has been, in the main, one mode of explanation. Briefly stated, this explanation hypothecates a number of size-determining factors, the accumulative effect of which adds increments of size to the recessive small type. It is assumed that no one of the factors is completely dominant. In other words, size is thought to be due to multiple factors with incomplete dominance. Such a hypothesis must be carefully distinguished from the cases involving multiple factors for characters with complete dominance. To make the distinction clear, let us make use of a hypothetical case involving multiple factors for one character, such that one parent is homozygous for two factors, $A_{1}$ and $A_{2}$, while the other parent lacks both of these. Using the ordinary Mendelian notation, the cross is as follows:

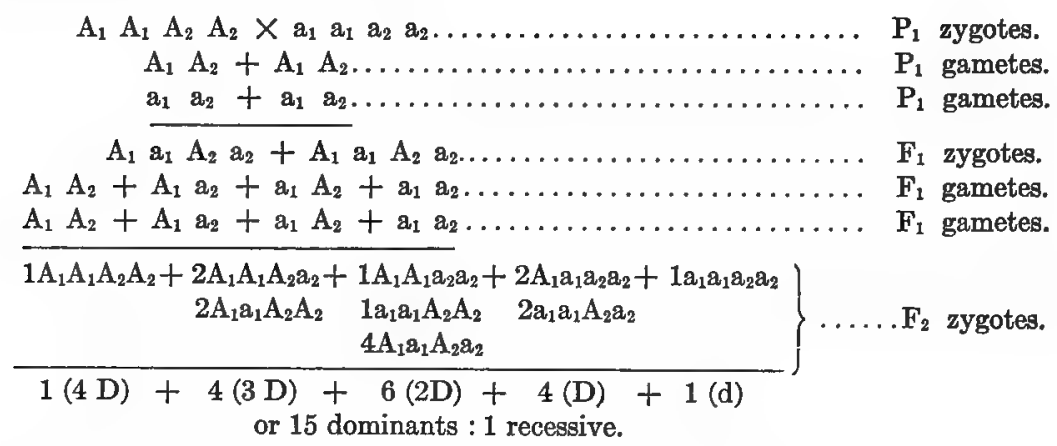

Now if, on the one hand, this illustrates a case involving two factors for a character, with complete dominance, then the $\mathrm{F}_{1}$ generation appears like the dominant parent, and the $\mathrm{F}_{2}$ zygotes consist of 15 dominants : 1 recessive. The first four classes in the $\mathrm{F}_{2}$ generation contain from one to four doses of a dominant factor for the character; hence with complete dominance they are like the $F_{1}$ generation and the dominant parent. The heterozygous condition of such completely dominant factors can not be distinguished from the homozygous. The $F_{2}$ generation has split up into $4^{\mathrm{n}}-1$ dominants : 1 recessive, $\mathrm{n}$ being equal to the number of allelomorphic pairs. The class $\mathrm{A}_{1} \mathrm{~A}_{1} \mathrm{~A}_{2} \mathrm{~A}_{2}$ has altogether four doses of a dominant factor, but since one dose of these factors is completely dominant, it is put in the same visible class as $A_{1} a_{1} a_{2} a_{2}$ or $a_{1} a_{1} A_{2} a_{2}$. The $F_{3}$ generation should demonstrate the existence of the different kinds of $\mathrm{F}_{2}$ zygotes, which from external appearance are grouped together as 15 dominants. Actual cases of this kind have been demonstrated by Nillson-Ehle $(1909,1911)$, and East and Hayes (1911). 
But, on the other hand, if the two factors $A_{1}$ and $A_{2}$ in this same cross were incompletely dominant, the $\mathrm{F}_{1}$ generation would be intermediate and the $\mathrm{F}_{2}$ generation would present a normal curve. Bearing in mind the cumulative effect of each added dose of a dominant factor, according to this hypothesis, and that the visible effect of a factor in the heterozygous condition is half that of a homozygous condition, there should be a graded series in the $F_{2}$ generation ranging from individuals with the cumulative effect of four doses of the dominant factors $A_{1}$ or $A_{2}$ to individuals without either $A_{1}$ or $A_{2}$. For example, if one dose of either $A_{1}$ or $A_{2}$ was responsible for increments of height to the extent of 1 inch, the dominant was 12 inches tall, and the recessive parent was 8 inches tall, then the $F_{1}$ class would be intermediate or 10 inches tall; for, there would be two increments of an inch each added to the recessive type due to the presence of $A_{1}$ and $A_{2}$ in single dose. The $F_{2}$ classes, however, would consist of an array due to segregation and recombination of factors, this array being like a symmetrical curve. There would be:

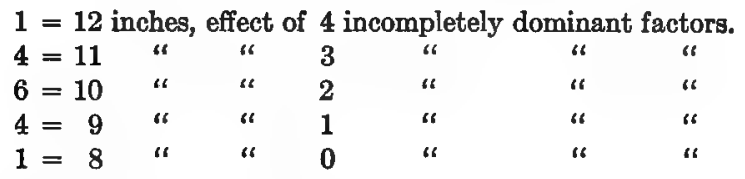

The formula for such an $\mathrm{F}_{2}$ distribution is obviously not $4^{\mathrm{n}}-1$ dominants: 1 recessive, as in the previous case involving complete dominance. With incomplete dominance, the numerical distribution of $\mathrm{F}_{2}$ classes followed the expansion of the binomial $(1+1)^{4}, i_{\text {. e. }},(1+1)^{2 \mathrm{n}}$, where $\mathrm{n}$ equals the number of allelomorphic pairs. The smallest total number of individuals necessary for a complete representation of all $F_{2}$ combinations in their proper proportions is 16 , or $4^{\mathrm{n}}$, the sum of the series $(1+1)^{2 n}$. In dealing with crosses, such as the one just used, in which the size-characters are theoretically due to multiple factors without complete dominance, we should obtain numerical distributions of classes in the $\mathrm{F}_{2}$ generation, together with the number of times an incompletely dominant factor (D) is represented, as follows:

\begin{tabular}{|c|c|c|}
\hline $\begin{array}{c}\text { Allelo- } \\
\text { morphic } \\
\text { pairs. }\end{array}$ & Distribution of $F_{2}$ classes. & $\begin{array}{l}\text { Total } \\
\text { of indi- } \\
\text { viduals. }\end{array}$ \\
\hline 1 & 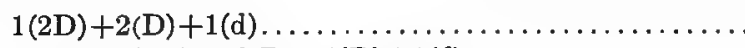 & 4 \\
\hline 2 & $1(4 D)+4(3 D)+6(2 D)+4(D)+1(d) \ldots \ldots \ldots \ldots \ldots \ldots$ & 16 \\
\hline 3 & $1(6 \mathrm{D})+6(5 \mathrm{D})+15(4 \mathrm{D})+20(3 \mathrm{D})+15(2 \mathrm{D})+6(\mathrm{D})+1(\mathrm{~d}) \ldots$ & 64 \\
\hline 4 & $\begin{array}{l}1(8 \mathrm{D})+8(7 \mathrm{D})+28(6 \mathrm{D})+56(5 \mathrm{D})+70(4 \mathrm{D})+56(3 \mathrm{D})+ \\
28(2 \mathrm{D})+8(\mathrm{D})+1(\mathrm{~d}) \ldots \ldots \ldots \ldots \ldots \ldots\end{array}$ & 256 \\
\hline $\mathbf{n}$ & 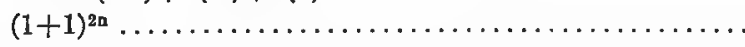 & $4^{n}$ \\
\hline
\end{tabular}


To cover the general case involving any number of such multiple factors with incomplete dominance, we may say that with " $n$ " allelomorphic pairs we theoretically obtain in a total of $4^{\mathrm{n}}$ individuals a series of classes with coefficients derived from the expansion of the binomial $(1+1)^{2 \mathrm{n}}, i . e$. , the series:

$$
\begin{gathered}
1(2 n D)+2 n[(2 n-1) D]+\frac{2 n(2 n-1)}{1.2}[(2 n-2) D]+\frac{2 n(2 n-1)(2 n-2)}{1.2 .3}[(2 n-3) D] \\
+\ldots+2 n[\{2 n-(2 n-1)\} D]+1(2 n-2 n) D .
\end{gathered}
$$

The character of each class is shown by the number of times it contains a dominant factor, $\mathrm{D}$. This is in the form of an arithmetical progression, the first term being $2 \mathrm{nD}$ and each succeeding term being smaller by $\mathrm{D}$, until the last term becomes $2 \mathrm{nD}-2 \mathrm{nD}$, meaning that the ultimate recessive contains no dominant factors whatsoever. In other words, the progression is the same as the exponents of the first term of our expanded binomial $(1+1)^{2 \mathrm{n}}$.

When the $\mathrm{F}_{2}$ generation is produced from the $\mathrm{F}_{1}$, not by mating $\mathrm{F}_{1}$ individuals inter se, as above, but by mating these $\mathrm{F}_{1}$ individuals to either the larger or smaller parent, then the formula $(1+1)^{2 \mathrm{n}}$, does not fit the distribution of classes in the $F_{2}$. The expression $(1+1)^{n}$ is used instead. This can be easily seen by taking a hypothetical case, in which a larger race homozygous in three dominant size factors and having a zygotic formula AABBCC is crossed with a smaller race lacking these, and having a formula aabbcc. The heterozygous $F_{1}$ generation would have a formula $\mathrm{AaBbCc}$. In crossing these $F_{1}$ hybrids back to the smaller parent, we get a distribution of classes as follows:

$\mathrm{ABC}+\mathrm{AbC}+\mathrm{ABc}+\mathrm{Abc}+\mathrm{aBC}+\mathrm{aBc}+\mathrm{abC}+\mathrm{abc} \ldots \quad \mathbf{F}_{1}$ gametes.

abc + abc................................ smaller parent gametes.

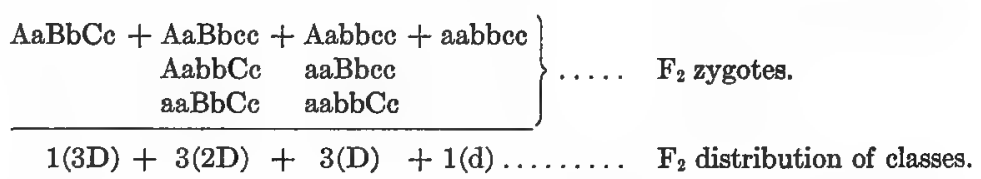

It is apparent that with three allelomorphic pairs the coefficients of the classes are derived from the expansion of $(1+1)^{3}$, and that each class has the dominant-size factor represented one less time than the preceding class, the first class having it three times. The total number of individuals is $2^{3}$ or 8 . Hence, for " $n$ " allelomorphic pairs we would theoretically expect a series as follows:

$$
\begin{gathered}
1(n D)+n[(n-1) D]+\frac{n(n-1)}{1.2}[(n-2) D]+\frac{n(n-1)(n-2)}{1.2 .3}[(n-3) D] \\
+\ldots+n[\{n-(n-1)\} D]+1(n-n) D
\end{gathered}
$$

This means that the coefficients for the classes are derived from the expansion of $(1+1)^{\mathrm{n}}$ and the dominant factors are represented in the 
elasses in an arithmetical progression derived from the exponents of the first term of the binomial, i. e., $n, n-1, n-2, n-3, \quad$. . . $n-n$. The total individuals in the series would be $2^{\mathrm{n}}$.

Had the same heterozygous $F_{1}$ hybrids been mated to the larger parent instead of the smaller, the distribution of classes in the resulting $\mathrm{F}_{2}$ generation would appear as follows:

$$
\begin{aligned}
& \mathrm{ABC}+\mathrm{AbC}+\mathrm{ABc}+\mathrm{Abc}+\mathrm{aBC}+\mathrm{aBc}+\mathrm{abC}+\mathrm{abc} \ldots \ldots \mathrm{F}_{1} \text { gametes. }
\end{aligned}
$$

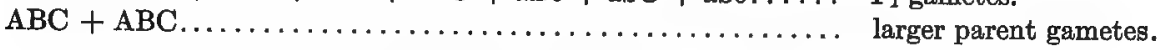

$$
\begin{aligned}
& \begin{array}{c}
\mathrm{AABBCC}+\begin{array}{c}
\mathrm{AABbCC}+\mathrm{AABbCc}+\mathrm{AaBbCc} \\
\mathrm{AABBCc} \quad \mathrm{AaBBCc} \\
\mathrm{AaBBC} \quad \mathrm{AaBbCC}
\end{array} \\
\hline
\end{array} \\
& 1(6 \mathrm{D})+3(5 \mathrm{D})+3(4 \mathrm{D})+1(3 \mathrm{D}) \ldots \mathrm{F}_{2} \text { distribution of classes }
\end{aligned}
$$

It is apparent here that the coefficients of the classes are again derived from the expansion of $(1+1)^{3}$, but, unlike the previous illustration, we find the dominant factor represented in the classes in an arithmetical progression, the first term of which is equal to twice the number of allelomorphic pairs. Hence, for " $n$ " allelomorphic pairs we would theoretically derive a series as follows:

$$
\begin{gathered}
\left.1(2 n D)+n[(2 n-1) D]+\frac{n(n-1)}{1.2} \Gamma(2 n-2) D\right]+\frac{n(n-1)(n-2)}{1.2 .3}[(2 n-3) D] \\
+\ldots+n[\{2 n-(n-1)\} D]+1(2 n-n) D .
\end{gathered}
$$

The gist of all this is that the $\mathrm{F}_{2}$ generations of which we are speaking would theoretically show a range from the larger to the smaller parent with the mode in center when the $\mathrm{F}_{2}$ has been produced by mating the $\mathrm{F}_{1}$ individuals inter se. An $\mathrm{F}_{2}$ generation produced by mating the $\mathrm{F}_{1}$ to the smaller parent shows a range from the $F_{1}$ to the smaller parent, with the mode half way between these. An $\mathrm{F}_{2}$ generation produced by mating the $F_{1}$ to the larger parent shows a range from the $F_{1}$ to the larger parent with the mode half way between.

This is, briefly, the theory of multiple factors as applied to sizeinheritance. If, after sufficiently numerous experiments with plants and animals, it is found to be applicable to such complex cases, it will show that segregation into apparently continuous classes is really discontinuous, or, in other words, Mendelian.

At present we know of no adequate hypothesis, other than the Mendelian, by which to explain the uniform $F_{1}$ generation, the more variable $\mathrm{F}_{2}$ generation, the recovery of parental types, and the tendency for certain recombinations to breed true while others split up again. There is a small number of cases of size-inheritance in which a Mendelian explanation seems well justified. It is logically defensible to resort to this explanation when possible, since it fits a large number of cases involving qualitative characters. However, it is too early to insist that size-inheritance is universally Mendelian, for the number of crucial experiments is few. 
In actual breeding experiments one would undoubtedly meet with much deviation from a perfect blend of quantitative characters in the $F_{1}$ generation, or from such a distribution of $F_{2}$ classes according to the formula $(1+1)^{2 n}$, as in the hypothetical case used above as an illustration. This is particularly true of size-characters in which the theory of multiple factors, incompletely dominant, is most often invoked; for external conditions affect growth and size very easily. Furthermore, there are many other misleading circumstances in such a complex that render analysis difficult. How often could we be sure that a parent race possessed, or was homozygous in, each one of the multiple factors affecting a character; or how often would we find them so, especially in animals? Different individuals in the parent strains might appear alike in a certain character and yet carry different sets of genes for this character. Hayes (1912) had a case in tobacco which could be interpreted in this way. He crossed two varieties of Nicotiana tabacum, both having about the same mode, mean, and low coefficient of variability with regard to number of leaves. The $F_{1}$ was like the parents, but the $\mathrm{F}_{2}$ showed such a marked increase in variability that he was led to believe there had been a recombination of several factors for leaf-number. The argument involved in his explanation is essentially as follows: one parent might have a formula $\mathrm{AABBccdd}$ and the other parent aabbCCDD. They would be of the same leaf-number, since each had the cumulative effect of a double dose of two factors, and they would breed true because each was homozygous. The $F_{1}$ generation, $\mathrm{AaBbCcDd}$, would also be of the same leaf-number, having the cumulative effect of four factors. But when the $F_{1}$ plants were crossed, the $\mathrm{F}_{2}$ generation could have recombinations ranging from AABBCCDD to aabbccdd. The frequency distribution of the classes would be obtained by expanding the binomial $(1+1)^{8}$. Hence, plants occurred with much larger and with much smaller leaf-numbers than in the parental forms or the $F_{1}$ generation. Thus, in actual breeding experiments, one might use parent plants which were of identical appearance but of different zygotic formulæ.

In the simple illustrations of the theory, we suppose that one dose of each factor, such as $A_{1}$, lends an effect about equal to that of any other factor, such as $A_{2}, A_{3}, A_{4} \ldots A_{n}$. But we do not really know for how much influence each factor might be responsible, or whether any one factor always causes the same result under all conditions. Factors in a heterozygous condition may act more vigorously (East and Hayes 1912), or the vigor due to heterozygosis might raise the size of certain classes only. Sterility or partial sterility of one sex might also impair any sort of an analysis on the theoretical scheme suggested.

Environmental influence might affect certain individuals subject by chance, or they might regularly affect individuals of a particular zygotic formula. 
Physiological correlation is not always explained by gametic coupling. It is not difficult to understand how a whole organism or parts of an organism are permanently influenced by even normal conditions. For example, we should hardly expect a normal but very small rabbit to have as large ears as a large rabbit, although both might have theoretically the same set of genes for ear-size. In fact, if one carried out the whole scheme of independent size-factors without reference to physiological correlation it would lead to an absurdity. If a guineapig had genes for a small radius and a large ulna, or for a large tibia and a small fibula, would the animal be a cripple? In dealing with the inheritance of size of certain bones of the body, one can not overlook the influence which other parts, or even the whole body itself, may have upon the development of particular characters studied, irrespective of the hypothetical genes.

It is well known that certain color characters in plants and animals develop only through the interaction of two or more independently transmitted factors. Thus, the factor for the agouti pattern in the hair of rodents acts only when black or brown is present in the zygote; but black or brown pigments in turn are restricted to the eyes and extremities unless the extension factor is present. It may be added that the basic color factor must also be present in order to activate the development of color. Therefore, to obtain the agouti pattern it is necessary to have at least four independently heritable factors, viz, the color factor, the extension factor, the brown or black factors, and the agouti factor. When we recall such facts as these, and realize that several or many factors may interact in the production of sizecharacters, we see how difficult it is to attempt or rather attain a satisfactory solution.

Considering briefly the evidence which tends to show that a number of factors may exist for one and the same visible character, we find comparatively few experiments. Most of these are in plants. NillsonEhle $(1909,1911)$ paved the way by showing how some apparently continuous variations might be interpreted as discontinuous variations. The black glumes of oats, he showed, might be due to two factors, either of which alone could cause the development of black in the glume. If a plant homozygous for both kinds of black $\left(\mathrm{B}_{1} \mathrm{~B}_{1} \mathrm{~B}_{2} \mathrm{~B}_{2}\right)$ was crossed with a plant lacking black $\left(b_{1} b_{1} b_{2} b_{2}\right)$, the heterozygotes were black and hold a formula $\mathrm{B}_{1} \mathrm{~b}_{1} \mathrm{~B}_{2} \mathrm{~b}_{2}$. Crossing the heterozygotes inter se gave an average of one entirely recessive individual in every 16 . It proved to be a simple dihybrid cross, in which 15 of every $16 \mathrm{~F}_{2}$ individuals carried at least one dose of a dominant factor and were black. The $\mathrm{F}_{2}$ generation should theoretically consist of $9 \mathrm{~B}_{1} \mathrm{~B}_{2}: 3 \mathrm{~B}_{1} \mathrm{~b}_{2}: 3 \mathrm{~b}_{1} \mathrm{~B}_{2}$ : $1 b_{1} b_{2}$. Since either factor $B_{1}$ or $B_{2}$ caused a development of black in the glume, the first three classes were alike black. Subsequent 
self-fertilization proved that the $F_{2}$ individuals were of the formula demanded by such an explanation, viz:

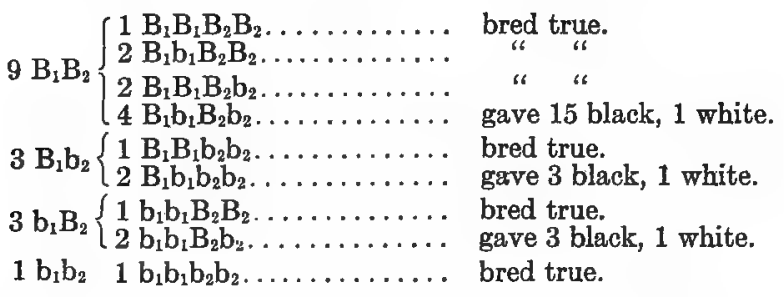

$B_{1}$ was not allelomorphic to $B_{2}$, but each was allelomorphic to its own absence; both $B_{1}$ and $B_{2}$ caused development of black in the glume independently.

Carrying out similar work on other characters, Nillson-Ehle found that the presence of red in the pericarp, presence of brown in the ears, presence of ligules, internodal length, rust resistance, and the like were due to more "present mutually independent, separable factors than might be concluded from external appearances." In any such case involving $\mathrm{n}$ allelomorphic pairs, the ultimate recessive would appear in 1 out of $4^{\mathrm{n}}$ individuals. In a trihybrid or tetrahybrid cross, the ratios would be $63: 1$ and $255: 1$ respectively-subject, of course, to the law of error. It is true that the dominant classes may often show whether they contain a smaller or larger quota of the dominant factors. Environmental conditions may also prevent the complete somatic development of the characters which a plant may transmit to its progeny.

Emerson (1910) gave a short, concise interpretation, in Mendelian terms, of the inheritance of shape and size in three species of plants. His data (on size and shape of the fruits in gourds and summer squashes, size and shape of bean seeds, and size of seeds and height of the stalk in corn) show a blend in the $F_{1}$ generation and a marked increase of variability in the $\mathrm{F}_{2}$ generation over the parents or $\mathrm{F}_{1}$ generation. The difference between the $\mathrm{F}_{1}$ and $\mathrm{F}_{2}$ plants is great enough to leave no doubt that this increased variability has been delayed until the second generation after the cross. Shull (1910) reports a similar increase in the $\mathrm{F}_{2}$ generation in the variability of the number of rows per ear in corn.

East and Hayes (1911) likewise demonstrated that yellow in the endosperm of maize may be due to two factors, $\mathrm{Y}_{1}$ and $\mathrm{Y}_{2}$, each allelomorphic to its own absence. Hence, they obtained in a cross between a homozygous yellow race $\left(\mathrm{Y}_{1} \mathrm{Y}_{1} \mathrm{Y}_{2} \mathrm{Y}_{2}\right)$ with a white race $\left(\mathrm{y}_{1} \mathrm{y}_{1} \mathrm{y}_{2} \mathrm{y}_{2}\right)$ a ratio of 15 yellows: 1 white. In crossing types of maize, differential characters in the number of rows per ear, length of plant, length of ear, and weight of seed were studied. By crossing the dominant with the recessive type of each character, an increased coefficient of variability 
was obtained in the $F_{2}$ generation. This they held due to a rearrangement of a number of separable factors for the character involved.

Tammes (1911) has likewise thought it possible to ascertain a number of separate, independent factors for characters in species and varieties of flax (Linum). She has calculated the approximate number of factors for each character, sueh as length and breadth of the seeds, length and breadth of the petals, color of the flowers, and dehiscence of the capsules. The proportionate number of individuals in the $\mathrm{F}_{2}$ generation, which show the pure parental character, was taken as an index of the number of factors for that character.

Phillips (1912) has recently crossed two races of ducks, differing in size, and obtained an increase in variability in the $\mathrm{F}_{2}$ generation. MacDowell (unpublished) had similar experience with rabbits. An increase in variability in the $\mathrm{F}_{2}$ generation can not in itself be considered a final criterion of Mendelizing inheritance, for the $F_{2}$ individuals should be tested in order to show that all do not regress to the mean, but some pure recombinations have been formed. Very little has been done with $F_{3}$ generations in such crosses.

East and Emerson (1913) have continued their researches in maize on the inheritance of number of rows per ear, length of ears, diameter of ears, weight of seeds, breadth of seeds, height of plants, number of nodes per stalk, internodal length, number of stalks per plant, total length of stalks per plant, and duration of growth, and have given evidence that the $\mathrm{F}_{2}$ generation is in general more variable than the $F_{1}$ or either parent. Furthermore, the $F_{3}$ generations indicated that the parental types recovered in the $F_{2}$ might breed true, that intermediate types with new modes had been obtained, and that some $\mathrm{F}_{2}$ individuals gave $F_{3}$ progeny just as variable as the $F_{2}$. They conclude "that the results secured in the experiments with maize were what might well be expected if quantitative differences were due to numerous factors inherited in a strictly Mendelian manner."

The striking similarity between these crosses and some of the wellknown color crosses makes it seem probable that both forms of inheritance may be Mendelian; for in both the segregation is delayed until the $\mathrm{F}_{2}$ generation. Nevertheless, the clearness shown in color-inheritance does not stand out in size-inheritance. Interaction of many factors and environmental effects may play a greater part. Whether or not the general size of mammals will lend itself to such a solution is difficult to say. There is much correlation in the size of parts, although we do find that partially uncorrelated individual parts, such as short legs, tails, or ears, may exist in mammals. It would be theoretically and practically desirable to know whether the inheritance of the general body size is Mendelian when mammals of the same proportions but of different size are crossed. 
The two parent species, C. porcellus and C. rufescens, and their hybrids of various blood dilutions, which formed the material for Part I, are also used as the basis for Part II. Each parent species is of very distinct and specific size, such that environment does not obliterate the difference. Unfortunately, the cross involved sterility and necessitated crossing back to males of the parent species. A careful examination of growth curves and skeletal dimensions was made to study sizeinheritance in such a mammalian species cross and to compare it with the work already cited.

\section{GROWTH.}

THE DATA.

Cavia rufescens is a smaller species than Cavia porcellus. The average healthy adult weighed about 425 grams; the females were a trifle lighter, or about 420 grams. One male ( $\left.o^{x} 1\right)$ alone reached the 500-gram mark in any of his weights; but he was fat, and his weight was above normal. His son, though slightly larger in skeletal dimensions and in good condition, was about the average weight. The average weight of guinea-pigs is twice that of the wild; and since the average is so much larger, it follows that many guinea-pigs are more than twice as heavy. I have never seen a guinea-pig of either sex, with a normal healthy growth curve, maintain such a low weight as the wild. This statement is made on the basis of an intimate acquaintance with the growth curves of several hundred guinea-pigs. In order to study the growth curves of the parent species and hybrids, the weights were taken about once a week until the curve was well established. After that, observations were made at less frequent intervals. The weights of pregnant females were taken during the period of gestation, but not used on account of the varying number of fetuses. Having obtained the weights, the growth curve of each animal was plotted on coordinate charts by placing the days on the abscissas and the grams on the ordinates.

Any individual curve would naturally show a depression when external conditions were poor and an elevation when conditions were conducive to fatness. Since no growth curve is in itself an infallible expression of the general growth tendencies of an animal, a second set of curves was drawn, in which the irregularities were smoothed to show as nearly as possible the normal growth of each individual. This may seem arbitrary; but in reality it does not signify any bias, for in all cases the smoothed curve was determined by the majority of points in the actual curve. Minot (1891) has shown "that any irregularity in the growth of an individual tends to be followed by an opposite, compensating irregularity; and that variability decreases with age." To be concrete, all animals in these experiments showed a decrease 
in weight about the end of April, when the quality of beets and turnips was poor and the supply was low; but toward the middle of May they recovered completely through copious feedings with fresh green grass and winter rye. The irregularities in growth were caused by external conditions, and observations of these conditions were made. It requires no stretch of the imagination or undue speculation to smooth such irregularities and thus procure a curve which more truly shows the general growth tendency in an animal. Smoothed curves were made for parent and hybrid individuals as follows:

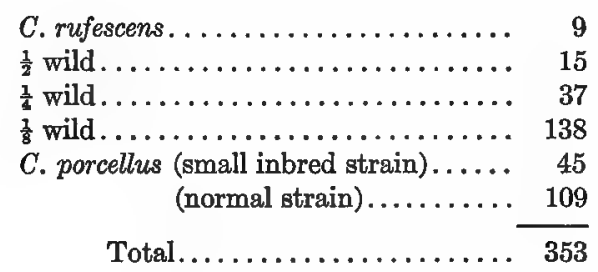

All sick animals and those whose curves were not well established because of early death or present immaturity, were neglected. Diseased animals show an irregular curve with a large final loss in weight and wore therefore neglected. Many other guinea-pigs and hybrids have been studied, and can be added, when their growth is sufficiently complete. It is quite significant that a duplicate set of smoothed curves was made for about 75 animals. This set did not vary much from the first set. We thus have a check on errors in judgment, for the duplicate set was made a number of months after the first set and without any reference to the same. I am, therefore, led to believe that the average of the smoothed curves is correct within $=25$ grams.

Having once obtained the smoothed curves, composite curves for the males and females of different classes were calculated. The method is simply to find the arithmetic average of the weights in the smoothed curves at 15 different intervals (tables 59 and 60). For example, if we average the weights of all $\frac{1}{8}$ wild hybrid males at the age of 100 days, as given in their smoothed curves, we obtain an average of 555 grams. This gives one point from which to plot the average of the curves of animals in that group. The other points were similarly calculated, and a composite curve or average of the smoothed curves was plotted. The composite curves of the wild and tame species and three classes of hybrids are shown in text-figures 1 and 2.

Needless to say, all animals were kept in healthful quarters, with an abundant supply of food and water. The food at all times consisted of oats. In the winter this was supplemented by daily feedings of beets or turnips; in the summer, by fresh green grass and clover. 


\section{COMPARISON OF GROWTH CURVES.}

The Averages.

Minot (1891) has shown, in the case of the guinea-pig, that growth is rapid at first; and as the animal grows older a smaller daily increment is added. Stating it differently - as an animal grows older it requires a constantly increasing span of time to add successive, equal increments of weight, until finally growth ceases. This means that the growth curve is steep at first, and that the early growth is the greatest. Gradually the curve approaches a straight line, the adult weight. The composite curves for both sexes (text-figures 1 and 2) show this in the wild, the tame, and the hybrids.

At the end of a year practically all of the animals were full-sized adults; but in nearly all cases an extra 3 months was given to each animal to follow a full compensation for any possible early retard. At the end of a number of curves a slight unexpected increase appears. This is due to the fat condition of a number of the older animals, as the individual records show. One can easily follow any curve to its logical conclusion.

From an examination of tables 59 and 60 and their graphic representation in text-figures 1 and 2, a number of salient facts, concerning the average weights of the parent species and the hybrids, may be recorded:

(1) The average weights, and consequently the composite growth curves of the wild, are well below the tame guinea-pig at all ages and in both sexes. These do not show, however, that this is not completely true for all individual weights of each species. For example, the individual records reveal that some male guinea-pigs at the age of 10 days were lighter than 95 grams. Although there was some overlapping of the early individual weights of the wild and tame, as time progressed the wild showed their specific character, and it required only a few weeks before all the wild were well below all the tame. Weights were obtained for more than 4 wild males and 5 wild females. Originally, composite curves were made including these. They only served to augment the difference between the wild and the tame, for they were sickly, did not thrive well in captivity, and died prematurely. Those animals which entered into the tables and composite curves represented in a fair way the natural growth of the wild Cavia rufescens.

(2) The $\frac{1}{2}$ wild hybrids of both sexes were remarkably vigorous animals. The males attained an average which exceeded their larger parent, the guinea-pig. They were also larger than all succeeding hybrids. The females were likewise very vigorous. Curiously enough, the middle portion of the composite curve of the females is below the guinea-pig and the $\frac{1}{8}$ wild. But, if an anticipation is permitted, it will be shown that the bones of the $\frac{1}{2}$ wild hybrids are larger than those 


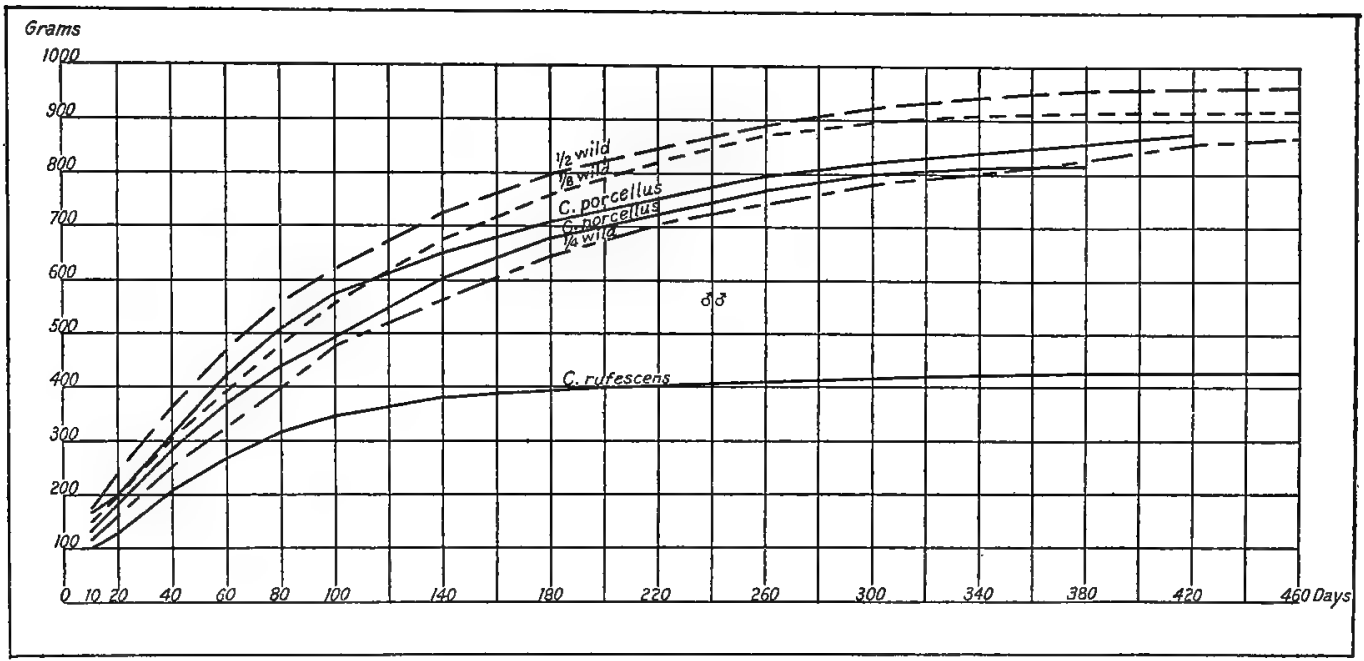

TeXT-FIgurs 1.-Composite growth curves of the males in the parent species and hybrids.

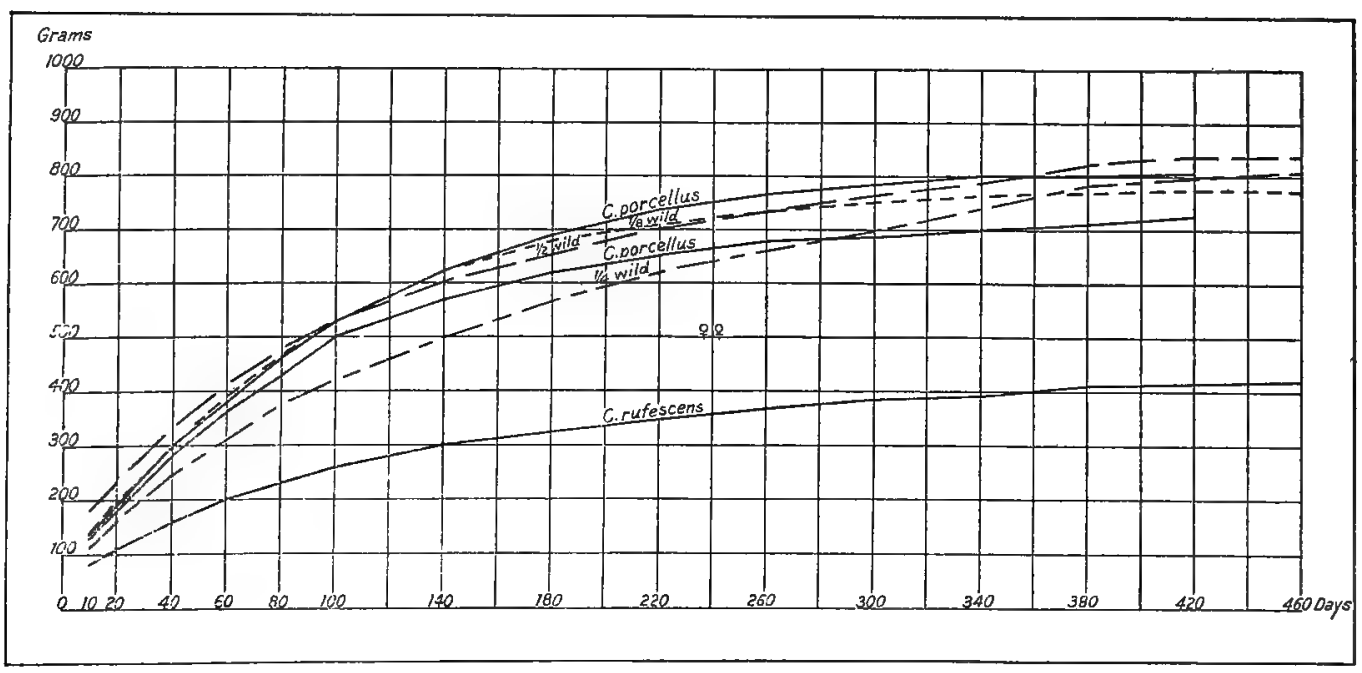

Text-FIgURn 2.-Composite growth curves of the females in the parent species and hybrids. 
of the guinea-pig or of other classes of hybrids. The depression in the composite curve of the $\frac{1}{2}$ wild females from the 120th day to the 340 th day was due largely to our eager haste to breed these unusual hybrids as soon and as frequently as possible. Furthermore, I should not consider the composite curve as trustworthy as the skeletal dimensions; because adult weights are more variable than adult skeletal dimensions; and because possible errors in judgment arise, especially when one subconsciously tries to avoid a bias in favor of "too much heterozygosis" in smoothing the individual growth curves from which the composite curves were calculated.

The species cross between the horse and ass gives the well-known vigor for which the mule is so highly valued. Darwin (1876) pointed out that cross-bred plants were often more vigorous than the inbred parents. East and Hayes (1912) have concluded that the vigor is in a measure proportional to the number of factors in a heterozygous condition. Our $\frac{1}{2}$ wild hybrids were undoubtedly heterozygous in many factors, but we can not be sure that the more vigorous were heterozygous in a greater number of factors. What part sterility may play is also unknown.

(3) The $\frac{1}{4}$ wild of both sexes clearly lacked the vigor which characterized the $\frac{1}{2}$ wild. The composite curves of the males and females lie entirely below those of the $\frac{1}{2}$ wild. The greater part of both also lies below the guinea-pig and the $\frac{1}{8}$ wild. Although these $\frac{1}{4}$ wild were produced by mating the vigorous $\frac{1}{2}$ wild back to the larger of the original two parent species, it is obvious that both the males and females were smaller at all ages than the $\frac{1}{2}$ wild, and also smaller than the guinea-pig during the larger part of their growth curve.

If we regard the sexes separately, it will be seen that the $\frac{1}{4}$ wild males averaged less than the guinea-pig throughout the greater part of their growth curve, for they lie distinctly below these up to the age of 360 days. Their curves take an unexpected rise at the age of 340 days, but from personal experience with these animals $I$ am led to believe that this was due to the obesity of a number of older males which were kept alone to prevent fighting. The difference between the $\frac{1}{4}$ wild males and the $\frac{1}{2}$ wild is quite apparent, for they are separated by an average of about 150 grams during a large part of their growth. It is difficult to ascertain how much significance to attach to the average difference between the $\frac{1}{4}$ wild males and their smaller parent, the guinea-pig. They were consistently lower at all ages than the smaller race of guinea-pig males until 360 days, although the difference was not great.

The $\frac{1}{4}$ wild females resembled their brothers in many respects. They likewise lie below the guinea-pig during the greater part of the growth curve, for they were smaller up to the age of 260 days. Their growth 
curve rises above the smaller race of guinea-pigs at this date, and this is not due to an abrupt change in their curve, as was the case with their brothers. Like their brothers, they averaged less than their $\frac{1}{2}$ wild parent at all ages and the difference is also well defined.

Summarizing, we may say that the $\frac{1}{4}$ wild of both sexes lacked the vigor of the $\frac{1}{2}$ wild, although the $\frac{1}{2}$ wild females were used as one parent. The $\frac{1}{4}$ wild males were in general smaller than the guinea-pig parent; but the $\frac{1}{4}$ wild females did not agree perfectly with their brothers, for they did not average less than the guinea-pig as constantly.

(4) The $\frac{1}{8}$ wild showed a complete return to the parental guinea-pig average and any possible indication of the loss of vigor shown by the $\frac{1}{4}$ wild parent was absent. The $\frac{1}{8}$ wild males have a composite growth curve which is actually higher than the larger guinea-pig race after the 140th day. The $\frac{1}{8}$ wild females agree closely with the larger guineapig race. It is possible that the composite curve of this hybrid class of males is higher than it should be, for on account of sterility they were unmated and often kept alone to prevent fighting. On the whole, we may consider the $\frac{1}{8}$ wild of both sexes the equal of the larger race of guinea-pigs. The $\frac{1}{8}$ wild males averaged larger than the $\frac{1}{4}$ wild males throughout their whole life. Their sisters were larger than the $\frac{1}{4}$ wild females up to the age of 340 days, or, in other words, until about that time when the adult size was reached. The $\frac{1}{8}$ wild, however, did not equal the vigor of the $\frac{1}{2}$ wild. The data on skeletal dimensions will corroborate all these facts in a general way.

(5) Two composite curves are given for each sex in the case of the guinea-pig. One curve represents the average growth curve of a healthy, vigorous strain of guinea-pigs. The other curve is taken from the records of a closely inbred strain which was not so vigorous; hence the latter lies below the former at all points. The stock used as the guinea-pig parent in these experiments corresponded closely to the larger strain. The difference between the two curves shows the possibilities with the species $C$. porcellus itself.

(6) The average weights of the females, and hence their composite growth curves, were below those of the males at all ages. This was true of both parent species and the three classes of hybrids given. It was equally true of the other classes of hybrids subsequently produced.

Summarizing the general results obtained as shown by all the different averages of weights, it was obvious that (1) the $\frac{1}{2}$ wild were more vigorous than either parent species; (2) the $\frac{1}{4}$ wild lacked this vigor; (3) the $\frac{1}{8}$ wild regained the size of the original larger parent species by the continued crossing back to this species. These facts will be considered later in connection with the discussion of the averages of the skeletal dimensions. 
The Coefficients of Variability.

It is indicated on pages $48-55$ that a number of recent papers on size-inheritance postulated multiple factors for size with incomplete dominance. According to this theory, a cross between a pure large race and a pure small one would result in a blend, in the absence of disturbing influences such as the vigor of heterozygosis, environment, and the like. If the $F_{1}$ generation were then crossed inter se, one should obtain an increased coefficient of variability and, with sufficient numbers, recover the parental forms. If, however, the $F_{1}$ generation were crossed back to either parent, one should obtain a range from the $\mathrm{F}_{1}$ to that parent with the mode in between. The usual method of procedure would be to mate the $F_{1}$ generation inter se in order to obtain a maximum coefficient of variability as the best evidence of segregation and recombination of size factors. But this was impossible in these crosses, for the males were entirely sterile. Two alternatives remained, either to cross the $\mathrm{F}_{1}$ females back to the guinea-pig or to the small wild C. rufescens. The latter would have been preferable, but not enough cases were successful to give data of value, hence all results were based on crossing the $F_{1}$ females back to the guinea-pig. The $\mathrm{F}_{2}$ males were likewise sterile and consequently the $\mathrm{F}_{2}$ females had to be crossed back to the guinea-pig. This meant that conditions made it necessary to resort to the class of matings least advantageous for a study of size-characters.

The study of the average weights at different ages is quite insufficient to show the complete relation between the size of parents and hybrids, for they do not indicate the dispersion of the individuals from the average of the group considered; or, in other words, averages do not give evidence of segregation and recombination of possible unit factors for size. Therefore, the coefficients of variability of the weights of the parents and hybrids were calculated from the individual smoothed curves for six different ages ranging from 100 days to 380 days (see tables 61 and 62 ). It must be stated at the outset that the data and results are very unreliable, for the numbers are small, although breeders of manmals must be content with such; and environment affects growth and weights greatly.

The coefficients of variability for weights of the males and females (tables 61 and 62) give no clear, pronounced evidence that the hybrids of the second generation were more variable than those of the first or than the guinea-pig parent, and hence there is no evidence of segregation and recombination of factors. It is true that some classes of hybrids were very slightly more variable than either original parent species, but it is difficult to know whether this was due to real inherent variability or to experimental error. Furthermore, such differences as do obtain are not wholly consistent with an explanation that postulates 
multiple factors for size with incomplete dominance. For example, the $\frac{1}{2}$ wild males were as variable as the $\frac{1}{4}$ wild males. Had the parent races and the $F_{1}$ hybrids shown a comparatively small degree of variability and the $\mathrm{F}_{2}$ hybrids a decided increase in variability, then we might have concluded that there were indications of a recombination of factors for size. The results by no means disprove that the sizedifference between the guinea-pig and the wild species may not be due to a difference in size factors, but the various crosses actually made failed to give evidence to that effect. One could conclude more logically that (1) the guinea-pig was dominant, or very nearly so, to the wild species in respect to size; (2) the immediate hybrids, the $\frac{1}{2}$ wild, were very vigorous because of heterozygosis; and (3) therefore, repeated crossing back to the dominant form would not increase the variability.

In deciding what the normal growth curve of any individual is, in order to obtain the smoothed curves and calculate the averages and coefficients of variability, errors in judgment may occur. In this particular case the number of individuals was small and experimental errors may have been large; hence no probable errors were calculated for the average weights or coefficients of variability. The adult skeletal dimensions offered material with less objections than did the growth curves. The results of both can be compared.

In passing, it may be pointed out that all classes of individuals in both sexes appeared to be less variable as they grew older.

\section{SKELETAL DIMENSIONS.}

\section{THE DATA ON SKELETAL DIMENSIONS.}

It was shown that the adult weight of $C$. rufescens was much less than that of $C$. porcellus. The bones of the wild are likewise shorter and more slender than those of the tame guinea-pig. In order to make a more extended study of the size relation between the two parent species and their hybrids, measurements of bones were taken from prepared adult skeletons. The materials available were as shown in the accompanying table.

\begin{tabular}{|r|r|r|}
\hline Class. & Male. & Fem. \\
\hline Wild.......... & 3 & 1 \\
$\frac{1}{2}$ wild......... & 5 & 8 \\
$\frac{1}{4}$ wild........ & 16 & 20 \\
$\frac{1}{8}$ wild......... & 60 & 65 \\
Guinea-pig.... & 78 & 63 \\
\cline { 2 - 3 } Total..... & 162 & 157 \\
\hline
\end{tabular}


It was found that the skeletons had completed growth at the end of 15 months. Osseous nodules and ridges, to be sure, are laid down at a later date; but they do not influence the measurements considered. Care was taken to see that sutures between the epiphyses and diaphyses were closed. Furthermore, the suture between the basioccipital bone and the basisphenoid bone is one of the last to fuse in mammalian skulls, and this was completely fused at the age of 15 months. The bone measurements were, therefore, taken from fully adult animals whose bones had reached their maximum size.

In preparing the skeletons all individuals were boiled separately in soap and water. The flesh was brushed away and the bones were dried, properly labeled, and filed in separate boxes. Errors were thus avoided. In all cases the skull, lower jaw, scapula, right front leg, and right hind leg were saved. Whenever possible the entire skeleton of the wild and early hybrids was saved. Unfortunately, a number of adult wild and adult $\frac{1}{2}$ wild were discarded by a laboratory helper when they died.

Sixteen different measurements were taken on all skeletons. In addition to these, 13 more measurements were taken in the case of the wild, the $\frac{1}{2}$ wild, and the $\frac{1}{4}$ wild. The results, given in tables 63 to 66 , were calculated from these measurements. In deciding upon the different possible measurements to be used, those actually used were chosen for the following reasons: (1) They could be taken accurately without any slipping of the calipers; (2) they were the largest measurements, thus diminishing the effect of any experimental errors; (3) they took into account those dimensions in which the wild and tame parents differed in the most marked degree. All the dimensions were taken with sliding vernier calipers and recorded in terms of $0.1 \mathrm{~mm}$. The averages, however, are given in millimeters. For example, the average skull length of 78 male guinea-pigs was $68.48 \mathrm{~mm}$.

The use of skeletal dimensions in a study of size-inheritance has advantages which the weights lack. In the case of the growth curves, two observers might arrive at different conclusions with regard to an adult weight; or even the same observer has slightly different views at different times. The measurements of the adult skeleton, however, were so exact that a remeasurement gave the same result at all times within $\pm 0.2 \mathrm{~mm}$. In repeating many bone measurements, it was found that the second observation tallied completely with the first in almost all cases. When a difference did occur, it was so small as to be negligible. Furthermore, the adult skeletal dimensions were far less variable than the adult weights, meaning that the environment probably affects the weights more. Of course, no claim is made that the adult skeletal dimensions represent the precise genetic possibility of an animal, but under normal conditions they probably approximate it more closely than do the weights. All of these considerations made the skeletal dimensions a better basis for study than weights. 
The measurements considered in the tables are as follows:

Skull measurements:

1. Median sagittal length, from cranial edge of fused premaxillary bones to lambdoidal ridge of occipital bone.

2. From the same cranial edge to the ventrocranial edge of the foramen magnum.

3. Length of the zygomatic arch from the laterocaudal margin of the infraorbital foramen to the caudal margin of the mandibular fossa.

4. From the laterocaudal margin of the infraorbital foramen to the exoccipital bone immediately dorsad of the jugular process.

5. From the premaxillary bone to the medial lachrymal sulcus.

6. From the premaxillary bone to the medial caudal margin of the palatine bone.

7. From the caudal edge of the foramen incisivum to the ventrocranial edge of the foramen magnum.

8. Width immediately craniad of the external acoustic pore.

9. Width at caudal portion of zygomatic arch, where skull is broadest.

10. Width at cranial edge or point of the zygomatic bone.

11. Width at laterocaudal margin of infraorbital foramen.

Mandibular measurements:

12. Extreme length from angular process to laterocaudal margin of incisor alveolus.

13. From concave edge between condyloid process and angular process to cranial edge of first molar alveolus.

Humerus:

14. Length, from fossa between greater and lesser tuberosity to fossa between capitulum and trochlea.

Femur: Tibia:

15. Length, from trochanteric fossa to intercondyloid fossa.

16. Length, from fossa between spine of tibia and lateral tuberosity to lateral concavity at distal end.

COMPARISON OF SKELETAL DIMENSIONS.

The Average Dimensions.

The wild C. rufescens has long been known and recorded by taxonomists as a small cavy species, smaller than the guinea-pig, C. porcellus. Hence, the average skeletal dimensions given in tables 63 and 64 were not taken from individual, small specimens that may have been wide variates. Other wild skeletons were examined and measured, but were omitted for the sake of accuracy in these averages because a few sutures were not closed, although they were sexually mature. They were in reality smaller than the average recorded. A number of fully adult living specimens were carefully examined both in our own laboratory and in European collections and were clearly much smaller than the guinea-pig. The wild, which enter into the averages in tables 63 and 64 , were the two original wild parents used to propagate the wild stock in captivity, and all of their sons ( $\sigma^{7} 24$ and $0^{7} 33$ ) who, with their 
father ( $\left.\sigma^{7} 1\right)$ were used as the wild parent in the crosses that produced the hybrids. They were fully adult, healthy animals, and in all probability as large or larger than most members of their species. The tables indicate that $C$. rufescens is smaller than the tame parent species in all measurements considered. This was also found to be true of the scapula, radius, ulna, innominate bone, fibula, and the different vertebræ. (See figs. 10, 11, 15, 16, 20, 21, 25, 26, 30, 31, and 34 to 41.) The long bones of the wild were likewise more slender than those of the tame. The average skeletal dimensions of the tame were found to be higher than those of the wild in every case in both sexes. It is appropriate to say, briefly, at this point that all the figures of the skulls and bones given in the plates are of natural size and represent as nearly as possible the averages given in tables 63 and 64 . The skulls and bones shown in these plates were chosen because each one represents the average of its class. In all cases the figures are not visibly different from the computed average and any actual difference is generally much less than $1 \mathrm{~mm}$.

It may seem that the differences between the averages of individual measurements are too small to separate the two species distinctly; but if, for example, an average guinea-pig skull is compared with a wild skull (figs. 10,11, 15, and 16), it will be seen readily that the total effect of all these differences in the eleven skull dimensions is enough to separate the wild from the tame distinctly. Furthermore, there is a minimum amount overlapping between individuals of the two species. Although the 2,250 individual measurements for 78 male and 63 female guinea-pigs are not presented, there were very few cases in which any guinea-pig was found to be as small in any of its dimensions as the longest wild. The exact number of guinea-pigs overlapping the wild is as shown in the accompanying table.

\begin{tabular}{|r|r|r|}
\hline $\begin{array}{c}\text { Measure- } \\
\text { ment. }\end{array}$ & Male. & Fem. \\
\hline No. $3 \ldots$ & 0 & 3 \\
$6 \ldots$ & 4 & 0 \\
$8 \ldots$ & 49 & 5 \\
$9 \ldots$ & 5 & 1 \\
$12 \ldots$ & 3 & 0 \\
$15 . .$. & 7 & 0 \\
\hline
\end{tabular}

Therefore, out of a total of 2,250 guinea-pig measurements, there were only 77 which overlapped the corresponding wild measurements. This means that no guinea-pig of either sex was as small as the wild in the case of 10 of the 16 dimensions. In the 6 dimensions given above, there were a few cases in which some guinea-pigs equaled or were smaller than the wild, but when they were smaller it rarely 
amounted to more than $0.3 \mathrm{~mm}$. In measurement 8 , the width of the skull immediately craniad to the external acoustic pore, the males of both species were more nearly equal, and 49 out of 78 male guineapigs were actually as small as or smaller than the largest wild. The reason the wild are so large in this measurement is due to the large bulla, possibly associated with the organs of hearing. Many other guinea-pig skeletons were examined at a later date, but none could be mistaken for the wild species.

The wild C. rufescens in these experiments were, therefore, distinctly smaller than the tame $C$. porcellus. The skeletal dimensions corroborate the data presented in the composite growth curves. The number of wild in tables 63 and 64 is too small to give significant averages; but the known facts regarding $C$. rufescens and our own observations on immature animals indicate clearly that it is specifically smaller than the tame species. Furthermore, since the number of tame is large enough to be significant, it is noteworthy that their lower extremes rarely overlapped with the measurements of our largest, healthy, adult wild animals.

The one-half wild hybrids, obtained by crossing the wild males to guinea-pig females, were larger and more vigorous than either parent species. The males averaged larger in all measurements taken, and the females averaged larger in all but two (see figs. 12, 17, 22, 27, 32 , and 34 to 41 ). In these latter two exceptional cases (measurements 10 and 13) the females were really as large as the guinea-pig, for the difference was hardly significant, considering the probable errors. This increased size and vigor was not only true of the $\frac{1}{2}$ wild as a whole, but every individual male and female was larger than the average guinea-pig in all its measurements, except two $\frac{1}{2}$ wild hybrids. These two exceptions ( $\sigma^{\top} 117$ and $\% 118$ ), a brother and sister, were fully as large as the average guinea-pig. The individual measurements and the averages of the progeny in this first cross thus attested the remarkable vigor of the $\frac{1}{2}$ wild hybrids. The skeletal dimensions, therefore, corroborate the data presented in the composite growth curves. This was not only true of size but also of endurance; for, although they were very wild in disposition and difficult to keep in captivity, when successfully reared they showed their physical strength. They lived through winters when ordinary guinea-pigs succumbed to disease. One female had 15 litters of young and is still breeding at the age of 7 years. Alezais (1903), quoting Metschnikoff, states that this age would be remarkable for a guinea-pig. None of the several thousand guinea-pigs in this laboratory have ever been as long-lived; nevertheless, it must be stated that there has been no close study of their longevity. Other $\frac{1}{2}$ wild females were equally vigorous and fertile, but were killed for the purpose of study. 
We can not dispatch the whole situation by a simple statement that the guinea-pig is dominant in size. Possibly it is somewhat so, but we do not know how much of this vigor and size was due to heterozygosis. Furthermore, since the female was the large parent, it may be that the reciprocal cross, with $C$. rufescens as the female parent, would not have given the hybrids such a good start. It is conceivable that two fetuses in the guinea-pig uterus would have a greater chance for initial development than the same two in the uterus of $C$. rufescens. That the guinea-pig is in all probability not completely dominant one can conclude from the size of the next generation.

The one-quarter wild hybrids were produced by mating the $\frac{1}{2}$ wild females back to guinea-pig males (see figs. 13, 18, 23, 28, 33, and 34 to 41). They showed a striking loss of the vigor which characterized the $\frac{1}{2}$ wild, for both sexes averaged smaller than these in all dimensions, except measurement 3 in table 64 . The single exception was the length of the zygomatic arch in the female sex, in which dimension the $\frac{1}{2}$ wild and $\frac{1}{4}$ wild females averaged exactly the same. The $\frac{1}{4}$ wild not only averaged less than the $\frac{1}{2}$ wild, but no one of the 36 individuals was as large in any measurement as the largest $\frac{1}{2}$ wild, and very few were as large as the smallest $\frac{1}{2}$ wild. Comparing the average of the $\frac{1}{4}$ wild males with their male parent, the guinea-pig, it was found that there was a general tendency for the hybrids to be smaller, in which respect the growth curves and skeletal dimensions again agree. The averages of the male $\frac{1}{4}$ wild were less in all measurements except 8 and 9 . The female $\frac{1}{4}$ wild averaged smaller in all measurements except $3,5,6,8$, and 9. Although the growth curves and skeletal dimensions of the $\frac{1}{4}$ wild were in general consistently lower than those of the guinea-pig. the differences were not great. What seems to be a general tendency must be cautiously considered, in view of the small differences, which were often not much larger than the probable error of the averages.

The one-eighth wild hybrids, or $\mathrm{F}_{3}$ generation, were produced by mating the $\frac{1}{4}$ wild females back to the guinea-pig males (see figs. 14, $19,24,29$, and 34 to 41 ). The males of this generation were larger than the $\frac{1}{4}$ wild in 14 of the 16 dimensions; and the females were larger in 7 dimensions, and exactly equal in 3 . Comparing the $\frac{1}{8}$ wild males with the guinea-pig, it was found that they were slightly larger in 13 of the 16 averages, whereas the females were slightly smaller in 15 of the 16. Here again, the differences must be cautiously interpreted, for they were small in comparison with the probable errors and especially in comparison with four times the probable error. The differences between the $\frac{1}{8}$ wild and the guinea-pig were extremely small, and not apparent to the naked eye, as the figures of average dimensions show. Irrespective of whether or not we consider the $\frac{1}{4}$ wild smaller than the guinea-pig, it is quite certain that two back-crosses made the $\frac{1}{8}$ wild the equal of the guinea-pig in size. 
Summing up the data bearing on average skeletal dimensions in $C$. rufescens, C. porcellus, and three generations of hybrids, we may say that:

(1) C. rufescens is smaller than C. porcellus.

(2) The $\frac{1}{2}$ wild hybrids were larger and more vigorous than either parent species.

(3) The $\frac{1}{4}$ wild were smaller than the $\frac{1}{2}$ wild and possibly showed a general tendency to be smaller than the guinea-pig, particularly in the male sex.

(4) The $\frac{1}{8}$ wild and the guinea-pig were of the same size and practically indistinguishable.

\section{Coefficients of Variability of Dimensions.}

C. rufescens is specifically smaller than C. porcellus. We do not know whether the smaller species lacks factors for size, or whether it has factors inhibiting growth, or whether there are any "factors" involved at all. If we suppose that the difference in size is due to one or many completely dominant factors, then the $F_{1}$ should be like the dominant parent; and crossing the $F_{1}$ and $F_{2}$ generations back to this parent should give only the dominant form. But if we suppose the difference to be due to multiple, incompletely dominant factors, then the $F_{1}$ generation should be a blend, and the $F_{2}$ should show an increased variability, as was shown on pages 50-51. It has been pointed out by East (1910) that "as dominance becomes less and less complete, the Mendelian classes vary more and more from the formula $(3+1)^{n}$ and approach the normal curve, with a regular gradation of individuals on each side of the mode." In order to ascertain whether the hybrids were more variable than the parents, the coefficients of variability were calculated (see tables 67 and 68).

The variability of $C$. rufescens is unknown. The number of adult skeletons available in our own experiments was far too small to use as data. If we analogize with the tame C. porcellus, it is probable that the wild is not very variable.

The coefficients of variability of the guinea-pig were extremely small. The highest coefficient of any dimension in either sex was only 3.73 per cent \pm 0.20 (measurement 9 , table 67 ). Only 6 of the 32 coefficients were 3 per cent or more. Furthermore, they were very uniform, for the lowest was exactly 2.00 per cent \pm 0.12 ; and they range, therefore, from 2 per cent to 3.73 per cent. Compared with the parent stock used in experiments on maize (Shull 1910, East and Hayes 1911), or with the stock used in experiments on gourds and beans (Emerson 1910), these coefficients in the guinea-pig are very small. In the case of maize, the coefficients of variability of the parents were sometimes as large as 14 per cent. Emerson gave a coefficient of variability as 26.9 per cent for the shape of one parent (scallop) in summer squashes. This in no way reflects on the results and interpretations of these 
investigators; but the comparison is interesting and shows how uniform the skeletal dimensions of adult guinea-pigs really are. It is probable that the wild cavy species is likewise very uniform.

The coefficients of variability of the one-half wild hybrids were calculated from such small numbers ( 5 males and 8 females) that their value is doubtful. Such coefficients are most valuable and accurate when the number of variates is large. When the total number of variates is small, a few wide deviates greatly increase the standard deviations, and therefore increase the coefficients of variability also. According to the theoretical scheme involving multiple, independent size factors, incompletely dominant, the $\mathrm{F}_{1}$ generation should be a blend and no more variable than the parents, if the parents were practically pure. As a matter of fact, the $\frac{1}{2}$ wild were larger than either parent. We say that such phenomena accompany the heterozygous condition, but we can not adequately explain it. Taking the coefficients as they stand, the variability of the $\frac{1}{2}$ wild females was slightly greater than that of the guinea-pig parent, but the male hybrids were on the whole no more variable than their parent. In view of the fact that the chances of error are great, no conclusions can be drawn.

The one-quarter wild hybrids, or $\mathrm{F}_{2}$ generation, showed no great increase in variability, as one would expect on the hypothesis of many interchangeable factors without dominance. The males were no more variable than the guinea-pig, and the females were only slightly so. Here, again, the numbers were small (16 males and 20 females) and the results are subject to a serious objection.

The one-eighth wild hybrids, or $\mathrm{F}_{3}$ generation, were on the whole only slightly more variable in both sexes than the guinea-pig.

It can be readily seen that all the coefficients of variability are small and form no series consistent with the hypothesis advanced, according to which the $F_{1}$ generation should be no more variable than the parents, but the $\mathrm{F}_{2}$ generation should show an increased variability, while the $\mathrm{F}_{3}$ should be less variable than the $\mathrm{F}_{2}$ generation. The whole 128 coefficients in tables 67 and 68 are very small and close together. Moreover, if one considers the probable errors, the chances are small that the differences in variability are not due to random sampling. Practically every coefficient in any particular dimension would overlap every other one in that dimension if the probable error is multiplied by four. Therefore, from the standpoint of pure random sampling, the chances are large that a repetition of these experiments, under similar conditions and involving the same numbers, might easily give results with no significant differences between the coefficients of variability. It must be stated that probable errors for the $\frac{1}{4}$ and $\frac{1}{2}$ wild are very unreliable, since the numbers are so small.

Examining the data as they stand, to ascertain which dimensions . show a series of coefficients most variable in the $\frac{1}{4}$ wild and grow less 
variable as they approach the guinea-pig, we find such to be the case for the males in measurements 3,6 , and 10 , and for the females in measurements 3,5 , and 8 to 16 . (The $\frac{1}{2}$ wild are not considered on account of the small numbers.) Now, if we had by chance chosen only measurements 3 and 10 as the basis for our comparisons, then we would have been led to the conclusion that there was consistent evidence of segregation and a recombination of size factors in both sexes. But had we chosen other measurements we might have arrived at different conclusions. The question naturally arises, are the series of coefficients in any one dimension more significant than those in any other? Are we justified in selecting particular series which conform to the results presented by other investigators, and thus indicate a recombination of factors? As far as we can tell, we are not; for at present we know of no reason why special emphasis should be attached to the results obtained in certain measurements in preference to others.

There is another method of approach by which it is possible to avoid attaching questionable weight to a few dimensions. We may average all the coefficients of variability in each of the different classes to see, for example, if the $\frac{1}{4}$ wild were on the whole more variable than the guinea-pig. Table 69 gives the averages of the different coefficients of variability in the guinea-pig and hybrids, the purpose being to ascertain what the general tendencies of any class might be and to see whether on the whole the hybrids showed a general tendency to greater average variability than the parent guinea-pig. We also wished to see if, on the whole, the $\frac{1}{4}$ wild were more variable than the $\frac{1}{8}$ wild and the guinea-pig. However, the male $\frac{1}{4}$ wild averaged no more variable than the guinea-pig; but the female $\frac{1}{4}$ wild were more variable. All the different classes of males were of equal average variability except the $\frac{1}{8}$ wild. All the female classes were statistically of equal average variability except the female guinea-pigs. The males do not show a series indicating that the $\frac{1}{4}$ wild average most variable and that this variability decreases as we approach the guinea-pig; but the females do. In other words, there is little, if indeed any, evidence of segregation and recombination of factors for size in these crosses.

It is interesting to note that the $F_{3}$ hybrids ( $\frac{1}{8}$ wild) of both sexes averaged more variable than the guinea-pig. These expressions of average variability were based upon 16 different coefficients of variability. Back of each coefficient of variability there were from 60 to 78 variates. If one interprets the data from a purely statistical point of view, then the $\frac{1}{8}$ wild hybrids were inherently more variable than the parent guinea-pig and the chances are enormous that this difference is not due to random sampling. However, in interpreting biological data, other considerations are of importance. It is shown that all the coefficients of variability in the $\frac{1}{8}$ wild and the guinea-pig are extremely small. The averages of the guinea-pigs and $\frac{1}{8}$ wild, in tables 63 and 64 , 
were shown to be practically the same; and, hence, a difference of less than $1 \mathrm{~mm}$. in the standard deviation of any measurement would completely obliterate the differences in the coefficients of variability. Although I have undertaken no experiments to ascertain the effect of environment on skeletal dimensions, experience with many hundreds of guinea-pigs and hybrids leads me to believe it would be decidedly strange if environment could not effect a difference of less than $1 \mathrm{~mm}$. in the standard deviation of the guinea-pigs and hybrids.

Summarizing the facts concerning variability in the guinea-pigs and hybrids, we may say that-

(1) The variability of all the classes of hybrids and the guinea-pig was very small.

(2) There were no great differences in variability in the back crosses of hybrids to guinea-pigs which would indicate segregation and recombination of factors for size. This is true for the individual measurements and for the general average variability of each class.

(3) The results in no way controvert the possibility that size may be due to factors which are inherited in Mendelian fashion; but segregation was not apparent in these classes of matings in this species cross. The dominance of the guinea-pig may well be very nearly complete. Since the hybrids were mated back to the guinea-pig each time, it is simply a case of dominance with little or no evidence of segregation. According to this explanation, the vigorous growth of the first, or $\frac{1}{2}$ wild, hybrids was due to their heterozygosity, but without the effect of heterozygosis they would have been a little smaller than the $\frac{1}{4}$ wild. Mating the $\frac{1}{2}$ wild to the guinea-pig raised the mean of the $\frac{1}{4}$ wild nearly to that of the guinea-pig and a second back-cross raised the mean of $\frac{1}{8}$ wild right up to the guinea-pig. If the guinea-pig is dominant, or almost so, one would expect little or no evidence of segregation.

(4) It would be interesting to know whether the small $C$. rufescens was derived from a larger species such as $C$. aperea, $C$. cutleri, or $C$. porcellus by the loss of size factors, or whether the larger species arose by progressive variations from this small wild species.

\section{THE SKULL SUTURES.}

Among other characters which differentiate the wild $C$. rufescens from the guinea-pig, the nasal-frontal suture and frontal-parietal suture appear to be prominent. In the wild, the suture between the nasal and premaxillary bones and the frontal bones forms an $M$. The caudal margin of the nasal bones forms a $V$, and with the premaxillaries the whole suture is more or less M-shaped. In the tame, this suture is approximately truncate. The suture between the frontal and parietal bones in the wild is practically a straight line; but in the tame this same suture dips distinctly backward (see figs. 10, 11, 15, 16 , and 31). 
No satisfactory measure of the sutures could be found and, therefore, camera-lucida tracings were made of the nasal-frontal suture in all available skulls. The original data are presented directly in figures 42 to 47 . Drawings were made as shown in the table herewith.

Fifty-three camera lucidadrawings of this suture in the guinea-pig are given in figure 42. Several hundred skulls were examined, but no cases were found which could be confused with the wild (fig. 43). There is a

\begin{tabular}{rr}
\hline C. rufescens........ & 6 \\
C. porcellus....... & 53 \\
$\frac{2}{2}$ wild hybrids.... & 13 \\
$\frac{1}{4}$ wild hybrids.... & 44 \\
$\frac{1}{8}$ wild hybrids.... & 133 \\
$\frac{1}{16}$ wild hybrids.... & 189 \\
Total......... & 438 \\
\hline
\end{tabular}
range of variability in the tame; but in general the suture may be described as forming nearly a transverse line. Only 6 C. rufescens sutures are shown. We do not know whether the wild is very variable or not. Nor do we know that the wild males used in the crosses were pure for such a character. When the wild males were mated with tame females, the $\frac{1}{2}$ wild (fig. 44) showed the effect of the wild parent. None of the $13 \frac{1}{2}$ wild were truncate, but all were Mshaped.

The $\frac{1}{2}$ wild females were mated to guinea-pig males. Their $\frac{1}{4}$ wild offspring were very variable. Forty-four of these showed a range of forms from those like the $\frac{1}{2}$ wild to forms just like the tame (see fig. 45). It may mean that there was a rearrangement of factors, and the tame form segregated out in this $\mathrm{F}_{2}$ generation, as one might expect on the basis of several incompletely dominant factors.

The $\frac{1}{4}$ wild females were mated with guinea-pigs to produce the $\frac{1}{8}$ wild, and these in turn were mated to guinea-pigs to produce the $\frac{1}{16}$ wild. The $\frac{1}{8}$ wild (fig. 46) and $\frac{1}{16}$ wild (fig. 47) presented a wide range of forms. This was to be expected, for the hybrid females used as dams were of many very different types. No series of guinea-pigs, to my knowledge, ever showed such a range as these hybrids.

If the wild form is regarded as dominant, then the perfectly truncate forms which segregated out in the $\mathrm{F}_{2}$ (or $\frac{1}{4}$ wild) might be expected to breed true when mated back to the recessive guinea-pig. This was not found to be the case; for some of these female hybrids with perfectly truncate sutures had offspring showing M-shaped sutures. In other words, those $\mathrm{F}_{2}$ individuals which appeared to be recessive often gave $\mathrm{M}$-shaped sutures in the $\mathrm{F}_{3}$ generation. It is difficult to say whether or not this was due to the interaction of complementary factors. The number of offspring from each $\mathrm{F}_{2}$ female was necessarily small. Some bred true to the recessive truncate form, others did not.

The frontal-parietal suture of the wild was also apparently dominant in the $F_{1}$. The $F_{2}$ generation was variable, giving some segregates like the tame (see figs. 12, 13, 14, 17, 18, 19, 32, and 33). 


\section{MISCELLANEOUS MORPHOLOGICAL CHARACTERS.}

\section{THE INTERPARIETAL BONE.}

An interparietal bone occurs in young guinea-pigs, but after a few weeks it generally becomes fused with the parietals and can not be detected. We do not know whether it ever occurs in the adult wild. Table 70 shows its occurrence in the wild, tame, and hybrid guinea-pigs which were available for study. Figures 13, 18, 19, and 33 show its form, usually a very distinct triangular bone. Its occurrence in guineapigs is infrequent. It occurred in 9 out of 141 guinea-pigs, or 6.4 per cent. None of these guinea-pigs were used in matings with the wild or hybrids. Among the $\frac{1}{2}$ wild it was found in two cases, or 15.4 per cent. These two cases were a brother and sister, but none of the subsequent hybrids showing an interparietal bone were descendants of these two.

The interparietal was present in 15 out of $46 \frac{1}{4}$ wild hybrids, or 32.6 per cent. Eight of the $9 \frac{1}{4}$ wild females showing it were mated with the guinea-pig, and 5 of them had some offspring which also showed it. But other $\frac{1}{4}$ wild females had offspring which showed the same anomaly. In other words, some of the $23 \frac{1}{8}$ wild hybrids showing an interparietal bone were descended from females which had it, while others were descended from females showing absolutely no trace of it. The interparietal seemed to be most frequent (32.6 per cent) in the $\frac{1}{4}$ wild, and when these were mated to guinea-pigs the $\frac{1}{8}$ wild showed it in 18.4 per cent. One would expect it to decrease in frequency, for continually mating back to the guinea-pig should eventually establish the zygotic constitution of guinea-pigs in most dilute hybrids, and thus reduce the frequency of an interparietal bone to that of a race of guinea-pigs.

\section{THE SHAPE OF THE SKULLS.}

The skull of the wild $C$. rufescens is specifically much more pointed than that of $C$. porcellus (see figs. 10, 11, 15, 16, and 31). In crossing these two species, the $F_{1}$, or $\frac{1}{2}$ wild, was an apparent blend (see figs. 12,17 , and 32). Crossing the $F_{1}$ generation back to the guinea-pig gave some forms just like the guinea-pig, although most of them showed traces of the wild influence (see figs. 13, 18, and 33). The next backcross, giving the $\frac{1}{8}$ wild or $\mathrm{F}_{3}$ generation, were in general similar to the guinea-pig, but possibly showed a little wider range.

To ascertain the magnitude of pointedness or triangularity of a skull is difficult. If one takes the ratio of the greatest width of a skull to the width at the laterocaudal margin of the infraorbital foramen, one obtains an idea of the triangularity; but the quotients thus obtained can not be regarded as more than approximations. Measurements 9 and 11 were the widths of the skulls at these two levels. Dividing measurement 9 by measurement 11 gives an index of the triangularity; 
for, the more pointed a skull is, the greater will be the quotient, provided the distance between these two transverse measurements remains the same. The sagittal length of the skull between measurements 9 and 11 is in reality the altitude of a trapezoid, of which these widths are the bases. There are two ways of dealing with the pointedness of these skulls. One can take the ratio of the averages of measurements 9 and 11, given in tables 63 and 64, or, one can take the average of the ratios of measurement 9 to 11 in the individual skulls. Ratios of averages and the average of ratios are not necessarily the same, to be sure. The first case would mean the pointedness of an average or ideal skull in a given class, and the second case would mean the average pointedness of an array of many skulls in this class. Both sets of quotients were calculated and are given in table 71. They are practically the same, and this is probably due to the high degree of correlation between measurements 9 and 11 in any given class.

The indications are that:

(1) The wild was more pointed than the tame.

(2) The $\frac{1}{2}$ wild were an apparent blend.

(3) The $\frac{1}{4}$ wild, according to the table, were the same as the $\frac{1}{2}$ wild; but as a matter of fact they were less pointed. The $\frac{1}{2}$ wild skulls were very large, and since the distance between the two widths (the altitude of a trapezoid) was longer, the same ratio must mean that the $\frac{1}{2}$ wild were more pointed than the $\frac{1}{4}$ wild.

(4) The $\frac{1}{8}$ wild were approaching the guinea-pig-skull shape.

The coefficients of variability for the ratios of measurement 9 to 11 were calculated; but like the coefficients of variability for the linear dimensions, they were small and showed no significant differences. However, there is no doubt but that individuals were obtained in the $F_{2}$ and $F_{3}$ generations which were identical with guinea-pigs. Possibly we are justified in regarding these as segregates, due to the recombinations of factors.

\section{EFFECT OF STERILITY IN THE MALES.}

Throughout the discussion the sterility of the males has been neglected. In the case of non-functioning testicles it has been shown that ossification is delayed, particularly in the long bones. Recently Geddes (1910) has shown this to be the case in pathological conditions as well as in castration. The measurements of all hybrid males in table 63 were taken from fully sterile animals (except two males). By sterile we mean that they lacked motile spermatozoa and were incapable of fertilizing an egg. In many cases they showed no spermatozoa at all in the epididymis. The averages and variability of these sterile $\frac{1}{8}$ wild males are so close to the guinea-pig that it may be safely concluded there was no effect from such sterility. The number (60) of instances is large enough to make the average significant. That 
the testicles were entirely non-functional can not be maintained, for the cells of Sertoli, the interstitial cells, and spermatocytes may have been present. These may exercise some normal functions. A cytological study will be undertaken later. The $\frac{1}{8}$ wild females were fertile and also equal to the guinea-pig in size. Therefore, the $\frac{1}{8}$ wild of both sexes average the same as the guinea-pig, and the peculiar sterility of the males has no effect, similar to that reported by Geddes. The sterile $\frac{1}{4}$ wild males are actually smaller than the guinea-pig.

The difference between these sterile males and those in Geddes's experiments is that, in the former, the testicles were present and may have functioned in secreting hormones; whereas in the latter case they were really entirely non-functional. That the testes of sterile male hybrids were partially functional we are quite certain, for the secondary sexual characters were all present. The prostate glands and seminal vesicles were perfectly well developed. Sixteen hybrids were castrated at the age of 3 weeks, for the sake of comparison. Their seminal vesicles were greatly atrophied and they showed no sexual instinct throughout life. All these facts lead us to believe that the sterility of the male hybrids is not comparable at all to that sterility due to pathological conditions, kryptorchism, and castration. It is not surprising then, that the long bones of the sterile $\frac{1}{8}$ wild male hybrids and the male guinea-pigs were of equal length.

\section{ANOMALIES OCCURRING IN THE HYBRIDS.}

In addition to the frequent occurrence of the interparietal bone, peculiar to the hybrids, there were a number of other anomalies which should be mentioned.

(1) The wild C. rufescens and the guinea-pig have 4 toes on the front feet and 3 on the hind feet. By selection, Castle (1906) was able to produce a race of guinea-pigs having 4 toes on the hind feet. There occurred among the $\frac{1}{8}$ wild a male ( $\sigma^{7} 202$ ) with 5 well-developed functional toes on the left front foot and left hind foot. Like most males of this blood, he was sterile. The anomaly was never repeated. This may have been a reversion to the ancestral pentadactylous condition, brought about by recombining factors. It is interesting to note that the extra toes occurred on the left side, for Castle found that the extra toe in his polydactylous race was more frequent on the left side also.

(2) There occurred some monstrosities in the hybrids which I have never seen in guinea-pigs, although many hundreds have been carefully studied. In one of the hybrids the first cervical vertebra, the atlas, was completely fused with the skull. In another hybrid both scapulæ were bent so as to form a sharp angle, whereas normally they should be flat. In two female hybrids ( $\% 263$ and $q 393$ ) the clitoris 
was greatly enlarged and possessed the two lateral horns at the distal end which characterize the penis. Their sexual propensities are discussed in Part III. A female $\frac{1}{16}$ wild hybrid had large caudal vertebræ which, although normal in number and shape, formed a small tail about half an inch in length.

In the absence of more data relating to these and other anomalies, one can only speculate as to their cause and significance.

\section{GENERAL CONCLUSIONS AS TO GROWTH AND MORPHOLOGICAL CHARACTERS.}

(1) The wild C. rufescens used in these crosses were about half as large as the guinea-pig, C. porcellus. They were not only less in weight, but their bones were also shorter and more slender. The $\frac{1}{2}$ wild hybrids were usually heavier at all ages, had larger skeletal dimensions, and gave every indication of being more vigorous than either parent species. The $\frac{1}{4}$ wild hybrids lacked this vigor, for they were smaller than the $\frac{1}{2}$ wild hybrids in every way. They were very nearly the equal of the guinea-pig in average size and skeletal dimensions. Possibly the males were a little smaller than the guinea-pig. The $\frac{1}{8}$ wild hybrids averaged about the same as the guinea-pig in weight and skeletal dimensions. Two back-crosses were sufficient to render the $\mathrm{F}_{3}$ hybrids and guineapigs practically indistinguishable in size and skeletal dimensions.

(2) The number of adult wild available was too small to give a satisfactory index of their variability. The same was true of the $\frac{1}{2}$ wild hybrids. The guinea-pigs were remarkably uniform. The variability of all hybrids in both sexes was very low and gave no clear indication of segregation.

(3) The M-shaped nasal-frontal suture of the wild appeared to be dominant. Crossing back to the tame species gave a wide range of variability in the $F_{2}, F_{3}$, and $F_{4}$ generations. The truncate nasalfrontal suture of the tame species was recovered in the $F_{2}$ generation or $\frac{1}{4}$ wild, but did not breed true.

(4) The differences in skull-shape between the wild and tame were blended in the $F_{1}$ generation. In later generations all traces of the pointed, wild skull-shape were gradually lost. The deep, narrow indentation on the outer surface of the last upper molar, almost separating the small third lobe from the body of the tooth, was reduced in the $F_{1}$ generation; and all traces of it were lost in later generations. The taxonomists lay great stress on this character.

(5) There was no apparent effect of sterility on size in the male hybrids.

(6) The unusual frequency of an interparietal bone, the occurrence of a 5-toed individual, and other anomalies were observed in the hybrids but not in the guinea-pig. 



\section{PART III. THE FERTILITY OF THE PARENT SPECIES AND HYBRIDS.}

\section{INTRODUCTORY DISCUSSION.}

When the wild Brazilian male cavy, Cavia rufescens, was crossed with the tame domestic female guinea-pig, Cavia porcellus, the hybrids were fertile females and sterile males. At least three problems were immediately self-apparent: for how many generations would the hybrid females have to be crossed back to the parent males before producing fertile hybrid males; what proportion of sterile males would the more dilute wild hybrid females produce; and when fertile hybrid males were produced, would their offspring be fertile in both sexes if these males were mated with their hybrid sisters or with guinea-pig females.

Sterility is a common phenomenon in the hybrids obtained by crossing individuals belonging to distantly related groups or types, both in animals and in plants. In fact, there is a tacit understanding among biologists that members of the same species produce fertile offspring; but a successful cross between members of different species or genera may result in sterility of the hybrids, in one or both sexes. In case both sexes in a species cross are sterile, a continuation of the genetic investigation becomes impossible. If one sex alone is sterile, then the fertile sex can be crossed back to either parent species, and it becomes possible to study the inheritance of various other characters as well as their fertility and sterility. In the experiments recorded in this paper, wild $C$. rufescens males were mated with the tame guinea-pig females and produced fertile female and sterile male hybrids. The fertile hybrid females were crossed back to the males of both parent species. The back-cross to the wild $C$. rufescens males succeeded in so few cases (four offspring were produced) that this class of matings had to be abandoned. The back-cross to the guinea-pig males was entirely successful. The $\frac{1}{4}$ wild females alone were fertile, and a second back-cross to the guinea-pig produced the $\frac{1}{8}$ wild. In this manner there were produced ten generations of hybrids, by repeatedly crossing female hybrids of one generation back to guinea-pigs to obtain the next more dilute wild-blooded generation. The results of these crosses have been studied with regard to coat, color, growth, size, and morphological characters and recorded in Parts I and II of this paper. The same animals were used in studies on fertility and sterility.

Bateson (1913), in his review of "Mendelian segregation and species," is inclined to the view "that successful investigation of the nature even of sterility consequent on crossing, the most obscure of all genetic phenomena, may become one of the possibilities of Mendelian research." The material presented in this part of the series of studies in a mam- 
malian species cross deals mainly with sterility in the male sex, consequent on crossing.

That such complicated physiological phenomena as fertility and sterility in all kinds of crosses and under all conditions can be discussed or treated solely as problems in heredity is out of the question. Probably no one would insist that fertility or degrees of fertility always depend upon "factors" or "germinal determiners." However, it does not follow that in certain crosses factors may not be transmitted in Mendelian fashion which influence the fertility of the hybrids. On $a$ priori grounds we have no reason to suppose that all cases of varying fertility and sterility are due to environmental conditions; for, although environment undoubtedly influences fertility, there are unquestionable instances in which the results may be ascribed to other causes.

There seems to be little doubt that environmental conditions may affect the fertility of one or both sexes, and this should be carefully considered when we are dealing with the inheritance of the same. Marshal (1910) states: "it is well known that wild animals, when removed from their natural conditions and brought into captivity, often become partly or completely sterile." He cites cases from different groups of mammals and birds. Darwin (1876) also drew attention to this fact. Both of these investigators recognized that animals differ widely in this respect. The Indian elephant, chetahs, some carnivores, some rodents, monkeys, hawks, finches, parrots, and many other cases show sterility; but one can not generalize hastily and infer that all changes from a wild state to captivity result in a lowered fertility, for it is also known that certain gallinaceous birds, ostriches, pigeons, ducks, geese, and gulls, and some mammals like the skunk, ferret, mink, and Cavia aperea will breed readily in captivity. It is often asserted that wild animals in captivity are sterile because of change in diet, temperature, surroundings, lack of exercise, and the like; but none of these factors necessarily causes sterility, for one can always cite contradictory evidence.

It is no easy task to differentiate between the effect of environmental factors and hereditary factors, particularly when the influence of the different factors is small and their number is large. In any comparison between the fertility of the wild $C$. rufescens, the domestic guinea-pig, and the various hybrids, a number of environmental factors should be given careful consideration, since it may be supposed that the wild species underwent a great change when transferred from its native habitat in Brazil to the laboratory of the Bussey Institution. All of the causes which are cited as disturbing fertility appeared to be of little or no consequence in these crosses, for it will be shown that the wild were apparently quite fertile inter se; and the wild males were surely fertile in crosses on tame females. The change from a wild habitat with the concomitant changes in diet, temperature, surround- 
ings, and the like did not prevent the wild females from breeding. The wild males, as previously stated, could only be mated to tame females with difficulty; and yet, when successful matings were secured, these tame females bore the usual average per litter characteristic of the guinea-pig. This shows that the wild males produced an abundance of spermatozoa and fertilized the usual number of eggs, exactly the same as a tame male would have done.

A study of the fecundity of the wild, tame, and hybrid females will show whether or not we are justified in concluding that environment has played little or no part. No attempt is being made to underrate the effect of environment upon fertility, for it is recognized that nutrition, age, change of surroundings, temperature, drugs, disease, and the like may exercise profound effects. However, since the wild breed in captivity and the wild males are fertile in crosses with guinea-pigs, captivity itself may be eliminated as a factor causing sterility in the less wild hybrid sons. The original wild male $\left(o^{\top} 1\right)$ lived and bred in captivity from 1903 to 1908 - a period of almost 5 years. The great difficulty with these wild in captivity was not that their wildness prevented fertility, but that their nervous, excitable disposition made them difficult to handle and led to injuries in one way or another. Nehring experienced little or no trouble with wild $C$. aperea in captivity and they remained fertile at the same time.

We do not know what the exact fertility of the wild $C$. rufescens may be in its native habitat, nor have we any basis upon which to compare its fertility in the wild state with its fertility in the laboratory pens. There are some observations by naturalists upon the fertility of $C$. aperea in the wild state, but they are meager and contradictory. Nehring found that this species was more prolific in captivity than it was reported to be in the wild state. The wild C.rufescens, which were bred in captivity, aborted their young in a few cases. Abortion is, of course, not infrequent in the domestic guinea-pig, but I am inclined to believe that these abortions were more frequent in the wild cavy. The abortions may possibly be supposed to indicate a degree of disturbance in the sexual functions and signify a tendency toward sterility. If this is true it is the only evidence of any lessened fertility in the wild due to captivity. The abortions ceased in the hybrid females, and there were no other signs of any sexual disturbances in the later, more dilute wild hybrids, other than the sterile males previously mentioned. The pure wild were very easily frightened, and when disturbed would run about frantically. It is not impossible that the abortions were caused by these violent paroxysms of fear and the subsequent effects on fœtal nutrition and other functions.

The fertility of the other parent species, the tame guinea-pig, is well known. Under the excellent conditions of housing, food, and care in our laboratory, a sterile guinea-pig is very uncommon. Of all males 
which came under my observation, there were only two which failed to breed. When the contents of the epididymis were examined it was found that they had an abundance of live, motile spermatozoa. Their impotence may have been due to sluggishness and a failure to copulate rather than to innate sterility. Female guinea-pigs in good condition are rarely sterile.

In view of the foregoing facts it would seem that the problem of sterility in the male hybrids in these crosses was fundamentally a problem of physiology and heredity, and not one of environment. The facts may be summarized as follows:

(1) The wild cavy species was fertile in both sexes in captivity.

(2) The tame domestic species was likewise fertile under the same conditions.

(3) The hybrids resulting from a cross between these two species were not like either parent, for they were sterile males and fertile females. Nevertheless these hybrids were very vigorous, as was shown in Part II.

(4) The peculiar sterility of the males persisted in later, more dilute wild generations in a manner which will be described subsequently. These later hybrids, however, could not be distinguished from the tame guinea-pig in shape, size, growth, mental traits, or any other characters, except their peculiar sterility. Therefore, since the wild were difficult to raise in captivity, but were fertile, and since their lesswild hybrid sons were easily raised in captivity but were sterile, it would appear that their sterility is not due to captivity or environment. If the facts have been correctly interpreted, some sort of consistent explanation should be found, based on heredity. The cross resulted in a definite disturbance in fertility such as did not obtain in either parent species when kept under the same conditions.

Many species crosses have been made in both plants and animals. In most cases the crosses were made by those who were merely interested in the sheer possibility of a cross, but not for the purpose of an extended genetic study. Much of the literature deals with the subject of sterility from a taxonomic point of view, for the fertility or sterility of the hybrids is considered a criterion of the close or distant relationship between the parents. From time to time compilers have given lists of species crosses with brief mention of the partial or complete dominance of one parent and the fertility of the hybrids when known. As in most other genetic studies, the botanists have led the way, and the studies of the early plant hybridists include many accounts of species crosses, or at least what were regarded as "species" crosses. Very complete summaries of species crosses in plants were made by Gärtner (1849) and Focke (1881). Numerous crosses have been made since, but in all the crosses between varieties or between species but few of them deal with the inheritance of fertility and sterility. 
Bateson and Punnett (Bateson 1913) have reported a case of simple Mendelian inheritance of sterility in sweet peas, in which normal anthers were dominant to sterile anthers. The case is complicated by coupling with a color factor.

Biffen (1905) crossed species of barley having well-defined grades of fertility. His results showed that the hooded barleys, Hordeum trifurcatum and $H$. hexasticofurcatum, which are more fertile than the normalawned barleys, were dominant to four different species of the latter kind. Segregation took place and it was inferred that only one allelomorphic pair of characters was involved. In other crosses between well-defined types of barley he found various kinds of sterility dominant over the normal perfectly developed floret. "In these cases the various degrees of sterility, ranging from complete suppression of the reproductive organs in the lateral florets to reduction in size only, are clearly dominant over the perfectly developed floret." Here, again, the classes obtained in the $\mathrm{F}_{2}$ generation gave evidence of a simple segregation.

Brainerd (1907), in his résumé of the interesting behavior of certain hybrids between violet species, reports that pronounced degrees of sterility occurred in some of the crosses. When the hybrids were mated inter se he recovered plants of normal fertility in the $\mathrm{F}_{2}$ generation. In discussing the phenomenon of this segregation of normally fertile strains from an almost sterile hybrid $F_{1}$ generation, he says:

"With this diminution or entire loss of hybridity, we should expect a partial or total recovery from the impairment of fertility produced in the first cross. At any rate, it is an observed fact that many violet seedlings whose hybrid parents produced seed from only about one-tenth of their ovules, are themselves normally fertile."

We are still at a loss to know whether the fertility returned because there were recombinations of definite factors for fertility or because the simple recovery of parental types gave fertility like the parents. In the latter case the sterility of the $F_{1}$ hybrids might be thought to be due to disturbances arising from the admixture of widely diverse germinal elements, and a subsequent segregation of the parental types would mean a combination of factors and characters from one source, and with these the fertility of this parental type. But if fertility and sterility are due to independent factors, one should be able to combine the characters of either parent with fertility or sterility, or degrees of either.

DeVries (1909) found that Oenothera lata produced no fertile pollen, although it was normally pistillate. The anthers showed all conditions, from the absence of grains to normally developed pollen, but they were always sterile. He was able to fertilize $O$. lata with pollen from $O$. lamarckiana. The anther sterility was transmitted through the ovules of $O$. lata, but was coupled with other $O$. lata characters, for it segregated out associated with them. 
Bauer (1911) studied a cross between the self-fertile Antirrhinum majus with the self-sterile $A$. molle and obtained dominance of selffertility. The $\mathrm{F}_{2}$ generation split up into self-fertile and self-sterile forms, the majority being self-fertile, but the exact ratios were not determined. Since A. molle is never self-fertile, Bauer interpreted the phenomenon as physiological rather than mechanical. This case is a peculiar kind of sterility, inasmuch as the gametes are not sterile except in certain kinds of crosses. The inheritance of this peculiarity, nevertheless, follows Mendel's laws in its essentials. Bauer also reported a cross between $A$. siculum and $A$. majus which gave sterile ovules and fertile pollen. The pollen of these hybrids was capable of fertilizing A. majus, segregation taking place subsequently.

In plants, as in animals, the sterility following wide crosses is not of the same sort always, for sometimes both sexes are sterile or partly sterile, while in other cases one sex alone may be sterile or partly sterile.

The literature on species crosses in mammals is meager, particularly relatively to the inheritance of sterility. Compilations of species crosses in animals by Ackermann (1897, 1898), Rörig (1903), and Przibram (1910) give a fairly comprehensive conception of the amount of work done. One is reminded of Bauer's (1911) statement:

"Noch weniger als über Bastarde zwischen Pflanzen-species, sind wir über Artbastarde bei Tieren unterrichtet. Es sind zwar auch hier zahllose Artbastarde gelengentlich beobachtet oder auch künstlich erzeugt worden, aber eine auch nur einigermassen genügende $\mathrm{F}_{2}-$ Analyse est nie durchgeführt, ja überhaupt nie versucht worden."

Since so little is known of the inheritance of any characters in species crosses in animals, it is not surprising that nothing is known of the inheritance of sterility subsequent to such crosses. Sterility, to be sure, often accompanies wide crosses in animals. In the Lepidoptera the classical experiments of Standfuss (1895) have shown that such crosses may give partial or complete sterility in either sex, gynandromorphs, hermaphrodites, and even the complete suppression or elimination of one sex. Recently Goldschmidt (1912) has attempted, on a Mendelian basis, to explain gynandromorphism in the cross between Lymantria dispar with $L$. japonica, upon the assumption that the factors for the secondary sexual characters of the two parent species are of various grades of potency. For our purposes it is not necessary to enumerate all the species crosses resulting in sterility. These have been fully recapitulated, summarized, and described by other investigators (Poll 1910, 1911; Przibram 1910).

A few bovine crosses have yielded results somewhat similar to the cavy crosses in this paper. Kühn began a series of crosses, using the genera Bibos, Bison, and Bos. The original papers were not accessible, but a summary is given by Nathusius (1912). The yak, Bibos grunniens, has been crossed with the domestic cow, Bos taurus, and pro- 
duced sterile male but fertile female hybrids. The female hybrids were crossed back to males of both parent types; but the male hybrids remained sterile, although 19 were tested and included $\frac{1}{4}, \frac{3}{4}, \frac{1}{8}$, and $\frac{7}{8}$ domestic-blooded males.

The gayal, Bibos frontalis, has been crossed with the domestic cow and likewise produced fertile female but sterile male hybrids. At least $6 \frac{1}{2}$-gayal bulls were tested and found to be sterile, but 3 out of $9 \frac{1}{4}-$ gayal bulls were fertile.

The gaur, Bibos gaurus, considered a close relative to the gayal, was crossed with the domestic cow. A male hybrid was sterile to cows (although he covered 19), but, strangely enough, he was fertile with his own sisters.

The banteng, Bibos sondaicus, was crossed with the zebu, Bos indicus, and produced a sterile male. I have been told that the female hybrids are fertile, and regard the sources of information as reliable.

The bison, Bison americanus, has been reciprocally crossed with domestic cattle, but most successfully when a domestic bull is used. The hybrids, frequently called cattaloes, are sterile males and fertile females. The female hybrids have been crossed back to males of both parent species, thus producing $\frac{1}{4}$ and $\frac{3}{4}$ bison (Boyd 1908; Iwanoff 1911). The $\frac{1}{4}$ bison females are fertile, as may be expected. The $\frac{3}{4}$ bison females have not been fully tested, but are presumably also fertile. The $\frac{1}{4}$ bison males are not always fertile, for Boyd reports the appearance of but 1 out of 4 tested males. Iwanoff reports a fertile $\frac{3}{4}$ bison male and supposes, on purely theoretical grounds, that a mating of such a fertile male with a $\frac{1}{4}$ bison female would result in fertile $\frac{1}{2}$ bison of both sexes. Boyd has more recently reported other fertile hybrid males (Boyd 1914).

\section{THE FERTILITY OF THE MALE HYBRIDS.}

\section{MATERIALS AND METHODS.}

The first two generations of male hybrids (the $\frac{1}{2}$ and the $\frac{1}{4}$ wild) were few in number and could be tested thoroughly by mating them to guinea-pigs or to their fertile hybrid sisters. But since the number of hybrids to be tested increased so rapidly in the succeeding generations (see table 72) that facilities were lacking to mate all of them, it became necessary to resort to another method, if any knowledge of their fertility was to be acquired. In testing the fertility of hybrid males by breeding, it was necessary to keep them with four of five females for at least 4 months. Furthermore, it was found that much time and space were being wasted in trying to prove animals sterile or fertile by a breeding test, when a simple examination of the contents of the epididymis would show immediately whether it was useless to attempt to breed the hybrid. Therefore I decided to test each animal microscopically 
to ascertain whether or not a further breeding test should be applied. The value of the test is apparent, for out of 102 males tested microscopically 43 were found to have very few or no motile spermatozoa present and every one of them failed to breed after the most rigid breeding test. On the other hand, 44 males which proved to be fertile in breeding had an abundance of motile spermatozoa in every case.

The microscopic test was simple and expedient. A male tested in this manner was anæsthetized by etherization; the scrotum was thoroughly washed with 75 per cent alcohol, and dried; and the animal was stretched on his back. A small incision, or a cut made with scissors, about $\frac{3}{8}$-inch long, at the posterior end of the scrotum, exposed the edipidymis. Several of the tubules were then transected with a very small, sharp scalpel, and the liquid contents which collected were placed on a cover-glass. The cover-glass was transferred to a slide, on which a drop of physiological salt solution had been placed. The cover-glass, slide, and salt solution were, to be sure, always kept at bodily temperature. The slide was then examined under the microscope and a careful record of observations was made. There were 433 males of the different hybrid generations tested in this manner. In all cases a record was kept, showing which testicle has been used for operation. For the sake of convenience the left testicle was always used. Bilateral tests were made in enough cases to show that either testicle would give the same result; but such tests were made only after a thorough breeding test or with surplus animals, for transection of the epididymis on both testicles might make an animal sterile in breeding, although potentially fertile. The wound was covered with iodoform and healed completely in a week.

In order to exclude any possibility of varying tests on one and the same animal under different conditions, over 100 males were retested, both on the left side and on the right, in summer and in winter, and in good condition as well as in very poor condition. The second and third tests always gave the same results as the first, with the following exceptions: the cellular contents of the epididymis were always of the same character; but it must be stated that 3 males showed immotile sperm on the first test, but motile sperm on a second test some months later.* I am fully satisfied that the difference was due to my own early inexperience. The reverse never occurred, for when a second test showed immotile sperm after a first test had shown motile sperm, I could always locate the difficulty and immediately produce a repetition of the first results. Hence, I am inclined to believe that these 3 aberrant animals originally had motile sperm, and had simply failed to show it because the temperature of the slide was too low or because evaporation had

*The term, sperm, used to avoid frequent repetition of the cumbersome term, spermatozoa, will be clear from the context. 
concentrated the salt solution on the slide. The results showed that a careful microscopic test, at the age of 5 months or over, is a very reliable index of sterilty or fertility.

In order to test a male by breeding, it is essential that he should be healthy, and kept with vigorous adult females for a number of months. Even then a male may be potentially fertile, but fail to impregnate a female because of sluggishness or other external causes having no obvious relation to the mere presence or absence of motile sperm. The ideal test of fertility is the combination of a breeding and a microscopic test. There were, in all, 50 males tested by breeding alone, and 102 males tested in both ways. Whenever the breeding test was used a male was given every opportunity to demonstrate his fertility. The unreliability of a simple breeding test, however, was evident to me during the early part of the experiment, for a few males having an abundance of motile sperm failed to impregnate females, although continually with these for many months. Two such males were about to be given up as practically sterile after a breeding test of almost a year; but on deciding to continue the test I was greatly surprised and repaid by several litters from them. One of these two ( $\left.\sigma^{7} 375\right)$ did not impregnate a female until after 18 months of continued breeding. I suspect that some fertile hybrid males were not always as successful breeders as normal guinea-pigs, even though it was absolutely impossible to detect any difference in the abundance or character of their spermatozoa.

A total of 483 males was tested by one or both tests. The individuals ranged from the $F_{1}$ through the $F_{8}$ generation, most individuals (329) belonging to the $F_{3}, F_{4}$, and $F_{5}$ generations. The results are put in tabular form as far as possible and recorded in tables 73 to 77 .

Table 72 shows how many hybrid males in each generation were tested by either one or both methods.

THE RESULTS OF THE SIMPLE BREEDING TESTS ALONE.

About one-tenth of all the hybrid males were tested by a simple breeding test. They ranged from the $\frac{1}{2}$ wild to the $\frac{1}{16}$ wild, a total of 50 individuals (see table 73). The breeding test was thorough and there is no doubt that each of them, except one $\frac{1}{16}$ wild male ( $\left.0^{7} 305\right)$, was sterile for all practical breeding purposes. To be sure, some of them may have had immotile sperm or even some motile sperm, but they failed to impregnate any females as a normal guinea-pig would have done under similar circumstances. We have no knowledge of their germ cells. In the light of the other tests, these breeding tests became more significant.

The reason that so many hybrid males of the early generations were not tested microscopically was because the animals were scarce and valuable and it was feared that an operation upon the epididymis might 
destroy any even remote chance of successful breeding. Furthermore, at this period of investigation, facilities were available for mating the males, and the need of a more rapid and expedient test was not felt. The testes of some of these males were preserved for a later cytological study.

\section{THE RESULTS OF ALL MICROSCOPIC TESTS.}

Our knowledge of the fertility of about two-thirds of the hybrid males depends entirely on the examination of the contents of the epididymis (see table 74). Out of a total of 483 males, 331 were tested in this manner alone, and 102 males received both a breeding and microscopic test. The total number of microscopic tests was therefore 433 (see table 75). The results of the microscopic examination in those animals having both tests are given in table 76. For the sake of convenience, all microscopic tests will be discussed together, thus giving larger numbers from which to draw conclusions in table 75 . The hybrids are divided into four categories: with no evidence of sperm; with evidence of any sort of sperm; with any motile sperm; and with many motile sperm. A careful search made the first three classes easy to differentiate, but one must admit that there are no sharp class lines between the relative numbers of motile sperm. The classification "many motile spermatozoa" means that the examination showed an abundance of cells, all or practically all of which were motile sperm, being the same condition which prevails in the guinea-pig (see table 75).

(1) Hybrid males without spermatozoa.-Although the contents of the epididymis were taken from several tubules at different levels, and often from both testes, and at different times, some hybrids failed to reveal any spermatozoa or any evidence of such in the form of disintegrating flagella and the like. Such hybrid males, however, varied widely in the nature of their contents. The early hybrids without spermatozoa, such as the $\frac{1}{4}$ wild, usually showed a thin, clear, colorless liquid in the epididymis almost devoid of all cells, but hybrids of late dilute wild-blooded generations usually showed a thick, creamy liquid rich in cells and cell detritus. The cells present were apparently spermatogonia or spermatocytes, prematurely proliferated. The uniformity of the cells also differed, for some males had various kinds of cells, while in others all or most of the cells were apparently alike. In the later generations, the entire contents were often large, highly refractive cells, possibly spermatids, inasmuch as cells of this type were observed to have, occasionally, incipient tails. The tubules of the epididymis in the $\frac{1}{4}$ wild hybrids were thin and pale, but this condition became less and less frequent in later generations. The proportion of males without spermatozoa also gradually decreased. In general, we may say that the $\frac{1}{4}$ wild hybrids without spermatozoa showed a thin, clear liquid with a few small cells; but later generations 
showed increasing numbers of cells and more highly differentiated cells. The transition was gradual. It is probable that the cells were incompletely matured germ cells.

The $\frac{1}{2}$ wild male in tables 75 and 76 ( $\left.0^{7} 70\right)$ was examined from a histological preparation of the testis made by Dr. W. E. Castle.

(2) Hybrid males with spermatozoa.-All classes of hybrids, from the $\mathrm{F}_{2}$ generation on, contained some individuals showing spermatozoa. The difference between individuals was great, both in respect to quantity and character of sperm. Two $\frac{1}{4}$ wild males showed a few very imperfect, non-motile sperm mixed with a few of the usual cells. Twenty-two $\frac{1}{8}$ wild males likewise showed sperm, but in greater numbers and sometimes motile. The percentage showing sperm gradually increased, as would naturally follow, since the percentage without sperm gradually decreased. In the $F_{6}$ generation ( $\frac{1}{64}$ wild) about 96 per cent showed sperm. The $\mathrm{F}_{7}$ generation showed sperm in 87 per cent of the cases; but since the total number was only 15 , the results are subject to a valid objection. I am inclined to believe that larger numbers would have given a perfect series. When few sperm were present, only few other cells might be present also, as in the $\frac{1}{4}$ wild. In the later generations, if the sperm were infrequent, there usually was an abundance of other cells. Moreover, the sperm present varied in motility or might be misshapen or normal. If we simply consider the presence of any kind of sperm, table 75 shows that the percentage of males with sperm gradually increased as the wild blood became more dilute. The proportion with many sperm also gradually increased, while the proportion with few sperm decreased.

(3) Hybrid males with motile spermatozoa.-Hybrids showing sperm did not necessarily show motile sperm. Rarely a hybrid would have practically nothing but sperm, yet all of them immotile. Such animals would of course be sterile. In other cases hybrids showed only few sperm mixed with the usual cells, but all the sperm were motile. The variations between these two classes were continuous. The percentages showing any motile sperm whatever increased from 16.33 per cent in the $\frac{1}{8}$ wild to 86.67 per cent in the $\frac{1}{128}$ wild; and conversely, the proportion with no motile sperm gradually decreased in each generation after the $\frac{1}{4}$ wild.

(4) Hybrid males with many motile spermatozoa.-Males having but few motile sperm could not be bred successfully. This may have been due simply to the fact that there was less chance for a spermatozoon to reach an egg. I am inclined to believe, however, that mere abundance of motile sperm is not the only essential to fecundation, as will be shown later. It may well be that hybrids producing motile sperm sometimes fail to produce sperm qualitatively adequate. The greatest success in breeding was obtained with males showing an abundance of motile sperm. By abundance or "many motile sperm," as used in 
the tables, I mean, as stated above, that the epididymis was full of motile sperm and showed very few or no other cells. Males showing many motile sperm first occurred in the $F_{3}$ or $\frac{1}{8}$ wild generation. Although no previous hybrid generations had shown motile sperm, nevertheless, in this generation, 7 individuals showed a condition similar to that of any mormal male guinea-pig. The percentages showing many motile sperm increased from 14 per cent in the $\mathrm{F}_{3}$ to 73 per cent in the $\mathrm{F}_{7}$ generation.

THE RESULTS OF A COMBINED MICROSCOPIC AND BREEDING TEST.

The results of the microscopic tests have been discussed. About one-fourth of the animals tested in that way were also tested by breeding. Of the 433 males tested microscopically, 102 also had a breeding test (see table 76). The order of the test was not always the same, for about two-fifths of these males were bred first and then subjected to a microscopic test; but since the contents of the epididymis were the same under varying conditions, it should have had no effect on the results. For convenience, we may divide the animals into classes somewhat similar to those used in discussing the miscrocopic tests.

(1) Hybrid males without spermatozoa.-Twenty-three males of this type had been mated to females before a microscopic test was made. As was to be expected, none of them were fertile in breeding.

(2) Hybrid males with immotile spermatozoa.-Eleven hybrids with immotile sperm proved sterile in breeding. The number of sperm varied from a few in some cases to many or practically all sperm in others, but since all were immotile, they were, to be sure, completely sterile in breeding.

(3) Hybrid males with a few motile spermatozoa. - It is very difficult to classify males with motile sperm, since all grades existed, ranging from individuals with very few motile sperm to individuals with thousands of them. In all microscopic tests animals were recorded with reference to the number of sperm present and proportion of these that were motile. The relative number of sperm was described as "few," "half," "over half," and "all;" and the standard for "all" was the normal guinea-pig male or a completely fertile hybrid male. For example, a male recorded as "half" had sperm and the usual cells in about equal numbers, or he might have none of the usual cells but a deficiency of sperm. The motility was described in the records as " 1 ," " 2 ," " 3 ," and " 4. ." These signs had the following significance: " 1 " meant a few of the sperm present were motile; " 2 " meant half of the sperm present were motile; " 3 " meant over half of the sperm present were motile; " 4 " meant that all of the sperm present were motile. Obviously, this divided continuous variates into 16 crude classes. A male recorded as "half 4" had about half the usual number of sperm, 
but all were motile. A priori, one might expect "over half, 4," "all, 3 ," and "all, 4" individuals to be fertile in breeding. In the tables, all males recorded with "many motile sperm" were of the grade "all, 4." Any manifest departure from this condition is recorded in tables as having "few motile sperm." This will make clear that our records were more discriminating than our tables.

Using the term "few motile sperm" in the tables to mean any condition of number or motility plainly below that of a normal guineapig, we may say that 9 individuals out of a total of 10 were sterile in breeding. The exception was a $\frac{1}{8}$ wild male ( $\left.0^{7} 469\right)$ recorded as "over half, 2." This male had "over half" the usual number of sperm; but only half of these were motile. He was bred continuously for 9 months and sired one male. Possibly all males with any motile sperm whatever might have fertilized eggs had we increased their chances by using large numbers of females and long periods of mating.

(4) Hybrid males with many motile spermatozoa.-As previously stated, hybrids classified this way in the tables were as nearly like a normal guinea-pig as one could judge by examination of the contents of the epididymis. I expected they would prove to be just as fertile in breeding; but this was not the case, for some of them sired no young after a thorough breeding test. There were 7 males of this class among the $\frac{1}{8}$ wild; and all but one were successful sires. This exceptional male (o 721), large and vigorous, produced no young, although continually with fertile females for many months. Among the $\frac{1}{16}$ wild there were 22 males with many motile sperm, but only 16 of these were successful sires. The reason why the remaining 6 individuals were impotent is not clear; their weights and growth curves gave every indication of vigor; 3 of the 6 males were bred for the minimum time reasonably required to show fertility, and it is barely possible that the cause lay there; but this still fails to account for the remaining 3 .

Likewise among the $\frac{1}{32}$ wild, 6 males out of 24 had many motile sperm but failed to breed. Here again no evident reason, such as lack of vigor or early death, could be assigned to at least one of these cases. Of the $\frac{1}{64}$ wild males, 2 sired young, while one failed to-in all probability because of poor condition. Summarizing the results, there were 58 males with many motile spermatozoa, and 44 of these were successful sires. The remaining 14 individuals were sterile in breeding; of these 14 it is just barely possible that because of external causes 9 may have been sterile in spite of their abundance of motile sperm; but there was surely no patent cause for the sterility of the remaining 5 males. In other words, of 49 males (58 minus 9 ) which gave every indication of being fertile by a microscopic test and had opportunity to prove themselves so in breeding, there were only 44 which actually impregnated females. To state it differently, 89.8 per cent of the male 
hybrids with an abundance of motile sperm were actually fertile, while 10.2 per cent were sterile in breeding, a phenomenon which would not happen with normal guinea-pigs.

From this I conclude that the number and motility of the sperm are not the only essentials for a real fertility, inasmuch as real fertility in the last analysis must mean the capacity to fertilize eggs and sire young. There are further reasons for concluding that the motile sperm of hybrid males may be physiologically different from those of a normal guinea-pig; for it often required much more time to obtain young from the hybrid males, and the litters were unexpectedly small. In 129 litters from hybrid males, there were 238 young-an average of 1.84 per litter. The normal guinea-pigs produce about 2.4 young per litter. Some hybrid males produced large, vigorous litters, and others produced but few young after long mating. It was of course impossible to tell what proportion of the motile sperm formed were qualitatively complete in all essentials to perfect fertility; but undoubtedly some male hybrids with many motile sperm lacked other indispensable qualities, partly or completely. In addition, it may be stated that sterility was not due to the absence of the secondary sex characters, since all sorts of males, sterile or fertile, copulated and appeared otherwise normal.

THE INHERITANCE OF STERILITY.

Two species, fertile under the same conditions, were crossed and gave rise to sterility in the male hybrids. Some condition subsequent to hybridization disturbed gametogenesis in the males, but did not affect the females. The disturbing elements were carried and transmitted by the females, however, for crossing these back to the male guinea-pig gave sterile males again. After continued back crosses to the guinea-pig, increasing signs of fertility appeared and eventually completely fertile males were produced. The cause of the disturbance had, to all appearances, segregated out. One can hardly refrain from the thought that these fertile males segregated out in a Mendelian sense, and that there were a number of physiological factors involved and transmitted alternatively, the different recombinations of which gave the various expressions of fertility and sterility. To be concrete, had the sterility of the $\frac{1}{2}$ wild males been due to one simple factor, or to a group of completely coupled factors, or to disturbances between one homologous pair of chromosomes at some stage of reduction, then we should have expected 50 per cent of the $\frac{1}{4}$ wild males to be fertile. If the heterozygous condition of an allelomorphic pair, Aa, caused sterility in the $\frac{1}{2}$ wild males, but did not affect their sisters, then mating these females back to the tame, aa, would give 50 per cent $\mathrm{Aa}+50$ 
per cent aa, or fertility in one-half of the $\mathrm{F}_{2}$ males. In Mendelian notation it would be:

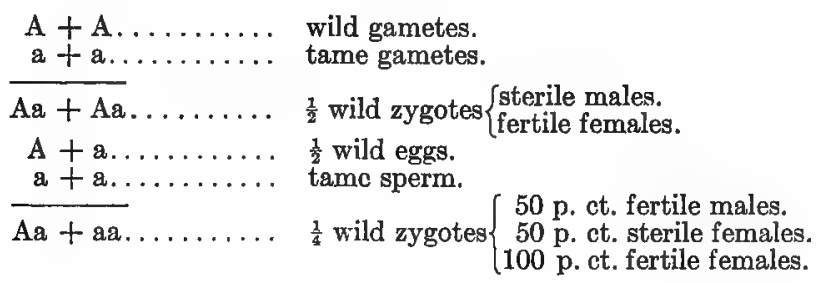

Furthermore, although all $\frac{1}{4}$ wild females would be fertile, half of them would transmit sterility in the next back-cross to guinea-pigs. If the two classes of females occurred in about equal frequency (as one would expect) then 75 per cent of the $\frac{1}{8}$ wild males would be fertile. Expressing this mating in the usual terms, it would read as follows:

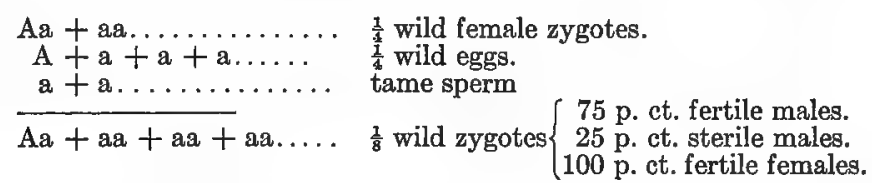

Now, if the numbers were large, and the different zygotic classes of $\frac{1}{8}$ wild females were represented in the expected proportions, then seven-eighths or 87.5 per cent of the $\frac{1}{16}$ wild males should be fertile. In any generation $\frac{2^{n-1}-1}{2^{n-1}}$ males should be fertile ( $\mathrm{n}$ being the number of the hybrid generation).

Table 77 gives the probable percentages of fertile males expected in each generation from the $F_{1}$ to the $F_{9}$ inclusive, it being supposed that very large numbers are involved and that the females of any generation are distributed approximately in the expected proportions of the different zygotic classes. Our actual experimental data show that the case is far from being as simple as this, for the percentage of fertile males in each generation does not agree with the series expected on the basis of one factor as given in table 77. Furthermore, on the basis of one factor, the males would also be divided into two distinct classes: sterile (Aa) and fertile (aa). It was shown that this was not the case. The hypothesis, at least in this simple form, does not agree with the facts.

Now, if the sterility of the males had been due to disturbances between either one or both of two Mendelian pairs of factors or pairs of homologous chromosomes, then we should have expected one-fourth of the $\mathrm{F}_{2} \frac{1}{4}$ wild males to be fertile. If we represent the two factors from the wild as $A$ and $B$, and the two from the tame as a and $b$, then the mating of the wild, $\mathrm{AABB}$, with the tame, aabb, would give hetero- 
zygotes $\mathrm{AaBb}$. The females would be unaffected, but the males would be sterile on account of the disturbances between $A$ and $a$, and between $B$ and $b$. Mating the fertile females, which likewise have the zygotic formula $\mathrm{AaBb}$, back to the guinea-pig, aabb, would give the following:

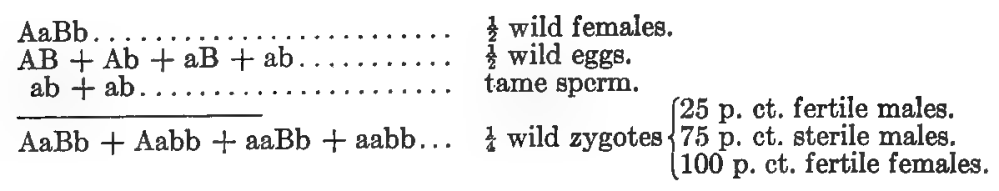

This hypothesis would explain the absolute sterility of some $\frac{1}{4}$ wild males $(\mathrm{AaBb})$, but also admit of a further maturation or tendency to fertility in those individuals with less disturbing combinations, $i$. e., with more factors from the tame (Aabb and $\mathrm{aaBb}$ ). The ultimate recessive, aabb, would be fertile and would occur in 25 per cent of the cases. Now, if the numbers were large and the different zygotic classes of $\frac{1}{4}$ wild females were represented in about the expected proportions given, then 56.25 per cent of the $F_{3}$, or $\frac{1}{8}$ wild males, would be fertile. One could not distinguish the different classes of $F_{2}$ females by inspection, but the random mating to guinea-pig males would be symbolized as follows:

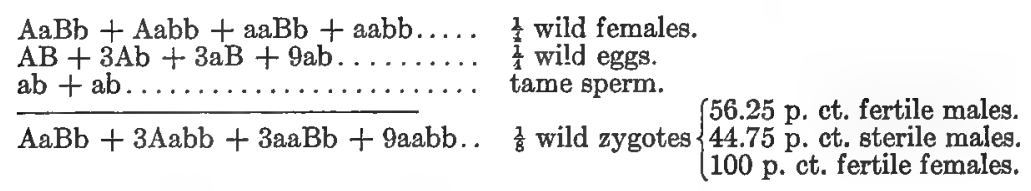

Here again, if the numbers were large and the different zygotic classes of females were represented in the expected proportions, then 76.56 per cent of the $\frac{1}{16}$ wild males should be fertile; and, in any generation, $\left(\frac{2^{n-1}-1}{2^{n-1}}\right)^{2}$ males should be fertile ( $\mathrm{n}$ being the number of the hybrid generation).

Table 77 likewise gives the most probable percentages of fertile males expected in each generation from the $F_{1}$ through the $F_{9}$ on the basis of two factors, it being supposed that the females of any generation are distributed in the expected proportions of the different zygotic classes.

The most probable percentages of fertile males, the ultimate recessives in the different generations on the basis of various numbers of factors, from 1 to 9 , are given in table 77. The general scheme will be evident from an examination of this table, for, stated in simple manner, the percentages of fertile males would be as given in table $\mathrm{A}$. 
TABLE A.

\begin{tabular}{|c|c|c|c|c|}
\hline $\begin{array}{c}\text { Hybrid } \\
\text { generation. }\end{array}$ & With 1 factor. & With 2 factors. & With 3 factors. & With $\mathrm{p}$ factors. \\
\hline $\begin{array}{l}\mathbf{F}_{2} \ldots \ldots \\
\mathbf{F}_{3} \ldots \ldots \\
\mathbf{F}_{4} \ldots \ldots \\
F_{n} \ldots \ldots\end{array}$ & $\begin{array}{l}.50 \text { fertile. } \\
.75 \text { fertile. } \\
.875 \text { fertile. } \\
\frac{2^{n-1}-1}{2^{n-1}} \text { fertile. }\end{array}$ & $\begin{array}{l}.25 \text { fertile. } \\
.5625 \text { fertile. } \\
.7656 \text { fertile. } \\
\left(\frac{2^{n-1}-1}{2^{n-1}}\right)^{2} \text { fertile. }\end{array}$ & $\begin{array}{l}(.50)^{3} \text { fertile. } \\
(.75)^{3} \text { fertile. } \\
(.875)^{3} \text { fertile. } \\
\left(\frac{2^{n-1}-1}{2^{n-1}}\right)^{3} \text { fertile. }\end{array}$ & $\begin{array}{l}(.50)^{\mathrm{p}} \text { fertile. } \\
(.75)^{\mathrm{p}} \text { fertile. } \\
(.875)^{\mathrm{p}} \text { fertile. } \\
\left(\frac{2^{\mathrm{n}-1}-1}{2^{\mathrm{n}-1}}\right)^{\mathrm{p}} \text { fertile. }\end{array}$ \\
\hline
\end{tabular}

From these series we may say that in any given generation, $F_{n}$, in which the degree of wildness is $\frac{1}{2^{n}}$, the number of fertile males should be $\left(\frac{2^{n-1}-1}{2^{n-1}}\right)^{n}$, where $n$ equals the number of the hybrid generation and $p$ equals the number of factors. In actual breeding experiments the chances of error would be great. To realize such a series of segregates, the different classes of females of each generation would also have to occur in approximately the expected proportions in order to give the expected percentage of ultimate recessive males in the next generation. This could only be accomplished by raising very large numbers.

It is quite impossible to determine from our data whether or not the percentage of fertile males in each generation corresponds in any measure to a theoretical percentage which is based on a definite number of factors; because, as tables 72 to 75 show, not all males with many motile sperm could be tested also by breeding. Furthermore, it is shown in table 76 that at least 10 per cent of the males whose microscopic test gave every promise of being fertile were actually sterile after a rigid breeding test. We may feel more confident of the proportions with many motile sperm than of the proportions really fertile. If we examine the percentage of males in each hybrid generation, the contents of whose epididymis could not be distinguished from that of a normal guinea-pig, we find (see table 75) the following series of percentages from the $F_{1}$ to the $F_{7}$ inclusive:
0.00
0.00
14.29
33.33
60.67
69.39
73.33

If we take the percentage of males with many motile sperm in the total tested by all methods the series is about the same:
0.00
0.00
9.46
32.38
60.67
69.39
73.33

This latter series of percentages imputes that all males sterile in a thorough breeding test alone did not have many motile sperm. From table 76 we see that this is not completely true in about one-tenth of the cases. The first series is probably more accurate, as it is the percentage of males with many motile sperm in the total of microseopic 
tests rather than in the total of all tests. The series of percentages of ultimate recessives expected on the basis of eight factors (see table 77) is:
0.00
$0.39 \quad 10.01$
34.36
59.67
77.57
88.16

One must admit that there is a remarkable similarity between these three series for the first 5 hybrid generations at least-such a close resemblance that one wonders whether it is chance coincidence or whether there actually were 8 allelomorphic pairs involved, such that the ultimate recessives in each generation segregate out with many motile sperm. One would be forced to conclude that further factors were necessary to give real fertility in addition to mere numbers and motility, for it was shown that males with many motile sperm were not necessarily fertile. The great range of possibilities between no sperm and all motile sperm would, on this hypothesis, be due to recombinations of factors. Individuals homozygous in 6 or 7 recessive factors would be almost fertile, for they would have segregated out most of the disturbing "wild chromosomes" and have replaced them with homologous pairs entirely from the tame source.

Such an hypothesis is suggestive and alluring, but other critical considerations are necessary. The probable errors for the percentages were calculated, but are not given. I am indebted to Dr. H. L. Rietz for valuable suggestions regarding these. They would be extremely difficult to handle and very misleading. The probable error of any generation would have to be calculated on the supposition that the females of the preceding generation were normally distributed, or else one would have to take the error of all preceding generations into account. It is logically impossible to suppose that the females of any generation (except $\mathrm{F}_{1}$ ) could have been normally distributed. On this hypothesis we would suppose that the wild and tame had 8 factors or chromosomes which were incompatible in the $F_{1}$ males, and this led to disturbances in the maturation of the sperm, but did not affect the females. We might represent the factors from the wild as AABBCCDDEEFFGGHH, and those from the tame as aabbceddeeffgghh. The $\frac{1}{2}$ wild would be Aa Bb Cc Dd Ee Ff Gg Hh. The fertile $F_{1}$ females should then produce 256 kinds of gametes, but only one of these, abcdefgh, would have segregated out the disturbing elements from the wild. Now, when this gamete met its mate from the tame, also abcdefgh, it should have given fertility in the $\mathrm{F}_{2}$ males. But the expectation of this combination based on random sampling is 1 in 256 . The number of $\mathrm{F}_{2}$ males (22) actually procured was far too small to expect an ultimate fertile recessive male. One would, however, expect recombinations which had eliminated some of the disturbing elements. Such were actually obtained, for $2 \mathrm{~F}_{2}$ males showed a few deformed, immotile sperm. (See tables 74, 75.) If the ultimate recessive, fertile males actually lacked all disturbing elements from the wild, then in 
mating them to the tame guinea-pigs we should expect them to breed true to fertility on this hypothesis. In spite of hypotheses, when fertile males occurred and were bred to guinea-pigs the male offspring were not all completely fertile, as will be shown. Hence we can not regard the fertile males as simple, ultimate recessives in a Mendelian sense. There is evidence of segregation of factors for fertility, but the case is more complicated than the strict hypothesis of 8 factors allows. ${ }^{1}$ What part interaction of factors plays, we do not know. Nor do we know that all guinea-pigs carry the absence of factors disturbing fertility in these crosses.

It may be added that some definite characters from the wild were surely compatible with fertility, because males with the "wild agouti" were also fertile.

THE MALE OFFSPRING OF FERTILE MALE HYBRIDS.

Offspring of fertile male hybrids were also tested. They may be divided into two classes: the offspring of fertile male hybrids and female hybrids, and the offspring of fertile male hybrids and guineapigs. It seems that when male hybrids were fertile they could be bred to any sort of fertile female (see table 78). Male guinea-pigs have been bred to all classes of female hybrids from the $F_{1}$ to the $F_{9}$ generation inclusive. Male hybrids of every class from the $\mathrm{F}_{3}$ through $\mathrm{F}_{6}$ were bred successfully to guinea-pig females. Male hybrids in each generation from the $F_{3}$ to the $F_{7}$ inclusive were successful sires in matings with female hybrids of the same or different generations. In this last class of matings such diverse crosses as the following were possible: $F_{5}$ males were bred to $F_{1}, F_{4}, F_{5}$, and $F_{6}$ female hybrids, while $F_{6}$ males were bred to $F_{2}, F_{5}, F_{6}$, and $F_{7}$ females. A $\frac{1}{2}$ wild female, 5 years old, was impregnated by her great great grandson. A $\frac{1}{4}$ wild female was successfully mated with a $\frac{1}{64}$ wild male. The different possible combinations of successful matings indicate that fertile male hybrids of any blood dilution can impregnate any sort of fertile female.

Fertili Male Hybrids in Crosses with Female Hybrids.

In all, 39 offspring from this sort of mating were tested (see table 72); 36 received only a microscopic test, while 3 received both tests. Tables 74 and 76 show that all classes of males were produced, ranging

${ }_{1}$ The percentages of males with many motile sperm in the $F_{6}$ and $F_{7}$ generations were 69.4 per cent and 73.3 per cent respectively (table 75). As a matter of fact, these percentages do not correspond to the expectatinns based on 8 factors (table 77), but are nearer the results one would expect with 12 or 20 factors in the $F_{6}$ and $F_{7}$ generations respectively. This can be readily computed from the formula given on page 95 .

$$
\begin{aligned}
& F_{6} \ldots\left\{\frac{2^{n-1}-1}{2^{n-1}}\right\}^{p}=.694 \quad(.96875)^{p}=.694 \quad p \log .96875=\log .694 \quad p=11.51 \\
& F_{7} \ldots \ldots \ldots(.98438)^{p}=.733 \quad p \log .98438=\log .733 \quad p=19.72
\end{aligned}
$$


from those with no sperm to those fertile in breeding. The $\frac{1}{8}$ wild males, bred to $\frac{1}{8}$ wild females, gave one male with many motile sperm (fertile in breeding also) out of 7 tested. The $\frac{1}{16}$ wild males, bred to their sisters in blood, gave 8 males with many motile sperm out of 14 tested. The $F_{5}$ males bred to $F_{5}$ females gave 5 males with many motile sperm out of 8 tested. One $F_{7}$ male bred to an $F_{7}$ female gave one male, and he had many motile sperm. The other 9 matings correspond to these, for irrespective of what generation the fertile male sires were they gave a preponderance of sterile male offspring when bred to intense wild-blooded female hybrids, but increasing signs of fertility in their sons when bred to females of later generations. For example, the $F_{5}$ and $F_{6}$ males, bred to $F_{1}$ and $F_{2}$ females respectively, gave entirely sterile sons; but one $\mathbf{F}_{5}$ male gave sons with many motile sperm when mated to $\mathrm{F}_{4}$ females, while two $\mathrm{F}_{4}$ males, bred to $\mathrm{F}_{5}$ females, gave sons with many motile sperm also.

If the hypothesis advanced is correct, and a fertile male hybrid represented the same combination of factors for fertility as a guineapig male, then from mating fertile male hybrids with female hybrids we should expect about the same results that were obtained by mating guinea-pigs to similar female hybrids. We have already shown that when guinea-pigss were mated to the different generations of female hybrids, increasing signs of fertile males came with each back-cross. The hypothesis implies that more and more females were being obtained which lacked the disturbing factors and failed to transmit such. The results in the sons of fertile male hybrids bred to female hybrids are consistent with this hypothesis, for the intense wild females gave more sterile sons than the dilute wild females in this class of matings, just as they did when mated to guinea-pigs. The two series of percentages of males with many motile sperm produced in these matings are given in table 79. The number of sons from female hybrids and male hybrids is far too small for broad generalizations; but the results indicate that sterility is transmitted in the same manner by the female hybrids crossed with male hybrids as when crossed with guineapigs. The percentages of sons with many motile sperm in both sorts of crosses in the different generations from the $F_{1}$ to the $F_{7}$ are as follows:

$\begin{array}{lllllll}0.0 & 0.0 & 14.3 & 58.8 & 63.6 & \ldots & 100.0 \text { with fertile hybrid sires. }\end{array}$

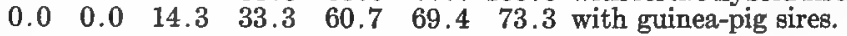

For further details and numbers involved, see table 79 .

Fertile Hybrid Males in Crosses with Guinea-pigs.

A total of 22 sons from this sort of mating was tested, all having a microscopic test only. The fertile hybrid sires belonged to the $F_{3}, F_{4}$, $\mathrm{F}_{5}$, and $\mathrm{F}_{6}$ generations (see tables 72 to 76 ). To test the hypothesis that the fertile hybrid sires, with many motile sperm, had segregated 
out as recessives and that we should expect the same results from such fertile hybrid males as with guinea-pig males, 14 of them were mated with guinea-pig females. The microscopic tests showed that 21 of their 22 sons were indistinguishable from a normal guinea-pig male. The one exception ( $\sigma^{7} 1524$ ) was the son of an $\mathrm{F}_{4}$ male $\left(\sigma^{7} 506\right)$ and a guinea-pig female ( $(186)$. The same sire and dam gave two other sons ( $o^{15}$ and $o^{3} 16$ ) with many motile sperm. The exceptional son had nothing except motile sperm in the epididymis, but they were extremely few in number. I am informed by Dr. W. E. Castle that other sons of fertile hybrid males and guinea-pig females likewise showed signs of sterility. Fertility, however, appears to be obtained most frequently from this class of matings, as the records show that 95.5 per cent of the sons of fertile hybrid males and guinea-pigs had many motile sperm. In view of these facts, the hypothesis (that fertility in the hybrids simply means eliminating 8 disturbing factors) can not be maintained. There is strong evidence of segregation, but the case may be complicated by other conditions, such as interaction of factors and the like.

\section{THE SECONDARY SEXUAL CHARACTERS.}

Some observations on the secondary sexual characters were made. Although not taken as statistical data, they were numerous enough to be of value. Male hybrids of all classes showed the sex instinct. In fact, I have never seen a single healthy male hybrid, sterile or fertile, which did not attempt copulation. The hybrids fought with each other for the possession of the females. How successful they were in copulation is not known, but since the organs were morphologically similar to those of a normal guinea-pig, it is probable that there were no difficulties in this respect at least. There is good evidence that ejaculation took place and that normal uterine plugs were formed from the clotted mass, as in the case of any normal guinea-pig. It is well known that severing the spinal cord will often produce an ejaculation. The method of killing the hybrids was to sever the skull and axis by holding the head, swinging the animal and suddenly arresting the motion. It was. noticed that in all cases an ejaculation took place if one then pressed the groin, a clot forming almost immediately. This clotting or coagulation of the semen, supposed to be due to a ferment, vesiculase (Marshall 1910), is common to both the hybrid and the guinea-pig males and gives rise to the uterine plug in the female. More than 200 hybrid males: showed this peculiar reaction when properly stimulated. No hybrids failed to show it if they were killed when adult. Hence it is almost certain that they were physiologically potent in every respect, except in the production of sperm. The accessory organs, including the seminal vesicles and prostate glands, were always apparently normal. The only differences noted were that sterile hybrids might have small testes and a pale, small epididymis. 


\section{THE FECUNDITY OF THE FEMALE HYBRIDS.}

Almost every female hybrid in each generation was fertile in breeding. The only exceptional generation was the $\frac{3}{4}$ wild, in which the only female was sterile. Occasionally a female hybrid was sterile, but such cases were infrequent. Although no data were taken on sterility in guinea-pigs, I am of the opinion that sterility in the female hybrids was no more frequent than in these. There was at least one source of data which gave information on the degree of fecundity in the female hybrids - the average number of young per litter (see table 80).

The wild $C$. rufescens, bred in captivity, gave 46 offspring in 34 litters, or an average of 1.35 per litter. We do not know what their average per litter is in the wild habitat. The tame guinea-pigs, used as dams in matings with wild sires to produce the $\frac{1}{2}$ wild hybrids, gave 37 young in 16 litters, or an average of 2.31 per litter. This shows that the wild males impregnated the guinea-pigs just as successfully as a guinea-pig male would have; for the average per litter in our guinea-pigs was 2.34. Minot (1891) found an average of 2.56 in his experiments with tame guinea-pigs, but his numbers were smaller (see table 80).

The $\mathrm{F}_{1}$ hybrids were intermediate, for they produced 83 young in 52 litters, or an average of 1.60. In fact, they were a little less fecund than a theoretical midparental condition would demand, for this would be 1.845. They were about as "wild," to all appearances, as the pure wild females, but were slightly more prolific. The $\mathrm{F}_{2}$ hybrid females, the $\frac{1}{4}$ wild, produced 217 young in 114 litters, or an average of 1.90 per litter. The $\mathrm{F}_{3}$ hybrid females produced 312 offspring in 152 litters, or an average of 2.05. The subsequent hybrid generations did not show an increased average, although they were produced by successive backcrosses to the guinea-pig male.

The analysis of these data is complicated by a number of conditions. The guinea-pigs raised in our laboratory gave larger litters in summer than in winter; for in summer they produced 218 young in 85 litters, or an average of 2.56 , whereas in winter they produced 266 young in 122 litters, an average of 2.16 per litter. The young born from January 15 to July 15 were considered winter litters in these data, because the ovulations and conceptions corresponding to these births ranged from about November 8 to May 8. Minot (1891) found a similar condition in his experiments.

Minot also found that the first litters were smaller than the average; but first litters are usually borne by young females and it may mean that the smallness of first litters is entirely an effect of age. This may account for the fact that our $\mathrm{F}_{4}, \mathrm{~F}_{5}$, and $\mathrm{F}_{6}$ females failed to show an increased average per litter, since many of the female hybrids in these generations were young, and the records contain a large proportion of litters from such females. 
The results therefore show that in mating the wild C. rufescens to guinea-pigs, the litter average of the $\mathrm{F}_{1}$ hybrids was about intermediate, and continued back-crosses raised this average gradually.

It may be added that the proportion of females producing some fertile males or males with all evidences of fertility gradually increased in each generation. Certain females in the later generations produced only fertile males, but the number of young from one female was necessarily small and we can not be sure but that they would have given sterile sons had larger numbers been possible. However, one should eventually be able to produce female hybrids with the fecundity of the guinea-pig species and having only fertile sons. Combining these characters with wild characters, such as the peculiar wild agouti, should also be possible.

Two abnormal females ( $\$ 263 \frac{1}{4}$ wild and $\$ 393 \frac{1}{16}$ wild) should be recorded. The former, $\& 263$, had an enlarged clitoris, resembling a penis, but also all the female characters, bore 2 young, and gave milk. It was difficult to keep her with a male, for as she grew older they fought continually. The latter, o 393 , also had an enlarged clitoris, which was very nearly of the same form and size as a normal penis. The female external characters were all normal. She had no young to my knowledge, but upon one occasion she showed large and abrupt loss of weight, and gave milk at the same time. It is barely possible that she had aborted. When kept alone for some time, and subsequently placed with a male, she allowed the male to attempt copulation. When placed with females she always attempted copulation, making the same sounds and going through the movements of a normal male. If she was penned with a male and females, she and the male fought continually for the possession of the females. She was killed at the age of 2 years, and the ovaries were examined. They were abnormally large, measuring about $1 \frac{1}{2}$ inches in length and an inch in width. The follicles were greatly distended, some measuring 0.75 inch in diameter.

Abnormal ovaries of this type were not uncommon in other female hybrids which bore young and were otherwise perfectly normal in all respects. The viscera of many female hybrids were examined, but no data were taken on the occurrence of this type of abnormality.

\section{THE SEX RATIO IN THE HYBRIDS.}

The many recent experiments with sex-linked and sex-repelled characters have led to the current opinion that sex itself is a Mendelian character, and that one sex is homozygous while the other is heterozygous for sex-determining factors. One would expect an equality of the two sexes in the long run on this hypothesis; but when an excess of one sex occurs consistently, it is supposed that the heterozygous parent fails to produce the two kinds of gametes in equal numbers, or 
that selective fertilization takes place, or that unequal viability of the two sexes during early development accounts for the discrepancies.

Guyer (1909), compiling the proportion of sexes in hybrid birds, stated: "When due allowance is made for all errors, the facts still indicate that there is a marked tendency for the hybrids, especially those from widely separated parents, to be male." Since the female is supposed to be favored by increased nutrition, he thought the excess of males might be due to default in metabolic processes because of incompatibilities between dissimilar germ plasms, such incompatibilities being especially inimical to the production of females.

King (1911), tabulating the sex ratios of hybrids between wild and albino rats, stated: "It appears, therefore, that hybridizing alters the sex ratio by producing a marked increase in the relative proportion of males. This conclusion is in essential agreement with that reached by Buffon, by R. and M. Pearl, and by Guyer."

King found 231 males to 194 females in the totals of the first three hybrid generations, this being a ratio of 119.07 males to 100 females.

Minot (1891) crossed guinea-pigs inter se and obtained 223 males to 187 females, or a ratio of 119.2 males to 100 females.

The results in the hybrids between $C$. rufescens and C. porcellus did not show an excess of males, but, to the contrary, a significant excess of females (see table 81). The wild parent bred in captivity gave 20 males, 25 females, and 1 of unknown sex. The $\frac{1}{2}$ wild hybrids gave 14 males and 23 females, or a ratio of 60.87 males to 100 females. There were 2 young of unknown sex, having died prematurely. If we call them males, the ratio is 69.57 males to 100 females. The $\mathrm{F}_{2}$, or $\frac{1}{4}$ wild, gave 31 males and 52 females, or a ratio of 59.62 males to 100 females. The $\mathrm{F}_{3}$, or $\frac{1}{8}$ wild, gave 101 males to 116 females, or a ratio of 87.07 males to 100 females. It is apparent that, as the generations became less hybrid in nature, the sexes were gradually approaching equality.

After the $\frac{1}{8}$ wild, the sexes were more nearly equal, for the next four generations gave a total of 406 males to 409 females, practically an equality of sexes, for the ratio is 99.24 males to 100 females. This is strikingly different from the total of the first three generations, in which there were 146 males to 191 females, or a ratio of 76.44 males to 100 females. The total results of all hybrids were 552 males and 600 females, or a ratio of 92 males to 100 females. These ratios do not confirm the results shown by Guyer or King.

Previous data have shown that sterility was common in the males of the early hybrid generations, for there were disturbances in spermatogenesis. It is shown here that the early generations also gave a deficiency of males. May it not be possible that the same incompatibilities between dissimilar germ plasms which gave rise to sterility in gametogenesis also caused disturbances in fertilization. Possibly male 
zygotes may have been formed less frequently, or may have been eliminated at an early stage.

The only similar case in mammalian crosses which I have been able to find is that described by Boyd (1914), in which the bison and domestic cattle were crossed. Boyd found that his hybrids gave 60 females to 17 males, or a ratio of 28.33 males to 100 females. Boyd likewise found sterility common in the males, similar to that in my hybrids.

\section{SUMMARY AND GENERAL CONCLUSIONS.}

(1) Crosses between C.rufescens males and C.porcellus females gave completely sterile male hybrids and fertile female hybrids. By crossing the female hybrids back to guinea-pig males, $\frac{1}{4}$ wild hybrids were obtained, which were again sterile males and fertile females. A few males of this second hybrid generation, however, showed some degenerate non-motile sperm. By repeated back-crosses of female hybrids to guinea-pigs, increasing signs of fertility appeared. Fertility seemed to act like a very complex recessive character; for the results obtained were what one would expect if a number of dominant factors for sterility were involved, the elimination of which would give a recessive fertile type. There was an enormous range of forms between hybrids with no sperm and fertile hybrids with many motile sperm.

(2) The results indicated that a completely fertile hybrid male could be bred to female hybrids or to guinea-pigs, giving about the same results as a normal guinea-pig male in such matings.

(3) The secondary sexual characters of all malehybrids were normally developed.

(4) The wild C.rufescens has a smaller litter average than the guineapig. When the wild males were bred to guinea-pig females, the size of the litters was that of the guinea-pig. The female hybrids produced by this cross, however, gave a litter average intermediate between that of the wild and tame. By repeatedly crossing the hybrid females of one generation back to guinea-pig males to produce the next hybrid generation, the litter average was raised almost to that of the guineapig itself. This is all the more interesting since guinea-pig males were used to raise the litter average.

(5) Two female hybrids showed some male secondary sexual characters. One of these with marked male instincts had abnormal ovaries. Abnormal ovaries were common in the female hybrids.

(6) The sex ratio in the hybrids showed a marked preponderance of females, expecially in the early hybrid generations, $i . e$., in those generations which must have been most hybrid in constitution. 


\section{T A B LES.}

TABLE 1.

Matings of wild females with wild males, all the offspring like the parents showing the agouti pattern.

\begin{tabular}{|c|c|c|}
\hline \multicolumn{2}{|c|}{ Parents. } & Offspring. \\
\hline \multicolumn{2}{|c|}{$\% \mathrm{AA} \times \sigma^{\top} \mathrm{AA}$} & $\mathrm{AA}$ \\
\hline 2 & 1 & 3 \\
\hline 3 & 1 & 7 \\
\hline 4 & 1 & 2 \\
\hline 15 & 33 & 3 \\
\hline 25 & 1 & 7 \\
\hline 46 & 1 & 6 \\
\hline 46 & 55 & 3 \\
\hline * $\left\{\begin{array}{l}3 \\
4\end{array}\right.$ & 1 & 1 \\
\hline$\left\{\begin{array}{l}4 \\
2\end{array}\right.$ & $\begin{array}{l}1 \\
\ldots\end{array}$ & 1 \\
\hline 3 & .. & $\therefore$ \\
\hline 4 & 1 & \\
\hline 15 & 24 & 7 \\
\hline 2 & .. & .. \\
\hline $\begin{array}{l}3 \\
4\end{array}$ & $\cdots$ & . \\
\hline$\left\{\begin{array}{r}4 \\
15\end{array}\right.$ & $\because$ & $\because$ \\
\hline 25 & 1 & 1 \\
\hline 2 & - & $\cdots$ \\
\hline $\begin{array}{l}3 \\
4\end{array}$ & $\ddot{i}$ & $\cdots$ \\
\hline 15 & 24 & $\because$ \\
\hline 25 & 33 & 6 \\
\hline \multicolumn{2}{|c|}{ Total..... } & 46 \\
\hline
\end{tabular}

*In this and the subsequent matings an uncertainty exists as to the identity of one or both parents. The record here given indicates all the possibilities, based on the record of what animals were penned together.

TABLE 4.

Matings of ${ }_{1 \overline{8}}^{1}$ wild, homozygous in agouti, with guinea-pigs lacking it.

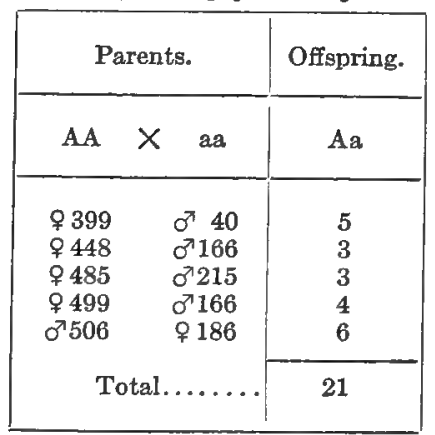

104
TABLe 2.

Matings of non-agouti guinea-pig females to wild agouti males, producing heterozygous agouti young.

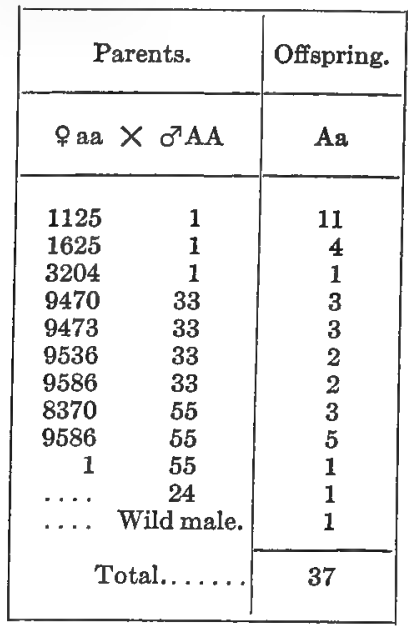

TABLe 3.

Matings of $\frac{2}{\mathrm{n}}$ wild females, heterozygous in or lacking agouti, with guinea-pig males homozygous in agouti. Offspring, all agouti.

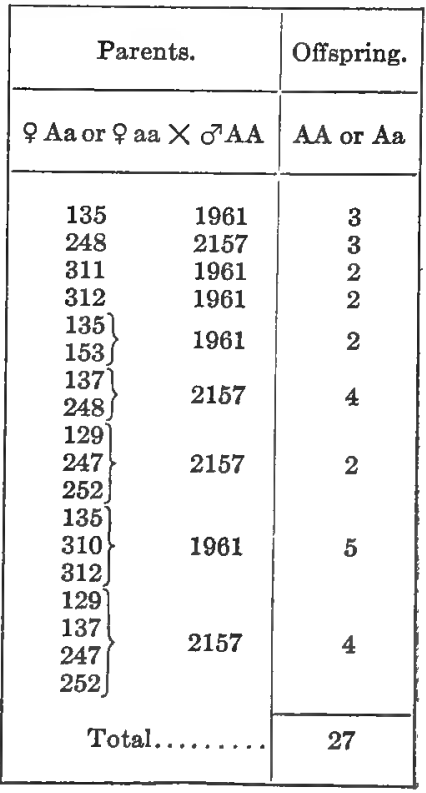


TABLES.

TABle 5.

Summary of Tables 2,3 , and 4, showing agouti always epistatic.

\begin{tabular}{|c|c|c|c|c|c|}
\hline \multirow{2}{*}{ Table. } & \multicolumn{4}{|c|}{ Parents. } & \multirow{2}{*}{$\frac{\text { Offspring }}{\text { Agouti. }}$} \\
\hline & Females. & Color. & Males. & Color. & \\
\hline 2 & Guinea-pig. & as & Pure wild. . & AA & 37 \\
\hline 3 & $\frac{1}{8}$ wild. . & aa & Guinea-pig & AA & 27 \\
\hline \multirow[t]{2}{*}{4} & $\frac{1}{16}$ wild .... & AA & Guivea-pig & aa & 15 \\
\hline & Total & & $\ldots \ldots \ldots$ & & 85 \\
\hline
\end{tabular}

TABLE 7.

TABle 6.

Matings of 1 wild females, heterozygous in agouti, with guinea-pig males lacking it.

Matings of $\frac{1}{2}$ wild females, heterozygous in agouti, with guinea-pig males lacking it.

\begin{tabular}{|c|c|c|c|}
\hline \multicolumn{2}{|c|}{ Parents. } & \multicolumn{2}{|c|}{ Offspring } \\
\hline \multicolumn{2}{|c|}{ OAa $\times \sigma^{7} \mathrm{aa}$} & \multicolumn{2}{|c|}{$\mathrm{Aa}+\mathrm{aa}$} \\
\hline 63 & 11030 & 2 & $\mathbf{5}$ \\
\hline 63 & 2193 & 3 & 1 \\
\hline 68 & 11030 & 6 & 4 \\
\hline 68 & 2193 & 0 & 2 \\
\hline 68 & 4 & 3 & 2 \\
\hline 69 & 11030 & 3 & 3 \\
\hline 69 & 4 & 0 & 2 \\
\hline 72 & 617 & 0 & 4 \\
\hline 75 & 9246 & 2 & 0 \\
\hline 75 & $\mathrm{NW}$ & 2 & 0 \\
\hline 118 & 11030 & 3 & 1 \\
\hline 253 & G & 3 & 0 \\
\hline 253 & 617 & 0 & 1 \\
\hline $\left.\begin{array}{r}69 \\
118\end{array}\right\}$ & 11030 & 1 & 1 \\
\hline $\left.\begin{array}{r}69 \\
118\end{array}\right\}$ & 2193 & 3 & 1 \\
\hline$\left.\underset{\frac{1}{2} \text { wild }}{75}\right\}$ & 9246 & 2 & 1 \\
\hline$\frac{1}{2}$ wild & 9246 & 6 & 6 \\
\hline$\frac{1}{2}$ wild & 11030 & 8 & 2 \\
\hline & otal.... & 47 & 36 \\
\hline $\begin{array}{r}\text { Most } p \\
\text { expec }\end{array}$ & $\begin{array}{l}\text { robable } \\
\text { tation... }\end{array}$ & $\left\{\begin{array}{l}42 \\
41\end{array}\right.$ & $\begin{array}{l}41 \\
42\end{array}$ \\
\hline
\end{tabular}

\begin{tabular}{|c|c|c|c|}
\hline \multicolumn{2}{|c|}{ Parents. } & \multirow{2}{*}{\multicolumn{2}{|c|}{$\frac{\text { Offispring. }}{\mathrm{Aa}+\mathrm{aa}}$}} \\
\hline \multicolumn{2}{|c|}{ OAa $\times \sigma^{\sigma^{7}} \mathrm{aa}$} & & \\
\hline 87 & 170 & 1 & 1 \\
\hline 90 & 1541 & 3 & 6 \\
\hline 90 & 214 & 2 & 1 \\
\hline 91 & 1541 & 4 & 3 \\
\hline 91 & 214 & 2 & 1 \\
\hline 92 & 9246 & 1 & 0 \\
\hline 92 & 1541 & 1 & 1 \\
\hline 95 & 1541 & 1 & $\mathbf{0}$ \\
\hline 96 & 12612 & 4 & 8 \\
\hline 96 & 170 & 1 & 1 \\
\hline 97 & 11030 & 0 & 1 \\
\hline 98 & 12612 & 0 & 2 \\
\hline-105 & 1541 & 6 & 3 \\
\hline 106 & 0 & 0 & 1 \\
\hline 107 & 9758 & 2 & 1 \\
\hline 107 & 617 & 1 & 1 \\
\hline 107 & -25 & 1 & 2 \\
\hline 107 & 199 & $\hat{I}$ & 0 \\
\hline 122 & 12612 & 2 & 1 \\
\hline 147 & 1543 & $\overline{1}$ & 1 \\
\hline 149 & G & 1 & 1 \\
\hline 150 & 1543 & B & 2 \\
\hline 150 & 201 & 0 & 1 \\
\hline 160 & 617 & 1 & i \\
\hline 160 & -25 & 1 & 3 \\
\hline 550 & 199 & 1 & 1 \\
\hline 606 & 201 & $\hat{1}$ & 2 \\
\hline 645 & 199 & 1 & 1 \\
\hline 90 ? & & & \\
\hline 95$\}$ & 1541 & 2 & 2 \\
\hline 105 & & & \\
\hline $\begin{array}{r}91 \\
1\end{array}$ & 1541 & 2 & 1 \\
\hline $\begin{array}{r}106 \\
98\end{array}$ & & & \\
\hline 122$\}$ & 12612 & 2 & 4 \\
\hline $\begin{array}{r}06 \\
122\end{array}$ & 12612 & 1 & 4 \\
\hline 92$\}$ & 1541 & 2 & 1 \\
\hline & Total... & 55 & 59 \\
\hline $\begin{array}{r}\text { Mos } \\
\text { ex }\end{array}$ & probable & 57 & 57 \\
\hline
\end{tabular}


Table 8.

Matings of $\frac{1}{6}$ wild females with guinea-pig males, in which one parent is heterozygous in agouti and the other lacks it entirely.

\begin{tabular}{|c|c|c|c|}
\hline \multicolumn{2}{|c|}{ Parents. } & \multicolumn{2}{|c|}{ Offspring. } \\
\hline \multicolumn{2}{|c|}{ OAa $\times$ o $^{7} \mathrm{aa}$} & \multicolumn{2}{|c|}{$\mathrm{Aa}+\mathrm{aa}$} \\
\hline 140 & 1881 & 5 & 1 \\
\hline 166 & 85 & 0 & 1 \\
\hline 238 & 2366 & 0 & 3 \\
\hline 311 & 2278 & 2 & $\mathbf{0}$ \\
\hline 470 & 2036 & 2 & 0 \\
\hline 599 & 2278 & 2 & 3 \\
\hline 723 & 117 & & 0 \\
\hline \multicolumn{2}{|c|}{ o aa $\times \sigma^{7} \mathrm{Aa}$} & \multicolumn{2}{|c|}{$\mathrm{Aa}+\mathbf{a} \mathbf{a}$} \\
\hline 141 & 2002 & 0 & 1 \\
\hline 143 & 2002 & 1 & 0 \\
\hline 144 & 2002 & 0 & 1 \\
\hline 145 & 2196 & 1 & 2 \\
\hline 170 & -30 & 1 & 2 \\
\hline 173 & 1917 & 5 & 0 \\
\hline 177 & 1923 & 1 & 0 \\
\hline 178 & 1923 & 7 & 5 \\
\hline 197 & -98 & 6 & 1 \\
\hline 205 & -98 & 0 & 4 \\
\hline 207 & 2083 & 1 & 2 \\
\hline 208 & 2083 & 1 & 4 \\
\hline 209 & 2083 & 2 & 1 \\
\hline 212 & 2083 & 1 & 0 \\
\hline 215 & -30 & 0 & 1 \\
\hline 234 & -30 & 2 & 1 \\
\hline 264 & 2002 & 4 & 1 \\
\hline 361 & 2196 & 1 & 0 \\
\hline 367 & 2196 & 1 & 1 \\
\hline \multirow{2}{*}{$\left.\begin{array}{l}209 \\
212\end{array}\right\}$} & 2083 & 3 & 2 \\
\hline & & & 37 \\
\hline \multirow{2}{*}{\multicolumn{2}{|c|}{$\begin{array}{c}\text { Total... } \\
\text { Most probable } \\
\text { expectation }\end{array}$}} & 50 & 37 \\
\hline & & $\left\{\begin{array}{l}43 \\
44\end{array}\right.$ & $\begin{array}{l}44 \\
43\end{array}$ \\
\hline
\end{tabular}

TABLE 10.

Matings of $\frac{1}{32}$ wild females, heterozygous in agouti, with guineapig males lacking it.

\begin{tabular}{|c|c|c|c|}
\hline \multicolumn{2}{|c|}{ Parents. } & \multicolumn{2}{|c|}{ Offspring. } \\
\hline \multicolumn{2}{|c|}{ OAa $Y \sigma^{7} \mathrm{aa}$} & \multicolumn{2}{|c|}{$\mathrm{Aa}+\mathrm{aa}$} \\
\hline 403 & 2278 & 1 & 0 \\
\hline 403 & 12835 & 3 & 0 \\
\hline 529 & 12835 & 6 & 5 \\
\hline 603 & 42 & 2 & 2 \\
\hline 687 & 115 & 2 & 1 \\
\hline 702 & 201 & I & 0 \\
\hline 733 & 201 & 2 & 1 \\
\hline 850 & 12835 & 0 & 1 \\
\hline \multicolumn{2}{|c|}{ Total. . . . } & 17 & 10 \\
\hline \multirow{2}{*}{\multicolumn{2}{|c|}{$\begin{array}{l}\text { Most probable } \\
\text { expectation. }\end{array}$}} & $\int 13$ & 14 \\
\hline & & 14 & 13 \\
\hline
\end{tabular}

Table 9.

Matings of $\frac{1}{18}$ wild females, heterozygous in agouti, with guinea-pig males lacking it.

\begin{tabular}{|c|c|c|c|}
\hline \multicolumn{2}{|c|}{ Parents. } & \multicolumn{2}{|c|}{ Offepring. } \\
\hline \multicolumn{2}{|c|}{ \% $\mathrm{Aa} \times \mathrm{d}^{7} \mathrm{aa}$} & \multicolumn{2}{|c|}{$A a+a a$} \\
\hline 195 & 2132 & 2 & 4 \\
\hline 277 & 2132 & 3 & 1 \\
\hline 277 & 72 & 1 & 4 \\
\hline 304 & 223 & 2 & 1 \\
\hline 317 & 163 & 0 & 1 \\
\hline 318 & 163 & 1 & 1 \\
\hline 340 & 12815 & 3 & 4 \\
\hline 341 & 12815 & 4 & 3 \\
\hline 356 & 223 & 2 & 2 \\
\hline 392 & 12815 & 3 & 3 \\
\hline 393 & 64 & 3 & 3 \\
\hline 414 & 40 & 3 & 3 \\
\hline 415 & 40 & 0 & 1 \\
\hline 416 & 40 & 1 & 0 \\
\hline 421 & 54 & 2 & 6 \\
\hline 435 & 166 & 0 & 4 \\
\hline 436 & 166 & 0 & 3 \\
\hline 461 & 217 & 2 & 1 \\
\hline 481 & 215 & 2 & 2 \\
\hline 519 & 144 & 2 & 3 \\
\hline 523 & 53 & 0 & 3 \\
\hline 536 & 64 & 2 & 0 \\
\hline 544 & 40 & 3 & 0 \\
\hline 556 & 104 & 1 & 2 \\
\hline 560 & 64 & 1 & 3 \\
\hline 565 & 64 & 2 & 3 \\
\hline 580 & 103 & 2 & 3 \\
\hline 601 & 103 & 1 & 0 \\
\hline 614 & 224 & 0 & 2 \\
\hline 2030 & 2006 & 2 & 2 \\
\hline 2030 & 2132 & 1 & 1 \\
\hline $\left.\begin{array}{l}304 \\
356\end{array}\right\}$ & 223 & 4 & 2 \\
\hline $\left.\begin{array}{l}277 \\
945\end{array}\right\}$ & 72 & 2 & 1 \\
\hline & otal..... & 57 & 72 \\
\hline Most & probable & $\{64$ & 65 \\
\hline exp & tation. . & 665 & 64 \\
\hline
\end{tabular}

TABLE 11.

Summary of Tables 6-10, in which an equality of agouti and non-agouti young is expected.

\begin{tabular}{|c|c|c|c|c|}
\hline \multirow{3}{*}{ 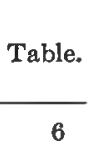 } & \multicolumn{2}{|c|}{ Parents. } & \multirow{2}{*}{\multicolumn{2}{|c|}{$\frac{\text { Offspring. }}{\mathrm{Aa}+\mathrm{aa}}$}} \\
\hline & \multirow{2}{*}{$\frac{\text { Females. }}{\frac{1}{2} \text { wild .... }}$} & \multirow{2}{*}{$\frac{\text { Males. }}{\text { Guinea pig. }}$} & & \\
\hline & & & 47 & 36 \\
\hline 7 & thild... & Guinea pig. & 55 & 59 \\
\hline 8 & $\frac{1}{8}$ wild.... & Guinea pig. & 50 & 37 \\
\hline 9 & $\frac{1}{18}$ wild .... & Guinea pig. & 57 & 72 \\
\hline 10 & $\frac{1}{32}$ wild.... & Guinea pig. & 17 & 10 \\
\hline \multicolumn{3}{|c|}{ 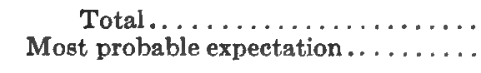 } & $\begin{array}{l}226 \\
220\end{array}$ & $\begin{array}{l}214 \\
220\end{array}$ \\
\hline
\end{tabular}


Tabie 12.

Matings of $\frac{1}{8}$ and $\frac{1}{16}$ wild females with guinea-pig males, both heterozygous in agouti.

\begin{tabular}{|c|c|c|c|}
\hline \multicolumn{2}{|c|}{ Parents. } & \multicolumn{2}{|c|}{ Offspring. } \\
\hline \multicolumn{2}{|c|}{$9 \mathrm{Aa} \times \sigma^{7} \mathrm{Aa}$} & \multicolumn{2}{|c|}{$\mathbf{A A}$ or $\mathbf{A a}+\mathbf{a a}$} \\
\hline $\begin{array}{c}\text { s wild. } \\
108 \\
108 \\
131 \\
166 \\
172 \\
198 \\
203 \\
219 \\
\frac{1}{16} \text { wild. } \\
536\end{array}$ & $\begin{array}{l}1436 \\
1917 \\
2196 \\
2196 \\
1917 \\
2002 \\
-98 \\
-98 \\
\\
1917\end{array}$ & $\begin{array}{l}4 \\
5 \\
1 \\
7 \\
3 \\
4 \\
1 \\
6\end{array}$ & $\begin{array}{l}0 \\
0 \\
0 \\
0 \\
0 \\
0 \\
0 \\
3\end{array}$ \\
\hline $\begin{array}{l}\text { Total } \\
\text { Most prol } \\
\text { pectatic }\end{array}$ & le $\begin{array}{l}\ldots \ldots \\
\ldots \ldots\end{array}$ & 27 & 4 \\
\hline
\end{tabular}

TABLE 13.

Offspring of female hybrids according to color of wild agouti.

\begin{tabular}{|c|c|c|c|c|}
\hline \multirow{2}{*}{ Mothers. } & \multicolumn{4}{|c|}{ Offspring. } \\
\hline & Ticked. & Dark. & Light. & $\begin{array}{l}\text { Per cent. } \\
\text { ticked. }\end{array}$ \\
\hline TICKED. & & & & \\
\hline$\frac{1}{2}$ wild . . . . & 18 & 5 & 19 & 43 \\
\hline wild ..... & 19 & 0 & 2 & 90 \\
\hline$\frac{1}{8}$ wild. . & 8 & 0 & 0 & 100 \\
\hline$\frac{1}{16}$ wild..... & 29 & 0 & 0 & 100 \\
\hline$\frac{1}{32}$ wild...... & 21 & 0 & 0 & 100 \\
\hline DARK. & & & & \\
\hline $\begin{array}{l}\text { 1 wild... } \\
\text { LoHT. }\end{array}$ & 0 & 1 & 1 & 0 \\
\hline$\frac{1}{4}$ wild . . . . & 7 & 0 & 17 & 41 \\
\hline$\frac{7}{8}$ wild... & 5 & 0 & 0 & 100 \\
\hline
\end{tabular}

Table 14.

Matings of wild hybrid females, heterozygous in agouti and with dark ticked belly, with guinea-pig males, also heterozygous in agouti but with light belly.

\begin{tabular}{|c|c|c|c|c|c|}
\hline \multicolumn{3}{|c|}{ Parents. } & \multicolumn{3}{|c|}{ Offspring. } \\
\hline Color. & Dark. & Light. & Iight. & Dark. & Non-agouti. \\
\hline Formula. & 우연 & $\sigma^{\top} \mathrm{Aa}$ & $\mathbf{A}^{\prime} \mathbf{A}$ or $\mathbf{A a}$ & $\mathbf{A}^{\prime} \mathbf{a}$ & aa \\
\hline & $\begin{array}{l}108 \\
108 \\
131 \\
166 \\
172 \\
198 \\
203 \\
219 \\
536\end{array}$ & $\begin{array}{l}1436 \\
1917 \\
2196 \\
2196 \\
1917 \\
2002 \\
-98 \\
-98 \\
1917\end{array}$ & $\begin{array}{l}1 \\
4 \\
1 \\
4 \\
1 \\
3 \\
1 \\
4 \\
0\end{array}$ & $\begin{array}{l}3 \\
1 \\
0 \\
3 \\
2 \\
1 \\
0 \\
2 \\
1\end{array}$ & $\begin{array}{l}0 \\
0 \\
0 \\
0 \\
0 \\
0 \\
0 \\
3 \\
1\end{array}$ \\
\hline & \multicolumn{2}{|c|}{$\begin{array}{r}\text { Total. . ........ } \\
\text { Most probable expec- } \\
\text { tation.............. } \\
\text { Zygotic formula proven. }\end{array}$} & $\overbrace{9_{3}^{9 A^{\prime}+9 A a}}^{19}$ & $\overbrace{\substack{9 A^{\prime} a \\
4}}^{13}$ & $\overbrace{9 \mathrm{aa}}^{4}$ \\
\hline
\end{tabular}


TABLE 15.

Matings of $\frac{1}{10}$ wild females, carrying wild and tame agouti, with guineapig males lacking agouti.

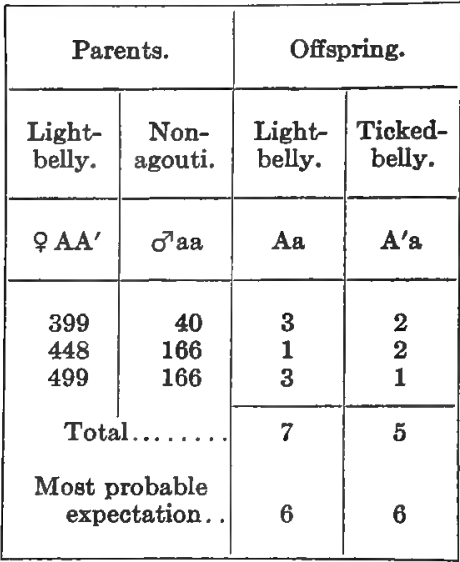

TABLE 16.

Matings of 1 wild females, in which all the offspring are black.

\begin{tabular}{|c|c|c|}
\hline \multicolumn{2}{|c|}{ Parents. } & Offspring. \\
\hline \multicolumn{2}{|c|}{$\begin{array}{l}\text { } \mathrm{BB} \times \sigma^{7} \mathrm{BB} \\
\text { 으 } \times \sigma^{7} \mathrm{BB}\end{array}$} & $\mathrm{BB}$ or $\mathrm{Bb}$ \\
\hline 87 & 170 & 2 \\
\hline 90 & 214 & 3 \\
\hline 91 & 214 & $\mathbf{3}$ \\
\hline 96 & 12612 & 12 \\
\hline 96 & 170 & 2 \\
\hline 97 & 11030 & 1 \\
\hline 98 & 12612 & 2 \\
\hline 101 & 12612 & 4 \\
\hline 107 & 199 & 1 \\
\hline 115 & 201 & 2 \\
\hline 122 & 12612 & 3 \\
\hline 124 & 11030 & 2 \\
\hline 150 & 201 & 1 \\
\hline 263 & 12612 & 2 \\
\hline 550 & 199 & 2 \\
\hline 605 & 170 & 5 \\
\hline 606 & 201 & 3 \\
\hline 642 & 199 & 2 \\
\hline 645 & 199 & 2 \\
\hline 797 & 4 & 1 \\
\hline 842 & 199 & 1 \\
\hline $\left.\begin{array}{r}98 \\
122\end{array}\right\}$ & 12612 & 6 \\
\hline $\left.\begin{array}{r}96 \\
122\end{array}\right\}$ & 12612 & 5 \\
\hline & & 67 \\
\hline
\end{tabular}

TABLE 17.

Matings of t wild females in which all the offspring are black.

\begin{tabular}{|c|c|c|}
\hline \multicolumn{2}{|c|}{ Parents. } & \multirow{2}{*}{$\begin{array}{c}\begin{array}{c}\text { Off- } \\
\text { spring. }\end{array} \\
\text { BB or Bb }\end{array}$} \\
\hline$\underset{q \mathrm{Bb} \text { or } \mathrm{bb} \times}{\mathrm{X}}$ & $\begin{array}{l}\sigma^{7} \mathrm{BB} \text { or } \mathrm{Bb} \\
\sigma^{2} \mathrm{BB}\end{array}$ & \\
\hline 127 & 1881 & 1 \\
\hline 127 & 2034 & 2 \\
\hline 135 & 1961 & 3 \\
\hline 140 & 1881 & 6 \\
\hline 166 & 85 & 2 \\
\hline 197 & 119 & 2 \\
\hline 222 & 1881 & 2 \\
\hline 222 & 2034 & 5 \\
\hline 252 & 85 & 2 \\
\hline 264 & 85 & 3 \\
\hline 296 & 117 & 1 \\
\hline 311 & 1961 & 2 \\
\hline 311 & 2278 & 2 \\
\hline 312 & 1961 & 2 \\
\hline 312 & 98 & 1 \\
\hline 471 & 96 & 5 \\
\hline 475 & 96 & 1 \\
\hline 574 & $\mathrm{G}$ & 2 \\
\hline 576 & 99 & 4 \\
\hline 577 & 15 & 2 \\
\hline 599 & 2278 & 5 \\
\hline 659 & 117 & 1 \\
\hline 671 & 96 & 3 \\
\hline 723 & 117 & 1 \\
\hline 793 & 96 & $I$ \\
\hline 837 & 98 & 1 \\
\hline $\left.\begin{array}{l}135 \\
153\end{array}\right\}$ & 1961 & 2 \\
\hline $\left.\begin{array}{l}135 \\
310 \\
312\end{array}\right\}$ & 1961 & 5 \\
\hline $\left.\begin{array}{l}574 \\
577\end{array}\right\}$ & 99 & 4 \\
\hline $\left.\begin{array}{l}815 \\
816\end{array}\right\}$ & 99 & 3 \\
\hline Total... & $\ldots \ldots \ldots \ldots$ & 76 \\
\hline
\end{tabular}


TABLe 18.

Tabue 19.

Matings of $\frac{1}{16}$ wild females, in which all the offspring are black. Matings of $\frac{1}{32}$ wild females, in

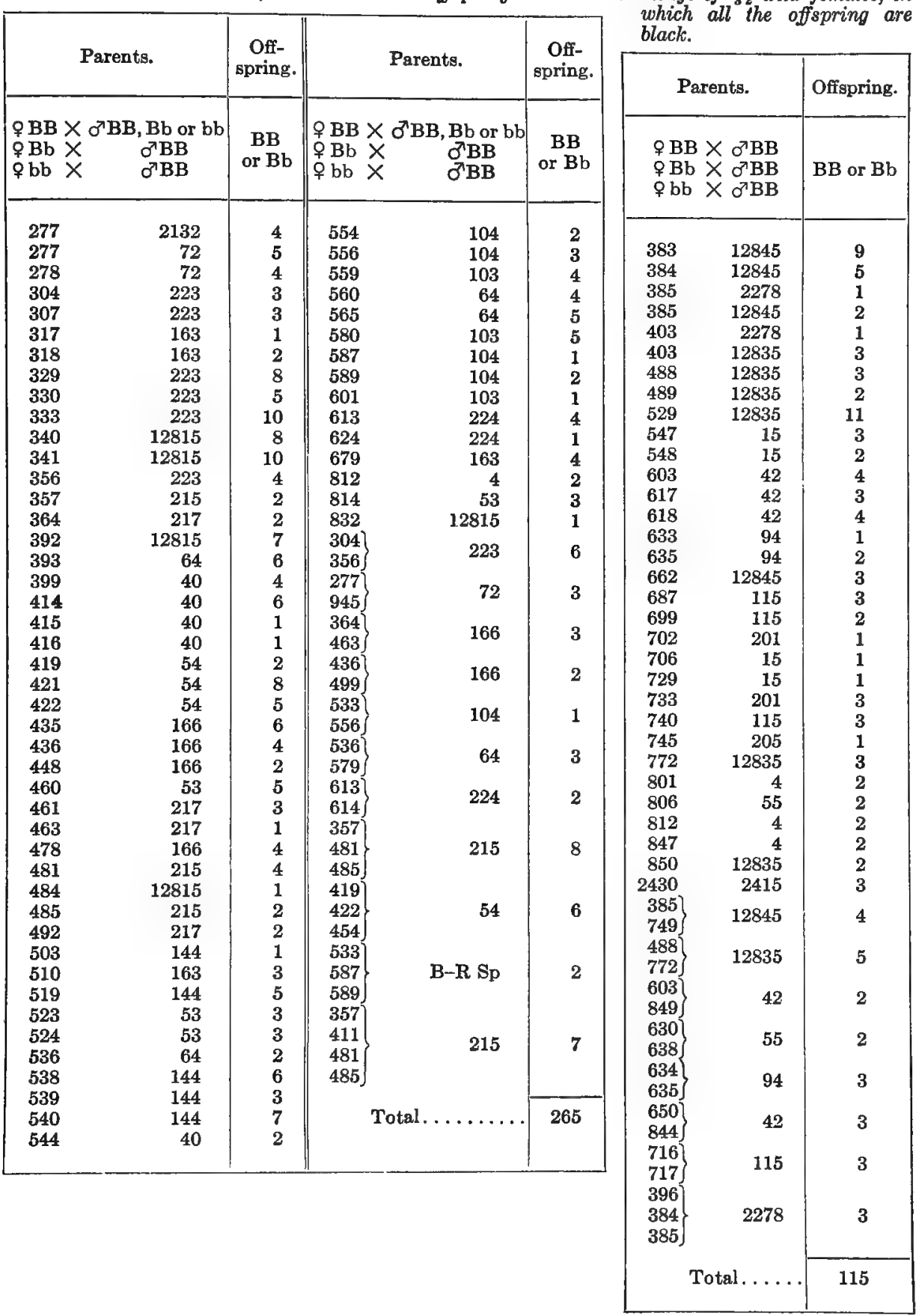


Table 20.

Matings of 1 wild females, in which all the offspring are black.

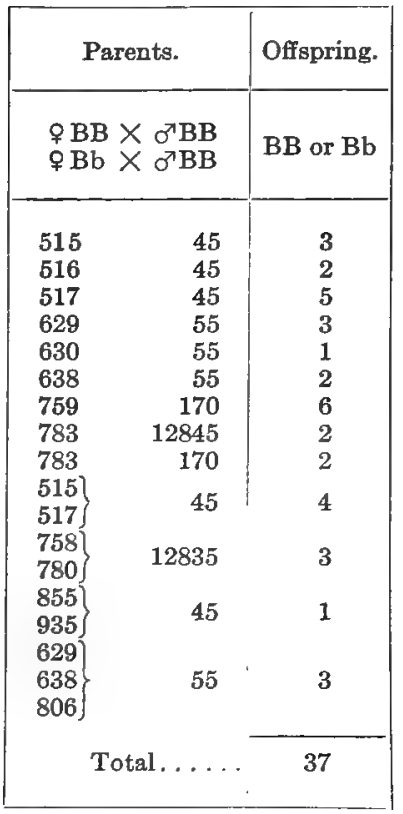

TABle 23.

Matings of $\frac{1}{8}$ wild females heterozygous in black, with brown guinea-pig males.

\begin{tabular}{|c|c|c|c|}
\hline \multicolumn{2}{|c|}{ Parents. } & \multicolumn{2}{|c|}{ Offspring. } \\
\hline \multicolumn{2}{|c|}{ 오 $\mathrm{Bb} \times \sigma^{7} \mathrm{bb}$} & \multicolumn{2}{|c|}{$\mathrm{Bb}+\mathrm{bb}$} \\
\hline 127 & 1541 & 0 & 1 \\
\hline 131 & 9758 & 0 & 1 \\
\hline 131 & 2196 & 1 & 1 \\
\hline 145 & 2196 & 6 & 4 \\
\hline 166 & 2196 & 6 & 0 \\
\hline 177 & 1923 & 3 & 0 \\
\hline 178 & 1923 & 10 & 3 \\
\hline 207 & 2083 & 1 & 1 \\
\hline 208 & 2083 & 2 & 2 \\
\hline 209 & 2083 & 0 & 2 \\
\hline 215 & -30 & 0 & 1 \\
\hline 215 & -28 & 4 & 0 \\
\hline 219 & -98 & 4 & 5 \\
\hline 240 & 2366 & 2 & 2 \\
\hline 242 & 2366 & 0 & 3 \\
\hline 367 & 2196 & 1 & 2 \\
\hline 402 & 2366 & 2 & 1 \\
\hline 470 & 2036 & 1 & 2 \\
\hline \multirow{2}{*}{\multicolumn{2}{|c|}{$\begin{array}{r}\text { Total.... } \\
\text { Expected.... }\end{array}$}} & 43 & 31 \\
\hline & & 37 & 37 \\
\hline
\end{tabular}

Table 21.

Summary of Tables 2,6 , and 16-20 (all offspring are black pigmented).

\begin{tabular}{|c|c|c|c|}
\hline Table. & Females. & Males. & $\mathrm{BB}$ or $\mathrm{Bb}$ \\
\hline 2 & Guinea-pig. . & Pure wild.. & 37 \\
\hline 6 & $\frac{1}{2}$ wild & Guinea-pig. & 83 \\
\hline 16 & $\frac{1}{4}$ wild. . & Guinea-pig. & 67 \\
\hline 17 & $\frac{1}{8}$ wild. & Guinea-pig. & 76 \\
\hline 18 & ${ }_{16}^{1}$ wild... & Guinea-pig. & 265 \\
\hline 19 & Je wild. . & Guinea-pig. & 115 \\
\hline 20 & $\frac{1}{64}$ wild. . & Guinea-pig. & 37 \\
\hline \multicolumn{2}{|c|}{ Total. } & & 680 \\
\hline
\end{tabular}

TABLE 22.

Matings of $\frac{1}{4}$ wild females, heterozygous in black, with brown guinea-pig males.

\begin{tabular}{|c|c|c|c|}
\hline \multicolumn{2}{|c|}{ Parents. } & \multicolumn{2}{|c|}{ Offispring. } \\
\hline \multicolumn{2}{|c|}{$\% \mathrm{Bb} \times \sigma^{\top} \mathrm{bb}$} & \multicolumn{2}{|c|}{$\mathrm{Bb}+\mathrm{bb}$} \\
\hline 89 & 9758 & 0 & 2 \\
\hline 90 & 1541 & 5 & 4 \\
\hline 91 & 1541 & 4 & 5 \\
\hline 92 & 9246 & 0 & 1 \\
\hline 92 & 1541 & 0 & 2 \\
\hline 95 & 1541 & 1 & 0 \\
\hline 105 & 1541 & 6 & 3 \\
\hline 106 & 1541 & 1 & 0 \\
\hline 106 & 0 & 1 & 0 \\
\hline 107 & 9758 & 2 & 1 \\
\hline 107 & 617 & 1 & 1 \\
\hline 107 & 25 & 2 & 1 \\
\hline 110 & 9578 & 1 & 3 \\
\hline 110 & 617 & 4 & 1 \\
\hline 113 & 1543 & 3 & 3 \\
\hline 115 & 1543 & 6 & 7 \\
\hline 119 & 1543 & 9 & 5 \\
\hline $\begin{array}{c}147 \\
90\end{array}$ & 1543 & 2 & 0 \\
\hline 95$\}$ & 1541 & 3 & 1 \\
\hline 105) & & & \\
\hline $\left.\begin{array}{r}91 \\
106\end{array}\right\}$ & 1541 & 3 & 1 \\
\hline $\left.\begin{array}{l}113 \\
119\end{array}\right\}$ & 1543 & 3 & 0 \\
\hline $\left.\begin{array}{r}92 \\
148\end{array}\right\}$ & 1541 & 0 & 4 \\
\hline & al..... & 57 & 45 \\
\hline $\begin{array}{r}\text { Most } \\
\text { exp }\end{array}$ & $\begin{array}{l}\text { obable } \\
\text { ation. }\end{array}$ & & 51 \\
\hline
\end{tabular}


TaBle 24.

Matings of th and $\frac{1}{82}$ wild females with guinea-pig males, in which one parent is heterozygous in black and the other is brown.

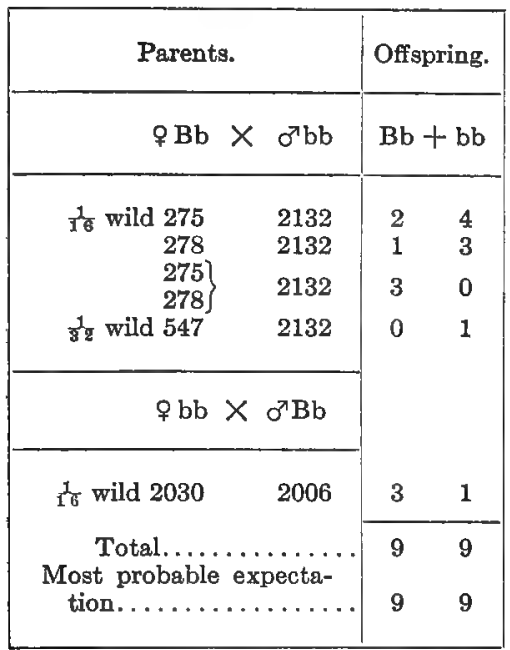

TABLE 25.

Summary of Tables 20-24, in which we expect an equality of black and brown offspring.

\begin{tabular}{|c|c|c|c|c|}
\hline \multirow{2}{*}{ Table. } & \multicolumn{2}{|c|}{ Parents. } & \multirow{2}{*}{\multicolumn{2}{|c|}{ Offspring. }} \\
\hline & Females. & Males. & & \\
\hline $\begin{array}{l}22 \\
23\end{array}$ & $\begin{array}{l}\frac{1}{2} \text { wild...... } \\
\frac{2}{8} \text { wild ..... }\end{array}$ & $\begin{array}{l}\text { Guinea-pig. . . } \\
\text { Guinea-pig. . }\end{array}$ & $\begin{array}{l}57 \\
43\end{array}$ & $\begin{array}{l}45 \\
31\end{array}$ \\
\hline 24 & $\left\{\begin{array}{l}16 \text { wild.. } \\
\frac{1}{32} \text { wild. }\end{array}\right.$ & Guinea-pig. . . & 9 & 9 \\
\hline Most & $\begin{array}{l}\text { Total......... } \\
\text { orobable expe }\end{array}$ & & $\begin{array}{r}109 \\
97\end{array}$ & $\begin{array}{l}85 \\
97\end{array}$ \\
\hline
\end{tabular}

TABLE 26.

Matings of $\frac{1}{8}$ wild females with a guinea-pig male, all heterozygous in black.

\begin{tabular}{|rr|cr|}
\hline \multicolumn{2}{|c|}{ Parents. } & Offspring. \\
\hline \multicolumn{2}{|c|}{$9 \mathrm{Bb} \times \sigma^{7} \mathrm{Bb}$} & $\mathrm{BB}$ or $\mathrm{Bb}+\mathrm{bb}$ \\
\hline 208 & 2002 & 2 & 1 \\
264 & 2002 & 5 & 0 \\
\cline { 2 - 3 } $\begin{array}{r}\text { Total..... } \\
\text { Most probable } \\
\text { expectation. }\end{array}$ & 7 & 1 \\
\hline
\end{tabular}

TABLE 28.

Matings of $\frac{1}{16}$ and $\frac{1}{32}$ wild brown females with brown guinea-pig males.

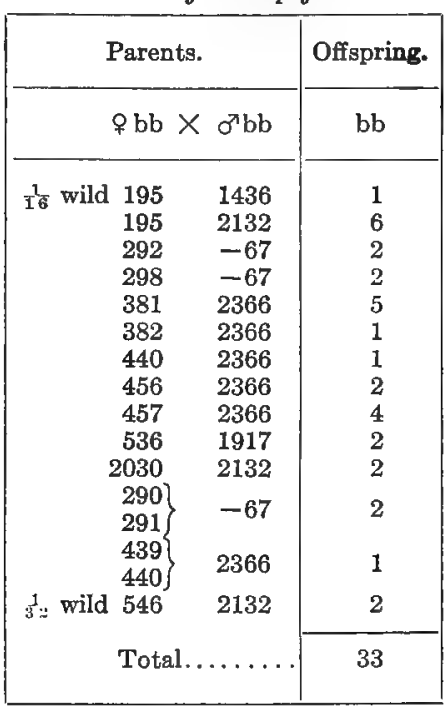

TABLE 27.

Matings of $\frac{1}{8}$ wild brown females with brown guineapig males.

\begin{tabular}{|c|c|c|}
\hline \multicolumn{2}{|c|}{ Parents. } & Offspring. \\
\cline { 1 - 2 } ㅇ bb $\times$ & o'bb & $\mathrm{bb}$ \\
\hline 108 & 1436 & 4 \\
108 & 1917 & 5 \\
130 & 2036 & 6 \\
130 & 1541 & 2 \\
170 & -30 & 4 \\
170 & -28 & 2 \\
172 & 1917 & 3 \\
173 & 1917 & 7 \\
180 & 1917 & 3 \\
197 & -98 & 7 \\
203 & -98 & 1 \\
205 & -98 & 4 \\
232 & 1543 & 1 \\
232 & 2366 & 2 \\
237 & 2036 & 4 \\
238 & 2366 & 3 \\
248 & 2157 & 3 \\
$137\}$ & 2157 & 3 \\
$248\}$ & & \\
197 & 1541 & 3 \\
$203\}$ & & \\
197 & -98 & 6 \\
203 & & \\
$172\}$ & 1917 & 5 \\
$180\}$ & & \\
& Total. & 78 \\
& & \\
\hline & & \\
\hline
\end{tabular}


Table 29.

Summary of Tables 27 and 28; matings of brown female hybrids and brown guinea-pig males.

\begin{tabular}{|c|c|c|c|}
\hline \multirow{2}{*}{ Table. } & \multicolumn{2}{|c|}{ Parents. } & \multirow{2}{*}{$\frac{\text { Off spring. }}{\mathrm{bb}}$} \\
\hline & Females bb. & Males bb. & \\
\hline 27 & $\frac{1}{8}$ wild. . & Guinea-pig. . & 78 \\
\hline 28 & $\frac{1}{16}$ wild. & Guinea-pig. . & 31 \\
\hline 28 & $\frac{1}{32}$ wild. . & Guinea-pig. . & 2 \\
\hline \multicolumn{3}{|c|}{ 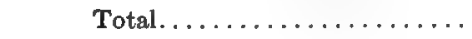 } & 111 \\
\hline
\end{tabular}

TABle 31.

Matings of $\frac{1}{2}$ wild females, homozygous in extension, with guinea-pig males carrying restriction.

\begin{tabular}{|c|c|c|}
\hline \multicolumn{2}{|c|}{ Parents. } & Offspring. \\
\hline \multicolumn{2}{|c|}{ क EE $\times \sigma^{\top} \mathrm{Ee}$ or ee } & $\mathrm{EE}$ or $\mathrm{Ee}$ \\
\hline 68 & 4 & 5 \\
\hline 69 & 4 & 2 \\
\hline 72 & 617 & 4 \\
\hline 75 & 9246 & 2 \\
\hline 253 & 617 & 1 \\
\hline $\left.\begin{array}{c}75 \\
\frac{1}{2} \text { wild }\end{array}\right\}$ & 9246 & 3 \\
\hline$\frac{1}{2}$ wild & 9246 & 12 \\
\hline & $\ldots \ldots \ldots$ & 29 \\
\hline
\end{tabular}

Table 32.

Malings of 1 wild females with guinea-pig males, in which one parent only carries restriction.

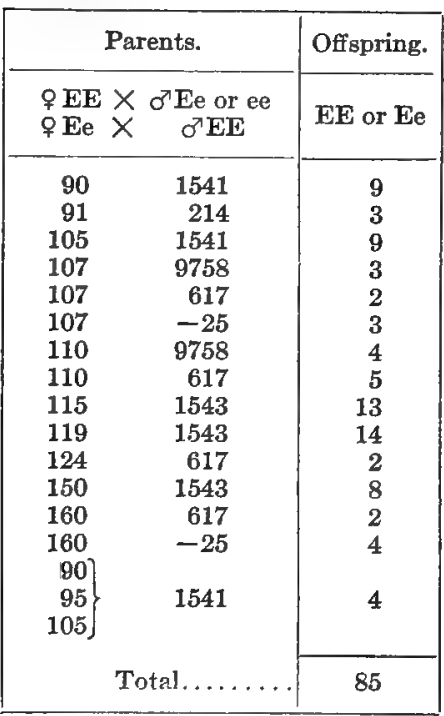

Table 30.

Matings of guinea-pig females, heterozygous in extension, with a wild Cavia rufescens male.

\begin{tabular}{|c|c|c|}
\hline \multicolumn{2}{|c|}{ Parents. } & Offspring. \\
\hline \multicolumn{2}{|c|}{ 우 $\mathrm{Ee} \times \sigma^{\top} \mathrm{EE}$} & EE or Ee \\
\hline $\begin{array}{l}9470 \\
9473\end{array}$ & $\begin{array}{l}33 \\
33\end{array}$ & $\begin{array}{l}3 \\
3 \\
\end{array}$ \\
\hline \multicolumn{2}{|c|}{ Total.... } & 6 \\
\hline
\end{tabular}

TABLe 33.

Matings of 1 wild females with guinea-pio males, in which one parent only carries restriction.

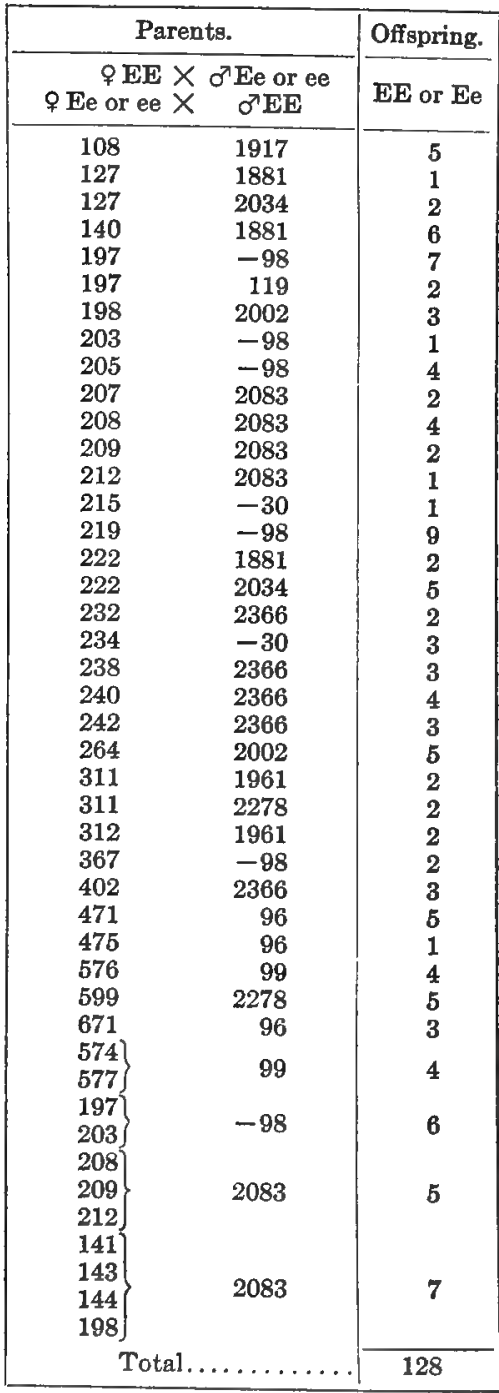


Table 34.

Matings of $\frac{1}{16}$ wild females with guinea-pig males, in which one parent only carries restriction.

\begin{tabular}{|c|c|c|c|c|c|}
\hline \multicolumn{2}{|c|}{ Parents. } & Offspring. & \multicolumn{2}{|c|}{ Parents. } & Offspring. \\
\hline \multicolumn{2}{|c|}{$\begin{aligned} & \text { क EE } \times \times \sigma^{\top} \mathrm{Ee} \text { or ee } \\
& \text { \& Ee or ee } \times \quad \sigma^{2} \mathrm{EE}\end{aligned}$} & $\mathrm{EE}$ or $\mathrm{Ee}$ & \multicolumn{2}{|c|}{$\begin{array}{c}\text { ㅇ EE } \times \sigma^{\lambda} \mathrm{Ee} \text { or ee } \\
\text { ㅇ Ee or ee } \times \\
\delta^{\prime} \mathrm{EE}\end{array}$} & EE or ee \\
\hline 195 & 1436 & 1 & 524 & 53 & 3 \\
\hline 195 & 2132 & 6 & 536 & 64 & 2 \\
\hline 275 & 2132 & 6 & 538 & 144 & 6 \\
\hline 277 & 2132 & 4 & 539 & 144 & 3 \\
\hline 277 & 72 & 5 & 540 & 144 & 7 \\
\hline 278 & 2132 & 3 & 544 & 40 & 1 \\
\hline 278 & 72 & 4 & 554 & 104 & 2 \\
\hline 304 & 223 & 3 & 556 & 104 & 3 \\
\hline 307 & 223 & 3 & 559 & 103 & 4 \\
\hline 329 & 223 & 8 & 560 & 64 & 4 \\
\hline 330 & 223 & 5 & 565 & 64 & $\mathbf{5}$ \\
\hline 341 & 12815 & 7 & 580 & 103 & 5 \\
\hline 356 & 223 & 4 & 587 & 104 & 1 \\
\hline 357 & 215 & 2 & 589 & 104 & 2 \\
\hline 364 & 217 & 2 & 601 & 103 & 1 \\
\hline 381 & 2366 & 5 & 613 & 224 & 4 \\
\hline 382 & 2366 & 1 & 614 & 224 & 2 \\
\hline 393 & 64 & 6 & 621 & 224 & 1 \\
\hline 399 & 40 & 4 & 679 & 163 & 4 \\
\hline 414 & 40 & 6 & 814 & 53 & 3 \\
\hline 415 & 40 & 1 & 304\} & 223 & 6 \\
\hline 416 & 40 & 1 & $356\}$ & & \\
\hline 419 & 54 & 2 & $277\}$ & & 3 \\
\hline 421 & 54 & 8 & $945\}$ & 72 & 3 \\
\hline 422 & 54 & 5 & $364\}$ & & 3 \\
\hline 435 & 166 & 4 & $463\}$ & 217 & 3 \\
\hline 436 & 166 & 3 & $436\}$ & 166 & 2 \\
\hline 440 & 2366 & 1 & $499\}$ & 106 & 2 \\
\hline 448 & 166 & 2 & $613\}$ & 224 & 2 \\
\hline 453 & 54 & 2 & $614\}$ & & \\
\hline 456 & 2366 & 2 & $357\}$ & & \\
\hline 457 & 2366 & 4 & $481\}$ & 215 & 8 \\
\hline 460 & 53 & 5 & 485 & & \\
\hline 461 & 217 & 3 & 419 & & \\
\hline 463 & 217 & 1 & $422\}$ & 54 & 6 \\
\hline 478 & 176 & 4 & 454] & & \\
\hline 481 & 215 & 4 & $357 ?$ & & \\
\hline 485 & 215 & 2 & 411 & 215 & 7 \\
\hline 492 & 217 & 2 & 481 & 210 & 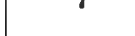 \\
\hline 503 & 144 & 1 & $485]$ & & \\
\hline 519 & 144 & 5 & & & \\
\hline 523 & 53 & 3 & To & & 250 \\
\hline
\end{tabular}


TABLE 35.

Matings of $\frac{1}{32}$ wild females with guineapig males, in which one parent only (or neither) carries restriction.

\begin{tabular}{|c|c|c|}
\hline \multicolumn{2}{|c|}{ Parents. } & Offspring. \\
\hline \multicolumn{2}{|c|}{ 으 $\mathrm{EE} \times \sigma^{\top} \mathrm{EE}$ or $\mathrm{Ee}$} & $\mathbf{E E}$ or $\mathbf{E e}$ \\
\hline 383 & 12845 & 9 \\
\hline 384 & 12845 & 5 \\
\hline 385 & 2278 & 1 \\
\hline 385 & 12845 & 2 \\
\hline 403 & 2278 & 1 \\
\hline 403 & 12835 & 3 \\
\hline 488 & 12835 & 3 \\
\hline 489 & 12835 & 2 \\
\hline 529 & 12835 & 11 \\
\hline 546 & 2132 & 2 \\
\hline 547 & 2132 & 1 \\
\hline 547 & 15 & 3 \\
\hline 548 & 15 & 2 \\
\hline 603 & 42 & 4 \\
\hline 617 & 42 & 3 \\
\hline 618 & 42 & 4 \\
\hline 633 & 94 & 1 \\
\hline 635 & 94 & 2 \\
\hline 662 & 12845 & 3 \\
\hline 687 & 115 & 3 \\
\hline 702 & 201 & 1 \\
\hline 733 & 201 & 3 \\
\hline 745 & 201 & 1 \\
\hline 806 & 55 & 2 \\
\hline 812 & 4 & 2 \\
\hline 847 & 4 & 2 \\
\hline 2430 & 2415 & 3 \\
\hline $\left.\begin{array}{l}385 \\
749\end{array}\right\}$ & 12845 & 4 \\
\hline $\left.\begin{array}{l}603 \\
849\end{array}\right\}$ & 42 & 2 \\
\hline $\left.\begin{array}{l}630 \\
638\end{array}\right\}$ & 55 & 2 \\
\hline $\left.\begin{array}{l}634 \\
635\end{array}\right\}$ & 94 & 3 \\
\hline 650 & 42 & 3 \\
\hline 844$\}$ & & \\
\hline $\left.\begin{array}{l}384 \\
385\end{array}\right\}$ & 2278 & 3 \\
\hline & & 96 \\
\hline
\end{tabular}

TABLE 36.

Matings of $\frac{1}{6}$ wild females with guinea-pig males, in which one parent only (or neither) carries restriction.

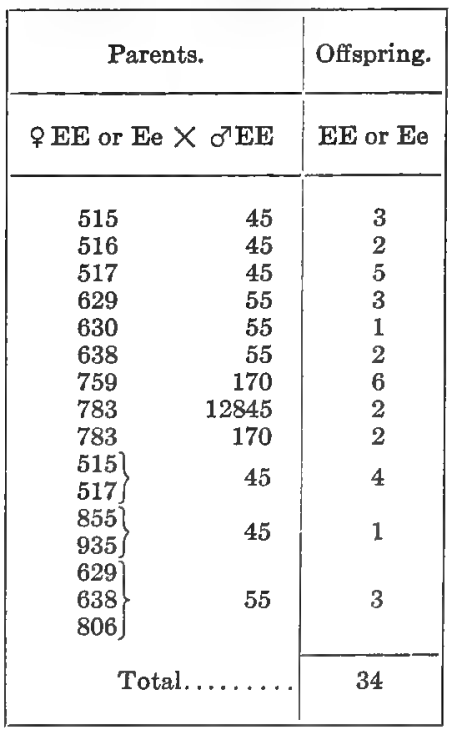

TABLE 37.

Summary of Tables 30-36, in which animals of extended pigmentation are expected, since only one parent (or neither) carries restriction.

\begin{tabular}{|c|c|c|c|}
\hline \multirow{2}{*}{ Table. } & \multicolumn{2}{|c|}{ Parents. } & \multirow{2}{*}{$\begin{array}{l}\text { Offspring. } \\
\mathrm{EE} \text { or } \mathrm{Ee}\end{array}$} \\
\hline & Females. & Males. & \\
\hline 30 & Guinea-pig. . & Wild..... . . & 6 \\
\hline 31 & $\frac{1}{2}$ wild. ... & Guinea-pig. ... & 29 \\
\hline 32 & thild. . & Guinea-pig. . . . & 85 \\
\hline 33 & $\frac{1}{8}$ wild.... & Guinea-pig.... & 128 \\
\hline 34 & 18 wild... & Guinea-pig.... & 250 \\
\hline 35 & $\frac{1}{32}$ wild. . & Guinea-pig.... . & 96 \\
\hline \multirow[t]{2}{*}{36} & $\frac{1}{64}$ wild. . & Guinea-pig.... & 34 \\
\hline & Total. & & 628 \\
\hline
\end{tabular}


Table 38.

Matings of $\frac{1}{2}$ wild females, heterozygous in the extension factor, with guinea-pig males lacking it.

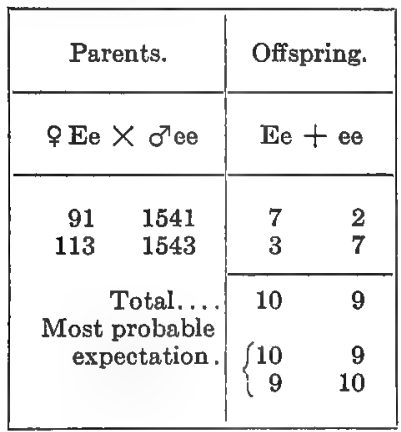

TABLE 40.

Matings of $\frac{1}{16}$ wild females with guinea-pig males, in which one parent is heterozygous in the extension factor and the other parent lacks it.

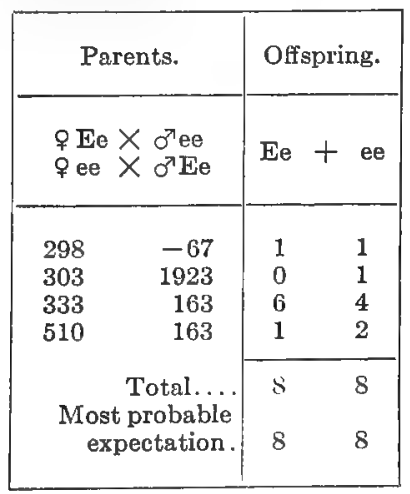

\section{TABLE 42.}

Summary of Tables $38-41$, in which we expect an equality of animals of extended pigmentation and restricted pigmentation.

\begin{tabular}{|c|c|c|c|c|}
\hline \multirow{2}{*}{ Table. } & \multicolumn{2}{|c|}{ Parents. } & \multicolumn{2}{|c|}{ Offspring. } \\
\hline & Females. & Males. & $\mathrm{Ee}$ & ee \\
\hline 38 & $\frac{1}{4}$ wild. . & Guinea-pig.... & 10 & 9 \\
\hline 39 & 音 wild. . & Guinea-pig.... . & 22 & 32 \\
\hline 40 & $\frac{1}{16}$ wild. . & Guinea-pig.... & 8 & 8 \\
\hline 41 & उ't wild. . & Guinea-pig.... & 5 & 5 \\
\hline 41 & $\frac{1}{61}$ wild.. & Guinea-pig. ... & 2 & 1 \\
\hline \multicolumn{3}{|c|}{ Total................... } & 47 & 55 \\
\hline \multicolumn{3}{|c|}{ Most probable expectation......... } & 51 & 51 \\
\hline
\end{tabular}

TABLE 39.

Matings of wild females with guinea-pig males, in which one parent is heterozygous in the extension factor and the other parent lacks it.

\begin{tabular}{|c|c|c|c|}
\hline \multicolumn{2}{|c|}{ Pareats. } & \multicolumn{2}{|c|}{ Offspring } \\
\hline $\begin{array}{l}\text { \% } \mathrm{Ee} \\
\text { † ee }\end{array}$ & $\begin{array}{l}\sigma^{\top} \mathrm{ee} \\
\sigma^{7} \mathrm{Ee}\end{array}$ & \multicolumn{2}{|c|}{$\mathrm{Ee}+\mathrm{ee}$} \\
\hline 127 & 1541 & 0 & 1 \\
\hline 130 & 2036 & 2 & 4 \\
\hline 130 & 1541 & 1 & 1 \\
\hline 131 & 9758 & 0 & 1 \\
\hline 131 & 2196 & 1 & 1 \\
\hline 145 & 2196 & 2 & 8 \\
\hline 166 & 2196 & 5 & 1 \\
\hline 170 & -38 & 0 & 2 \\
\hline 172 & 1917 & 2 & 1 \\
\hline 180 & 1917 & 0 & 3 \\
\hline 237 & 2936 & 2 & 2 \\
\hline 367 & 2196 & 2 & 1 \\
\hline 470 & 2036 & 2 & 1 \\
\hline $\begin{array}{l}197 \\
203\end{array}$ & 1541 & 2 & 1 \\
\hline $\begin{array}{l}172 \\
180\end{array}$ & 1917 & 1 & 4 \\
\hline & otal.... & 22 & 32 \\
\hline $\begin{array}{r}\text { Mo } \\
\text { e }\end{array}$ & $\begin{array}{l}\text { obable } \\
\text { tation. }\end{array}$ & 27 & 27 \\
\hline
\end{tabular}

\section{TABLE 41.}

Matings of $\frac{1}{32}$ wild and 1 wild females with guinea-pig males, in which one parent is heterozygous in the extension factor and the other parent lacks it.

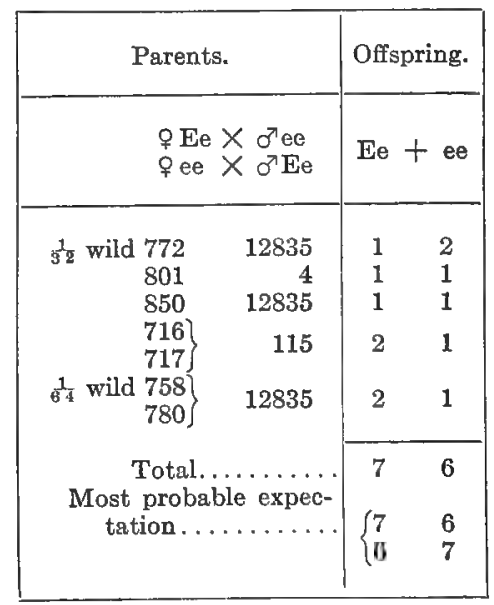


TABLE 43.

Matings of wild female hybrids with guineapig males, in which both parents were heterozygous in the extension factor.

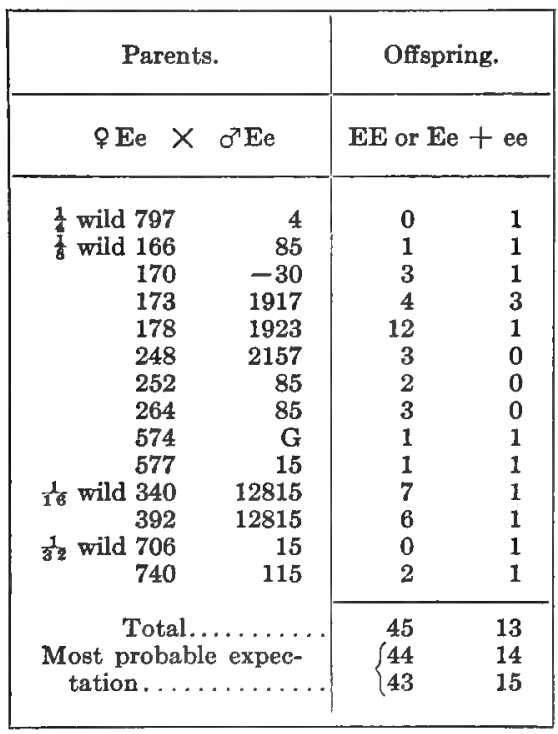

TABLE 44.

Matings of albino guinea-pig females with wild Cavia rufescens males.

\begin{tabular}{|c|c|c|}
\hline \multicolumn{2}{|c|}{ Parents. } & Offspring. \\
\hline \multicolumn{2}{|c|}{ q ce $\times \sigma^{\top} \mathrm{CC}$} & $\mathrm{Ce}$ \\
\hline 1125 & 1 & 11 \\
\hline 1625 & 1 & 4 \\
\hline 3024 & 1 & 1 \\
\hline 9536 & 33 & 2 \\
\hline \multicolumn{2}{|c|}{ Total... } & 18 \\
\hline
\end{tabular}

TABLE 45.

Matings of $\frac{1}{2}$ wild females with guinea-pig males carrying albinism as a recessive character.

\begin{tabular}{|c|c|c|}
\hline \multicolumn{2}{|c|}{ Parents. } & Offspring. \\
\hline \multicolumn{2}{|c|}{ q $\mathrm{CC} \times \sigma^{\top} \mathrm{Ce}$} & $\mathrm{CC}$ or $\mathrm{Ce}$ \\
\hline 63 & 2193 & 4 \\
\hline 68 & 2193 & 2 \\
\hline $\begin{array}{l}75 \\
69\}\end{array}$ & 9246 & 2 \\
\hline $\left.\begin{array}{r}118 \\
75\end{array}\right\}$ & 2193 & 4 \\
\hline$\frac{1}{2}$ wild & 9246 & 3 \\
\hline$\frac{1}{2}$ wild & 9246 & 12 \\
\hline \multicolumn{2}{|c|}{ Total...... } & 27 \\
\hline
\end{tabular}

TABLE 46.

Matings of wild hybrid females and guinea-pig males, in which one parent only carries albinism.

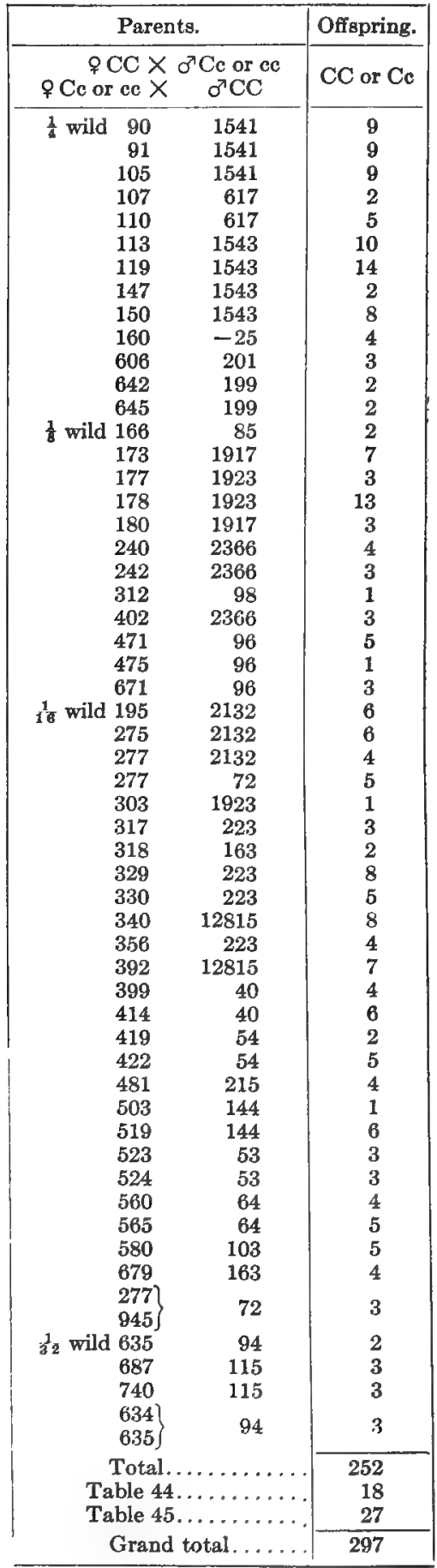


TABLES.

TABLE 47.

Matings of 1 wild females, heterozygous in color, with albino guinea-pig males.

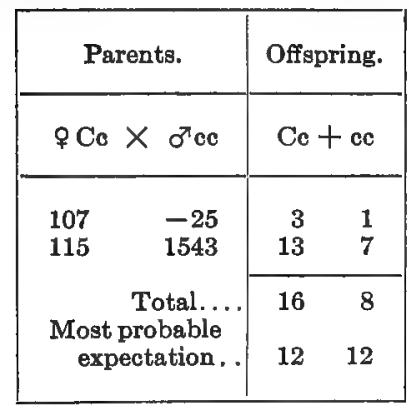

TABLE 49.

Matings of $\frac{1}{16}$ and $\frac{1}{32}$ wild females with guinea-pig males, in which one parent is heterozygous in color and the other is an albino.

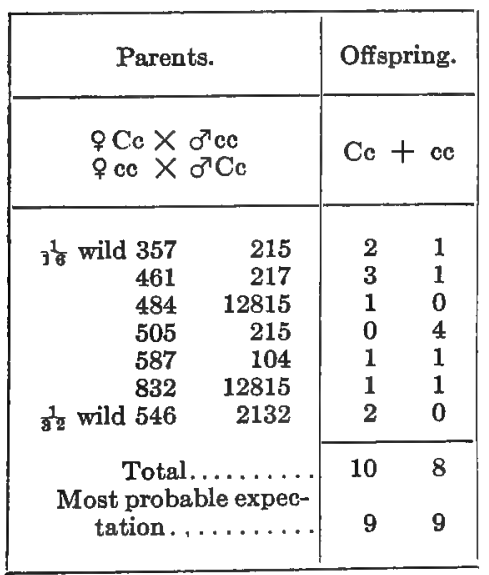

TABLE 51.

Matings of $\frac{1}{2}$ wild females with guineapig males, in which both parents are heterozygous in color.

\begin{tabular}{|c|c|c|c|}
\hline \multicolumn{2}{|c|}{ Parents. } & \multicolumn{2}{|c|}{ Offspring. } \\
\hline 90 & $\sigma^{2} \mathrm{Cc}$ & $\mathrm{CC}$ or & $-c o$ \\
\hline 107 & $\begin{array}{l}9758 \\
9758 \\
1170\end{array}$ & $\begin{array}{l}2 \\
3 \\
5\end{array}$ & $\begin{array}{l}1 \\
1 \\
1\end{array}$ \\
\hline & $\begin{array}{l}\text { al...... } \\
\text { robable } \\
\text { ctation. }\end{array}$ & $\left\{\begin{array}{r}10 \\
9 \\
10\end{array}\right.$ & $\begin{array}{l}3 \\
4 \\
3\end{array}$ \\
\hline
\end{tabular}

TABLE 48.

Matings of $\frac{1}{8}$ wild females with guinea-pig males, in which one parent is heterozygous in color, and the other is an albino.

\begin{tabular}{|c|c|c|c|}
\hline \multicolumn{2}{|c|}{ Parents. } & \multicolumn{2}{|c|}{ Offspring. } \\
\hline \multicolumn{2}{|c|}{$\begin{array}{l}q \mathrm{Cc} \times \sigma^{7} \mathrm{cc} \\
q \mathrm{cc} \times \mathrm{o}^{7} \mathrm{Cc}\end{array}$} & \multicolumn{2}{|c|}{$\mathrm{Ce}+\mathrm{ec}$} \\
\hline 143 & 2002 & I & 1 \\
\hline 144 & 2002 & 1 & 3 \\
\hline 166 & 2196 & 6 & 3 \\
\hline 207 & 2083 & 2 & 3 \\
\hline 208 & 2083 & 4 & 5 \\
\hline 209 & 2083 & 2 & 1 \\
\hline 212 & 2083 & 1 & 4 \\
\hline 232 & 1541 & 1 & 0 \\
\hline 312 & 1961 & 2 & 1 \\
\hline & 2083 & 5 & 6 \\
\hline $\left.\begin{array}{l}209 \\
212\end{array}\right\}$ & & & \\
\hline \multirow{2}{*}{\multicolumn{2}{|c|}{$\begin{array}{r}\text { Total..... } \\
\text { Most probable }\end{array}$}} & 25 & 27 \\
\hline & & 26 & 26 \\
\hline
\end{tabular}

TABLE 50.

Summary of Tables 47-49, in which we expect an equality of colored and albino offspring.

\begin{tabular}{|c|c|c|c|c|}
\hline \multirow{2}{*}{ Table. } & \multicolumn{2}{|c|}{ Parents. } & \multirow{2}{*}{\multicolumn{2}{|c|}{$\begin{array}{l}\text { Offspring. } \\
\mathrm{Ce}+\mathrm{cc}\end{array}$}} \\
\hline & Females. & Males. & & \\
\hline 47 & wild. & Guinea-pig . & 16 & 8 \\
\hline 48 & $\frac{1}{8}$ wild. & Guinea-pig. & 25 & 27 \\
\hline 49 & $\frac{1}{16}$ wild. & Guinea-pig. & 8 & 8 \\
\hline 49 & $\frac{1}{8 g}$ wild. & Guinea-pig. & 2 & 0 \\
\hline \multirow{2}{*}{\multicolumn{3}{|c|}{ 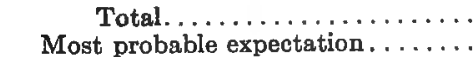 }} & 51 & 43 \\
\hline & & & 47 & 47 \\
\hline
\end{tabular}

TABLE 52.

Matings of $\frac{1}{8}$ wild females with guinea-pig males, in which both parents are heterozygous in color.

\begin{tabular}{|c|c|c|c|}
\hline \multicolumn{2}{|c|}{ Parents. } & \multicolumn{2}{|c|}{ Offspring. } \\
\hline \multicolumn{2}{|c|}{ $\$ \mathrm{Cc} \times \sigma^{7} \mathrm{Cc}$} & \multicolumn{2}{|c|}{$\mathrm{CC}$ or $\mathrm{Cc}+\mathrm{cc}$} \\
\hline 141 & 2002 & 1 & 0 \\
\hline 145 & 2196 & 8 & 3 \\
\hline 198 & 2002 & 3 & 1 \\
\hline 296 & 117 & 1 & 1 \\
\hline 361 & 2196 & 0 & 2 \\
\hline 574 & G & 2 & 1 \\
\hline 576 & 99 & 4 & 1 \\
\hline 577 & 15 & 2 & 1 \\
\hline $\left.\begin{array}{l}\mathbf{5 7 4} \\
\mathbf{5 7 7}\end{array}\right\}$ & 99 & 4 & 0 \\
\hline & Total.... & 25 & 10 \\
\hline \multirow{2}{*}{\multicolumn{2}{|c|}{$\begin{array}{l}\text { Most probable } \\
\text { expectation. }\end{array}$}} & $\{26$ & 9 \\
\hline & & $\{27$ & 8 \\
\hline
\end{tabular}


Tbale 53.

Mutings of 1 wild females with guineapig males, in which both parents are heterozygous in color.

\begin{tabular}{|c|c|c|c|}
\hline \multicolumn{2}{|c|}{ Parents. } & \multicolumn{2}{|c|}{ Offispring. } \\
\hline \multicolumn{2}{|c|}{ $9 \mathrm{Cc} \times \sigma^{\top} \mathrm{Cc}$} & \multicolumn{2}{|c|}{$\mathrm{CC}$ or $\mathrm{Cc}+\mathrm{cc}$} \\
\hline 278 & 2132 & 3 & 2 \\
\hline 278 & 72 & 4 & 1 \\
\hline 290 & -67 & 0 & 1 \\
\hline 292 & -67 & 2 & 1 \\
\hline 341 & 12815 & 7 & 3 \\
\hline 416 & 40 & 1 & 1 \\
\hline 435 & 166 & 4 & 2 \\
\hline 436 & 166 & 3 & 1 \\
\hline 463 & 217 & 1 & 1 \\
\hline 478 & 166 & 4 & 1 \\
\hline 544 & 40 & 2 & 3 \\
\hline 554 & 104 & 2 & 1 \\
\hline 559 & 103 & 4 & 1 \\
\hline 601 & 103 & 1 & 0 \\
\hline 602 & 103 & 0 & 3 \\
\hline $\left.\begin{array}{l}290 \\
291\end{array}\right\}$ & -67 & 2 & 2 \\
\hline \multirow{3}{*}{\multicolumn{2}{|c|}{$\begin{array}{l}\text { Total.... } \\
\text { Most probable } \\
\text { expectation. }\end{array}$}} & 40 & 24 \\
\hline & & & \\
\hline & & 48 & 16 \\
\hline
\end{tabular}

TABLE 56.

Matings of wild hybrid females with guinea-pig males, the latter homozygous in roughness.

\begin{tabular}{|c|c|c|}
\hline \multicolumn{2}{|c|}{ Parents. } & Offspring. \\
\hline \multicolumn{2}{|c|}{ 아rf $\times \sigma^{\top} \mathrm{RfRf}$} & Rfrf \\
\hline $\begin{array}{r}\frac{1}{8} \text { wild } 127 \\
222\end{array}$ & $\begin{array}{l}2034 \\
2034\end{array}$ & $\begin{array}{l}2 \\
5\end{array}$ \\
\hline \multicolumn{2}{|c|}{ q Rirf $\times \sigma^{7} \operatorname{RfRf}$} & \\
\hline \multirow{2}{*}{$\begin{array}{r}\frac{1}{3} \frac{1}{2} \text { wild } 2430 \\
\text { Total. }\end{array}$} & 2415 & 3 \\
\hline & $\ldots \ldots$ & 10 \\
\hline
\end{tabular}

\section{TABLE 54.}

Matings of $\frac{1}{82}$ wild females with guinea-pig males, in which both parents are heterozygous in color.

\begin{tabular}{|c|c|c|c|}
\hline & & \multicolumn{2}{|c|}{ Offspring. } \\
\hline \& $\mathrm{Cc}$ & $\sigma^{7} \mathrm{Cc}$ & \multicolumn{2}{|c|}{$\mathrm{CC}$ or $\mathrm{Cc}+\mathrm{cc}$} \\
\hline $\begin{array}{l}702 \\
733 \\
745\end{array}$ & $\begin{array}{l}201 \\
201 \\
201\end{array}$ & $\begin{array}{l}1 \\
3 \\
1\end{array}$ & $\begin{array}{l}1 \\
0 \\
1\end{array}$ \\
\hline \multicolumn{2}{|c|}{$\begin{array}{l}\text { Total.... } \\
\text { Most probable } \\
\text { expectation. }\end{array}$} & $\left\{\begin{array}{l}5 \\
5 \\
6\end{array}\right.$ & $\begin{array}{l}2 \\
2 \\
1\end{array}$ \\
\hline
\end{tabular}

\section{TABLE 55 .}

Summary of Tables 51-54, in which we expect 9 colored animals to 1 albino.

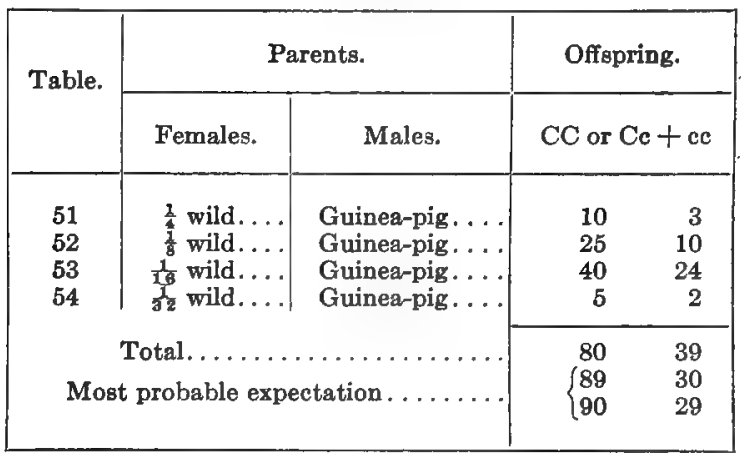

\section{TABLE 57.}

Matings of guinea-pig females, heterozygous in roughness, with smooth wild Cavia rufescens male.

\begin{tabular}{|c|c|c|c|}
\hline & & \multicolumn{2}{|c|}{ Offspring. } \\
\hline q Rfr & riffi & \multicolumn{2}{|c|}{ Rfrf $+\operatorname{rfrf}$} \\
\hline $\begin{array}{l}1125 \\
1625\end{array}$ & $\begin{array}{l}1 \\
1\end{array}$ & $\begin{array}{l}3 \\
1\end{array}$ & $\begin{array}{l}4 \\
3\end{array}$ \\
\hline \multicolumn{2}{|c|}{$\begin{array}{l}\text { Total.... } \\
\text { Most probable } \\
\text { expectation. }\end{array}$} & $\left\{\begin{array}{l}4 \\
5 \\
6\end{array}\right.$ & $\begin{array}{l}7 \\
6 \\
5\end{array}$ \\
\hline
\end{tabular}


TABLES.

TABLE 58.

Matings of wild hybrid females with guinea-pig males, in which one parent is heterozygous in roughness.

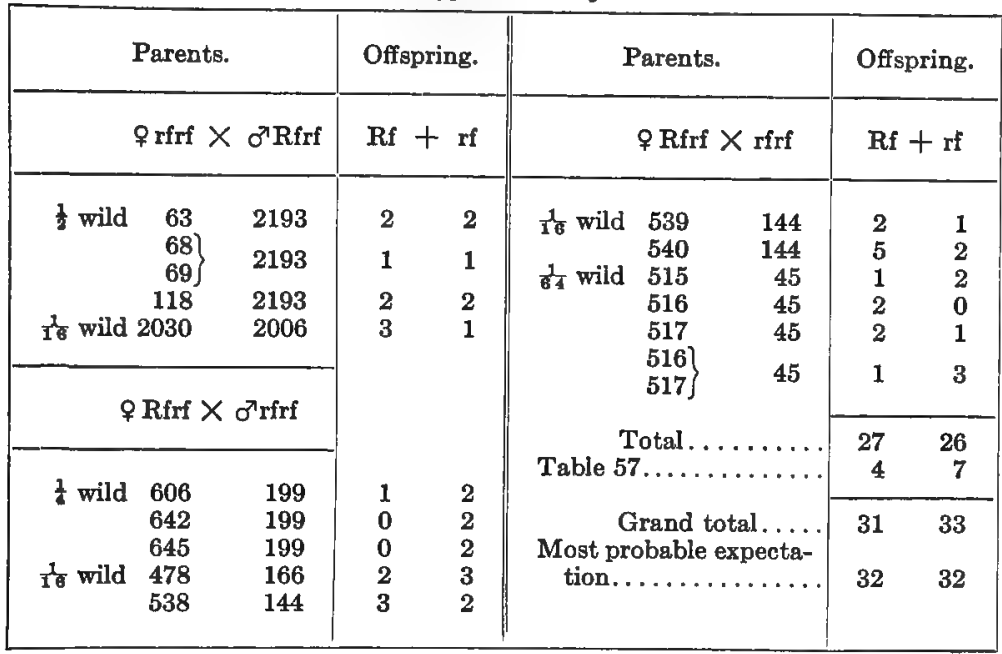

TABLE 59.

Average weights of males of the parent races and hybrids, calculated at regular intertals.

\begin{tabular}{|c|c|c|c|c|c|c|c|c|c|c|c|c|c|c|c|c|}
\hline \multirow{2}{*}{ 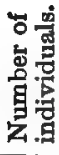 } & \multirow{2}{*}{ Class. } & \multicolumn{15}{|c|}{ Days for which the averages are calculated. } \\
\hline & & 10 & 20 & 40 & 60 & 80 & 100 & 140 & 180 & 220 & 260 & 300 & 340 & 380 & 420 & 460 \\
\hline & Wild & 95 & 128 & 206 & 265 & 313 & 341 & 378 & 393 & 400 & 407 & & 420 & 425 & & 427 \\
\hline & & 170 & 241 & 363 & 468 & 555 & 619 & 722 & 795 & 845 & 888 & 920 & 941 & 954 & 959 & 962 \\
\hline 15 & & 112 & 163 & 249 & 323 & 396 & 475 & 562 & 645 & 706 & 742 & 782 & 797 & 823 & 856 & 869 \\
\hline 62 & $\frac{1}{8}$ wil & 144 & 200 & 301 & 393 & 478 & 555 & 673 & 758 & 820 & 870 & 894 & 908 & 913 & 915 & 916 \\
\hline *28 & Guin & 130 & 184 & 283 & 369 & 437 & 494 & 603 & 677 & 724 & 768 & 799 & 812 & 816 & & \\
\hline 53 & Guinea-pi & 165 & 198 & 310 & 421 & 508 & 573 & 650 & 707 & 754 & 794 & 821 & 839 & 853 & 862 & \\
\hline
\end{tabular}

*This was a small inbred strain.

Table 60.

Average weights of females of the parent races and hybrids, calculated at regular intervals.

\begin{tabular}{|c|c|c|c|c|c|c|c|c|c|c|c|c|c|c|c|c|}
\hline \multirow{2}{*}{ 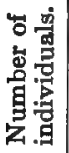 } & \multirow{2}{*}{ Class. } & \multicolumn{15}{|c|}{ Days for which the averages are calculated. } \\
\hline & & 10 & 20 & 40 & 60 & 80 & 100 & 140 & 180 & 220 & 260 & 300 & 340 & 380 & 420 & 460 \\
\hline 5 & Wild & 83 & 110 & 157 & 198 & 230 & 257 & 297 & 325 & 348 & 369 & 383 & 394 & 412 & 417 & 422 \\
\hline 9 & $\frac{1}{2}$ wild. & 178 & 235 & 331 & 412 & 480 & 530 & 601 & 656 & 703 & 736 & 766 & 788 & 823 & 828 & 832 \\
\hline 22 & wild. & 110 & 162 & 244 & 310 & 376 & 419 & 498 & 566 & 621 & 663 & 700 & 742 & 785 & 801 & 812 \\
\hline 76 & $\frac{1}{8}$ wild... & 139 & 197 & 296 & 390 & 465 & 527 & 623 & 678 & 710 & 734 & 753 & 764 & 771 & 777 & 777 \\
\hline$* 17$ & Guines-pig . . & 132 & 186 & 283 & 365 & 432 & 500 & 570 & 622 & 654 & 680 & 690 & 702 & 713 & 729 & $\ldots$ \\
\hline 56 & Guinea-pig. . & 135 & 192 & 299 & 382 & 460 & 527 & 623 & 690 & 739 & 770 & 787 & 801 & 801 & 809 & \\
\hline
\end{tabular}

*This was a small inbred strain. 
TABLE 61.

Coefficients of variability for the weights of the males in the parent races and hybrids, at six successive ages.

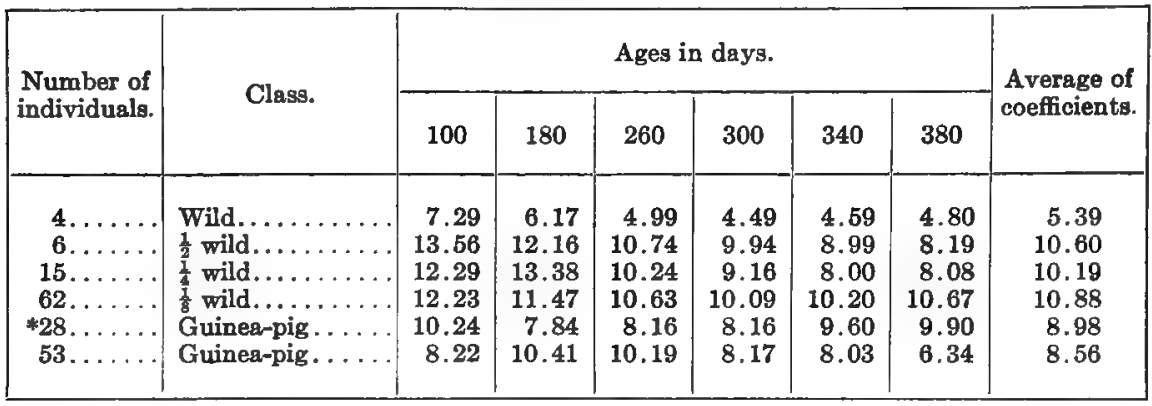

*Small inbred strain.

TABLE 62.

Coefficients of variability for the weights of the females in the parent races and hybrids, at six successive ages.

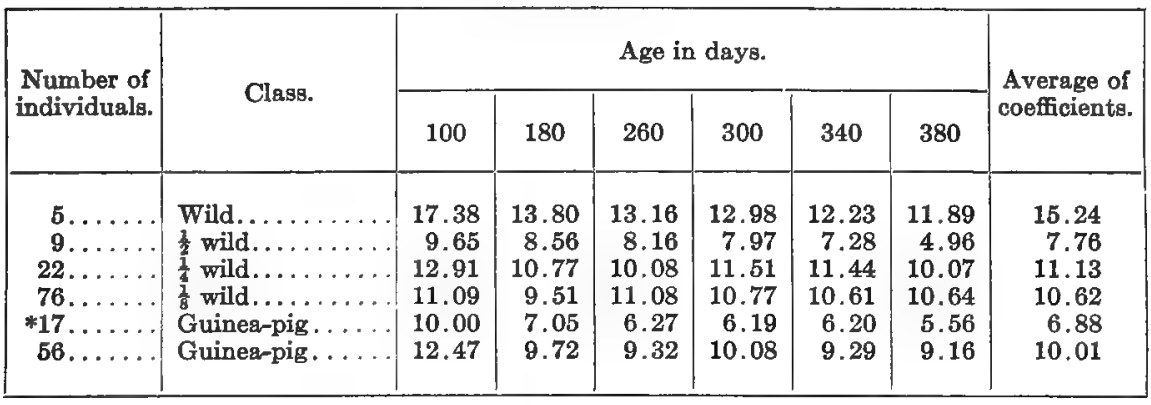

*Small inbred strain.

Table 63.

Averages in millimeters of sixteen different skeletal dimensions of the males in the parent races and in hybrids.

\begin{tabular}{|c|c|c|c|c|c|}
\hline \multirow{2}{*}{$\begin{array}{c}\text { Numbers } \\
\text { designating } \\
\text { measurements. }\end{array}$} & \multicolumn{5}{|c|}{ Number of individuals in the different classes. } \\
\hline & $\begin{array}{c}3 \\
\text { wild. }\end{array}$ & $\begin{array}{c}5 \\
\frac{1}{2} \text { wild. }\end{array}$ & $\begin{array}{l}16 \\
\text { wild. }\end{array}$ & $\begin{array}{l}60 \\
\frac{1}{8} \text { wild. }\end{array}$ & $\stackrel{78}{\text { guinea-pigs }}$ \\
\hline 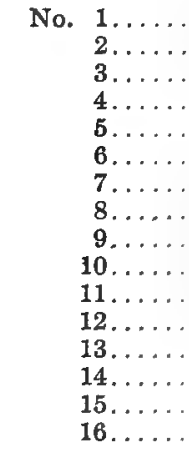 & $\begin{array}{l}60.13 \\
54.30 \\
20.03 \\
34.13 \\
27.43 \\
32.13 \\
37.13 \\
24.83 \\
34.56 \\
30.46 \\
25.26 \\
45.23 \\
24.60 \\
34.53 \\
40.53 \\
43.50\end{array}$ & $\begin{array}{l}70.58 \pm 0.38 \\
62.46 \pm 0.54 \\
22.90 \pm 0.21 \\
39.48 \pm 0.35 \\
32.48 \pm 0.21 \\
36.22 \pm 0.24 \\
42.64 \pm 0.46 \\
27.90 \pm 0.26 \\
40.40 \pm 0.31 \\
35.30 \pm 0.34 \\
31.06 \pm 0.36 \\
51.80 \pm 0.59 \\
28.16 \pm 0.33 \\
40.00 \pm 0.16 \\
44.90 \pm 0.54 \\
50.55 \pm 0.77\end{array}$ & $\begin{array}{l}66.74 \pm 0.24 \\
59.43 \pm 0.26 \\
22.20 \pm 0.15 \\
37.65 \pm 0.18 \\
29.83 \pm 0.15 \\
33.90 \pm 0.19 \\
41.01 \pm 0.18 \\
25.56 \pm 0.11 \\
38.10 \pm 0.24 \\
33.40 \pm 0.21 \\
29.22 \pm 0.14 \\
47.93 \pm 0.21 \\
27.42 \pm 0.11 \\
37.14 \pm 0.18 \\
41.28 \pm 0.20 \\
47.38 \pm 0.26\end{array}$ & $\begin{array}{l}68.98 \pm 0.17 \\
60.87 \pm 0.15 \\
22.74 \pm 0.07 \\
38.34 \pm 0.10 \\
30.80 \pm 0.09 \\
34.68 \pm 0.10 \\
41.38 \pm 0.11 \\
25.46 \pm 0.09 \\
37.72 \pm 0.12 \\
34.52 \pm 0.11 \\
29.90 \pm 0.08 \\
48.47 \pm 0.15 \\
27.86 \pm 0.08 \\
38.10 \pm 0.13 \\
42.41 \pm 0.14 \\
48.37 \pm 0.15\end{array}$ & $\begin{array}{l}68.48 \pm 0.13 \\
60.98 \pm 0.13 \\
22.33 \pm 0.06 \\
38.20 \pm 0.08 \\
30.66 \pm 0.08 \\
34.41 \pm 0.08 \\
41.48 \pm 0.08 \\
25.20 \pm 0.05 \\
37.53=0.11 \\
34.31 \pm 0.08 \\
29.67 \pm 0.07 \\
47.99 \pm 0.11 \\
27.92 \pm 0.07 \\
37.95 \pm 0.08 \\
42.06 \pm 0.10 \\
48.06 \pm 0.11\end{array}$ \\
\hline
\end{tabular}


TABLE 64.

Averages in millimeters of sixteen different skeletal dimensions of the females in the parent races and in hybrids.

\begin{tabular}{|c|c|c|c|c|c|}
\hline \multirow{2}{*}{$\begin{array}{c}\text { Numbers } \\
\text { designating } \\
\text { measurements. }\end{array}$} & \multicolumn{5}{|c|}{ Number of individuals in the different classes. } \\
\hline & $\begin{array}{c}1 \\
\text { wild. }\end{array}$ & $\begin{array}{c}8 \\
\frac{1}{2} \text { wild. }\end{array}$ & $\begin{array}{c}20 \\
\text { wild. }\end{array}$ & $\begin{array}{c}65 \\
\text { 1 wild. }\end{array}$ & $\stackrel{63}{\text { guinea-pigs }}$ \\
\hline $\begin{array}{r}\text { No. } 1 \ldots \ldots \\
2 \ldots \ldots \\
8 \ldots \ldots \\
4 \ldots \ldots \\
5 \ldots \ldots \\
6 \ldots \ldots \\
7 \ldots \ldots \\
8 \ldots \ldots \\
9 \ldots \ldots \\
10 \ldots \ldots \\
11 \ldots \ldots \\
12 \ldots \ldots \\
13 \ldots \ldots \\
14 \ldots \ldots \\
15 \ldots \ldots \\
16 \ldots \ldots \\
\end{array}$ & $\begin{array}{l}59.20 \\
52.20 \\
20.70 \\
34.20 \\
26.30 \\
30.90 \\
36.20 \\
23.80 \\
33.10 \\
28.70 \\
23.30 \\
41.60 \\
28.50 \\
31.30 \\
33.60 \\
37.50\end{array}$ & $\begin{array}{l}67.10 \pm 0.36 \\
59.41 \pm 0.33 \\
22.15 \pm 0.24 \\
38.06 \pm 0.29 \\
30.62 \pm 0.24 \\
34.21 \pm 0.19 \\
40.89 \pm 0.18 \\
27.14 \pm 0.21 \\
38.22 \pm 0.41 \\
32.12 \pm 0.22 \\
29.37 \pm 0.15 \\
50.45 \pm 0.30 \\
27.46 \pm 0.21 \\
38.44 \pm 0.36 \\
42.90 \pm 0.43 \\
48.91 \pm 0.29\end{array}$ & $\begin{array}{l}65.40 \pm 0.28 \\
57.45 \pm 0.25 \\
22.15 \pm 0.10 \\
36.96 \pm 0.17 \\
29.01 \pm 0.18 \\
32.84 \pm 0.17 \\
39.09 \pm 0.14 \\
25.23 \pm 0.14 \\
36.74 \pm 0.25 \\
31.88 \pm 0.15 \\
27.81 \pm 0.21 \\
44.46 \pm 0.25 \\
26.61 \pm 0.14 \\
36.54 \pm 0.25 \\
40.57 \pm 0.27 \\
46.13 \pm 0.31\end{array}$ & $\begin{array}{l}65.52 \pm 0.19 \\
57.41=0.16 \\
21.64 \pm 0.06 \\
36.84=0.11 \\
29.01 \pm 0.10 \\
32.37 \pm 0.10 \\
39.15 \neq 0.12 \\
24.78 \pm 0.07 \\
35.33 \pm 0.13 \\
31.88 \pm 0.08 \\
27.81 \pm 0.09 \\
46.96 \pm 0.11 \\
27.22 \pm 0.07 \\
36.88 \pm 0.11 \\
41.09 \pm 0.13 \\
46.82 \pm 0.15\end{array}$ & $\begin{array}{l}65.61 \pm 0.13 \\
58.49 \pm 0.11 \\
21.75 \pm 0.05 \\
37.28 \pm 0.08 \\
28.96 \pm 0.06 \\
32.58 \pm 0.08 \\
40.11 \pm 0.08 \\
24.81 \pm 0.06 \\
35.47 \pm 0.10 \\
32.70 \pm 0.06 \\
28.65 \pm 0.05 \\
47.67 \pm 0.11 \\
27.72 \pm 0.06 \\
37.51 \pm 0.08 \\
41.31 \pm 0.08 \\
46.89 \pm 0.10\end{array}$ \\
\hline
\end{tabular}

TABLE 65.

Standard deviations in millimeters of sixteen different skeletal dimensions of male guinea-pigs and hybrids.

\begin{tabular}{|c|c|c|c|c|}
\hline \multirow{2}{*}{$\begin{array}{c}\text { Numbers } \\
\text { designating } \\
\text { measurements. }\end{array}$} & \multicolumn{4}{|c|}{ Number of individuals in the different classes. } \\
\hline & $\begin{array}{l}5 \\
\frac{1}{2} \text { wild. }\end{array}$ & $\begin{array}{l}16 \\
\frac{1}{4} \text { wild. }\end{array}$ & $\begin{array}{l}60 \\
\frac{1}{\text { r }} \text { wild. }\end{array}$ & $\stackrel{78}{\text { guinea-pigs. }}$ \\
\hline No. $\begin{array}{r}1 \ldots \ldots \\
2 \ldots \ldots \\
3 \ldots \ldots \\
4 \ldots \ldots \\
5 \ldots \ldots \\
6 \ldots \ldots \\
7 \ldots \ldots \\
8 \ldots \ldots \\
9 \ldots \ldots \\
10 \ldots \ldots \\
11 \ldots \ldots \\
12 \ldots \ldots \\
13 \ldots \ldots \\
14 \ldots \ldots \\
15 \ldots \ldots \\
16 \ldots \ldots \\
\end{array}$ & $\begin{array}{l}1.27 \pm 0.27 \\
1.80 \pm 0.38 \\
0.68 \pm 0.15 \\
1.17 \pm 0.25 \\
0.70 \pm 0.15 \\
0.78 \pm 0.17 \\
1.53 \pm 0.33 \\
0.78 \pm 0.17 \\
1.03 \pm 0.22 \\
1.13 \pm 0.24 \\
1.18 \pm 0.25 \\
1.75 \pm 0.42 \\
1.09 \pm 0.23 \\
0.46 \pm 0.21 \\
1.59 \pm 0.38 \\
2.29 \pm 0.54\end{array}$ & $\begin{array}{l}1.40 \pm 0.17 \\
1.54 \pm 0.18 \\
0.90 \pm 0.11 \\
1.08 \pm 0.13 \\
0.87 \pm 0.10 \\
1.14 \pm 0.14 \\
1.08 \pm 0.13 \\
0.66 \pm 0.08 \\
1.40 \pm 0.17 \\
1.26 \pm 0.15 \\
0.83 \pm 0.10 \\
1.22 \pm 0.15 \\
0.66 \pm 0.08 \\
1.00 \pm 0.13 \\
1.02 \pm 0.14 \\
1.32 \pm 0.18\end{array}$ & $\begin{array}{l}1.92 \pm 0.12 \\
1.76 \pm 0.11 \\
0.75 \pm 0.05 \\
1.12 \pm 0.07 \\
1.00 \pm 0.06 \\
1.09 \pm 0.07 \\
1.27 \pm 0.08 \\
0.98 \pm 0.06 \\
1.33 \pm 0.08 \\
1.28 \pm 0.08 \\
0.96 \pm 0.06 \\
1.76 \pm 0.11 \\
0.89 \pm 0.06 \\
1.50 \pm 0.09 \\
1.57 \pm 0.10 \\
1.61 \pm 0.10\end{array}$ & $\begin{array}{l}1.74 \pm 0.09 \\
1.65 \pm 0.09 \\
0.72 \pm 0.04 \\
1.08 \pm 0.06 \\
1.00 \pm 0.05 \\
1.08 \pm 0.06 \\
1.04 \pm 0.06 \\
0.66 \pm 0.04 \\
1.40 \pm 0.08 \\
1.08 \pm 0.06 \\
0.89 \pm 0.05 \\
1.37 \pm 0.08 \\
0.84 \pm 0.05 \\
1.04 \pm 0.06 \\
1.24 \pm 0.07 \\
1.37 \pm 0.08\end{array}$ \\
\hline
\end{tabular}


TABLE 66.

Standard deviations in millimeters of sixteen different skeletal dimensions of female guinea-pigs and hybrids.

\begin{tabular}{|c|c|c|c|c|}
\hline \multirow{2}{*}{$\begin{array}{c}\text { Numbers } \\
\text { designating } \\
\text { measurements. }\end{array}$} & \multicolumn{4}{|c|}{ Number of individuals in the different classes. } \\
\hline & $\begin{array}{c}8 \\
\frac{1}{2} \text { wild. }\end{array}$ & $\begin{array}{l}20 \\
\frac{1}{4} \text { wild. }\end{array}$ & $\begin{array}{c}65 \\
\text { I wild. }\end{array}$ & $\begin{array}{c}63 \\
\text { guinea-pigs. }\end{array}$ \\
\hline No. $\begin{array}{r}1 \ldots \ldots \\
2 \ldots \ldots \\
3 \ldots \ldots \\
4 \ldots \ldots \\
5 \ldots \ldots \\
6 \ldots \ldots \\
7 \ldots \ldots \\
8 \ldots \ldots \\
9 \ldots \ldots \\
10 \ldots \ldots \\
11 \ldots \ldots \\
12 \ldots \ldots \\
13 \ldots \ldots \\
14 \ldots \ldots \\
15 \ldots \ldots \\
16 \ldots \ldots\end{array}$ & $\begin{array}{l}1.49=0.25 \\
1.38 \pm 0.23 \\
1.01 \pm 0.17 \\
1.21 \pm 0.20 \\
0.99 \pm 0.17 \\
0.79 \pm 0.13 \\
0.76 \pm 0.13 \\
0.87 \pm 0.15 \\
1.71 \pm 0.29 \\
0.94 \pm 0.16 \\
0.64=0.11 \\
1.25 \pm 0.21 \\
0.87 \pm 0.15 \\
1.50 \pm 0.25 \\
1.82 \pm 0.31 \\
1.22 \pm 0.21\end{array}$ & $\begin{array}{l}1.82 \pm 0.20 \\
1.68 \pm 0.18 \\
0.68 \pm 0.07 \\
1.14 \pm 0.12 \\
1.17 \pm 0.13 \\
1.10 \pm 0.12 \\
0.95 \pm 0.10 \\
0.91 \pm 0.10 \\
1.67 \pm 0.18 \\
0.99 \pm 0.11 \\
1.37 \pm 0.15 \\
1.59 \pm 0.17 \\
0.92 \pm 0.10 \\
1.60 \pm 0.18 \\
1.72 \pm 0.19 \\
1.98 \pm 0.22\end{array}$ & $\begin{array}{l}2.24=0.13 \\
1.88 \pm 0.11 \\
0.75 \pm 0.04 \\
1.33 \pm 0.08 \\
1.13 \pm 0.07 \\
1.13 \pm 0.07 \\
1.36 \pm 0.08 \\
0.87 \pm 0.05 \\
1.50 \pm 0.09 \\
0.95 \pm 0.06 \\
1.01 \pm 0.06 \\
1.29 \pm 0.08 \\
0.84 \pm 0.05 \\
1.33 \pm 0.08 \\
1.46 \pm 0.09 \\
1.78 \pm 0.11\end{array}$ & $\begin{array}{l}1.50 \pm 0.09 \\
1.29 \pm 0.08 \\
0.53 \pm 0.03 \\
0.96 \pm 0.06 \\
0.72 \pm 0.04 \\
0.94 \pm 0.06 \\
0.93 \pm 0.06 \\
0.70 \pm 0.04 \\
1.14 \pm 0.07 \\
0.66 \pm 0.04 \\
0.64 \pm 0.04 \\
1.22 \pm 0.08 \\
0.67 \pm 0.04 \\
0.83 \pm 0.05 \\
0.91 \pm 0.06 \\
1.09 \pm 0.07\end{array}$ \\
\hline
\end{tabular}

TABLE 67.

Coefficients of variability of sixteen different skeletal dimensions of the males in the ouinea-pigs and hybrids.

\begin{tabular}{|c|c|c|c|c|}
\hline \multirow{2}{*}{$\begin{array}{c}\text { Numbers } \\
\text { designating } \\
\text { measurements. }\end{array}$} & \multicolumn{4}{|c|}{ Number of individuals in the different classes. } \\
\hline & $\frac{5}{\frac{1}{2}}$ wild. & $\begin{array}{l}16 \\
\frac{1}{4} \text { wild. }\end{array}$ & $\begin{array}{c}60 \\
\frac{1}{8} \text { wild. }\end{array}$ & $\begin{array}{c}78 \\
\text { guinea-pigs. }\end{array}$ \\
\hline 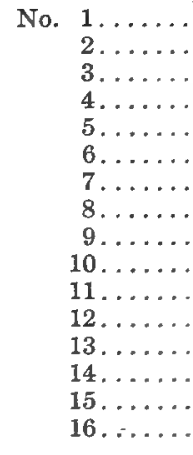 & $\begin{array}{l}1.81 \pm 0.39 \\
2.88 \pm 0.61 \\
2.99 \pm 0.64 \\
2.96 \pm 0.63 \\
2.15 \pm 0.46 \\
2.16 \pm 0.46 \\
3.59 \pm 0.77 \\
2.79=0.60 \\
2.54 \pm 0.54 \\
2.79 \pm 0.60 \\
3.79 \pm 0.81 \\
3.38 \pm 0.81 \\
3.88 \pm 0.83 \\
1.16 \pm 0.28 \\
3.55 \pm 0.85 \\
4.52 \pm 1.08\end{array}$ & $\begin{array}{l}2.09 \pm 0.25 \\
2.59 \pm 0.31 \\
4.11 \pm 0.49 \\
2.86 \pm 0.34 \\
2.93 \pm 0.35 \\
3.36 \pm 0.40 \\
2.63 \pm 0.31 \\
2.58 \pm 0.31 \\
3.67 \pm 0.44 \\
3.76 \pm 0.45 \\
2.84 \pm 0.34 \\
2.54 \pm 0.30 \\
2.41 \pm 0.29 \\
2.68 \pm 0.34 \\
2.46 \pm 0.34 \\
2.80 \pm 0.36\end{array}$ & $\begin{array}{l}2.78 \pm 0.17 \\
2.89 \pm 0.18 \\
3.29 \pm 0.20 \\
2.92 \pm 0.18 \\
3.23 \pm 0.20 \\
3.13 \pm 0.19 \\
3.06 \pm 0.19 \\
3.87 \pm 0.24 \\
3.52 \pm 0.22 \\
3.70 \pm 0.23 \\
3.21 \neq 0.20 \\
3.64 \pm 0.22 \\
3.18 \pm 0.20 \\
3.94=0.24 \\
3.70 \pm 0.23 \\
3.33 \neq 0.21\end{array}$ & $\begin{array}{l}2.54 \pm 0.14 \\
2.71 \pm 0.15 \\
3.23 \pm 0.17 \\
2.84 \pm 0.15 \\
3.25 \pm 0.17 \\
3.13 \pm 0.17 \\
2.50 \pm 0.14 \\
2.60 \pm 0.14 \\
3.73 \pm 0.20 \\
3.16 \pm 0.17 \\
2.98 \pm 0.15 \\
2.86 \pm 0.16 \\
3.00 \pm 0.17 \\
2.74 \pm 0.15 \\
2.94 \pm 0.16 \\
2.86 \pm 0.16\end{array}$ \\
\hline
\end{tabular}


TABLE 68.

Coefficients of variability of sixteen different skeletal dimensions of the females in the guinea-pigs and hybrids.

\begin{tabular}{|c|c|c|c|c|c|}
\hline \multirow{2}{*}{\multicolumn{2}{|c|}{$\begin{array}{c}\text { Numbers } \\
\text { designating } \\
\text { measurements. }\end{array}$}} & \multicolumn{4}{|c|}{ Number of individuals in the different classes. } \\
\hline & & $\begin{array}{c}8 \\
\frac{1}{2} \text { wild. }\end{array}$ & $\begin{array}{c}20 \\
\frac{1}{2} \text { wild. }\end{array}$ & $\begin{array}{c}65 \\
\frac{1}{8} \text { wild. }\end{array}$ & $\begin{array}{c}63 \\
\text { guinea-pigs. }\end{array}$ \\
\hline \multirow[t]{16}{*}{ No. } & 1 . & $2.22 \pm 0.37$ & $2.79 \pm 0.30$ & $3.41 \pm 0.19$ & $2.28 \pm 0.14$ \\
\hline & & $2.32 \pm 0.39$ & $2.92 \pm 0.31$ & $3.28 \pm 0.20$ & $2.21=0.13$ \\
\hline & 3 & $4.54 \pm 0.77$ & $3.07 \pm 0.33$ & $3.48 \pm 0.19$ & $2.46 \pm 0.15$ \\
\hline & 4 & $3.19 \pm 0.54$ & $3.09 \pm 0.33$ & $3.61 \pm 0.21$ & $2.57 \pm 0.15$ \\
\hline & 5 & $3.23 \pm 0.54$ & $4.05 \pm 0.44$ & $3.91 \pm 0.23$ & $2.47 \pm 0.15$ \\
\hline & 6 & $2.30 \pm 0.39$ & $3.35 \pm 0.37$ & $3.49 \pm 0.21$ & $2.88 \pm 0.17$ \\
\hline & 7 & $1.87 \pm 0.32$ & $2.43 \pm 0.26$ & $3.49 \pm 0.21$ & $2.32 \pm 0.14$ \\
\hline & 8. & $3.22=0.54$ & $3.63 \pm 0.39$ & $3.52 \pm 0.21$ & $2.82 \pm 0.17$ \\
\hline & 9 & $4.47=0.75$ & $4.54 \pm 0.48$ & $4.24 \pm 0.25$ & $3.23 \pm 0.19$ \\
\hline & 10 & $2.92 \pm 0.49$ & $3.11 \pm 0.35$ & $2.98 \pm 0.18$ & $2.00 \pm 0.12$ \\
\hline & 11 & $2.17 \pm 0.37$ & $4.93 \pm 0.54$ & $3.60 \pm 0.21$ & $2.24 \pm 0.13$ \\
\hline & 12 & $2.49 \pm 0.42$ & $3.57 \pm 0.39$ & $2.76 \pm 0.17$ & $2.55 \pm 0.16$ \\
\hline & 13 & $3.17 \pm 0.53$ & $3.46 \pm 0.37$ & $3.08 \pm 0.18$ & $2.41 \pm 0.15$ \\
\hline & & $3.91 \pm 0.66$ & $4.37 \pm 0.48$ & $3.61 \pm 0.22$ & $2.21 \pm 0 .-14$ \\
\hline & & $4.24 \pm 0.71$ & $4.24=0.47$ & $3.56 \pm 0.22$ & $2.19 \pm 0.14$ \\
\hline & & $2.49 \pm 0.42$ & $4.29 \pm 0.47$ & $3.81 \pm 0.23$ & $2.32 \pm 0.15$ \\
\hline
\end{tabular}

TABLE 69.

Averages of the coefficients of variability of sixteen measurements in the hybrids and guinea-pig.*

\begin{tabular}{|c|c|c|}
\hline Class. & Males. & Females. \\
\hline$\frac{1}{2}$ wild........ & $2.93 \pm 0.17$ & $3.05=0.13$ \\
$\frac{1}{4}$ wild ........ & $2.89 \pm 0.09$ & $3.62 \pm 0.10$ \\
$\frac{1}{8}$ wild........ & $3.34=0.05$ & $3.45 \pm 0.05$ \\
Guinea-pig..... & $2.94 \pm 0.04$ & $2.45 \pm 0.04$ \\
\hline
\end{tabular}

*The probable errors were calculated from the formula:

$$
E_{m}=\frac{1}{n} \sqrt{e_{1}^{2}+e_{2}^{2}+e_{3}^{2}+\ldots e_{n}^{2}}
$$

according to which the probable error of the average of a series of statistical determinations is equal to the reciprocal of the number of determinations into the square root of the sum of the squared errors of the individual determinations.

TABLE 70.

Occurrence of an inlerparietal bone.

\begin{tabular}{|c|c|c|c|c|}
\hline \multirow{2}{*}{ Class. } & \multicolumn{2}{|c|}{ With interparietal. } & \multirow{2}{*}{$\begin{array}{c}\text { Total } \\
\text { skulls } \\
\text { examined. }\end{array}$} & \multirow{2}{*}{$\begin{array}{c}\text { Percentage } \\
\text { with inter- } \\
\text { parietal. }\end{array}$} \\
\hline & Males. & Females. & & \\
\hline C. rufescens. & 0 & 0 & 7 & 0 \\
\hline C. porcellus. . & 5 & 4 & 141 & 6.4 \\
\hline$\frac{1}{2}$ wild $\ldots \ldots \ldots$ & 1 & 1 & 13 & 15.4 \\
\hline$\frac{1}{4}$ wild.... & 6 & 9 & 46 & 32.6 \\
\hline$\frac{1}{8}$ wild... & 4 & 19 & 125 & 18.4 \\
\hline
\end{tabular}


TABLE 71.

Ratios of the average of measurement 9 to the average of measurement 11; and the averages of the ratios of measurement 9 to measurement 11 in the individual skulls.

\begin{tabular}{|c|c|c|c|c|}
\hline \multirow{2}{*}{ Class. } & \multicolumn{2}{|c|}{ Ratios of averages. } & \multicolumn{2}{|c|}{ Averages of ratios. } \\
\cline { 2 - 5 } & Males. & Females. & Males. & Females. \\
\hline Wild.......... & 1.37 & 1.42 & 1.37 & 1.42 \\
$\frac{1}{2}$ wild......... & 1.30 & 1.30 & 1.29 & 1.30 \\
$\frac{1}{1}$ wild......... & 1.30 & 1.32 & 1.31 & 1.31 \\
$\frac{1}{8}$ wild......... & 1.26 & 1.26 & 1.27 & 1.27 \\
Guinea-pig.... & 1.26 & 1.24 & 1.26 & 1.24 \\
\hline
\end{tabular}

TABLE 72.

Totals of various classes of males tested by the different methods.

\begin{tabular}{|c|c|c|c|c|c|c|}
\hline Generation of male hybrids. & $\begin{array}{l}\text { Tested by } \\
\text { breeding } \\
\text { only. }\end{array}$ & $\begin{array}{c}\text { Micro- } \\
\text { scopic } \\
\text { test only. }\end{array}$ & $\begin{array}{l}\text { Tested } \\
\text { by both } \\
\text { methods. }\end{array}$ & $\begin{array}{c}\text { Total } \\
\text { breeding } \\
\text { tests. }\end{array}$ & $\begin{array}{l}\text { Total mi- } \\
\text { croscopic } \\
\text { tests. }\end{array}$ & $\begin{array}{c}\text { Total } \\
\text { number } \\
\text { tested. }\end{array}$ \\
\hline $\mathbf{F}_{1}, \frac{1}{2}$ wild $\ldots \ldots \ldots \ldots \ldots$ & 5 & 0 & 1 & 6 & 1 & 6 \\
\hline$F_{2}, \frac{1}{1}$ wild $\ldots \ldots \ldots \ldots \ldots$ & 14 & 7 & 1 & 15 & 8 & 22 \\
\hline$F_{3}, \frac{1}{8}$ wild $\ldots \ldots \ldots \ldots \ldots$ & 25 & 28 & 21 & 46 & 49 & 74 \\
\hline$F_{4}, \frac{1}{16}$ wild $\ldots \ldots \ldots \ldots \ldots$ & 6 & 55 & 44 & 50 & 99 & 105 \\
\hline$F_{6}, a_{2}^{1}$ wild $\ldots \ldots \ldots \ldots$ & 0 & 123 & 27 & 27 & 150 & 150 \\
\hline$F_{6}, \frac{1}{64}$ wild $\ldots \ldots \ldots \ldots$ & 0 & 45 & 4 & 4 & 49 & 49 \\
\hline $\mathrm{F}_{7}, \mathrm{I}_{\mathbf{2}} \mathrm{s}$ wild $\ldots \ldots \ldots \ldots$ & 0 & 14 & 1 & 1 & 15 & 15 \\
\hline$F_{8}, \frac{1}{56}$ wild $\ldots \ldots \ldots \ldots \ldots$ & 0 & 1 & 0 & 0 & 1 & 1 \\
\hline $\begin{array}{l}\text { Offspring of hybrid males and } \\
\text { females................. }\end{array}$ & 0 & 36 & 3 & 3 & 39 & 39 \\
\hline $\begin{array}{l}\text { Offspring of hybrid males and } \\
\text { guinea-pigs............. }\end{array}$ & 0 & 22 & 0 & 0 & 22 & 22 \\
\hline Totals. . & 50 & 331 & 102 & 152 & 433 & 483 \\
\hline
\end{tabular}

TABLE 73.

Results of a simple breeding test alone.

\begin{tabular}{|r|r|c|c|}
\hline $\begin{array}{c}\text { Generation of male } \\
\text { hybrids. }\end{array}$ & $\begin{array}{c}\text { Tested by } \\
\text { breeding } \\
\text { only. }\end{array}$ & Sterile. & Fertile. \\
\hline$F_{1}, \frac{1}{2}$ wild....... & 5 & 5 & 0 \\
$F_{2}, \frac{1}{4}$ wild....... & 14 & 14 & 0 \\
$F_{3}, \frac{1}{8}$ wild....... & 25 & 25 & 0 \\
$F_{4}, \frac{1}{16}$ wild...... & 6 & 5 & 1 \\
& 50 & 49 & 1 \\
\hline
\end{tabular}


TABLE 74.

Results of a simple microscopic test alone.

\begin{tabular}{|c|c|c|c|c|c|c|c|c|}
\hline $\begin{array}{l}\text { Generation of } \\
\text { male hybrids. }\end{array}$ & $\begin{array}{l}\text { Totals } \\
\text { having } \\
\text { only a } \\
\text { micro- } \\
\text { scopic } \\
\text { test. }\end{array}$ & $\begin{array}{l}\text { Number } \\
\text { with no } \\
\text { sperma- } \\
\text { tozoa. }\end{array}$ & $\begin{array}{c}\text { Number } \\
\text { with any } \\
\text { sperma- } \\
\text { tozoa. }\end{array}$ & $\begin{array}{l}\text { Percent- } \\
\text { age with } \\
\text { any } \\
\text { sperma- } \\
\text { tozoa. }\end{array}$ & $\begin{array}{c}\text { Number } \\
\text { with any } \\
\text { motile } \\
\text { sperma- } \\
\text { tozoa. }\end{array}$ & $\begin{array}{l}\text { Percent- } \\
\text { age with } \\
\text { any } \\
\text { motile } \\
\text { sperma- } \\
\text { tozoa. }\end{array}$ & $\begin{array}{c}\text { Number } \\
\text { with } \\
\text { many } \\
\text { motile } \\
\text { sperma- } \\
\text { tozoa. }\end{array}$ & $\begin{array}{l}\text { Percent- } \\
\text { age with } \\
\text { many } \\
\text { motile } \\
\text { sperma- } \\
\text { tozoa. }\end{array}$ \\
\hline $\mathrm{F}_{2}, \frac{1}{4}$ wild $\ldots$. & 7 & 5 & 2 & 28.6 & 0 & 00.0 & 0 & 00.0 \\
\hline$F_{3}, \frac{1}{6}$ wild $\ldots$ & 28 & 18 & 10 & 35.7 & 0 & 00.0 & 0 & 00.0 \\
\hline$F_{6}, \frac{1}{16}$ wild.. & 55 & 20 & 35 & 63.6 & 16 & 29.1 & 11 & 20.0 \\
\hline$F_{b}, \frac{1}{3}$ wild..$\ldots$ & 123 & 16 & 107 & 87.0 & 77 & 62.6 & 67 & 54.5 \\
\hline$F_{6}, \frac{1}{84}$ wild $\ldots \ldots$ & 45 & 2 & 43 & 95.6 & 33 & 73.3 & 31 & 68.9 \\
\hline$F_{7}$, I $\frac{1}{2} \overline{8}$ wild $\ldots .$. & 14 & 2 & 12 & 85.7 & 12 & 85.7 & 10 & 71.4 \\
\hline $\mathrm{F}_{8} . \frac{1}{56}$ wild $\ldots .$. & 1 & 0 & 1 & 100.0 & 1 & 100.00 & 1 & 100.0 \\
\hline $\begin{array}{l}\text { Offispring of hy- } \\
\text { brid males and } \\
\text { females......... }\end{array}$ & 36 & 3 & 33 & 91.7 & 22 & 61.1 & 18 & 50.0 \\
\hline $\begin{array}{l}\text { Offspring of hy- } \\
\text { brid males and } \\
\text { guinea-pigs.... }\end{array}$ & 22 & $\mathbf{0}$ & 22 & 100.0 & 22 & 100.0 & 21 & 95.5 \\
\hline
\end{tabular}

TABLE 75.

Results of all microscopic tests.

\begin{tabular}{|c|c|c|c|c|c|c|c|c|c|}
\hline $\begin{array}{l}\text { Generation of } \\
\text { male hybrids. }\end{array}$ & 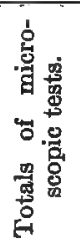 & 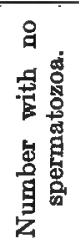 & 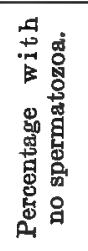 & 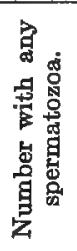 & 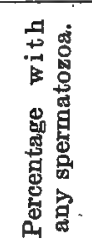 & 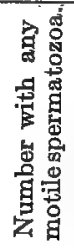 & 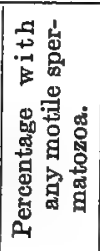 & 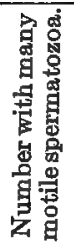 & 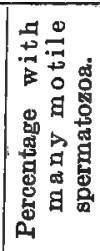 \\
\hline $\mathbf{F}_{1}, \frac{1}{2}$ wild $\ldots \ldots$ & 1 & 1 & 100.0 & 0 & 00.0 & 0 & 00.0 & 0 & 00.0 \\
\hline$F_{2}, \frac{1}{4}$ wild $\ldots .$. & 8 & 6 & 75.0 & 2 & 25.0 & 0 & 00.0 & 0 & 00.0 \\
\hline$F_{3}, \frac{1}{8}$ wild $\ldots$. & 49 & 27 & 55.1 & 22 & 44.9 & 8 & 16.3 & 7 & 14.3 \\
\hline$F_{4}, \frac{1}{16}$ wild.... & 99 & 30 & 30.3 & 69 & 69.7 & 46 & 46.5 & 33 & 33.3 \\
\hline$F_{5}$, 起 wild $\ldots \ldots$ & 150 & 18 & 12.0 & 132 & 88.0 & 102 & 68.0 & 91 & 60.7 \\
\hline$F_{6}, \frac{1}{64}$ wild $\ldots \ldots$ & 49 & 2 & 4.1 & 47 & 95.9 & 36 & 73.5 & 34 & 69.4 \\
\hline $\mathrm{F}_{7}, \frac{1}{2} \overline{8}$, wild..... & 15 & 2 & 13.3 & 13 & 86.7 & 13 & 86.7 & 11 & 73.3 \\
\hline $\mathrm{F}_{8}, \frac{1}{5} \overline{\mathrm{s}}$ wild....... & 1 & 0 & 00.0 & 1 & 100.0 & 1 & 100.0 & 1 & 100.0 \\
\hline $\begin{array}{c}\text { Offepring of hy- } \\
\text { brid males and } \\
\text { females......... }\end{array}$ & 39 & 3 & 7.7 & 36 & 92.3 & 23 & 59.0 & 19 & 48.7 \\
\hline $\begin{array}{l}\text { Offspring of hy- } \\
\text { brid males and } \\
\text { guinea-pigs.... }\end{array}$ & 22 & 0 & 00.0 & 22 & 100.0 & 22 & 100.0 & 21 & 95.5 \\
\hline Totals.. & 433 & 89 & 20.6 & 344 & 79.5 & 251 & 58.0 & 217 & 50.1 \\
\hline
\end{tabular}


TABLE 76.

Results in the combined microscopic and breeding tests.

\begin{tabular}{|c|c|c|c|c|c|c|c|c|c|c|c|c|}
\hline $\begin{array}{c}\text { Generation of male } \\
\text { hybrids. }\end{array}$ & 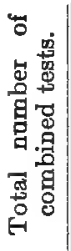 & 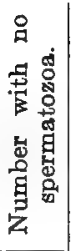 & 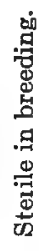 & 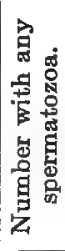 & 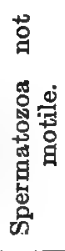 & 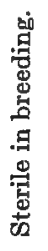 & 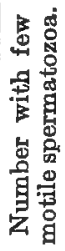 & 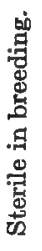 & 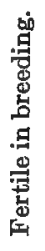 & 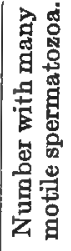 & 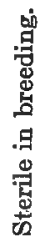 & 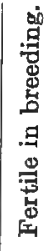 \\
\hline$F_{1}$, $\frac{1}{2}$ wild. & 1 & 1 & 1 & 0 & 0 & 0 & 0 & 0 & $\mathbf{0}$ & $\mathbf{0}$ & 0 & \\
\hline$F_{2}, \frac{1}{4}$ wild... & 1 & 1 & 1 & 0 & 0 & 0 & 0 & $\mathbf{0}$ & 0 & 0 & 0 & \\
\hline$F_{3}, \frac{3}{8}$ wild.... & 21 & 9 & 9 & 12 & 4 & 4 & 1 & 0 & 1 & 7 & 1 & 6 \\
\hline$F_{4}, \frac{x}{16}$ wild... & 44 & 10 & 10 & 34 & 4 & 4 & 8 & 8 & 0 & 22 & 6 & 16 \\
\hline$F_{5}, \frac{1}{32}$ wild. . & 27 & 2 & 2 & 25 & 0 & 0 & 1 & 1 & 0 & 24 & 6 & 18 \\
\hline$F_{6}, \frac{1}{64}$ wild... & 4 & 0 & 0 & 4 & 1 & 1 & 0 & 0 & 0 & 3 & 1 & 2 \\
\hline$F_{7}, \frac{1}{1} \frac{1}{6}$ wild...$\ldots$ & 1 & 0 & 0 & 1 & 0 & 0 & 0 & 0 & 0 & 1 & 0 & 1 \\
\hline $\begin{array}{l}\text { Offispring of hybrid males } \\
\text { and females.......... }\end{array}$ & 3 & 0 & 0 & 3 & 2 & 2 & 0 & 0 & 0 & 1 & 0 & 1 \\
\hline Totals. & 102 & 23 & 23 & 79 & 11 & 11 & 10 & 9 & 1 & 58 & 14 & 44 \\
\hline
\end{tabular}

TABLE 77.

Theoretical percentage of ultimate recessive individuals expected in back-crosses, on the basis of various numbers of factors involved.

\begin{tabular}{|c|c|c|c|c|c|c|c|c|c|}
\hline \multirow{2}{*}{ Generation. } & \multicolumn{9}{|c|}{ Number of factors. } \\
\hline & 1 & 2 & 3 & 4 & 5 & 6 & 7 & 8 & 9 \\
\hline$F_{1}, \frac{1}{2}$ wild. . & 00.00 & 00.00 & 00.00 & 00.00 & 00.00 & 00.00 & 00.00 & 00.00 & 00.00 \\
\hline $\mathbf{F}_{2}, \frac{1}{4}$ wild. . & 50.00 & 25.00 & 12.50 & 6.25 & 3.13 & 1.56 & 00.78 & 00.39 & 00.20 \\
\hline $\mathbf{F}_{3}, \frac{1}{8}$ wild $\ldots$ & 75.00 & 56.25 & 42.19 & 31.64 & 23.73 & 17.80 & 13.35 & 10.01 & 7.51 \\
\hline$F_{4}, \frac{1}{16}$ wild.... & 87.50 & 76.56 & 66.99 & 58.62 & 51.29 & 44.87 & 39.27 & 34.36 & 30.07 \\
\hline$F_{5}, \frac{1}{32}$ wild. ... & 93.75 & 87.89 & 82.40 & 77.25 & 72.42 & 67.89 & 63.65 & 59.67 & 55.94 \\
\hline$F_{6}, \frac{1}{6}$ wild .... & 96.88 & 93.85 & 90.92 & 88.07 & 85.32 & 82.66 & 80.07 & 77.57 & 75.15 \\
\hline$F_{7}$, I $\frac{1}{2} \overline{8}$ wild... & 98.44 & 96.90 & 95.39 & 93.90 & 92.43 & 90.98 & 89.56 & 88.16 & 86.79 \\
\hline$F_{8}, \frac{1}{2} \frac{1}{6}$ wild & 99.22 & 98.44 & 97.67 & 96.91 & 96.15 & 95.40 & 94.66 & 93.92 & 93.19 \\
\hline 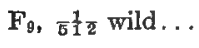 & 99.61 & 99.22 & 98.83 & 98.45 & 98.06 & 97.68 & 97.30 & 96.92 & 96.54 \\
\hline
\end{tabular}


TABLES.

TABLE 78.

Different combinations of matings which have been made.

\begin{tabular}{|c|c|c|c|c|c|c|c|c|}
\hline \multirow{2}{*}{ Males. } & \multicolumn{8}{|c|}{ Females. } \\
\hline & $\frac{1}{2}$ wild. & $\frac{1}{4}$ wild. & $\frac{1}{8}$ wild. & $\frac{1}{16}$ wild. & $\frac{1}{32}$ wild. & $\frac{1}{64}$ wild. & is 8 wild. & $\begin{array}{l}\text { Guinea- } \\
\text { pig. }\end{array}$ \\
\hline$\frac{1}{8}$ wild.... & & & $x$ & $x$ & & & & $x$ \\
\hline$\frac{1}{16}$ wild.... & & & $x$ & $x$ & $x$ & & & $x$ \\
\hline$\frac{x}{32}$ wild.... & $x$ & & & $x$ & $x$ & $x$ & & $x$ \\
\hline $6_{64}^{1}$ wild... & 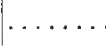 & $x$ & & & $x$ & $x$ & $x$ & $x$ \\
\hline $\mathrm{I}^{\frac{1}{2}} \overline{8}$ wild. & & & & & 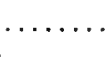 & & $x$ & $\ldots \ldots \ldots$ \\
\hline Guinea-pig. . & $x$ & $x$ & $x$ & $x$ & $x$ & $x$ & $x$ & $x$ \\
\hline
\end{tabular}

TABLE 79.

Percentages of hybrid male offspring with many motile sperm in matings of female hybrids with guinea-pigs, and female hybrids with male hybrids.

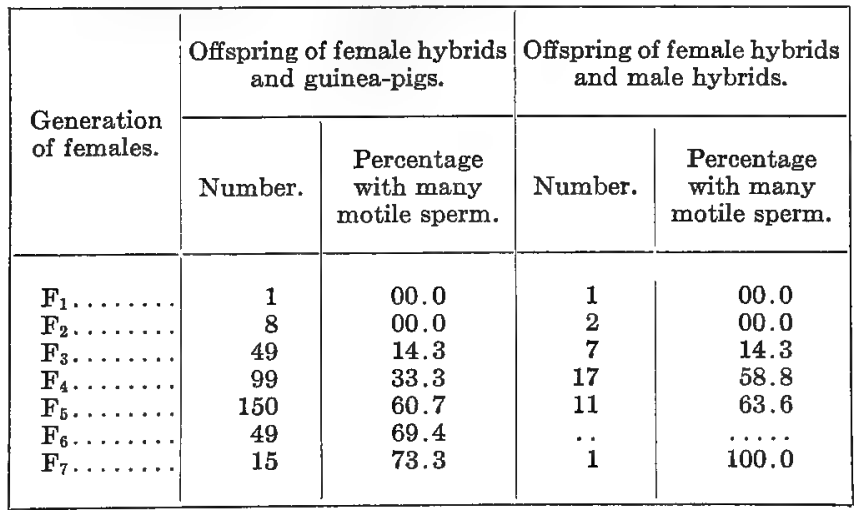

TABLE 80.

Average number of young per litter in the wild and tame parents and in the hybrids.

\begin{tabular}{|c|c|c|c|}
\hline Generation. & $\begin{array}{c}\text { Total } \\
\text { individuals. }\end{array}$ & $\begin{array}{c}\text { Number of } \\
\text { litters. }\end{array}$ & $\begin{array}{l}\text { Average } \\
\text { per litter. }\end{array}$ \\
\hline wild............... & 46 & 34 & 1.35 \\
\hline Tame $\ldots \ldots \ldots \ldots \ldots \ldots$ & 484 & 207 & 2.34 \\
\hline Tame (Minot's results)........ & 366 & 143 & 2.56 \\
\hline Tame females by wild males... & 37 & 16 & 2.31 \\
\hline $\mathbf{F}_{1}, \frac{1}{2}$ wild $\ldots \ldots \ldots \ldots \ldots$ & 83 & 52 & 1.60 \\
\hline $\mathbf{F}_{2}, \frac{1}{4}$ wild $\ldots \ldots \ldots$ & 217 & 114 & 1.90 \\
\hline$F_{3}, \frac{1}{8}$ wild $\ldots \ldots \ldots \ldots$ & 312 & 152 & 2.05 \\
\hline$F_{4}, \frac{1}{16}$ wild $\ldots \ldots \ldots \ldots \ldots$ & 344 & 172 & 1.98 \\
\hline$F_{5}, \frac{1}{32}$ wild $\ldots \ldots \ldots \ldots \ldots$ & 122 & 60 & 2.03 \\
\hline$F_{6}, \frac{1}{64}$ wild $\ldots \ldots \ldots \ldots \ldots \ldots$ & 36 & 19 & 1.89 \\
\hline
\end{tabular}


Table 81.

Ratios of sexes in the hybrids.

\begin{tabular}{|c|c|c|c|c|}
\hline Generation. & Males. & Females. & Total. & $\begin{array}{l}\text { Number of } \\
\text { males to } \\
100 \text { females. }\end{array}$ \\
\hline $\mathbf{F}_{1}, \frac{1}{2}$ wild $\ldots \ldots$ & 14 & 23 & 37 & 60.87 \\
\hline $\mathrm{F}_{2}, \frac{1}{4}$ wild $\ldots \ldots$ & 31 & 52 & 83 & 59.62 \\
\hline $\mathrm{F}_{\mathrm{s}}, \frac{1}{8}$ wild $\ldots \ldots$ & 101 & 116 & 217 & 87.07 \\
\hline$F_{4}, \frac{1}{16}$ wild $\ldots$. & 159 & 153 & 312 & 103.92 \\
\hline$F_{5}, \frac{1}{32}$ wild. . . . & 173 & 171 & 344 & 101.17 \\
\hline$F_{6}$, 妾 wild $\ldots .$. & 58 & 64 & 122 & 90.63 \\
\hline 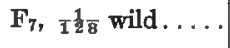 & 16 & 21 & 37 & 76.19 \\
\hline Total..... & 552 & 600 & 1152 & 92.00 \\
\hline
\end{tabular}




\section{BIBLIOGRAPHY.}

AckermanN, A.

1897. Tierbastarde, Zusammenstellung der bigherigen Beobachtungen. Abh. und Ber. des Ver. für Naturkunde in Kassell, II Wirbeltiere, Bd. 43, pp. 1-79.

Alezais, $H$.

1903 Etude anatomique sur le cobaye. Paris, F. Alcan. 170 pp., 58 fig.

Bateson, W.

1913. Mendel's principles of heredity. Univ. Press, Cambridge, Eng. xIv +413 pp., 38 fig., 6 pl.

BAUR, E.

1911. Einführung in die experimente!le Vererbungslehre. Berlin, Gebrüder Borntraeger. pp. iv $+293,80$ fig., 9 pl.

Boyd, M. M.

1908. A short account of an experiment in crossing the American bison with domestic cattle. Amer. Breeders' Ass'n. Annual, vol. 4, pp. 324-331, 4 fig.

1914. Crossing bison and cattle. Am. Journ. Heredity, vol. 5, No. 5, pp. 189-197, 7 fig.

Brainerd, E.

1907. The behaviour of the seedlings of certain violet hybrids. Science, n. s., vol. 25 , pp. 940-944.

Castle, W. E.

1905. Heredity of coat characters in guinea-pigs and rabbits. Carnegie Inst Wash., Pub. No. 23, 78 pp., 6 pl.

1906. The origin of a polydactylous race of guinea-pige. Carnegie Inst. Wash. Pub. No. 49, 29 pp.

1907. On a case of reversion induced by cross-breeding and its fixation. Seience, n. s., vol. 25, pp. 151-153.

1907a. Color varieties of the rabbit and of other rodents: Their origin and inheritance. Science, n. \&., vol. 26, pp. 287-291.

1908. A new color variety of the guinea-pig. Science, n. s., vol. 28, pp. 250-252.

- and Walter, Mollentx, and Cobr.

1909. Studies of inheritance in rabbite. Carnegie Inst. Wash. Pub. No. 114, 68 pp., 4 p].

1912. On the origin of an albino race of deermouse. Science, n. s., vol. 35, pp. $346-348$.

CuÉnot, L.

1903. L'hérédité de la pigmentation chez les souris. (2me Note.) Arch. de Zool. expér. et gén. (Sér 4), tome 1, Notes et Revue, pp. 33-41.

1904. L'hérédité de la pigmentation chez les souris. (3me Note.) Arch. de Zool. expér. et gén. (Sér. 4), tome 2, Notes et Revue, pp. 45-56.

1911. Les determinante de la couleur ohez les souris, étude comparative. (7e Note.) Arch. de Zool. expér. et gén., tome 8, Notes et Revue, pp. 40-61.

Darwin, C.

1876. The variation of animals and plants under domestication. New York, D. Appleton \& Co. Vol. I, XII +473 pp., 43 fig.; vol. II, $x+495$ pp.

Detleffen, J. A.

1912. The fertility of hybrids in a mammalian species cross. Am. Breeders' Mag., vol. III, No. 4, pp. 261-265. 
Drinkwater, H.

1908. An account of a bracbydactylous family. Proc. Roy. Soc. Edinburgh, vol. 28 , pp. $35-57,11$ fig.

DURHAim, F. M.

1908. A preliminary account of the inheritance of coat-colour in mice. Journ. of Genetics, vol. 1, pp. 159-178.

EAst, E. M.

1910. A Mendelian interpretation of variation that is apparently continuous. Am. Nat., vol. 44, pp. 65-82.

and R. A. Emerson.

1913. The inheritance of quantitative cbaracters in maize. Neb. Agr. Exp. Sta. Research Bull. No. 2, 120 pp., 21 fig.

and H. K. HAYes.

1911. Inheritance in maize. Conn. Agr. Exp. Sta. Bull. 167, 142 pp., 25 pl.

1912. Heterozygosis in evolution and in plant breeding. Bureau of Plant Ind. U. S. Dept. of Agr., Bull. 243, 58 pp., 8 pl.

Emerson, R. A.

1910. The inheritance of eizes and shapes in plants. Am. Nat., vol. 44, pp.739-746.

FARABEe, W. C.

1905. Inheritance of digital malformation in man. Papers of Peabody Museum, Harvard Univ., vol. 3. No. 3, pp. 69-77, pl. 23-27.

Focke, W. O.

1881. Die Ptlanzenmischlinge. Berlin, Gebrüder Borntraeger. Iv +569 pp.

GätNer, C. F.

1849. Versuche und Beobachtungen über die Bastarderzeugung im Pflanzenreiche. Stuttgart.

Geddes, A. C.

1910. Abnormal bone growth in the absence of functioning tegticles. Proc. Roy. Soc. Edinburgh, vol. 31 , pp. 100-150.

Giebel, C. G.

1855. Die Säugethiere. Leipsig, A. Abel. XII +1108 pp.

GoLDschmidt, R.

1912. Erblichkeitsstudien an Schmetterlingen I. 1. Untersuchungen über die Vererbung der sekundären Geschlectscharaktere und des Geschlechts.

GUYer, M. F. Ztschr. f. Abstammungs- und Vererbungslehre, Bd. 7, pp. 1-62, figs. A-X.

HAYES, H. K.

1909. On the sey of bybrid birds. Biol. Bull., vol. 16, No. 4, pp. 193-198.

1912. Correlation and inberitance in Nicotiana tabacum. Conn. Agr. Exp. Sta. Bull. 171, 45 pp., 5 pl.

HeRTwig, $O$.

1910. Die Radium Strahlung in ihrer Wirkung auf die Entwicklung thierischer Eier. (1 and 2) Sitsungb. Kön. Preus. Akad. der Wiss., Jahrg. 1910,

Hurst, C. C. pp. 221-233, 751-771.

1905. Experimental gtudies on heredity in rabbits. Linn. Soc. Journ., Zool., vol.

KING, H. D. 29, pp. 283-324.

1911. The sex in hybrid rats. Biol. Bull., vol. 21, No. 2, pp. 104-112.

IWANOFF, E. J.

1911. Die Fruchtbarkeit der Hybriden des Bos taurue und des Bison americanus Biol. Centralbl., Bd. 31, pp. 21-24.

LUND, P. W.

1841. Blik paa Bragiliens Dyreverden för sidste Jordomvoeltning. Kon. Danske Vid. Selsk., Afhand. 8, pp. 282-283, 1 pl. 
Marshald, F. H. A.

1910. The physiology of reproduction. London, Longmans, Green \& Co. XVIT +706 pp., 154 fig.

Minot, C. S.

1891. Senescence ond rejuvenation. Journ. of Physiol., No. 2, pp. 97-153, 3 pl.

Morgan, T. H.

1911. The influence of beredity and of environment in determining the coat colore

in mil e. Ann. N. Y. Acad. of Sci., vol. 21, pp. 87-117, 2 fig., 3 pl.

VoN Nathosids, $S$.

1912. Der Haustiergarten der Universität Halle. Hannover, M. und H. Schaper. 77 pp., 52 fig.

Nehring, A.

1889. Uber die Herkunft des Haus-Meerschweinchens. Sitzungsb. der Naturf. Gess. zu Berlin, No. 1, pp. 1-4, 4 fig.

1893. Uber Kreuzungen von Cavia aperea und Cavia cobaya. Sitzungsb. der Naturf. Gess. zu Berlin, No. 10, pp. 247-252.

1894. Kreuzungen von zahmen und wilden Meerschweinchen. Zool. Gart., vol. 35, pp. 1-6, 39-43, 74-78.

Nrllson-ehle, H.

1909. Kreuzungsuntersuchungen an Hafer und Weizen. Lunds Universitets Areskrift, N. F. Afd. 2, vol. 5, No. 2, 122 pp.

1911. Kreuzungsunte suchungen an Hafer und Weizen. Lunds Universitets Arsskrift, N. F. Afd. 2, vol. 7, No. 6, 82 pp.

Osborn, $\mathrm{H}$.

1908. The habits of insects as a factor in classification. Ann. of the Ent. Soc. of Am., vol. 1, No. 1, pp. 70-84, 1 pl.

Phildirs, J. C.

1912. Size inheritance in ducks. Journ. Exp. Zool., vol. 12, pp. 369-380.

Przibram, H.

1910. Experimental-Zoologie: 3. Phylogenese Inklusive Heredität. Leipsig und Wien, F. Deuticke. 315 pp., 24 pl.

PoLl, H.

1910. Uber Vögelmischlinge. Ber. über den V Intern. Ornithologen-Kongress, Berlin. pp. 399-468, 5 pl., 3 fig.

1911. Mischlingstudien VI: Eierstock und Ei bei fruchtbaren und unfruchtbaren Mischlingen. Arch. für mikros. Anat., Bd. 78, Abt. II, pp. 63-127, pl. v-vIII, 1 fig.

Rörig, A.

1903. Uber Säugetierbastarde. Der Zool. Garten, Bd. Xurv.

Sholl, G. H.

1910. Hybridization methods in corn breeding. Am. Breeder's Mag., vol. 1, No. 2, pp. 98-107, 1 fig.

SoLr.As, I. B. J.

1909. Inheritance of color and of supernumerary mammæ in guinea-pigs, with a note on the occurrence of a dwarf form. Reports to the Evol. Com. of the Roy. Soc., London. Report 5, pp. 51-79, 1 fig., 1 pl.

StandFuss, $M$.

1895. Handbuch der Paläarktigchen Gross-Schmetterlinge für Förscher und Sammler. Jena, G. Fischer. XII +392 pp., 8 fig., 8 pl.

TaMmes, T.

1911. Das Verhalten fluktuirend variierender Merkmale bei der Bastardierung. Extract du Recueil des Travaux botanique Néerlandais, vol. 8, Livr. 3, pp. 201-288, $3 \mathrm{pl}$. 
Thoмas, 0 .

1901. On mammals obtained by Mr. Alphonse Robert on the Rio Jordao, S. A., Minas Geraes. Ann. and Mag. Nat. Hist., London, vol. 8, No. 49, pp. 526-539.

DE VRIES, H.

1906. Species and varieties, their origin by mutation. Chicago, Open Court Publ. Co. XvIII +847 pp.

\section{W ALDOWTVON WAKL, H.}

1907. Fruchtbare Maultiere. Jahrb. f. wissensch. und prakt.T iersucht, vol. 2, pp. $\mathrm{LI}-\mathrm{LTV}, 1 \mathrm{pl}$.

WATERHOUge, G. R.

1848. A natural history of the mammalia. London, H. Baillière. Vol. II, 500 pp., 21 pl. 


\section{DESCRIPTION OF PLATES.}

Plate 1.

Fig. 1. Pure wild Cavia rufescens or 33.

2. $\frac{1}{2}$ wild hybrid (C. mufescens ơ $\times$ C. poreellus o $)$ ? .

3. $\frac{3}{4}$ wild hybrid ( $\frac{1}{2}$ wild hybrid $q \times C$. rufescens $\sigma^{7}$ ) \%.

Plate 2.

Fig. 4. Mid-dorsal hairs of $\frac{1}{2}$ wild hybrid. The agouti was received from the wild and is about the same shade as in the tame. In some cases the pure wild agouti was a trifle darker.

5. Mid-ventral hairs of $\frac{1}{2}$ wild hybrid. Its relation to the pure wild and pure tame is as in fig. 4 .

6. Mid-dorsal hairs of $0^{7} 804$ (1 1 wild hybrid), a typically modified, darkened agouti. The agouti was received from a pure wild strain. The ticking is very slight.

7. Mid-ventral hairs of $\sigma^{7} 804$. Compare fig. 6.

8. $0^{7804}, \frac{1}{34}$ wild hybrid. Compare figs. 6 and 7.

9. Ventral view. The same animal.

Plate 3. Photographs of male skulls in parent species and hybride.

Fig. 10. $\sigma^{7} 1$, the original wild male ancestor of all wild and bybrid animals in these experiments.

11. $0^{7} 86$, male guinea-pig.

12. $\sigma^{\top} 10, \frac{1}{2}$ wild bybrid.

13. $\sigma^{1} 151$, $\frac{1}{4}$ wild hybrid.

14. $0^{7} 206$, $\frac{1}{8}$ wild hybrid.

Nots.-These and all other reproductions of skulls and bones are natural size, and as near the calculated averages as possible, unless otherwise stated.

Plate 4. Photographs of female skulls in parent species and hybrids.

Fig. 15. $q 3$, the original wild female ancestor of all wild and bybrid animals in these experiments.

16. $\% 12656$, female guinea-pig.

17. $\$ 63$, $\frac{1}{2}$ wild hybrid.

18. $\$ 87$, $\frac{1}{6}$ wild hybrid.

19. 우 264, $\frac{1}{8}$ wild hybrid.

Plate 5. Photographs of lower jaw-bone in parent species and hybrids. Photographs of a wild male, a $\frac{1}{2}$ wild male, and a $\frac{1}{4}$ wild female skull.

Fig. 20. $\sigma^{1} 1$, the original wild male ancestor of all wild and bybrid animals in these experiments.

21. or24, pure wild gon of o'1.

22. $\sigma^{7} 78$, $\frac{1}{2}$ wild hybrid.

23. $0^{n} 111$, wild hybrid.

24. $0^{7} 169$, $\frac{1}{8}$ wild hybrid.

25. $\sigma^{7} 617$, male guinea-pig.

26. of 3 , the original wild female ancestor of all wild and bybrid animals in these experiments.

27. $q 22$, $\frac{1}{2}$ wild bybrid.

28. $\$ 96$, 1 wild hybrid.

29. $\$ 171$, $\frac{1}{8}$ wild hybrid.

30. $\$ 30$, female guinea-pig.

31. $\sigma^{7} 24$, pure wild son of $o^{7} 1$.

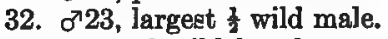

33. 우 $119, \frac{1}{4}$ wild female. 


\section{GENETIC STUDIES ON A CAVY SPECIES CROSS.}

Plate 6. Photographs of humeri and femora in the parent species and hybrids.

Fig. 34. $\sigma^{7} 1$, the original wild male ancestor of all wild and hybrid animals in these experiments.

$\sigma^{7} 24$, pure wild son of $\sigma^{1} 1$.

$\sigma^{7} 23$, $\frac{x}{2}$ wild hybrid.

$\sigma^{7} 151, \frac{1}{4}$ wild hybrid.

$\sigma^{7} 192$, $\frac{1}{3}$ wild hybrid.

o'86, guinea-pig male.

35. $\$ 3$, the original wild female ancestor of all wild and hybrid animals in these experiments.

ㅇ 22, 立 wild bybrid.

ㅇ 90 , $\frac{1}{4}$ wild bybrid.

○ 207, $\frac{1}{2}$ wild hybrid.

ㅇ 87, guinea-pig, female.

36. $\sigma^{7} 1$, the original wild male ancestor of all wild and hybrid animals in these experiments.

$\sigma^{3} 24$, pure wild son of $0^{2} 1$.

$\sigma^{7} 23$, $\frac{1}{2}$ wild hybrid.

$\sigma^{T} 151$. $\frac{1}{4}$ wild hybrid.

$\sigma^{T} 128, \frac{1}{8}$ wild hybrid.

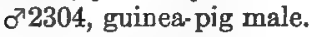

37. $\& 3$, the original wild female ancestor of all wild and hybrid animals in these experiments.

\% 75, $\frac{1}{2}$ wild bybrid.

o 87,1 wild hybrid.

\& 108 , $\frac{1}{8}$ wild hybrid.

† 12600 , guinea-pig female.

Plate 7. Photographs of scapulæ and tibiæ in the parent species and hybrids.

Fig. 38. $\sigma^{7} 24$, pure wild son of $\sigma^{7} 1$.

$0^{7} 10$, $\frac{1}{2}$ wild hybrid.

$0^{\top} 151$, $\frac{1}{4}$ wild hybrid.

$0^{7} 126$, $\frac{1}{5}$ wild hybrid.

T2034, guinea-pig male.

39 . $q 3$, the original wild female.

ㅇ $69, \frac{1}{2}$ wild hybrid.

$\$ 208$, $\frac{2}{8}$ wild hybrid.

ㅇ 87, guinea-pig female.

40. $\sigma^{\top} 1$, the original wild male.

$\sigma^{7} 24$, pure wild son of $\sigma^{7} 1$.

$\sigma^{7} 23$, $\frac{1}{2}$ wild hybrid.

o' 120 , $\frac{1}{4}$ wild hybrid.

$\sigma^{\top} 246, \frac{1}{8}$ wild hybrid.

or 12267, guinea-pig male.

41. 93 , the original wild female.

o $75, \frac{1}{2}$ wild hybrid.

o $113, \frac{1}{4}$ wild hybrid.

우 208 , $\frac{2}{8}$ wild hybrid.

○ 12601, guinea-pig female.

Plate 8. Camera-lucida drawings of the nasal-frontal sutures in the parent species and $\frac{1}{2}$ wild hybrids.

Fig. 42. Tame guinea-pig, C. porcellus.

43. Wild guinea-pig, C. rufescens.

44. $\frac{1}{2}$ wild hybrids.

Plate 9. Camera-lucida drawings of the nasal-frontal sutures in the $\frac{1}{4}$ and $\frac{1}{8}$ wild.

Fig. 45. $\frac{1}{2}$ wild.

46. $\frac{1}{8}$ wild.

Plate 10, Fig. 47. Camera-lucida drawings of the nasal-frontal sutures in the ${ }_{1 \%}^{1}$ wild hybrids. 

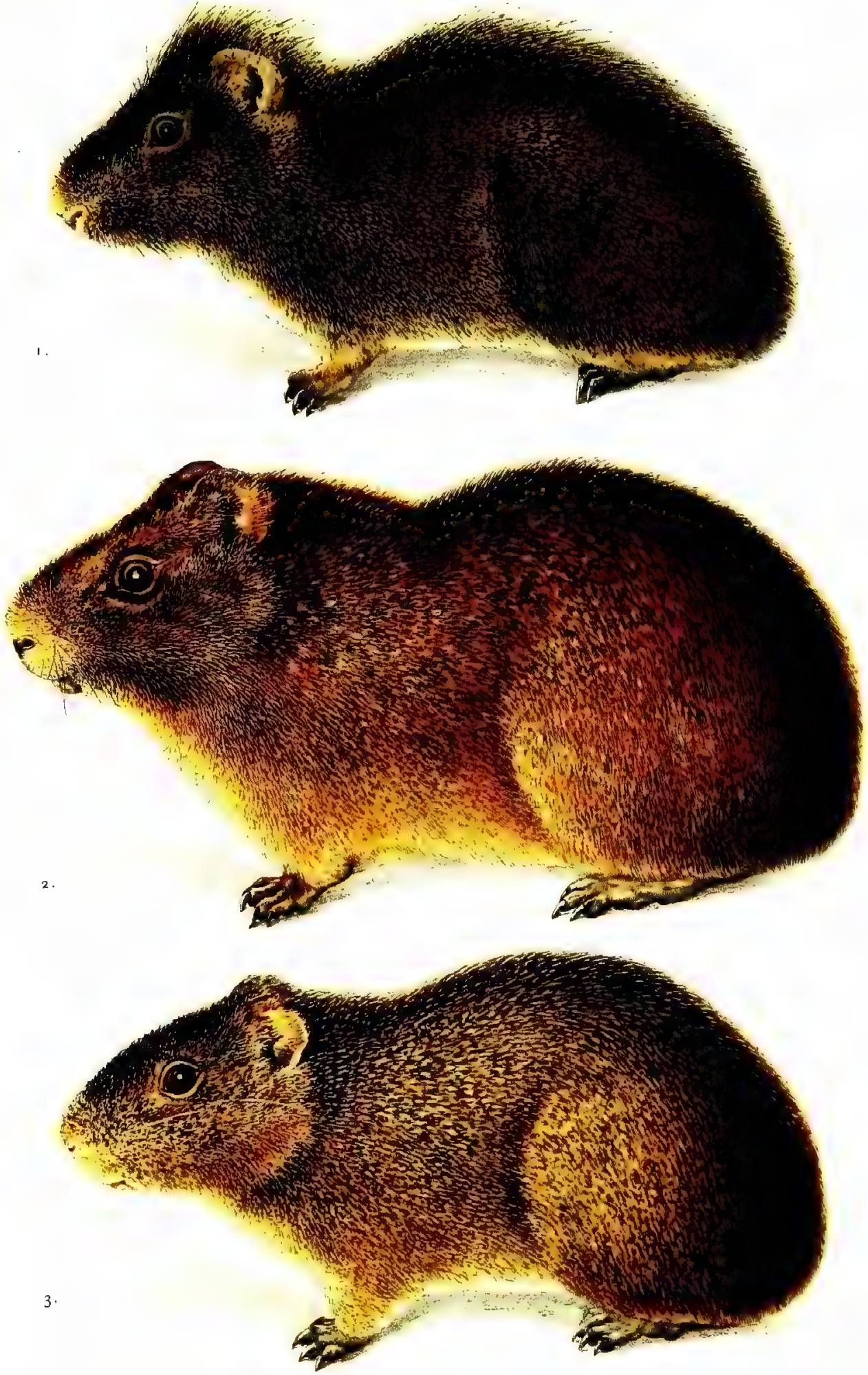

1. Pure wild Cavia rufescens $\delta 33$

2. One.half wild hybrid (C. rufescens $\sigma^{\prime} \times$ C. porcellus of) 9 .

3. Three-quarter wild hybrid ( $1 / 2$ wild hybrid $\$ \times C$, rufescens $\delta$ ) $q$ 

DETLEFSEM:
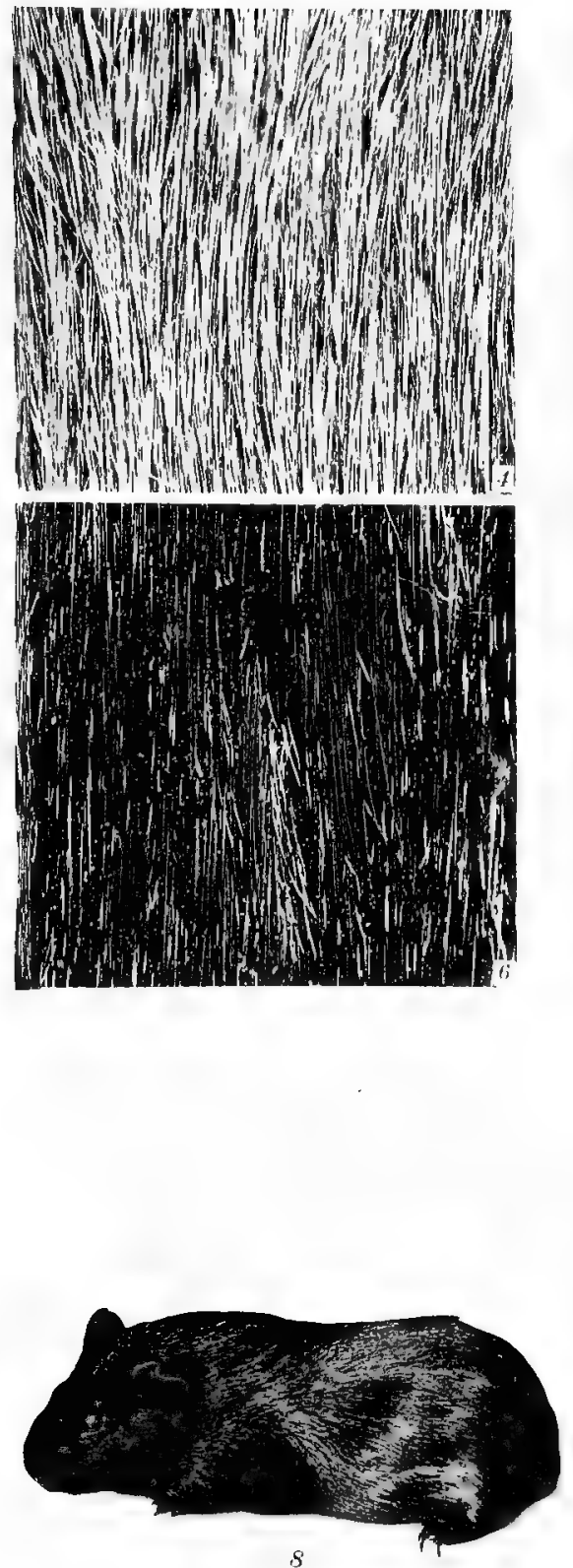

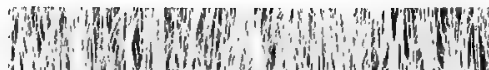

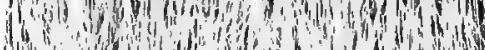

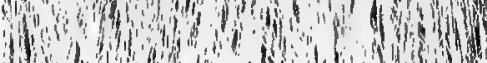
Ju low Hor on

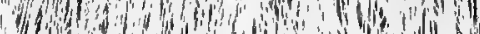
(1)

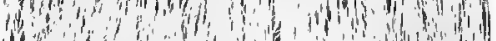
mo pos a d a

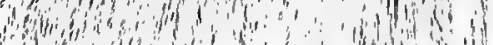
a 10 , a P)
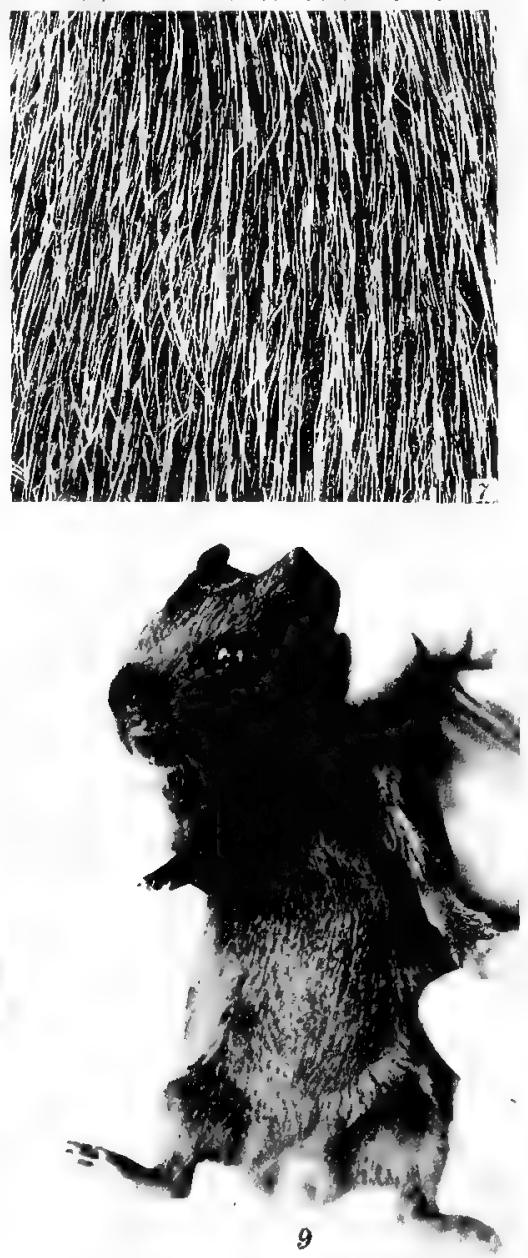

4. Mid-dorsal harrs of one-halt wilch horic.

5. Mid-rentral hairs of one-half wild hvirid,

6. Mid-dorsal hairs of distif (1/64 wild hybrid).
.

9 Viculral vicur villut anmal? 



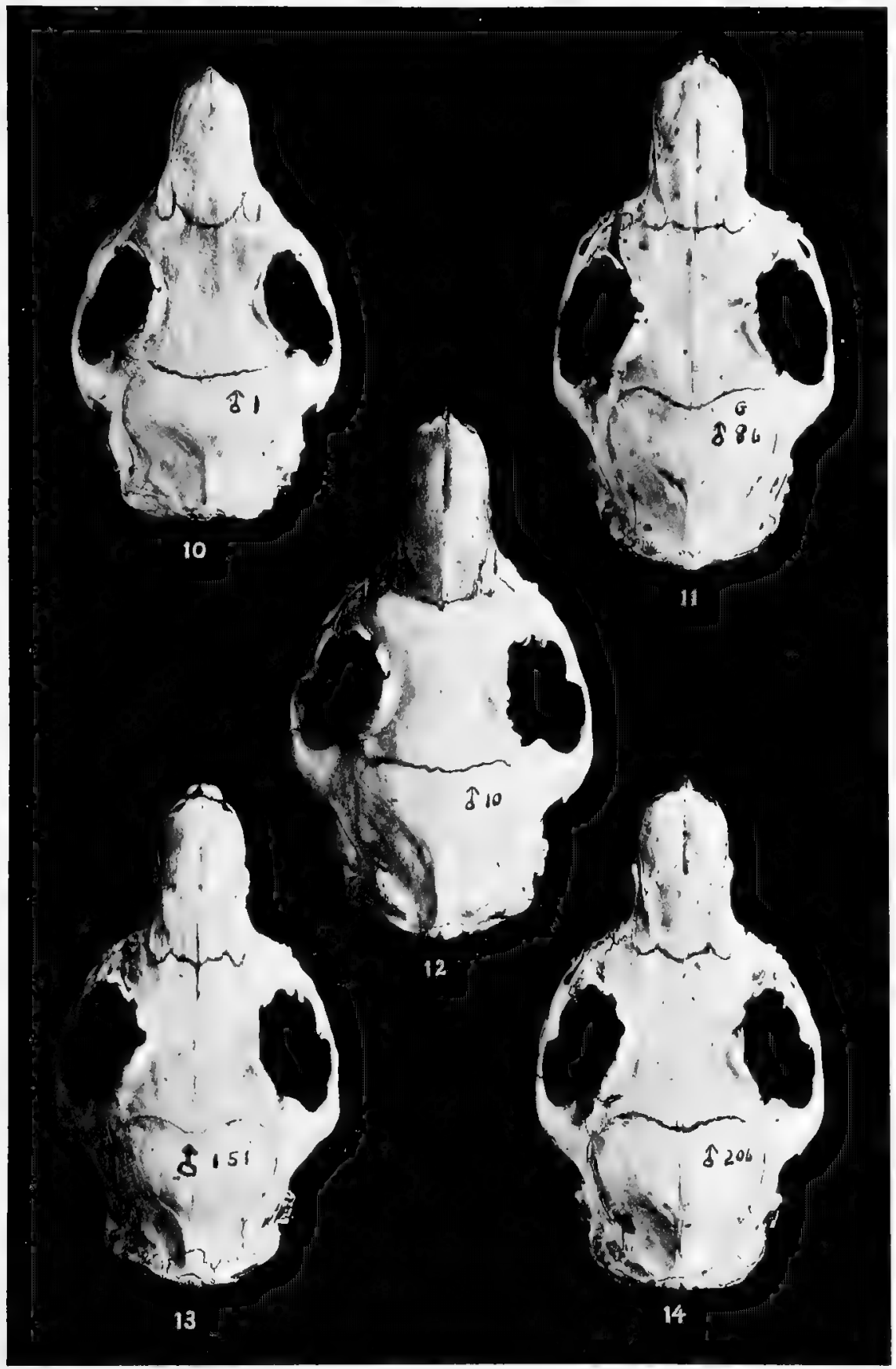

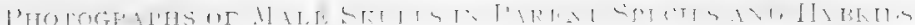

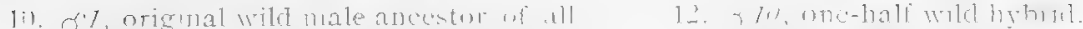

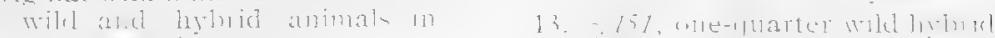

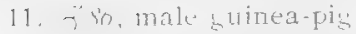




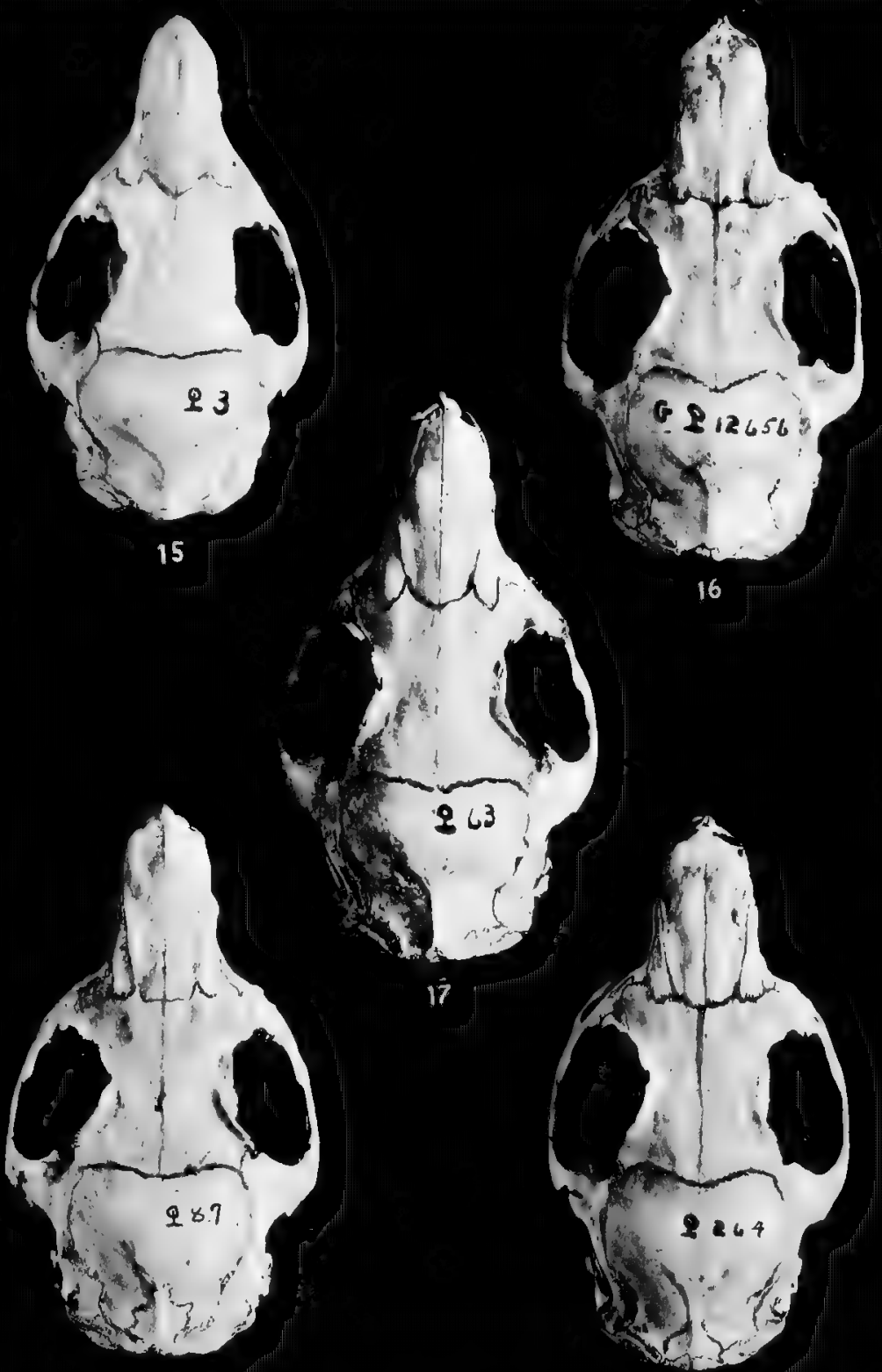

18
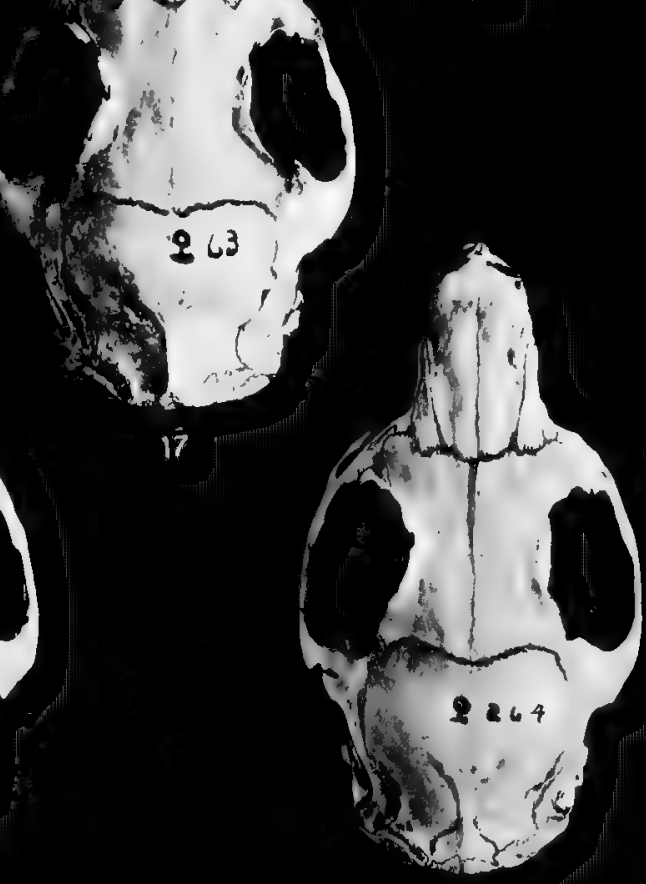

19

Photographs of Female Srulls in Parint Species and Hybrids.
15. ㅇ 3, original wild female ancestor of all
17. 우 63, one-half wild hybrid.
wild and hybrid animals in these
18. 8 87, nne-quarter wild huhrid. experiments.
19. F 261 , nne-eighth wild hvbrid.

16. 우 12656, female guinea-pig 



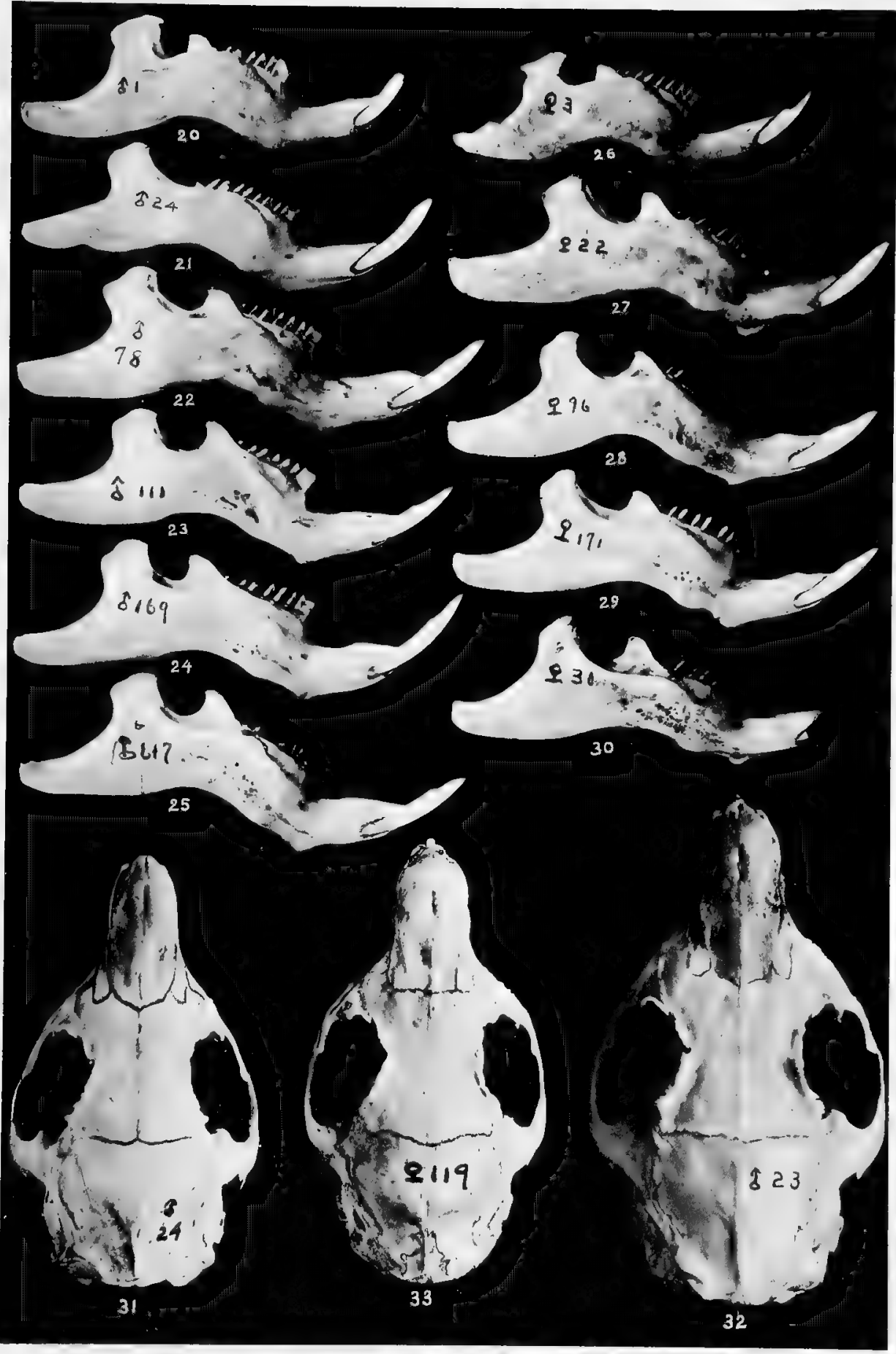

PHOTOGRAPHS OF LOWER JAW-BONE IN PARENT SPECIES AND HYBRIDS. PHOTOGRAPHS OI a Wild Male, a ONe-Half Wild Male, and a One-goarter Wild Female Skitl. 



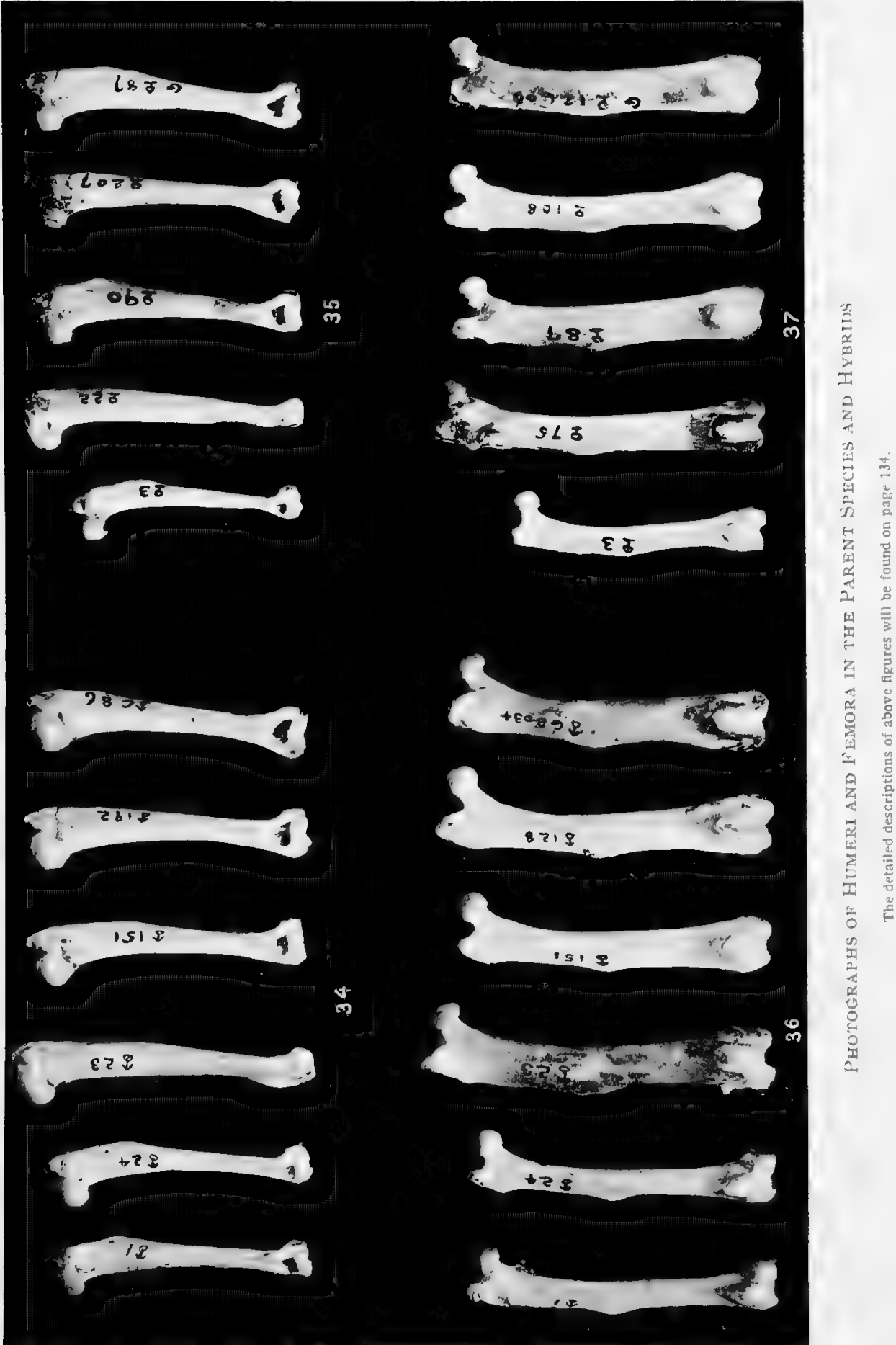





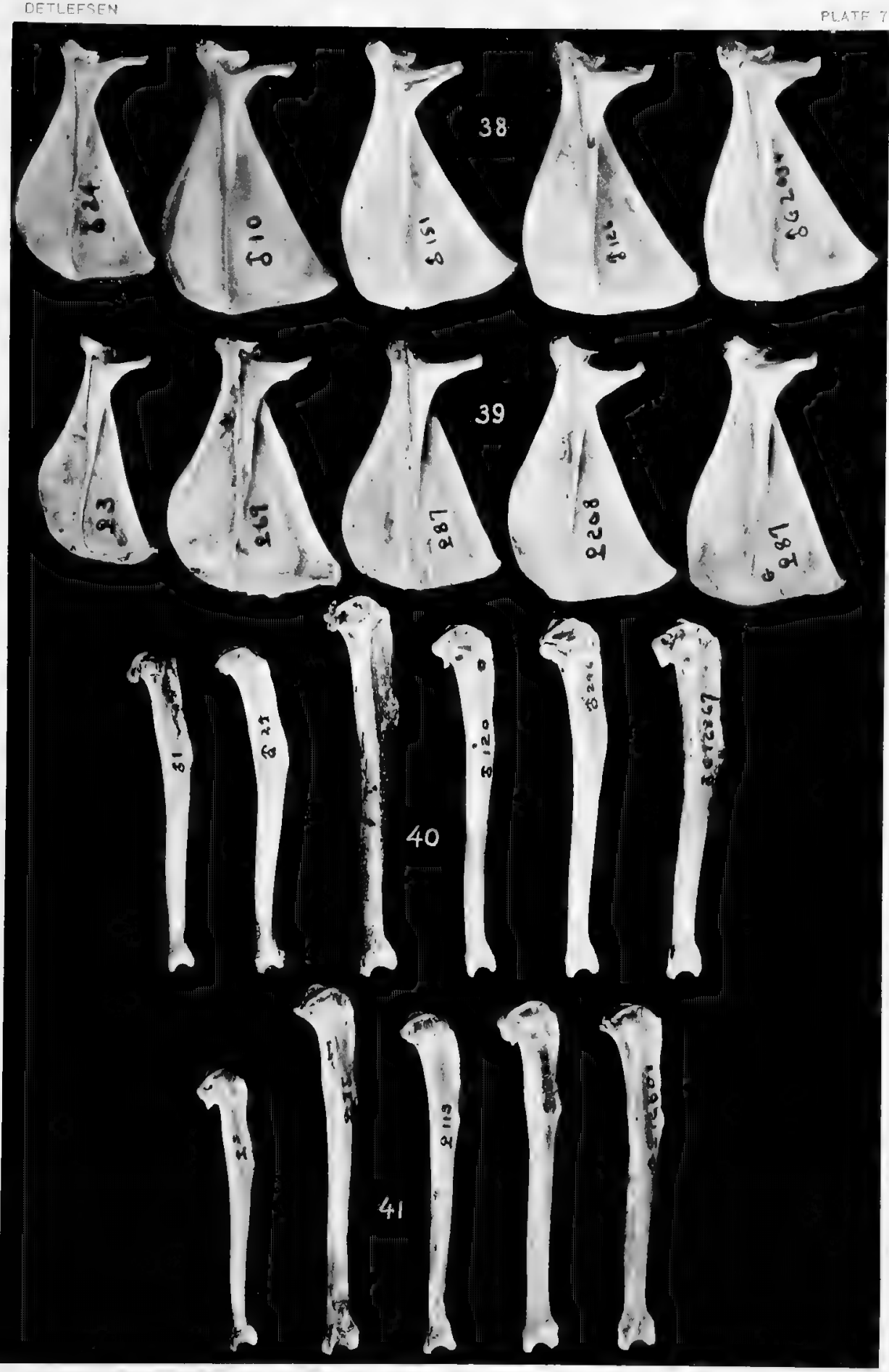

PHOTOGRAPHS OF SLAPUL.E ANI TIBI.E IN THE PARENT SPIUIES AND MYIBRIMS.

The detailed descriptions of the sbove thures will be found on page 134 . 



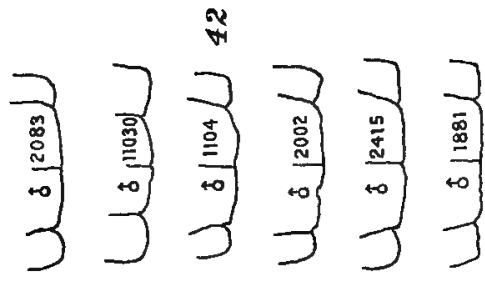

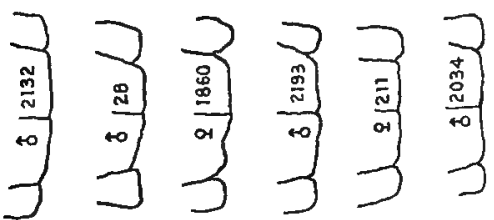

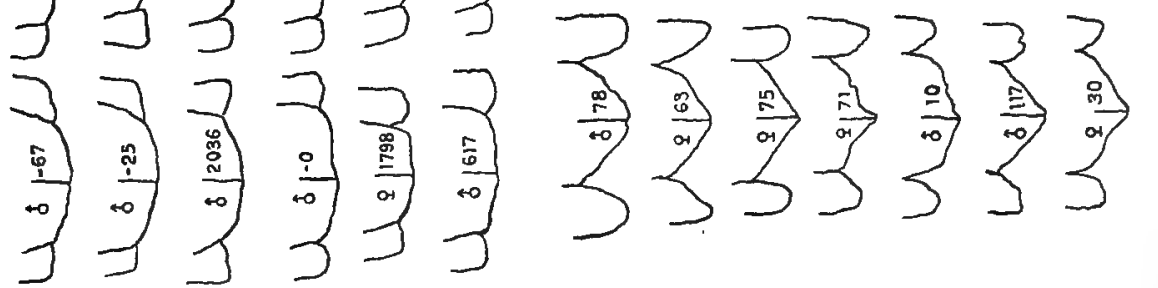

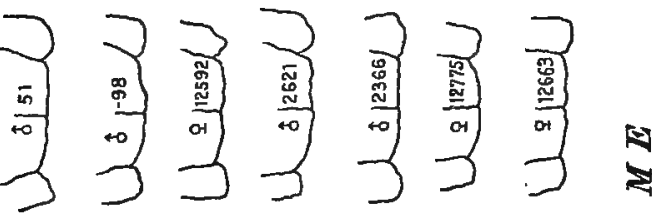

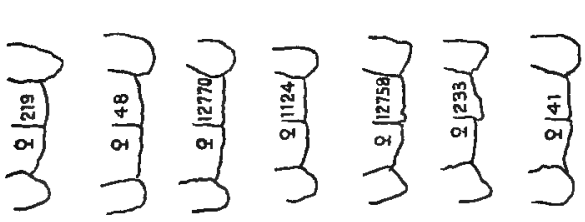

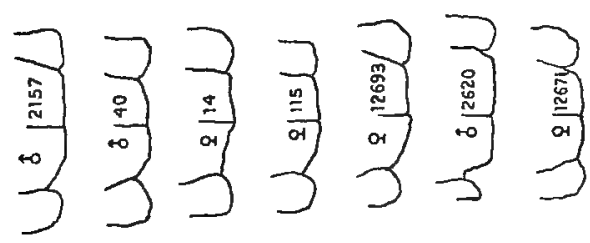

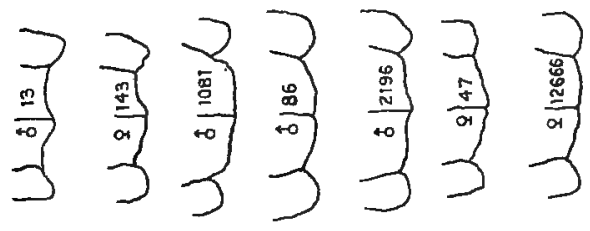

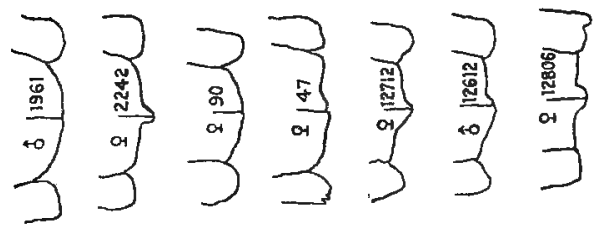

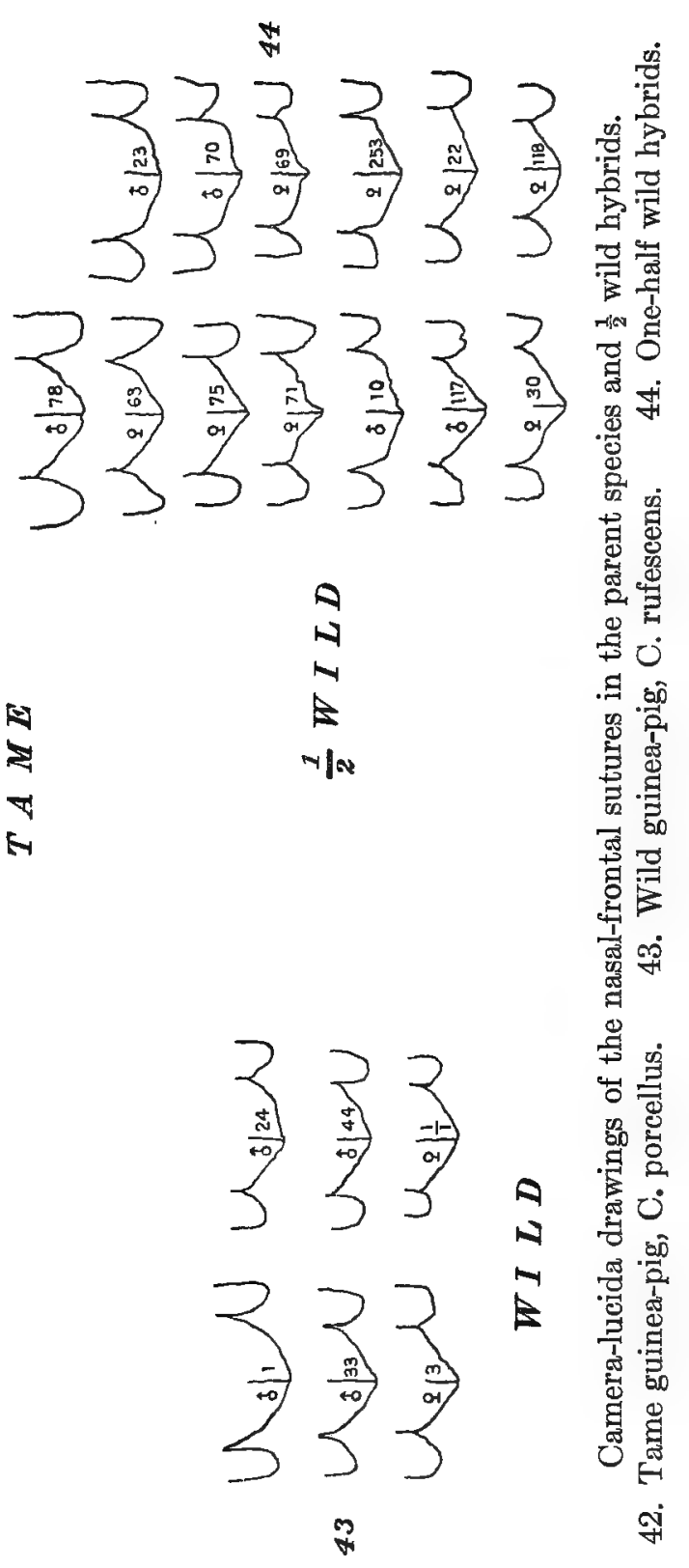






